\title{
Reibung kristalliner, amorpher und stark korrelierter Systeme unter aktiver Kontrolle
}

\section{Dissertation}

zur Erlangung des mathematischnaturwissenschaftlichen Doktorgrades

„Doctor rerum naturalium“

der Georg-August-Universität Göttingen

im Promotionsprogramm ProPhys

der Georg-August University School of Science (GAUSS)

\author{
vorgelegt von \\ Victor Manfred Pfahl \\ aus Marburg
}

Göttingen, 2018 


\section{Betreuungsausschuss}

Prof. Dr. Konrad Samwer,

I. Physikalisches Institut, Georg-August-Universität Göttingen

Prof. Cynthia A. Volkert, Ph.D.,

Institut für Materialphysik, Georg-August-Universität Göttingen

\section{Mitglieder der Prüfungskommission}

REFERENT

Prof. Dr. Konrad Samwer,

I. Physikalisches Institut, Georg-August-Universität Göttingen

\section{KORREFERENTIN}

Prof. Cynthia A. Volkert, Ph.D.,

Institut für Materialphysik, Georg-August-Universität Göttingen

\section{Weitere Mitglieder der Prüfungskommission}

Prof. Dr. Stefan Mathias,

I. Physikalisches Institut, Georg-August-Universität Göttingen

Prof. Dr. Vasily Moshnyaga,

I. Physikalisches Institut, Georg-August-Universität Göttingen

Dr. Richard Vink,

Institut für Materialphysik, Georg-August-Universität Göttingen

PD. Dr. Martin Wenderoth,

IV. Physikalisches Institut, Georg-August-Universität Göttingen

Tag der mündlichen Prüfung: 11.06.2018 
Meiner Familie 



\section{INHALTSVERZEICHNIS}

1 Einleitung 1

2 Tribologie 5

2.1 Tribologie in der Neuzeit - Historische Entwicklung . . . . . . . . . 6

2.2 Tribologie im 20. Jahrhundert - Der Weg zur Mikroskala . . . . . . 10

2.3 Moderne Tribologie - Reibung auf der Nanoskala . . . . . . . . . . 11

2.3.1 Prandtl-Tomlinson-Modell . . . . . . . . . . . . . . . 12

2.3.2 Energiedissipationsprozesse . . . . . . . . . . . . . 20

3 Methoden der Mikroskopie - Sehen, Fühlen, Hören 27

3.1 Elektronenmikroskop . . . . . . . . . . . . . . . . . . . . . . 27

3.2 Rasterkraftmikroskop . . . . . . . . . . . . . . . . . . . . . . . . . . . . . . 28

3.2.1 Kraft-Abstands-Kurve . . . . . . . . . . . . . . . . . . 31

3.2.2 Reibungsmikroskopie .................. . . . . . . . . . . . . . . 33

3.2 .3 Weitere Messmodi . . . . . . . . . . . . . . . . 34

3.3 Ultraschallmikroskopie . . . . . . . . . . . . . . . . . . . 36

3.3.1 Ultraschallkraftmikroskopie . . . . . . . . . . . . 37

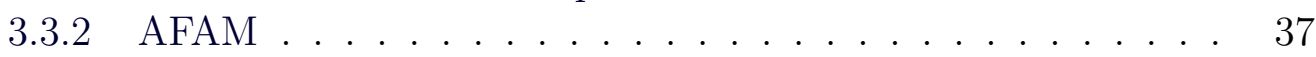

3.3.3 Kontaktresonanzmodelle . . . . . . . . . . . . . 38

4 Probensysteme 45

4.1 Manganate. . . . . . . . . . . . . . . . . . 45

4.1 Kristallstruktur . . . . . . . . . . . . . . . . 46

4.1.2 Aufhebung der Energieentartungen . . . . . . . . . . . . . . 47

4.1.3 Austauschmechanismen . . . . . . . . . . . . . . . . 48

4.1 .4 Polaronen . . . . . . . . . . . . . . . . . . 53

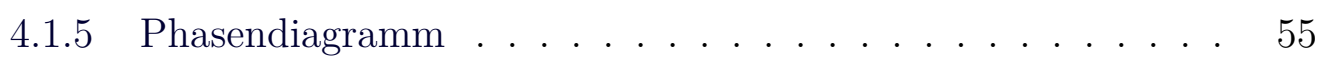

4.2 Metallische Gläser . . . . . . . . . . . . . . . . 57

4.2.1 Potentielle Energielandschaft . . . . . . . . . . . . 60

5 Probenherstellung Und -Charakterisierung 63

5.1 Metallorganische Aerosoldeposition . . . . . . . . . . . . . . 63

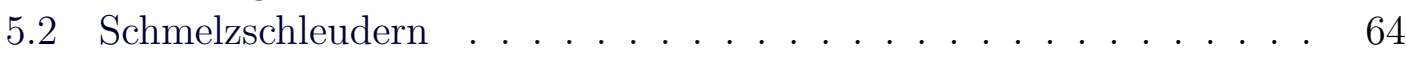

5.3 Dynamische Differenzkalorimetrie . . . . . . . . . . . . 65 
5.4 Strukturanalyse . . . . . . . . . . . . . . . . 66

5.4 .1 Röntgenbeugung . . . . . . . . . . . . . . 67

5.4 .2 Röntgenreflektometrie . . . . . . . . . . . . . . 67

5.4.3 Streuung an amorphen Systemen . . . . . . . . . . . 67

5.5 Transportmessung \& Magnetometrie . . . . . . . . . . . . . . . 68

5.6 Ergebnisse der Charakterisierung . . . . . . . . . . . . . . . 68

5.6.1 $\mathrm{La}_{0,6} \mathrm{Sr}_{0,4} \mathrm{MnO}_{3} \ldots \ldots \ldots \ldots \ldots \ldots$

5.6.2 $\mathrm{Pd}_{77,5} \mathrm{Cu}_{6} \mathrm{Si}_{16,5} \ldots \ldots \ldots \ldots \ldots$

6 Sonolubrichtion $\quad 73$

6.1 Reibungshysteresen . . . . . . . . . . . . . . . . 73

6.2 AFAM vs. UAFM . . . . . . . . . . . . . . . 76

6.3 Variation der Blattfederamplitude . . . . . . . . . . . . . 79

6.4 Material . . . . . . . . . . . . . . . . . . . . 80

6.5 Analytik . . . . . . . . . . . . . . . . . . 81

6.6 Messdaten und analytische Beschreibung . . . . . . . . . . . . . . 86

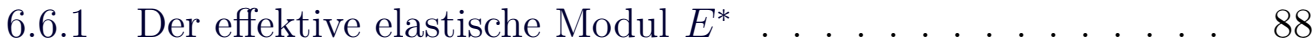

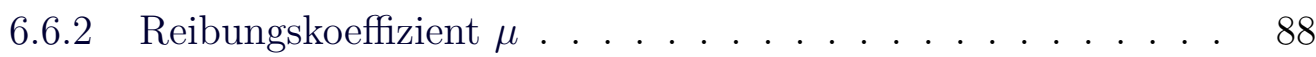

6.6.3 Spitzenradius $R \ldots \ldots$. . . . . . . . . . . 89

6.6.4 Adhäsionskonstante $\gamma \ldots \ldots$. . . . . . . . . . . 89

6.6.5 Vergleich mit Messdaten . . . . . . . . . . . . . . . . 89

6.7 Normalkraftabhängigkeit . . . . . . . . . . . . . . . . . . . . . . . . 90

6.7.1 Abweichen der Daten . . . . . . . . . . . . . . . . 92

6.8 Weitere Auftragungsmöglichkeiten . . . . . . . . . . . . . 96

7 KONTAKTRESONANZ-REIBUNGSKRAFTMIKROSKOPIE 103

7.1 Auswertung . . . . . . . . . . . . . . . . . . . . . 104

7.1.1 Resonanzkurven . . . . . . . . . . . . . . . . 104

7.1.2 Parameterberechnung . . . . . . . . . . . . 108

7.2 Ergebnisse . . . . . . . . . . . . . . . . . . . . . . 110

$7.2 .1 \mathrm{LSMO} \ldots \ldots \ldots \ldots \ldots$

7.2 .2 amorphes $\mathrm{PdCuSi}$. . . . . . . . . . . . . . . . . 112

7.2.3 kristallines $\mathrm{PdCuSi}$. . . . . . . . . . . . . . . . . 113

8 DiskUSSION 117

8.1 Allgemeines . . . . . . . . . . . . . . . . . . . 118

8.1 .1 Messspitze . . . . . . . . . . . . . . 118

8.1 .2 Wasser . . . . . . . . . . . . . . . 119

8.2 Dämpfungsprozesse . . . . . . . . . . . . . . . . . . . . . . . . . . . . . . . . . . . . 122

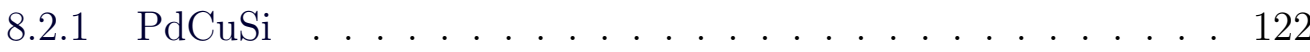

$8.2 .2 \quad \mathrm{LSMO} \ldots \ldots \ldots \ldots \ldots \ldots \ldots$ 
9 Zusammenfassung und Ausblick 139

$\begin{array}{ll}\text { LITERATURVERZEICHNIS } & 143\end{array}$

$\begin{array}{ll}\text { LEBENSLAUF } & 168\end{array}$

$\begin{array}{lr}\text { VERÖFFENTLICHUNGEN } & 169\end{array}$

$\begin{array}{lr}\text { BeITRÄGE AUF Konferenzen } & 170\end{array}$

$\begin{array}{ll}\text { DANKSAGUNG } & 171\end{array}$ 



\section{EINLEITUNG}

Während einer Relativbewegung zweier sich berührender Körper wird mechanische und kinetische Energie in Wärme umgesetzt. Dieser energiedissipative Prozess wird Reibung genannt - und er bestimmt unseren Alltag. Ohne Reibung käme nichts in Bewegung, nichts zum Halten, Kugelschreiber würden nicht funktionieren, Lampen von der Decke fallen, da Schrauben keinen Halt in der Wand fänden. Auf der anderen Seite belastet Reibung die Umwelt: Jedes Auto verbraucht ein Drittel des Treibstoffs, nur um Reibungsverluste zu überwinden [Holmberg12]. Die jeweiligen Gegebenheiten entscheiden also, ob Reibung von Vor- oder Nachteil ist.

Bis heute ist daher die grundlegende Frage der Tribologie (dt. Reibungslehre): Wie wird die Energie konvertiert? Die Antwort auf diese Frage würde erklären, über welche Kanäle die Energiedissipation stattfindet. Damit könnten Möglichkeiten gefunden werden, diese Kanäle aktiv zu beeinflussen, um so Reibung zu kontrollieren. Das ist zum einen aus wissenschaftlicher Sicht, zum anderen aber auch aus wirtschaftlicher Sicht interessant, denn bis heute ist Reibung mit etwa sechs Prozent einer der größten Verlustbeiträge des Bruttoinlandsprodukts eines Industriestaates [Persson00].

Diese Dissertation liefert einen Beitrag zum Verständnis der Reibung. Dies wird erreicht, indem Reibung auf mikroskopischer Skala studiert und über externe Stimuli aktiv beeinflusst wird. Dazu werden Materialien untersucht, die in verschiedenen Zuständen vorliegen können. Zeigen diese Zustände unterschiedliches Reibungsverhalten, kann man dies mit den zugrunde liegenden physikalischen Eigenschaften der Zustände in Zusammenhang bringen. Dadurch werden gezielt systemabhängig Dissipationskanäle untersucht.

Seit Jahrtausenden wird versucht, Reibung für die jeweilige Situation vorteilhaft zu beeinflussen. Die Erfindung des Rades - das Verwendung in Transportgeräten fand - stellt dabei einen Meilenstein dar. Im alten Ägypten sind Schmiermittel verwendet worden, um Steinstatuen einfacher zu bewegen. Es ist daher schwierig, einen historisch korrekten Zeitpunkt anzugeben, der als Ausgangspunkt der Tribologie angesehen werden kann. Leonardo da Vinci wird in der Literatur allerdings 
häufig als Erster erwähnt, wenn es um die Wissenschaft der Tribologie geht. Doch war es der Franzose Amontons, der als Erster mathematische Beschreibungen der Reibung formulierte und auch veröffentlichte, so dass bis heute in jedem Schulbuch zur Reibung von den Amontons'schen Gesetzen die Rede ist.

Bei einem Forschungsfeld mit so langer Tradition ist es notwendig, einen Überblick über die Tribologie zu geben. Da bereits in der Neuzeit empirische Gesetze formuliert worden sind, die bis heute gültig sind, wird das erste Kapitel die historische Entwicklung der Reibungslehre skizzieren.

Amontons und auch viele weitere Wissenschaftler seiner und der folgenden Zeit untersuchten Reibung auf makroskopischer Ebene. Sie konzentrierten sich vor allem auf Rauigkeiten, Adhäsionskräfte oder Schmiermittel. Bis heute wird daher Reibung häufig als eine Verzahnung der beteiligten Materialien wahrgenommen, die mit einem Schmiermittel reduziert werden kann. Die Annahme, dass Reibung nur ein materialwissenschaftliches Problem sei, greift dabei aber deutlich zu kurz. Es ist beispielsweise nicht möglich, im Vorhinein zu berechnen, wie viel Kraft benötigt wird, um ein Material gegen ein anderes zu bewegen.

Um die fundamentale Physik der Reibung genauer zu studieren, müssen daher unter anderem topographische Einflüsse ausgeschlossen werden. Erst die Entwicklung des Rasterkraftmikroskops in den Achtzigerjahren ermöglichte dies. Mit diesem Gerät konnte Reibung erstmals auch auf der atomaren Skala untersucht werden, wodurch ein neues Forschungsfeld der Tribologie eröffnet worden ist. In der sogenannten Nanotribologie gibt es im Idealfall keine Oberflächenrauigkeiten mehr, die zuvor häufig als Ursache der Reibung angesehen wurden. Als Abschluss des theoretischen Teils der Reibung werden moderne nanotribologische Arbeiten vorgestellt, die zum Verständnis der vorliegenden Arbeit beitragen, da sie ebenso Materialien untersuchen, die in unterschiedlichen Zuständen vorliegen. In diesen Arbeiten werden phononische und elektronische Dissipationsprozesse als Ursache der Reibungsänderung diskutiert.

Im Anschluss an die Reibungstheorie werden Methoden der Mikroskopie beschrieben. Dabei liegt der Fokus in der Darstellung des Rasterkraftmikroskops, das auch hier zur Reibungsmessung verwendet wird. Dieses Gerät misst die Verbiegung einer Blattfeder, an der eine Messspitze angebracht ist, die eine Probenoberfläche berührt. Dadurch ist es - neben der ursprünglichen Idee der Entwickler Gerd Binnig, Calvin F. Quate und Christoph Gerber, Oberflächen von Isolatoren abzubilden möglich, Reibungsmessungen durchzuführen. Im Reibungskraftmikroskopiemodus, der ebenso in diesem Kapitel beschrieben wird, wird die Torsion der Blattfeder 
gemessen, worüber die Reibungskraft bestimmt werden kann. Zusätzlich können externe Anregungen verwendet werden, um die Eigenfrequenzen des Systems anzuregen. Dadurch können Bereiche der Ultraschallmikroskopie auch auf atomarer Skala angewendet werden. Über geeignete Kontaktmodelle, die im dritten Kapitel beschrieben werden, können so lokale mechanische Eigenschaften der Probe gemessen werden.

Die untersuchten Proben sollen in verschiedenen Zuständen vorliegen können, damit eine eventuelle Reibungsänderung den jeweiligen Zuständen zugeordnet werden kann. Die genaue Kenntnis über die physikalischen Eigenschaften der unterschiedlichen Zustände ist daher grundlegend für die weiteren Betrachtungen. Aus diesem Grund wird im Anschluss an die Mikroskopiemethoden die Physik der untersuchten Probensysteme vorgestellt. Hierbei handelt es sich zum einen um $\operatorname{Pd}_{77,5} \mathrm{Cu}_{6} \mathrm{Si}_{16,5}$. Dieses System liegt im amorphen und kristallinen Zustand vor. Zum anderen wird das stark korrelierte $\mathrm{La}_{1-x} \mathrm{Sr}_{x} \mathrm{MnO}_{3}$ (LSMO) mit der Dotierung $x=0,4$ untersucht. LSMO zeigt einen Phasenübergang von einem ferromagnetischen zu einem paramagnetischen Metall. Dieser Phasenübergang wird hier mit der Temperatur getrieben.

Nachdem die theoretischen Eigenschaften der Systeme beschrieben sind, werden die Proben auf diese Eigenschaften hin überprüft. Bevor dies geschehen kann, müssen die Proben zunächst hergestellt werden. Dabei kommt einerseits das Schmelzschleuderverfahren zum Einsatz, um das metallische Glas $\mathrm{Pd}_{77,5} \mathrm{Cu}_{6} \mathrm{Si}_{16,5}$ herzustellen. Anderseits bietet die metallorganische Aerosoldeposition eine Möglichkeit, perfekte LSMO-Dünnfilme wachsen zu lassen. Die Proben werden anhand von Standardcharakterisierungsmethoden auf ihre Qualität überprüft. Die Funktionsweisen der verwendeten Methoden - bestehend aus Röntgen-, Magnetisierungs- ,Transportund Kalometriemessungen - werden dazu zunächst beschrieben. Dann werden die Messungen mittels ebendieser Methoden gezeigt und dadurch Struktur sowie magnetische und elektrische Eigenschaften bestimmt.

Die Informationen über die Probeneigenschaften werden im folgenden Kapitel verwendet, um Reibungsmessungen auf unterschiedlichen Zuständen durchzuführen. Dabei liegt der Fokus zunächst auf der Entwicklung einer analytischen Beschreibung der sogenannten Sonolubrication. Hierbei werden externe Anregungen im kHz-Bereich verwendet, um die verwendete Blattfeder anzuregen und so die Normalkraft zu variieren. Dadurch kann Reibung aktiv kontrolliert werden. Die analytische Beschreibung ermöglicht es außerdem, die Variation der Normalkraft in Abhängigkeit von der Anregung zu verstehen und so Sonolubrication als Messme- 
thode der Reibung zu verwenden.

Im nächsten Kapitel wird eine Messmethode verwendet, die eigentlich zur Bestimmung der internen Reibung verwendet wird. Hier jedoch wird diese Methode so verändert, dass auch Reibung auf der Oberfläche untersucht werden kann. Dadurch entsteht eine neue Messmethode der Reibung, die den Namen KontaktresonanzReibungskraftmikroskopie (CR-FFM) tragen soll. Auch hier werden externe Anregungen verwendet. Der Unterschied zur vorherigen Messmethode besteht darin, dass die Position der Messspitze nicht verändert wird, so dass diese Messung lokaler stattfindet und so beispielsweise topographische Effekte ausgeschlossen werden. Die zugrunde liegende Idee ist, die Messspitze im Kontakt derart anzuregen, dass sich ein großer Teil der Kontaktfläche auf der Probenoberfläche bewegt, ohne dass sich die Messspitze von der Probenoberfläche löst. Das angeregte System bestehend aus Blattfeder, Messspitze und Oberfläche wird aufgrund der Reibung gedämpft. Die Dämpfung wiederum wird mit dem erwähnten Kontaktmodell aus der Kontaktresonanzkurve bestimmt.

In der Diskussion werden die Ergebnisse beider Messmethoden schließlich miteinander verglichen. Beide Methoden liefern dabei qualitativ gleichwertige Messergebnisse. Zur Interpretation dieser Ergebnisse werden sowohl die Theorie der Reibung als auch die der Probensysteme herangezogen, um so mögliche Dämpfungsprozesse der Reibung in den jeweiligen Systemen zu diskutieren. Damit wird zum einen gezeigt, dass der Phasenübergang der LSMO-Probe zur Folge hat, dass durch die Änderung der magnetischen Ordnung Dissipationskanäle geschlossen werden, was sich in einer Reibungsabnahme äußert. Zum anderen zeigen die Messdaten weitere Eigenschaften, die mit bestehenden Theorien nicht verstanden werden können. Daher sind in diesen Fällen neue Interpretationsmöglichkeiten entwickelt worden. So zeigen die Messdaten der amorphen Probe zum Beispiel eine Dämpfungserhöhung in Abhängigkeit von der Temperatur, die mit einem $\alpha$-Relaxationsprozess in Verbindung gebracht wird. Die LSMO-Probe zeigt zusätzlich eine normalkraftabhängige Reibung bei hohen Temperaturen. Neben theoretischen Arbeiten deuten die Ergebnisse beider Messmethoden dieser Arbeit darauf hin, dass sich aufgrund von elektronischen Eigenschaften eine Deckschicht an der Oberfläche der LSMOProbe ausbildet, die ähnlich wie die magnetische Ordnung Einfluss auf die Reibung hat und so eine andere Reibung zeigt als das Bulk-Material.

Den Abschluss bilden eine Zusammenfassung dieser Dissertation und ein Ausblick auf mögliche Fortführung der Untersuchung der Reibung auf Basis der Erkenntnisse, die diese Arbeit liefert. 


\section{Tribologie}

Der Begriff Tribologie (dt. Reibungslehre) - von altgriechisch tpí $\omega \omega$ (tribo dt. reiben, abnutzen) und 入óros (logos dt. Lehre) - ist erstmals von Peter Jost im Jahr 1966 verwendet worden [Jost66], beschreibt jedoch eine Wissenschaft, die tausende Jahre alt ist. Die Wandmalerei aus dem Grab des Djehutihotep zum Beispiel ist etwa um 1800 v. Chr. entstanden und zeigt, wie 172 Sklaven eine Steinfigur auf einem Schlitten ziehen (s. Abb. 2.1). Zur Reduzierung der Reibung schüttet eine Person eine Flüssigkeit vor den Schlitten. Diese Funktion ist offensichtlich so wichtig, dass die Person auf dem Schlitten stehen darf, während er gezogen wird. Die Untersuchung der Reibung spielt offenbar seit Jahrtausenden eine wichtige Rolle.

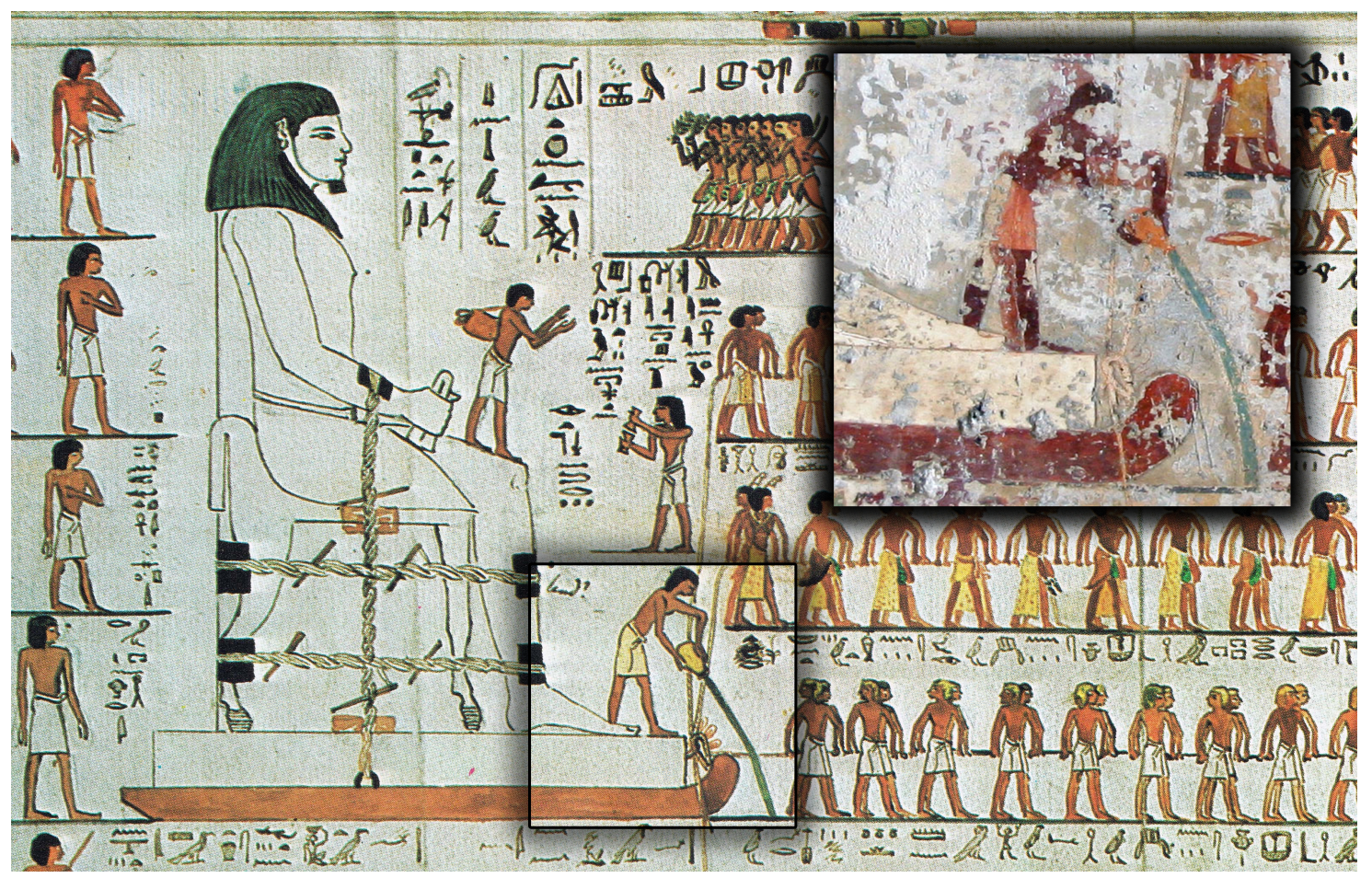

Abbildung 2.1 - Eine Wandmalerei aus dem Grab des Djehutihotep, etwa um $1800 \mathrm{v}$. Chr. entstanden. Sie zeigt 172 Sklaven, die eine Steinfigur ziehen. Zur Reibungsminderung wird eine Flüssigkeit eingesetzt. [Newberry93, Fall14] 
Die Grundlagen der Reibungslehre wurden zu Beginn der Neuzeit von da Vinci, Amontons und Coulomb gelegt. Sie untersuchten Reibung auf makroskopischer Ebene mit eigens dafür entwickelten Messaufbauten. Viele grundlegende Fragen der Reibung aus dieser Zeit sind jedoch bis heute nicht vollständig beantwortet. Um diesen Antworten näher zu kommen, erforscht man Reibung heute mit modernen Geräten auf atomarer Ebene. Im folgenden Kapitel wird ein Überblick über die Entwicklung der Reibungslehre - beginnend in der Neuzeit - gegeben.

\subsection{Tribologie in Der Neuzeit - Historische ENTWICKLUNG}

Leonardo da Vinci $(* 1452, \uparrow 1519)$ war einer der ersten Gelehrten der Neuzeit, der sich ausgiebig mit dem Thema Reibung auseinandergesetzt und sie systematisch studiert hat. Er untersuchte dabei unter anderem die Reibung eines rechteckigen Gegenstands auf einer glatten Oberfläche und leitete den Zusammenhang zur Normalkraft ab, indem er den Reibungskoeffizienten einführte. Da Vinci dokumentierte seine Ideen und Ergebnisse in privaten Notizbüchern. Diese Arbeiten blieben allerdings unveröffentlicht und hatten daher zunächst keinen Einfluss auf die unmittelbar folgenden Untersuchungen zur Reibung. [Bhushan13, Popova15]

Etwa 200 Jahre später - im Jahr 1699 - veröffentlichte der französische Physiker und Statthalter von Lille Guillaume Amontons (*1663, †1705) seine Arbeit zur Reibung (s. auch Abb. 2.2), in der er die folgenden Grundlagen formulierte: [Amontons99, Popova15]

Der Widerstand, der durch die Reibung verursacht wird, ...

1. ... ist proportional zur Normalkraft.

2. ... ist gleich für Eisen, Kupfer, Blei und Holz, wenn es mit Öl eingesalbt wurde.

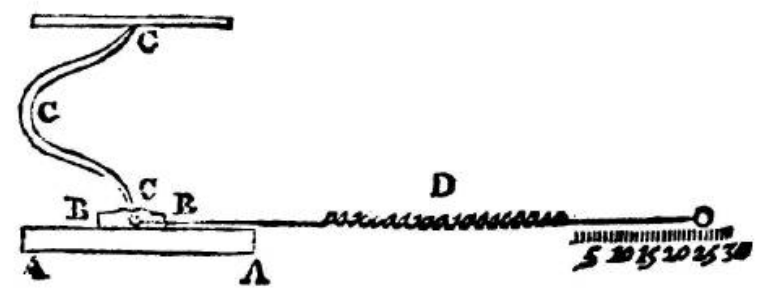

\footnotetext{
Abbildung $2.2-$ Amontons' Messaufbau. Eine Masse wirkt über die Balkenfeder $C$ auf die Fläche $B$, die auf Fläche A liegt. Über die Auslenkung der Feder D kann die Haftreibung bestimmt werden. Nach [Amontons99].
} 
3. ... ist etwa ein Drittel der Normalkraft.

4. ... ist unabhängig von der Geschwindigkeit.

5. ... ist unabhängig von der Kontaktfläche der reibenden Gegenstände.

Auch wenn über den Wert „ein Drittel der Normalkraft“ schon zu Amontons' Zeit gestritten wurde, sind seine Formulierungen zur Reibung allgemein als gültig und wahr angenommen worden, so dass wir die Punkte 1 und 5 auch heute noch als erstes bzw. zweites Amontons'schen Gesetz kennen (s. Seite 9).

1725 veröffentlichte der nach England geflohene französische Naturphilosoph John Theophilus Desaguliers $\left({ }^{*} 1683\right.$, †1744) Arbeiten, die zum ersten Mal Amontons' Ideen angriffen. Desaguliers verpresste händisch zwei Bleikugeln und untersuchte die Kohäsion, indem er die Kraft maß, die er brauchte, um sie wieder zu trennen (s. Abb. 2.3). Vor der Verpressung ist eine glatte Kontaktfläche geschaffen worden, indem er kleine Kugelsegmente entfernte. Desaguliers beobachtete, dass nach jeder Trennung die zur Trennung benötigte Kraft größer wurde, die Kontaktfläche jedoch gleich blieb. Auch das Reinigen der Kontaktfläche führte zur Erhöhung der benötigten Kraft. Desaguliers schlussfolgerte, dass die Kohäsion - und daher auch die Reibung - von der Form des Kontaktes abhängig ist: ein Widerspruch zum zweiten Amontons'schen Gesetz. [Desagulier24]

Der französische Physiker Charles Augustin de Coulomb $(* 1736, \dagger 1806)$ [Gillmor71, Popova15] ist heute vor allem für seine Arbeiten zum Thema der Elektrostatik und wegen der nach ihm benannten Einheit der elektrischen Ladung bekannt. Aber auch er widmete der Untersuchung der Reibung viel Zeit und veröffentlichte viele Artikel zu diesem Thema (s. z. B. [Coulomb73, Coulomb97, Coulomb01, Coulomb21]). Seine umfangreichen Experimente diesbezüglich zeigen, wie komplex Reibung ist. Nach Coulomb hängt die Reibung von deutlich mehr Parametern ab, als Amontons glaubte, nämlich von [Gillmor71]:

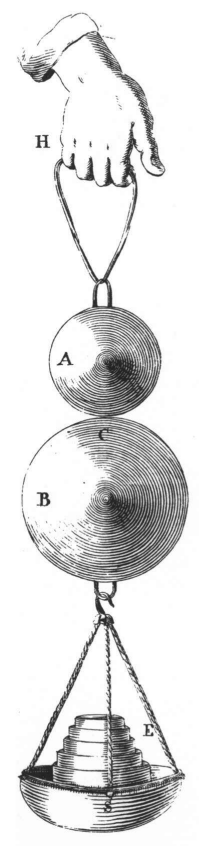

Abbildung 2.3 - Desaguliers verpresste händisch zwei Bleikugeln miteinander und untersuchte, wie viel Masse $E$ es benötigt, um die Kugel zu trennen. Aus seinen Entdeckungen schloss er auf einen Widerspruch zu Amontons' Ergebnissen. Nach [Desagulier24]. 
- Geschwindigkeit

- Kontaktfläche

- Gewicht (Normalkraft)

- Schmiermittel

- Material, das zwischen reagierenden Körpern entsteht

- Oberflächeneigenschaften (glatt, rau)

- Verformungs- oder Kohäsionseffekte durch Ruhezeiten

- Geometrische Orientierung der Oberflächen

- Deformation aufgrund der Geometrie der Oberflächen (Form der interagierenden Oberflächen - planar, spitz, abgerundet)

- Temperatur, Luftfeuchtigkeit und Luftdruck

- Art der Bewegung (gleichförmig oder impulsiv)

Wohl auch aufgrund der Anzahl dieser Parameter formulierte Coulomb jedoch nur sehr allgemeine Zusammenfassungen der Reibung [Coulomb21, S. 99f]:

1. Nach einer gewissen Zeit ist die Reibungskraft zwischen Holz und Holz direkt proportional zur Normalkraft. Die Reibungskraft nimmt nur in den ersten Momenten des Kontakts zu, nach ein paar Minuten erreicht sie ihr Maximum.

2. Die Reibungskraft zwischen Holz und Holz ist auch für unterschiedliche Geschwindigkeiten proportional zur Normalkraft. Die Haftreibung ist jedoch stets größer. Man findet zum Beispiel, dass die Kraft, die benötigt wird, um den Kontakt zu lösen, im Vergleich zur Reibungskraft bei einer bestimmten Geschwindigkeit das Verhältnis 9,5 zu 2,2 hat.

3. Die Reibungskraft zwischen Metall und Metall ist ebenfalls proportional zur Normalkraft, allerdings ist sie genauso groß wie die der Haftreibung.

4. Für verschiedene Oberflächen wie Holz auf Metall unterscheiden sich die Ergebnisse von den vorherigen. Die Intensität der Reibung nimmt dabei langsam zu und erreicht ihr Maximum nach vier bis fünf Tagen - manchmal auch 
erst später. Bei Metall auf Metall hingegen passiert dies instantan, bei Holz auf Holz innerhalb weniger Minuten. Der Reibungskraftanstieg zwischen Holz und Metall ist dabei so langsam, dass Reibungsmessungen bei langsamen Geschwindigkeiten in etwa der Haftreibung entsprechen. Im Gegensatz zu Metall auf Metall oder Holz auf Holz steigt die Reibung mit der Geschwindigkeit an.

Coulomb konnte so anhand umfangreicher experimenteller Ergebnisse Amontons' Gesetze bestätigen, zeigte aber gleichzeitig deren Grenzen auf. Zusammenfassend können so drei Gesetze der Reibung gegeben werden, die auch heute noch in jedem Lehrbuch zur Reibung zu finden sind:

\section{Amontons'sches Gesetz}

Die Reibungskraft $F_{\mathrm{F}}$ ist direkt proportional zur Normalkraft $F_{\mathrm{N}}$.

\section{Amontons'sches Gesetz}

Die Reibungskraft $F_{\mathrm{F}}$ ist unabhängig von der Kontaktfläche.

\section{Coulomb'sches Gesetz}

Die Reibungskraft $F_{\mathrm{F}}$ ist unabhängig von der Geschwindigkeit.

Dabei ist üblicherweise der Reibungskoeffizient definiert als

$$
\mu=\frac{F_{\mathrm{F}}}{F_{\mathrm{N}}} \text {. }
$$

Es fehlte allerdings immer noch eine mikroskopische Erklärung der Reibung. Coulomb glaubte zunächst, Kohäsion wäre der Ursprung der Reibung, trennte sich allerdings wieder von dieser Idee, da die Reibung dann proportional zur Kontaktfläche sein müsste, was seinen eigenen Messungen widersprach. Daher stellte sich Coulomb eine gewisse Verzahnung der Oberflächen vor (s. Abb. 2.4), die sich unter Last vergrößert. Er konnte aber noch keine topographischen Bilder der Oberflächen erstellen, um diese Idee zu beweisen, da die nötige Technik fehlte.

Abbildung 2.4 - Coulomb stellte sich eine Verzahnung der Oberflächen vor, die zur Erhöhung von Reibung führen könnte. Nach [Gillmor71].

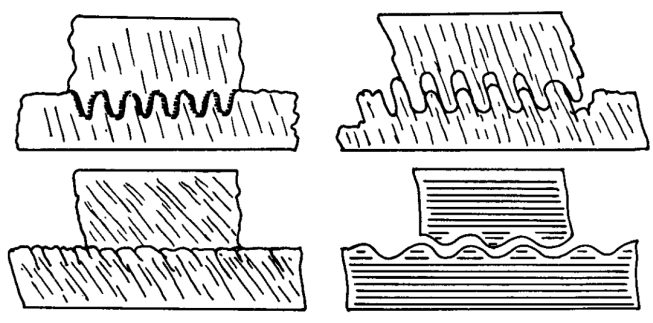




\subsection{Tribologie im 20. Jahrhundert - Der Weg ZUR MikRoskALA}

Dies änderte sich mit der Entwicklung von Taststiftmethoden und des Elektronenmikroskops. In der ersten Hälfte des 20. Jahrhunderts gelang es Bowden und Tabor - auch mit Hilfe solcher Instrumente - die kontraintuitive Vermutung, dass die Reibung unabhängig von der Kontaktfläche sein soll, zu erklären. Sie postulierten, dass die tatsächliche Kontaktfläche nicht der scheinbaren Kontaktfläche entspricht. Gemessene Oberflächenprofile zeigten deutlich, dass glatte Oberflächen nicht existieren. Zwei Körper, die sich berühren, berühren sich nur an wenigen Punkten. Wird der Druck erhöht, entstehen mehr dieser Kontakte und die tatsächliche Kontaktfläche wird größer, ohne dass sich die scheinbare makroskopische Kontaktfläche ändert (vgl. Abb. 2.5).
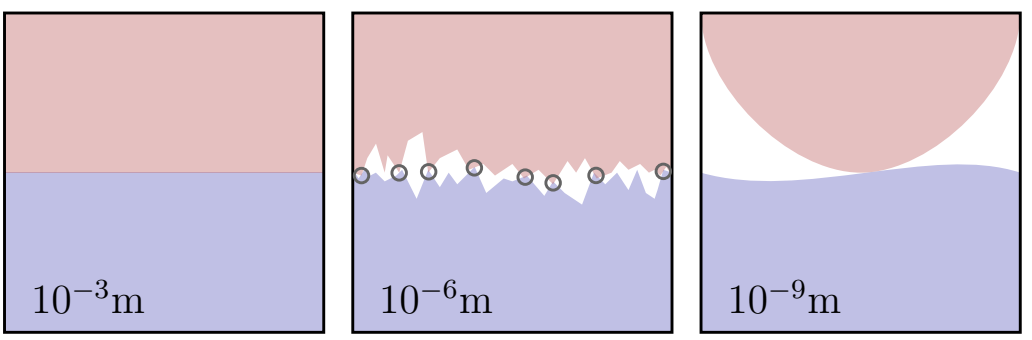

Abbildung 2.5 - Zwei makroskopisch glatte Körper berühren sich nur an wenigen punktförmigen Einzelkontakten (engl. Single-Asperities). Erhöht man den Druck, entstehen mehr dieser Kontakte und die tatsächliche Kontaktfläche wird größer, ohne dass sich die scheinbare, makroskopische Kontaktfläche ändert. Nach [Hölscher08].

Das Reibungsmodell von Bowden und Tabor nimmt an, dass die „wirkliche Berührungsfläche in den meisten praktischen Fällen für alle Arten und Formen von Rauigkeiten sich beinahe proportional zur Belastung ändert" [Bowden59] und gegeben ist über $p_{\mathrm{m}} A=F_{\mathrm{N}}$, mit dem mittleren Fließdruck $p_{\mathrm{m}}$. Außerdem beschreibt es die Reibungskraft als

$$
F_{\mathrm{F}}=S+P
$$

wobei $S=A \tau-$ mit der Scherfestigkeit $\tau$ - die Kraft ist, die zur Abscherung der Kontakte erforderlich ist und $P$ die Kraft, die zur Furchenbildung, also zur Materialverdrängung, nötig ist. Letztere wird üblicherweise vernachlässigt, so dass sich schließlich

$$
F_{\mathrm{F}}=\frac{\tau}{p_{\mathrm{m}}} F_{\mathrm{N}}
$$


ergibt. Die Reibungskraft ist also auch hier eine Funktion der Normalkraft und unabhängig von der scheinbaren Kontaktfläche und somit übereinstimmend mit den Gesetzen von Amontons. [Bowden42, Bowden59]

Die Arbeitsgruppe um Tabor entwickelte 1969 ein Oberflächenkraftmessgerät [Tabor69] (engl. surface force apparatus, SFA) mit dem es möglich ist, Distanzen von $0,1 \mathrm{~nm}$ und Kräfte um $10 \mathrm{nN}$ zu messen. Mit diesem Aufbau gelang es erstmals, Reibung mit sehr kleinen Normalkräften zu messen. Israelachvili und Tabor beobachteten dabei, dass bei ausreichend kleinen Normalkräften keine Abscherung auftritt. [Israelachv72, Israelachv73] Dies wiederum steht im Widerspruch zur Grundidee des Reibungsmodells von Bowden und Tabor. Was also ist die physikalische Grundlage des Energieübertrags im Reibungsprozess, wenn keine Materialverformung vorliegt?

Um der Antwort zu dieser Frage näher zu kommen, muss man noch genauer messen. Bowden und Tabor sprachen von vielen einzelnen halbkugelförmigen Kontaktrauheiten [Bowden42, Tabor57], die die wahre Kontaktfläche ausmachen. Um nun die zu Grunde liegenden physikalischen Eigenschaften der Reibung zu verstehen, muss solch ein einzelner Kontakt experimentell sowie analytisch untersucht werden.

\subsection{Moderne Tribologie - Reibung auf Der NANOSKALA}

Mate et al. legten 1987 mit der Entdeckung des Stick-Slip-Effekts ${ }^{1}$ auf atomarer Ebene den Grundstein zur Erforschung der Reibung auf der Nanoskala [Mate87]. Auch wenn es nicht das Ziel dieser Arbeit war, den physikalischen Ursprung der Reibung zu finden, ist sie gewissermaßen der Ausgangspunkt der Nanotribologie [Krylov14]. Stick-Slip-Bewegungen sind auch auf der Makroskala bekannt. Beispiele aus dem Alltag sind quietschende Türen oder stridulierende Grashüpfer. Da dieses Phänomen offenbar universell über mehrere Größenordnungen auftritt, sollen nun bevor eventuelle physikalische Dissipationsprozesse beschrieben werden - einfache Modelle vorgestellt werden, die die Stick-Slip-Bewegung beschreiben.

\footnotetext{
${ }^{1}$ Eine Stick-Slip-Bewegung zeichnet sich durch abrupte Beschleunigungsänderungen auf. Man „klebt" in einer Position und „springt" plötzlich auf die nächste.
} 


\subsubsection{Prandth-TomLinson-Modell}

In einem Gedankenexperiment beschrieb Prandtl 1928 eine kinetische Theorie der festen Körper, die auch auf atomarer Ebene angewendet werden kann. Auch wenn man dieses Modell teilweise immer noch unter dem historisch inkorrekten Namen „Tomlinson-Modell" findet, verwendet man heute üblicherweise nur noch den Namen Prandtl-Tomlinson-Modell und das, obwohl die im Kontext zitierte Veröffentlichung Tomlinsons das Modell gar nicht enthält. Anhand dieses simpel anmutenden Modells sollen grundlegende atomare Reibungsprozesse verstanden werden.

\section{STICK-SLIP-VERHALTEN}

Abbildung 2.6 zeigt das Grundprinzip des Prandtl-Tomlinson-Modells schematisch [Prandt128, Tomlinson29]. Ein einzelnes Atom (rot) ist über eine Feder mit Federkonstante $k$ an einem Gleiter (grau) befestigt und wird mit konstanter Geschwindigkeit $\vec{v}$ über eine Oberfläche gezogen. Dieses formt aufgrund der Gitteratome (blau) mit dem Abstand $a$ ein periodisches Potential $U_{\mathrm{O}}$ mit der Amplitude $U_{0}$. In der Anwendung ist das Prandtl-Tomlinson-Modell auch für mehratomige Kontakte gültig ${ }^{2}$, da die Periodizität der Oberfläche gegeben bleibt [Krylov14]. Das Potential

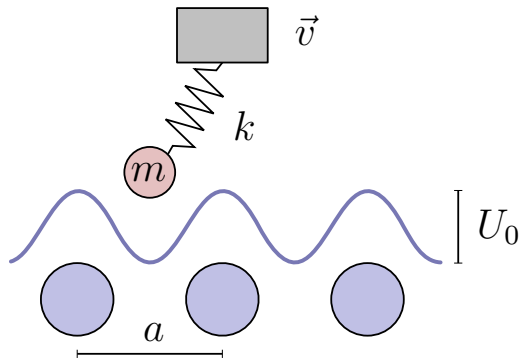
Abbildung 2.6 - Das Prandtl-Tomlinson-Modell beschreibt die Kräfte, die aufgrund eines Ober- flächenpotentials $U_{O}$, resultierend aus periodisch angeordneten Atomen (blau) mit dem Abstand $a$, auf ein einzelnes Teilchen (rot) wirken, das mit konstanter Geschwindigkeit $\vec{v}$ über die Oberfläche gezogen wird. [Prandt128, Tomlinson29].

des gesamten Systems ergibt sich aus der Summe von sinusförmigem Oberflächenpotential $U_{\mathrm{O}}$ und potentieller Energie $U_{\mathrm{F}}$ der Feder:

$$
U_{\mathrm{g}}=U_{\mathrm{O}}+U_{\mathrm{F}}=\frac{U_{0}}{2}\left[1-\cos \left(\frac{2 \pi x}{a}\right)\right]+\frac{k}{2}(x-\vec{v} t)^{2} .
$$

Dabei beschreibt $x$ die Position des einzelnen Atoms und $\vec{v} t$ die Position des Gleiters. In einer Reibungsmessung bestimmt man die Federkraft bzw. Lateralkraft, die

\footnotetext{
${ }^{2}$ Eine Erweiterung des Modells, die solche Kontakte genauer betrachtet, indem sie weitere Atome am Gleiter berücksichtigt, die zusätzlich Kräfte untereinander spüren, ist das FrenkelKontorova-Tomlinson-Modell [Weiss96].
} 
hier durch

$$
F_{\mathrm{F}}=-k(x-\vec{v} t)
$$

gegeben ist.

Während der Gleiter mit konstanter Geschwindigkeit gezogen wird, verweilt das Atom solange in einem lokalen Minimum des Oberflächenpotentials, bis die Kraft der Feder groß genug ist, um das Atom über die Energiebarriere ins nächste Potentialminimum der Oberfläche zu ziehen. Abbildung 2.7 zeigt das Potential aus Gleichung 2.4 für drei verschiedene Zeitpunkte. Bei $\vec{v} t=3,5 \AA$ sitzt das Atom noch bei $x \approx 0 \AA$ (blauer Kreis). Bei $\vec{v} t=5,5 \AA$ ist die Kraft der Feder so groß, dass das Potential an dieser Stelle kein lokales Minimum mehr zeigt und das Atom fällt von $x \approx 0 \AA$ (grauer gestrichelter Kreis) auf $x \approx 2 \AA$ (grauer Kreis). Noch später bei $\vec{v} t=7,5 \AA$ geschieht der gleiche Prozess noch einmal und das Atom fällt auf Position $x \approx 5 \AA$ (roter Kreis). Das Atom verweilt (stick) also immer eine kurze Zeit auf einer Position und springt (slip) dann um etwa die Distanz $a$ in die nächste.

\begin{abstract}
Abbildung 2.7 - Das PrandtlTomlinson-Modell nach Gl. 2.4 mit den Werten $U=0,5 \mathrm{eV}$, $k=2 \mathrm{~N} \mathrm{~m}^{-1}$ und $a=0,25 \mathrm{~nm}$. Das periodische Oberflächenpotential führt zum Stick-SlipVerhalten. Ein Teilchen im Potential verweilt kurz auf einer Position, bis die Federkraft groß genug ist, um es in das nächste Minimum zu befördern.
\end{abstract}

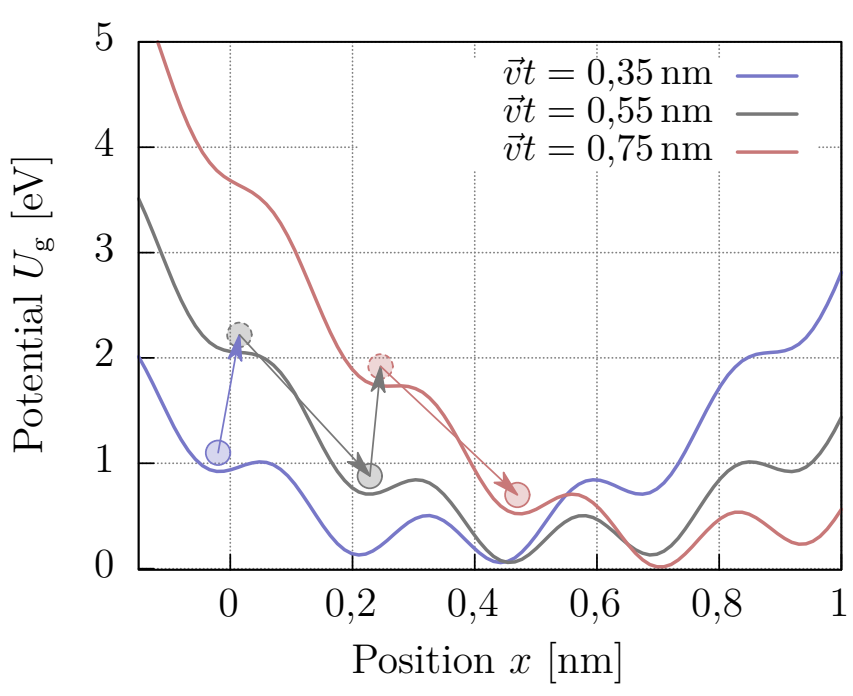

Mit diesem simplen Modell ist es möglich, einfache Reibungsmessungen zu simulieren. Dabei wird allerdings klar, dass das Messsystem großen Einfluss auf die Messung hat [Tománek91]. Abbildung 2.8 a) zeigt drei Simulationen der Lateralkraft $F_{\mathrm{F}}$ in Abhängigkeit von der Gleiterposition für unterschiedlichen Federkonstanten $k$. Da ein Hüpfen ins nächste Minimum geschieht, wenn die Federkraft größer wird als die Kraft vom Oberflächenpotential, entspricht die Steigung von $F_{\mathrm{F}}$ der Federkonstanten $k$. Es ist zu sehen, dass die Maximalwerte der Lateralkraft 
für alle Federkonstanten bei $1 \mathrm{nN}$ liegen. Der Durchschnittswert $\bar{F}_{\mathrm{F}}$ - dargestellt durch die horizontalen gestrichelten Linien - ist allerdings abhängig von der Federkonstanten. Je größer die Federkonstante ist, desto kleiner wird die gemessene Kraft. Das kann so weit gehen, dass die Spitze dem Gleiter vorauseilt, so dass auch negative Lateralkräfte beobachtet werden können. In diesen Fällen geht der Mittelwert der Lateralkräfte gegen 0 und man spricht von Superlubrizität (vgl. Abb. 2.8 b)). Der Übergang von einer Stick-Slip-Bewegung zum Gleiten geschieht, wenn das Potential $U_{\mathrm{g}}$ nur noch ein Minimum aufweist - und so ein Hüpfen nicht mehr möglich ist. Das geschieht, wenn die zweite Ableitung $\mathrm{d}^{2} U_{\mathrm{g}} / \mathrm{d} x^{2}>0$ ist. Für einen dimensionslosen Parameter $\alpha$ gilt daher im Fall der Superlubrizität

$$
\alpha:=\frac{2 \pi^{2} U_{0}}{k a^{2}} \leq 1
$$

In Abbildung 2.8 b) ist der Bereich eingezeichnet, für den diese Bedingung gültig ist. Wie man sieht, stimmt er mit den simulierten Daten überein. Experimentell ist der Übergang von Stick-Slip zur Superlubrizität ebenfalls nachgewiesen worden [Gnecco00, Dienwiebel04, Socoliuc04].

a) Slip-Stick-Verhalten

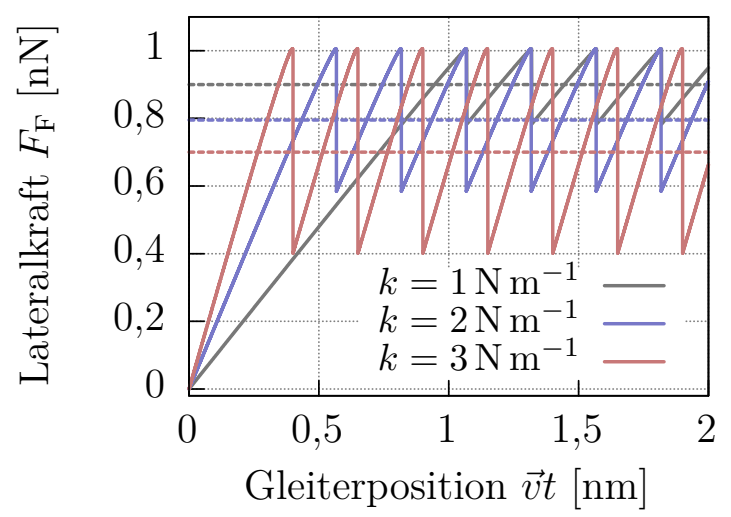

b) Einfluss von $k$

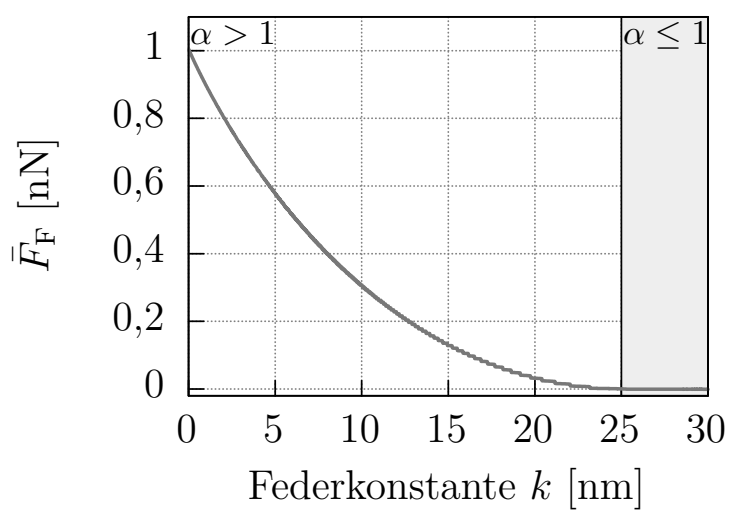

Abbildung 2.8 - Simulation einer Bewegung im Prandtl-Tomlinson-Modell mit den Werten $U=0,5 \mathrm{eV}, k=2 \mathrm{~N} \mathrm{~m}^{-1}$ und $a=0,25 \mathrm{~nm}$. a) Bewegt man den Gleiter des Prandtl-Tomlinson-Modells mit konstanter Geschwindigkeit, beobachtet man ein Stick-Slip-Verhalten, das auch von der Federkonstanten $k$ abhängt. b) Der maximale Wert der Lateralkraft ist für alle $k$ gleich, der Mittelwert $\bar{F}_{F}$ nimmt jedoch mit zunehmendem $k$ ab. Ist $\alpha \leq 1$ spricht man auch von Superlubrizität. 


\section{THERMische EFFEKTE}

Im Prandtl-Tomlinson-Modell wird die aufgebrachte Energie des Gleiters nach jedem Sprung abrupt und komplett dissipiert, so dass man eine Reibungshysterese zwischen Hin- und Rückrichtung (s. Kap. 3.2.2) verstehen kann. Allerdings ist diese Form der Energiedissipation unabhängig von Temperatur, Geschwindigkeit oder anderen physikalischen Dissipationsprozessen. Es liegt also nahe, einen Dämpfungsterm zu integrieren, ohne zunächst auf dessen tatsächlichen physikalischen Ursprung einzugehen und außerdem thermische Effekte zuzulassen. Thermische Effekte können als zufällig auftretende Kraft $L(t)$ eingeführt werden, so dass sich für das System die Bewegungsgleichung

$$
m \ddot{x}+\eta m \dot{x}=\frac{\mathrm{d} U_{\mathrm{g}}}{\mathrm{d} x}+L
$$

ergibt, wobei $L$ stochastisches Verhalten ohne Korrelation über die Zeit zeigt, so dass $\langle L(T)\rangle=0$ und über das Fluktuations-Dissipations-Theorem einem weißen Rauschen $\left\langle L(t) L\left(t^{\prime}\right)\right\rangle=2 m \eta k_{\mathrm{B}} T \delta\left(t-t^{\prime}\right)$ entspricht [Vollmer79, Risken84, Müser03]. Dabei bezeichnet $T$ die Temperatur, $k_{\mathrm{B}}$ die Boltzmann-Konstante, $m$ die Masse und $\eta$ einen Dämpfungsterm.

Aufgrund der thermischen Energie können die Energiebarrieren des Potentials $\Delta E_{i}=U_{\mathrm{g}, i}\left(x_{\max , i}\right)-U_{\mathrm{g}, i}\left(x_{\min , i}\right)-$ also die Energiedifferenz zwischen lokalem Minimum $U_{\mathrm{g}, i}\left(x_{\min , i}\right)$ und anliegendem Maximum $U_{\mathrm{g}, i}\left(x_{\max , i}\right)$ - überwunden werden, bevor $\Delta E_{i}=0$ ist. Dies kann auch als zusätzliche instantane laterale Kraft $F_{\mathrm{F}}$ angesehen werden [Riedo03]. Die Bewegungsgleichung 2.7 kann deutlich vereinfacht werden, wenn angenommen wird, dass der Abstand der instantanen thermischen Energiesprünge im Vergleich zur Scangeschwindigkeiten klein ist [Krylov05]. Dann fluktuiert die thermische Energie nach der Boltzmann-Statistik. Dabei muss bedacht werden, dass auch Sprünge in höhere Energien möglich sind. Betrachtet man zunächst den einfacheren Fall, bei dem nur Sprünge in tiefere Niveaus zugelassen werden, ergibt sich für die Wahrscheinlichkeit $p(t)$ bzw. $p\left(F_{\mathrm{F}}\right)$, das Teilchen im niedrigeren Niveau zu finden [Gnecco00],

$$
\begin{gathered}
\frac{\mathrm{d} p(t)}{\mathrm{d} t}=-f_{0} \exp \left[-\frac{\Delta E_{i}(t)}{k_{\mathrm{B}} T}\right] p(t) \\
\frac{\mathrm{d} p\left(F_{\mathrm{F}}\right)}{\mathrm{d} F_{\mathrm{F}}} \frac{\mathrm{d} F_{\mathrm{F}}}{\mathrm{d} t}=-f_{0} \exp \left[-\frac{\Delta E_{i}\left(F_{\mathrm{F}}\right)}{k_{\mathrm{B}} T}\right] p\left(F_{\mathrm{F}}\right) .
\end{gathered}
$$

Dabei ist die charakteristische Frequenz des Systems in der Größenordnung von 
$f_{0} \approx \sqrt{U_{0} / m a^{2}}$ [Müser03]. Die Energiedifferenz $\Delta E$ kann abgeschätzt werden, indem man von $\partial U_{\mathrm{g}} / \partial x$ eine $\partial U_{\mathrm{g}} / \partial x$ Taylor-Reihe zweiter Ordnung entwickelt, für die sich [Riedo03, Müser03]

$$
\Delta E(t)=\frac{\sqrt{8}}{3}\left(E_{0}-\frac{k v t a}{\pi}\right)^{3 / 2}
$$

ergibt. Löst man nun Gleichung 2.8, ergibt sich die Beziehung [Sang01, Persson03, Riedo03, Hölscher08, Jansen10]

$$
\bar{F}_{\max }=F_{0 \mathrm{~K}, \max }-\left[\beta k_{\mathrm{B}} T \ln \left(\frac{v_{\mathrm{c}}}{v}\right)\right]^{2 / 3} \text {. }
$$

Dabei wird der Parameter $\beta$ durch die Form des Potentials bestimmt. Für ein sinusförmiges Potential $U_{\mathrm{g}}$ ergibt sich

$$
\beta=\frac{3 \pi}{2} \sqrt{\frac{F_{0 \mathrm{~K}, \max }}{2} .}
$$

Deutlich unterhalb der kritischen Geschwindigkeit $v_{\mathrm{c}}=f_{0} k_{\mathrm{B}} T / \sqrt{2} k a$ nimmt die Reibung nahezu logarithmisch zu. Nähert man sich jedoch der Geschwindigkeit $v_{\mathrm{c}}$, geht $\left(\ln v_{\mathrm{c}} v^{-1}\right)^{2 / 3}$ schneller gegen 0, so dass der mittlere Wert der Reibungspeaks $\bar{F}_{\text {max }}$ nahe der kritischen Temperatur immer schneller gegen den Wert $F_{0 \text { K,max }}$ ohne thermische Einflüsse strebt. Erinnert man sich daran, dass die thermischen Effekte in Gleichung 2.7 eigentlich instantane $\delta$-Impulse sind, kann der Effekt der Scangeschwindigkeit anschaulich verstanden werden. Je schneller gescannt wird, desto weniger dieser Impulse treten auf und desto weniger Einfluss nehmen thermische Effekte. Die Temperatur $T$ fließt hier zum einen direkt in Gleichung 2.10 ein, wo sie proportional zur Reibungsabnahme ist. Zum anderen ist sie proportional zur kritischen Temperatur. Die $\left(\ln v_{\mathrm{c}} v^{-1}\right)^{2 / 3}$-Abhängigkeit ist von Sang et al. [Sang01] über einen großen Geschwindigkeitsbereich gezeigt worden, was gleichzeitig bedeutet, dass eine häufig - auch phänomenologisch - angenommene logarithmische Abhängigkeit wie in [Prandt128, Heslot94, Gnecco00, Evans01] nur in bestimmten Geschwindigkeitsbereichen genau genug ist.

Sehr langsame Scangeschwindigkeiten führen zu vielen thermisch aktivierten Barriereüberwindungen. Daher kann man in diesem Fall beobachten, dass die Reibung abnimmt. Es wird dabei von Thermolubrizität gesprochen, da die Temperatur hier wie ein Schmiermittel fungiert [Krylov05, Jinesh08]. Die Reibung ist in diesem 
Fall

$$
\bar{F} \sim m \eta v \frac{U_{0}}{k_{\mathrm{B}} T} \exp \left(\frac{U_{0}}{k_{\mathrm{B}} T}\right) .
$$

Abbildung 2.9 zeigt eine einfache Simulation der thermischen Effekte, bei der das Oberflächenpotential $U_{0}$ vor jedem Simulationsschritt zufällig nach $U_{0}=U_{0}(1-\xi \eta)$ geändert wird. Dabei ist $\eta$ ein fester Prozentwert - in Abb. $2.9 \eta=0 \%, 5 \%$ und $10 \%$ - und $\xi$ eine zufällige Zahl zwischen 0 und 1. Es fällt auf, dass die Lateralkraft ein Rauschen zeigt, das man auch in realen Messungen beobachtet, aber auch, dass die mittlere Lateralkraft kleiner wird. Durch thermisches Hüpfen wird die Auslenkung der Feder früher reduziert.

Abbildung 2.9 - Das Prandtl-

Tomlinson-Modell nach Gl. 2.4 mit $U=0,5 \mathrm{eV}(1-\xi \eta), k=$ $2 \mathrm{~N} \mathrm{~m}^{-1}$ und $a=0,25 \mathrm{~nm}$. Thermische Effekte werden als zufällig Variation mit einer zufälligen Zahl $0 \leq \xi \leq 1$ und der Stärke $\eta$ berücksichtigt.

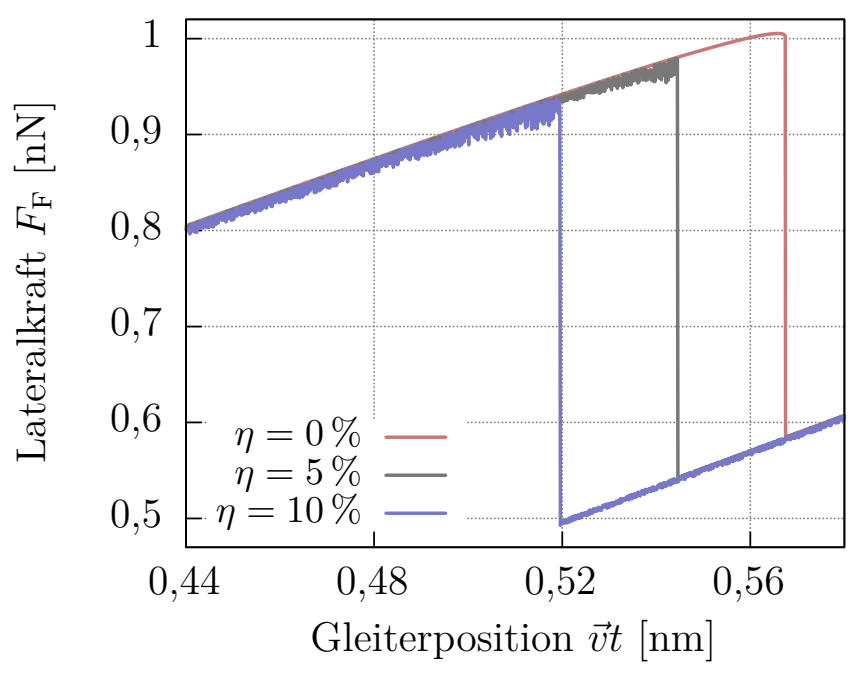

\section{Mechanische Anregung}

Neben thermischem Überwinden der Energiebarrieren gibt es auch die Möglichkeit, mechanische Anregungen der Spitze dazu zu nutzen. Kerssemakers et al. regten laterale Kontaktresonanzen während der Reibungsmessung an und konnten so Reibung reduzieren [Kerssemake98]. Socoliuc et al. nutzten eine ähnliche Methode, bei der die Spitze rechtwinklig zur Probenoberfläche (out-of-plane) angeregt wird. Sie konnten so die Reibung im Rahmen der Messgenauigkeit komplett unterdrücken [Socoliuc06]. Um die mechanische Anregung auch im Prandtl-Tomlinson-Modell zu untersuchen, wird es angepasst, indem die periodische out-of-plane-Bewegung des Gleiters als periodische Änderung des Oberflächenpotentials

$$
U_{\mathrm{O}}(t)=U_{0}(1+A \cos (2 \pi f t))
$$


a) Mechanischer Effekt

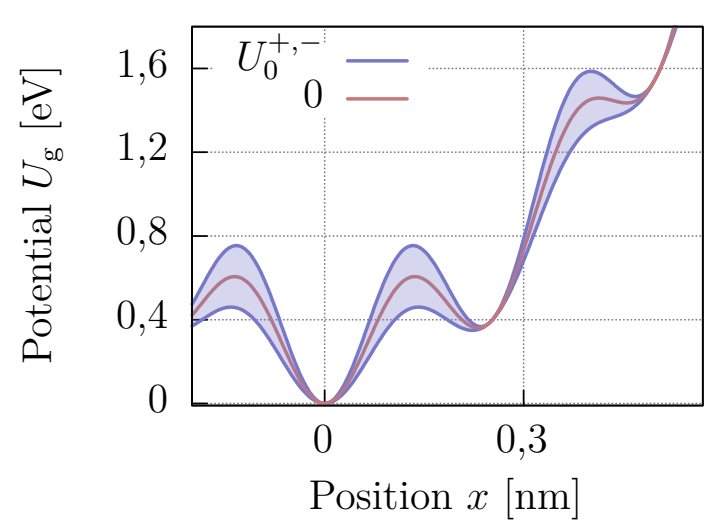

b) Simulation mit Anregung

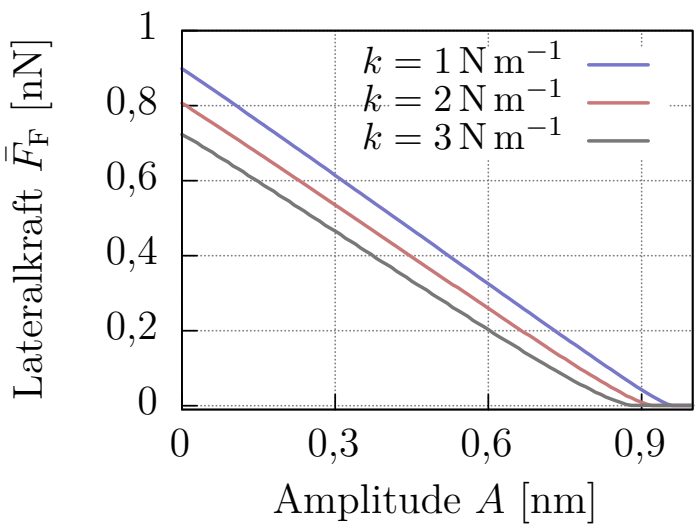

Abbildung 2.10 - Externe Anregungen werden im Prandtl-Tomlinson-Modell durch zeitliche Variation des Potential simuliert. a) Wird das Potential wie durch die blaue Fläche dargestellt variiert, können Energiebarrieren einfacher überwunden werden. b) Je größer die Amplitude A der Anregung ist, desto weniger Energie ist notwendig, um die Potentialbarriere zu überwinden. Bei sehr hohen Amplituden wird die Lateralkraft $\bar{F}_{F}=0$.

dargestellt wird [Socoliuc06, Tizuka09, Fajardo14]. Dadurch ändert sich das periodische Potential wie in Abbildung 2.10 a) dargestellt. Ohne externe Anregung beschreibt die rote Kurve das Potential. Mit Anregung variiert das Potential wie durch die blaue Fläche gekennzeichnet. Die Amplitude $A$ bestimmt dabei die Werte $U_{0}^{+}=U_{0}(1+A)$ und $U_{0}^{-}=U_{0}(1-A)$ und damit Variation der Energiebarriere. Befindet man sich hier zum Beispiel im Minimum bei $x \approx 4,7 \AA$, kann die Energiebarriere aufgrund der Anregung überwunden werden und man fällt auf $x \approx 2,3 \AA$.

Socoliuc et al. regten ihr System mit hohen Frequenzen bei langsamer Scangeschwindigkeit an. Sie nehmen daher an, dass das Potential $U_{\mathrm{O}}$ an jeder Position $x$ mindestens einmal minimiert wird - also mindestens einmal den Wert $U_{0}^{-}$erreicht. In Ungleichung 2.6 kann $U_{0}$ daher ersetzt werden und es ergibt sich die Beziehung

$$
\begin{aligned}
1 & \geq \frac{2 \pi^{2} U_{0}}{k a^{2}}(1-A) \\
\Rightarrow \quad A & \geq 1-\frac{k a^{2}}{2 \pi^{2} U_{0}}=1-\alpha^{-1} .
\end{aligned}
$$

Um die Reibung im Prandtl-Tomlinson-Modell mit mechanischer Anregung zu verringern, helfen große Amplituden, große Federkonstanten, große Atomabstände oder 
Abbildung 2.11 - Die Zwei-Massen-Erweiterung nimmt Rücksicht auf schnelle Bewegungen der Spitze. Zusätzlich zum Prandtl-TomlinsonModell wird hier die Masse $M$ der Blattfeder mit der Federkonstanten $K$ berücksichtigt. Nach [Krylov07].

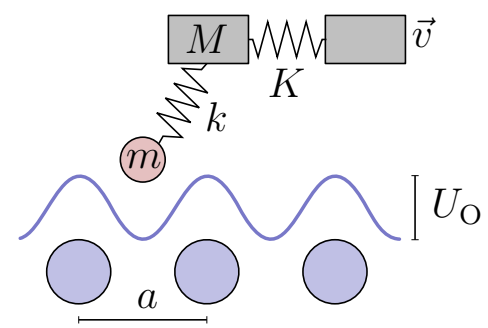

kleine Oberflächenpotentiale. Ist $\alpha^{-1}>1$, befindet man sich wieder im Bereich der Superlubrizität, so dass keine Anregung benötigt wird, um die Reibungskraft zu unterdrücken.

Abbildung 2.10 b) zeigt eine Simulation mit mechanischer Anregung. Die mittlere Lateralkraft $\bar{F}_{\mathrm{F}}$ ist auch ohne Anregung geringer, je größer die Federkonstante $k$ ist, wie es schon in Abbildung 2.8 gezeigt worden ist. Gleichung 2.12 zeigt, dass je größer die Federkonstante ist, desto kleinere Amplituden führen zur Superlubriztät. Auch das zeigt die Simulation, denn die mittlere Lateralkraft geht früher gegen 0 .

\section{Versagen Des PRANdTl-Tomlinson-Modells}

Wie erwähnt steigt im Prandtl-Tomlinson-Modell die Lateralkraft $F_{\mathrm{F}}$ bis zu einem Hüpfen an und die Steigung entspricht der Federkonstanten $k$ (vgl. Abb. 2.8 a). In Messungen des Stick-Slip-Verhaltens auf atomarer Ebene ist die beobachtete Steigung - also die Federkonstante - allerdings mindestens eine Größenordnung kleiner als die der verwendeten Blattfeder [Mate87, Bennewitz99, Gnecco00, Socoliuc04, Dienwiebel04]. Üblicherweise wird angenommen, dass die Messung durch eine effektive Federkonstante $k_{\text {eff }}$ bestimmt ist, die sich aus mindestens zwei Steifigkeiten nach $k_{\text {eff }}=\left(k^{-1}+K^{-1}\right)^{-1}$ zusammensetzt, wobei $K$ die Federkonstante der Blattfeder ist. Dadurch, dass die beobachtete Steifigkeit deutlich kleiner ist als die der Blattfeder, muss die Federkonstante $k$ sehr klein sein, so dass man sie der Spitze zuschreibt [Krylov14]. Das bedeutet, dass das System nicht mehr als zweikomponentig angesehen werden kann, sondern dass Spitze und Blattfeder einzeln betrachtet werden müssen.

Am einfachsten wird dies durch ein Zwei-Massen-Modell beschrieben, dass den Gleiter aus dem Prandtl-Tomlinson-Modell in Spitze und Blattfeder aufteilt (s. Abb. 2.11). Wie auch im Prandtl-Tomlinson-Modell wird ein Atom mit Masse $m$ über ein Oberflächenpotential $U_{\mathrm{O}}$ gezogen, hier allerdings von einer Blattfeder mit der Masse $M$ und der Federkonstanten $K$, die dann erst vom einem Gleiter mit der konstanten Geschwindigkeit $\vec{v}$ gezogen wird. Es gilt $m \ll M$ und $k \ll K$. Die zwei 
Massen in diesem Modell unterscheiden sich um 9-12 Größenordnungen [Krylov14]. Experimentelle und analytische Daten lassen darauf schließen, dass die Masse der Spitze in der Größenordnung von $10^{-18} \mathrm{~kg}$ liegt [Maier05, Abe107, Krylov14]. Die Seitenlänge eines Würfels aus Silizium - das häufig als Spitzenmaterial verwendet wird - wäre bei dieser Masse etwa $100 \mathrm{~nm}$. Typische Spitzenradien liegen im Bereich von einigen zehn Nanometern, so dass diese Masse sinnvoll erscheint.

Die zwei Massen haben auch zur Folge, dass zwei charakteristische Frequenzen beteiligt sind, die sich deutlich unterscheiden. Die Frequenz $f_{\mathrm{F}}$ der Blattfeder liegt im kHz-Bereich, die der Spitze $f_{\mathrm{S}}$ allerdings im hohen GHz-Bereich [Krylov06]. Da die Wahrscheinlichkeit für thermisches Hüpfen proportional zu dieser Frequenz ist (vgl. Gl. 2.8), kann man nun auch verstehen, warum thermische Effekte einen großen Einfluss auf die Reibung haben. Denn im einfachen Prandtl-Tomlinson-Modell wäre die Masse aus Blattfeder und Spitze eigentlich zu groß im Vergleich zu typischen thermisch getriebenen Effekten.

Krylov et al. beschreiben umfangreich, dass das Zwei-Massen-Modell weitere Szenarien einer Reibungsmessung möglich macht [Krylov07]. Je nachdem, welche Werte die beschriebenen Parameter haben, kann es vorkommen, dass die Spitze mit hohen Frequenzen zwischen Energieminima hin und her springt. Die Blattfeder kann dieser Bewegung aber nicht folgen, so dass sie in der Messung üblicherweise verborgen bleibt. Die Messungen von Maier [Maier05] zeigen Energiesprünge unterschiedlicher Zeitskalen. Zum einen gibt es Sprünge, die schneller sind als die Auflösung des Messaufbaus von $15 \mu \mathrm{s}$, zum anderen langsamere von mehreren ms. Krylov et al. interpretieren diese Daten mit schnellen Spitzenbewegungen und langsamen Blattfederreaktionen.

Im Prandtl-Tomlinson-Modell wird nur ein einzelner Kontakt untersucht. Es ist allerdings gezeigt worden, dass reale Kontakte aus mindestens 20 einzelnen Bindungen bestehen. Dabei ist das Formen und das Lösen dieser einzelnen Kontakte jeweils temperatur- und geschwindigkeitsabhängig. In manchen Temperatur- und Geschwindigkeitsbereichen formen sich mehr Kontakte, als sich lösen - oder andersherum. Daher kann es - entgegen dem Prandtl-Tomlinson-Modell - zum Anstieg der Reibung bei tiefen Temperaturen oder zu einer Abweichung vom logarithmischen Verhalten kommen. [Filippov04, Schirmeise06, Barel10a, Barel10b]

\subsubsection{ENERGIEDISSIPATIONSPROZESSE}

Tritt Reibung auf, ist eine Kraft notwendig, um zwei Objekte gegeneinander zu bewegen. Dabei kommt es zu einer Umwandlung von kinetischer und mechanischer 
Energie in Wärme. Das erwärmte System bildet dabei den Endzustand. Die grundlegende Frage ist daher, welche Freiheitsgrade beim Energieübertrag involviert sind und wie die Energie übertragen wird. Diese Frage ist so grundlegend und weitreichend, dass sie bisher nur teilweise beantwortet ist.

Der dominante Prozess der Reibung ist - so wird allgemein angenommen - die Anregung von Gitterschwingungen, die dann die Energie ins System dissipieren lassen. Dies kann je nach System über phononische Prozesse oder im Fall von starker Elektron-Phonon-Kopplung auch über das Elektronensystem geschehen.

\section{Phononische Prozesse}

Persson [Persson00] gibt in einem vereinfachten Bild eine Abschätzung der Energiedissipation über Phononen an: Er nimmt an, dass ein in Eigenfrequenz $f_{0}$ schwingendes Adsorbat bei jeder Oszillation Energie in Form einer Kollision an das Substrat abgibt. Die übertragene Energie $\Delta E=E \cdot\left(\mathrm{m} / \mathrm{m}_{\mathrm{S}}\right)$ hängt dabei von der Energie $E$ der Vibration und der Masse $m$ des Adsorbats sowie der effektiven Masse $m_{\mathrm{S}}$ des Substrats ab. Die effektive Masse ist dabei über den Volumenanteil $\Delta V=m_{\mathrm{S}} / \rho_{S} \propto 8 \pi^{3} c_{\mathrm{S}}^{3} f_{0}^{-3}$ des Substrats bestimmt, der während der Kollisionszeit $\tau \approx 2 \pi f_{0}^{-1}$ beteiligt ist. Mit der transversalen Schallgeschwindigkeit $c_{\mathrm{S}}$ des Substrat ergibt sich dadurch $m_{\mathrm{S}} \propto 8 \pi^{3} \rho_{\mathrm{S}} c_{\mathrm{S}}^{3} f_{0}^{-3}$. Damit ergibt sich für die Dämpfungskonstante $\eta$ aus Gleichung 2.7

$$
\eta \propto f_{0}^{4} \frac{m}{\rho_{\mathrm{S}}}\left(\frac{2 \pi}{c_{\mathrm{S}}}\right)^{3}
$$

Cieplak et al. [Cieplak94, Smith96] interpretierten Messungen von Krim et al., die in mehreren Arbeiten [Krim88, Watts90, Krim90, Krim91a, Krim91b, Tomassone97, Daly96] festgestellt haben, dass einzelne Atomlagen von Krypton oder Xenon auf Silber oder Gold weniger Reibungskraft zeigen als ihre flüssige Phase. Dabei setzen sie die makroskopisch dissipierte Leistung

$$
P=F v=\frac{m \dot{x}}{\tau}
$$

der - über die Zeit integrierte - mikroskopisch dissipierten Leistung

$$
P=\sum_{\mathbf{q}}-i \omega \chi|U(\mathbf{q}, \omega)|^{2}
$$

gleich. Bewegt sich das Adsorbat mit der Geschwindigkeit $v$ über das Oberflächenpotential $U$, dessen Symmetrie durch den reziproken Gittervektor $q$ beschrieben 
wird, oszilliert dies mit der Frequenz $\omega=v q$. Cieplak et al. nehmen nun an, dass die Dämpfung $\chi$ die typische Form eines gedämpften elastischen Mediums mit

$$
\chi^{-1}(\mathbf{q}, \omega)=m\left(\omega_{\mathbf{q}}^{2}-\omega^{2}\right)-i m \omega t_{\mathrm{Ph}}^{-1}
$$

besitzt, wobei $\omega$ die Frequenz und $t_{\mathrm{Ph}}$ die Lebensdauer der Phononen mit Wellenvektor $\mathbf{q}$ ist. Sofern die Geschwindigkeit $v$ deutlich kleiner ist als die Schallgeschwindigkeit $c_{\mathrm{S}}$ und die reziproken Gittervektoren von Substrat $(\mathbf{G})$ und Adsorbat (q) inkommensurabel zueinander sind, gilt für den statischen Strukturfaktor

$$
S(\mathbf{G})=N|\chi(\mathbf{G}, 0) U(\mathbf{G}, 0)|^{2},
$$

mit der Anzahl $N$ der adsobierten Atome.

Dadurch ergibt sich für ein kristallines Substrat schließlich die Beziehung

$$
\tau=t_{\mathrm{Ph}} \frac{N}{2 S(\mathbf{G})} \propto t_{\mathrm{Ph}}
$$

wobei $\tau$ als eine Zeit verstanden werden kann, in der das Adsorbat zum Stoppen kommt und dessen Energie ins Substrat dissipiert. Das führt in diesem Modell zu einer Dämpfung von

$$
\eta=\tau^{-1}=t_{\mathrm{Ph}}^{-1} \frac{2 S(\mathbf{G})}{N} \propto t_{\mathrm{Ph}}^{-1}
$$

\section{Elektronische Reibung}

Auch die erste Abschätzung des elektronischen Anteils an der Reibung kommt von Persson [Persson91, Persson00, Persson95]. Er betrachtet auch hier Adsorbate der Masse $m$, die sich mit einer Geschwindigkeit $v$ über eine Oberfläche bewegen. Dadurch werden Elektronen im Substrat bewegt, die dann über den Widerstand im Substrat Energie dissipieren. Die Dämpfung ist in diesem Fall

$$
\eta_{\mathrm{el}}=\frac{n^{2} e^{2} d}{m n_{\mathrm{A}}} \Delta \rho
$$

Hier ist $n$ die Zahl der Leitungselektronen, $n_{\mathrm{A}}$ die Anzahl der adsorbierten Atome pro Flächeneinheit, $d$ die Filmdicke und $\Delta \rho$ die durch die Adsorbate induzierte Widerstandserhöhung des Films.

Qi zusammen mit Park et al. [Park06, Qi08] haben in verschiedenen Arbeiten Reibung an n-dotiertem Gallium-Arsenit und an p- und n-dotiertem Silizium un- 
Abbildung 2.12 - Reibungsmessung mit Pt-

(a) dotierter Spitze auf n-dotiertem GaAs(100), mit $1 \mathrm{~nm}$ Oxid-Deckschicht, dabei ist eine Fläche von $75 \times 75 \mathrm{~nm}^{2}$ vermessen worden. Zwischen Spitze und Probe wird eine Spannung angelegt. Neben a) der Stromkarte zeigt auch b) die Reibungskarte den Einfluss der Spannung. c) Die gemittelten Werte der Reibung sind höher, sofern ein Strom fließt. Mit freundlicher Genehmigung aus [Qi08].
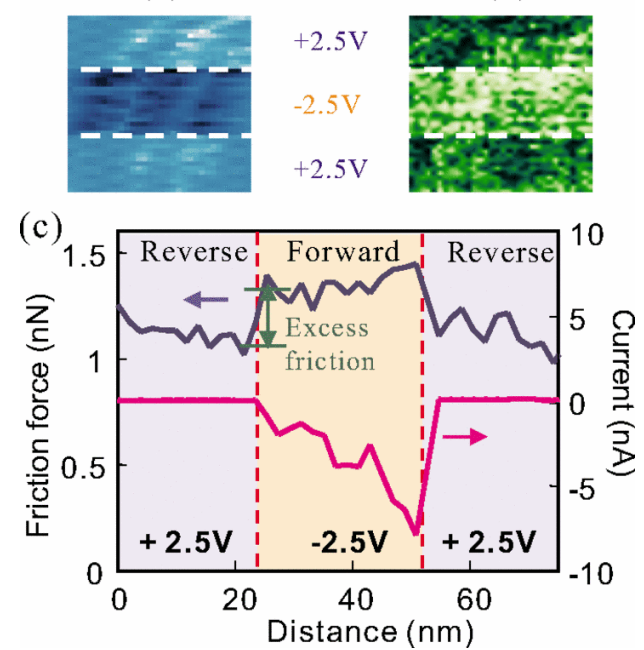

tersucht. Abbildung 2.12 zeigt Reibungsmessungen an n-dotiertem GaAs, das von einer $1 \mathrm{~nm}$ dicken Oxidschicht bedeckt ist. Zwischen Spitze und Probe kann eine Spannung angelegt werden, Abbildung 2.12 a) zeigt eine Stromkarte bei zwei verschiedenen Spannungen. Abbildung 2.12 b) zeigt das zugehörige Reibungssignal. Es ist zu erkennen, dass bei negativer Spannung höhere Reibung gemessen wird. Abbildung 2.12 c) zeigt die gemittelten Messwerte in Abhängigkeit von der Distanz - fließt ein (negativer) Strom, ist die Reibung erhört. Auch bei p- und n-dotiertem Silizium finden sie eine Erhöhung der Reibung, sofern ein elektrischer Strom fließt, also eine negative Spannung am n-dotierten Bereich oder eine positive Spannung am p-dotierten Bereich anliegt. Qi et al. schätzen die Dämpfung mit Hilfe des Drude-Modells über

$$
\eta_{\mathrm{el}}=\frac{2 n e^{2} \rho}{m}
$$

ab und berechnen so einen Wert, der um zwölf Größenordnungen unter dem gemessenen Wert liegt. Um die Ergebnisse zu erklären, postulieren sie, dass die Spitze eine Spur von gefangenen Elektronen in einer Oxidschicht auf der Probe hinterlässt, die wiederum zu Bildladungen in der Spitze und damit zu einer elektrostatischen Anziehung führen. Die von Qi et al. berechnete anziehende Kraft liegt im Bereich der gemessenen.

Üblicherweise wird angenommen, dass die elektronischen Beiträge der Reibung im Vergleich zu phononischen klein sind. Um sie trotzdem möglichst präzise zu messen, haben Stipe et al. [Stipe01] die Dämpfung einer Blattfeder als Funktion des Abstands - also ohne direkten Kontakt zur Probe - gemessen. Sie interpretieren ihre Daten mit fluktuierenden elektrischen Feldern, die wiederum zur Energiedissipation 
über Ohm'sche Verluste durch Bildladungen führen.

Mit gleichem Aufbau konnten Kisiel et al. [Kisiel11] elektronische Reibung von Niob unterdrücken, indem sie die Temperatur soweit reduzierten, dass die Probe in den supraleitenden Zustand überging. Abbildung 2.13 zeigt den gemessenen Reibungskoeffizienten in Abhängigkeit von der - auf die Übergangstemperatur $T_{\mathrm{c}}$ normierte - Temperatur. Ein deutlicher Abfall der Reibung unterhalb $T_{\mathrm{c}}$ ist zu erkennen. Über die angelegte Bias-Spannung zwischen Spitze und Probe konnten sie die gemessene Reibung unterhalb der Sprungtemperatur Phononen zuweisen, die elektronische Reibung ist dort unterdrückt [Volokitin07].

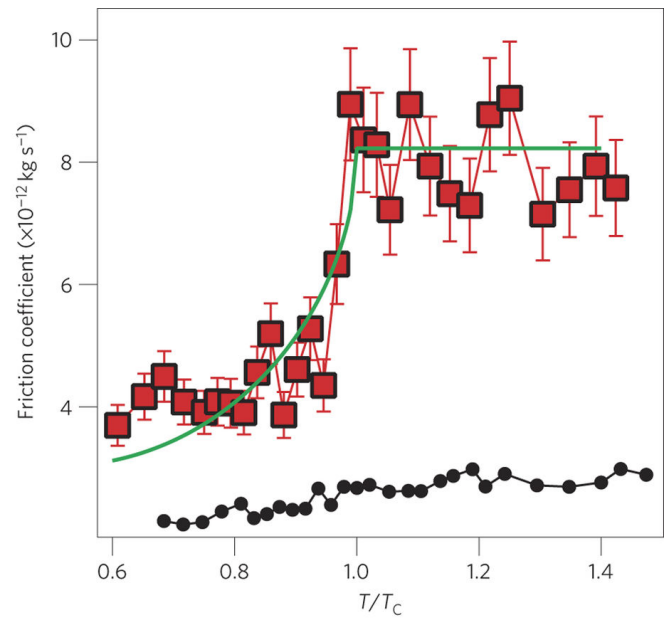

Abbildung 2.13 - Der Reibungskoeffizient (rote Quadrate) von Niob nimmt unterhalb der supraleitenden Temperatur $T_{C}$ stark ab. Die Daten können dabei gut mit der BSC-Theorie [Bardeen57] (grüne Linie) erklärt werden. Die Messung findet in der Nähe der Oberfläche ohne direkten Kontakt statt. Zum Vergleich sind Reibungsmessungen im großen Abstand durchgeführt worden (schwarze Punkte). Mit freundlicher Genehmigung aus [Kisiel11].

Plötzliche elektronische Änderungen eines Systems sind für Reibungsmessungen daher sehr interessant. Kim et al. [Kim16] untersuchten Reibung auf einer Vanadiumdioxidprobe, die einen stark ausgeprägten Metall-Isolator-Übergang in der Nähe von $T_{\mathrm{MI}} \approx 75^{\circ} \mathrm{C}$ zeigt. Auch hier konnte eine starke Änderung gefunden werden. Die metallische Phase zeigt dabei eine höhere Reibung als die isolierende. Abbildung 2.14 zeigt die gemessene Reibungskraft in Abhängigkeit von der Normalkraft während des Heizens a) und des Abkühlens b). Über Widerstandsmessungen können metallische Phasen erkannt werden. Sie sind hier mit offenen Symbolen dargestellt und zeigen eine größere Steigung, was einem größerem Reibungskoeffizienten entspricht. Abbildung 2.14 c) zeigt den Reibungskoeffizienten in Abhängigkeit von der Temperatur und man erkennt den höheren Reibungskoeffizienten auf der metallischen Phase. Kim et al. erklären ihre Ergebnisse mit der Idee von Qi et al. Sie vermuten, dass sich gefangene Ladungen in eine Fremdphase aus $\mathrm{V}_{2} \mathrm{O}_{5}$ auf der Oberfläche der Probe ansammeln. 

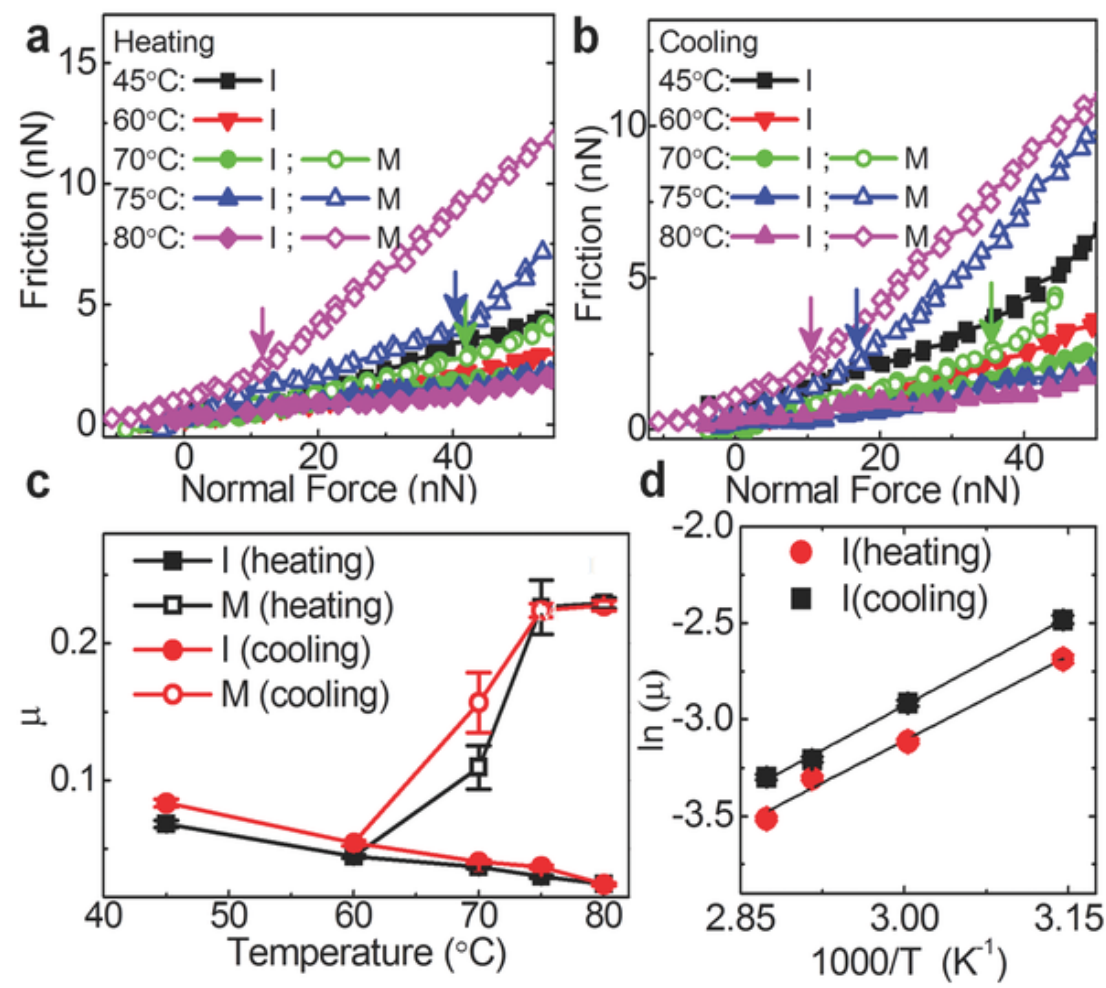

Abbildung 2.14 - Reibungsmessungen an $\mathrm{VO}_{2}$ während Heiz- (a) und Kühlprozess (b). Der Reibungskoeffizient wird aus der Steigung ermittelt. Im metallischen Zustand wird nur die Steigung oberhalb der Pfeile berücksichtigt. Der mittels C-AFM gemessene Widerstand zeigt, ob es sich um eine isolierende (I, volle Zeichen) oder metallische ( $M$, offene Zeichen) Phase handelt. c) Der Reibungskoeffizient ist im metallischen Zustand deutlich erhöht. d) Im isolierenden Zustand nimmt er gemäß Arrhenius-Gleichung 2.11 ab. Mit freundlicher Genehmigung aus [Kim16]. 



\section{Methoden der Mikroskopie - SEHEN, FÜHLEn, Hören}

Anknüpfend an das vorige Kapitel über die theoretischen Arbeiten zur Reibung auf der Nanoskala soll im folgenden Kapitel vor allem die verwendete Messmethode zur Untersuchung der Reibung dargestellt werden. Wie schon erwähnt ist es dabei notwendig, punktförmige Einzelkontakte zu simulieren, um die grundlegenden physikalischen Effekte zu untersuchen. Daher werden vor allem das verwendete Rasterkraftmikroskop und der Reibungskraft-Messmodus erläutert. Da im Rahmen dieser Arbeit eine neue Messmethode auf Basis der Ultraschallmikroskopie entwickelt worden ist, um Reibung zu bestimmen, werden auch Grundlagen zur Ultraschallmikroskopie erklärt.

\subsection{ElektronenMikroskop}

Etymologisch betrachtet beschäftigt sich die Mikroskopie damit, etwas Kleines (alt-

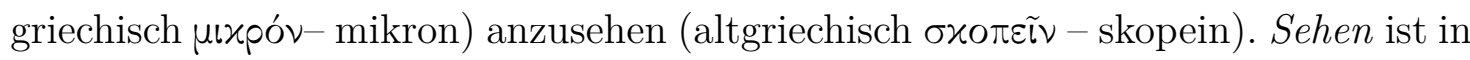
diesem Fall wörtlich zu nehmen, denn die bekannteste und gleichzeitig auch älteste Möglichkeit, unsichtbar kleine Dinge sichtbar zu machen, ist die Lichtmikroskopie, die Ende des 16. Jahrhunderts entwickelt worden ist. Gemäß Abbe-Kriterium ist das Aufösungsvermögen proportional zur Wellenlänge $\lambda$ der elektromagnetischen Welle, so dass moderne Lichtmikroskope mit Immersionsflüssigkeiten Auflösungsvermögen von etwa 0,2 $\mathrm{mm}$ erreichen können ${ }^{1}$. Heute werden daher Röntgen- oder Elektronenmikroskope verwendet, um die Auflösung deutlich zu vergrößern.

In der vorliegenden Arbeit wird ein kommerzielles Rasterelektronenmikroskop (REM) LEO Supra 35 der Firma Zeiss genutzt, um die verwendeten Messspitzen zu untersuchen. Spitzenveränderungen können abgeschätzt werden, indem Bilder der Messspitzen vor und nach der Messung aufgenommen und diese miteinander

\footnotetext{
${ }^{1}$ Es ist möglich, das Abbe-Kriterium zu umgehen. Stefan Hell bekam beispielsweise für die Entwicklung der superauflösenden Fluoreszenzmikroskopie 2014 den Nobelpreis [Hell94, Klar99].
} 
verglichen werden. Das verwendete REM nutzt aus einem Glühdraht emittierte Elektronen, die mit elektrischen Feldern auf etwa $5 \mathrm{keV}$ beschleunigt werden. Durch magnetische Felder wird ein feiner Elektronenstrahl erzeugt, dessen Fokus auf der Oberfläche der Probe liegt. Aus dieser werden Sekundärelektronen mit einigen eV aus der k-Schale des Oberflächenmaterials der Probe ausgelöst und anschließend vom Gerät durch einen Inlens-Detektor gezählt. Aufgrund der niedrigen Energien dieser Elektronen werden nur die der obersten Atomlagen ins Vakuum abgegeben. Durch topographische Effekte wie Stufen oder Kanten, aber auch durch Materialänderungen werden unterschiedlich viele Elektronen ausgelöst bzw. detektiert. Rastert man nun über die Probenoberfläche, entsteht ein dreidimensional wirkendes Bild, das topografische und materialspezifische Kontraste enthält. Abbildung 3.1 zeigt eine der verwendeten Spitzen.

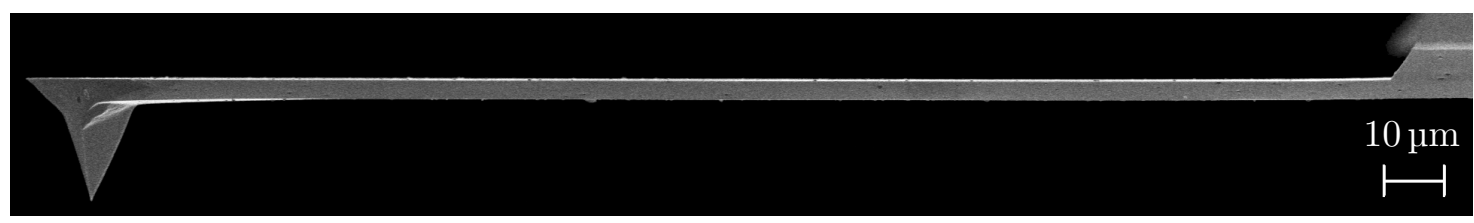

Abbildung 3.1 - REM-Aufnahme einer verwendeten Blattfeder mit Messspitze mit einer Gesamtlänge von etwa $230 \mu \mathrm{m}$.

\subsection{RASTERKRAFTMIKROSKOP}

Eine weitere Form der Mikroskopie ist die Rastersondenmikroskopie, zu der auch das Rasterkraftmikroskop gehört. Dabei werden in Abhängigkeit vom Ort Wechselwirkungen zwischen einer Sonde und einer Probenoberfläche gemessen und anschließend zu einem Bild zusammengesetzt. Im Vergleich zur Lichtmikroskopie wird also nicht mehr optisch hingesehen, sondern mit einem "Tastfinger" gefühlt.

Der Ingenieur Gustav Schmaltz entwickelte bereits 1929 - mit verblüffender Ähnlichkeit zum heutigen Rasterkraftmikroskop - den ersten Profilometer [Schmaltz29]. Er verwendete eine Nadel als Sonde, die über eine Oberfläche - in seinem Fall Holz - gezogen wurde. Ein Spiegel, der an der Spitze angebracht war, reflektierte Licht. Schmaltz maß die Auslenkung des Lichts während des Scanvorgangs und bekam so ein Signal proportional zum Oberflächenprofil.

Entscheidend für die Entwicklung des Rasterkraftmikroskops war die vorhergehende Erfindung des Rastertunnelmikroskops [Binnig83], das im ersten Rasterkraftmikroskop Verwendung fand. Binnig und Rohrer nutzten dafür den Tunneleffekt, 
um Oberflächen im Realraum darzustellen. Auch wenn die technische Umsetzung schwierig war, ist das Prinzip des Rastertunnelmikroskops einfach. Bisherige Tunnelexperimente untersuchen Metall-Isolator-Metall-Schichtpakete, wobei ein Tunnelstrom über den Isolator fließt. Ein Rastertunnelmikroskop besteht aus einer Metallspitze, die mittels Piezostellelementen über eine Metalloberfläche bewegt wird. Der Isolator ist in diesem Fall das Vakuum, in dem die Messung durchgeführt wird. Da der Tunnelstrom eine Exponentialfunktion des Abstandes ist, ist diese Messung sehr sensitiv auf den Abstand der Spitze zur Oberfläche der Probe. Bereits in ihrer ersten Veröffentlichung zum Rastertunnelmikroskop konnten Binnig und Rohrer monoatomare Stufen mit der Stufenhöhe von $3 \AA$ einer Siliziumoberfläche zeigen [Binnig83]. Binnig und Rohrer erhielten 1986 für ihre Konstruktion des Rastertunnelmikroskops den Nobelpreis für Physik.

Da ein Tunnelstrom metallische Elektroden voraussetzt, ist es nicht möglich, Oberflächen isolierender Materialien mit dem Rastertunnelmikroskop zu vermessen. Dieses Problem löste Binnig zusammen mit Calvin F. Quate und Christoph Gerber 1985, indem sie Ideen - wie zum Beispiel der Profilometer von Schmaltz - mit dem Rastertunnelmikroskop verbanden. Das Resultat war das Rasterkraftmikroskop [Binnig86] - oder auch Atomkraftmikroskop (engl. Atomic Force Microscope, $\mathrm{AFM})^{2}$.

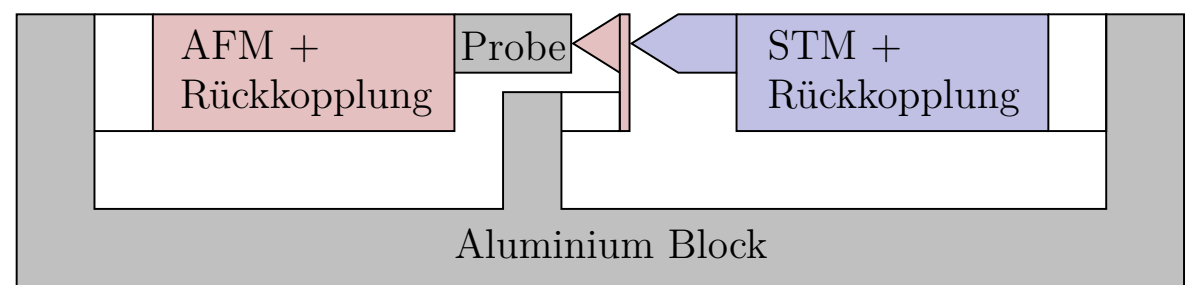

Abbildung 3.2 - Das erste Rasterkraftmikroskop nutzt ein Rastertunnelmikroskop (STM), um die Auslenkung einer Tastspitze (AFM) zu messen. Nach [Binnig86].

Abbildung 3.2 zeigt ebendiese Kombination aus mechanischer Tastspitze und Rastertunnelmikroskop. Die Komplexität des Aufbaus - nämlich die Kombination aus STM und AFM und der zugehörigen Rückkopplungen - führte dazu, dass weitere Möglichkeiten gesucht wurden, um die Auslenkung der Tastspitze zu messen. Andere Ideen, wie zum Beispiel der Einsatz von Interferometern [Martin87b, McClelland87], wurden erfolgreich umgesetzt. Heutige kommerzielle Rasterkraftmi-

\footnotetext{
${ }^{2}$ Auch wenn der Begriff Atomkraftmikroskop deutlich macht, dass es sich um ein Verfahren mit atomarer Auflösung handelt, wird im Folgenden der - im deutschen üblichere - Begriff Rasterkraftmikroskop verwendet.
} 
kroskope - wie auch das für diese Arbeit verwendete - basieren jedoch auf der Idee von Gerhard Meyer und Nabil M. Amer 1988 [Meyer88], die Laserlicht und einen Positionsdetektor einsetzten.

Die Messung des AFM basiert auf der Messung der Verbiegung einer Messspitze, was als Pendant zur Messung des Tunnelstroms des STM verstanden werden kann. Diese Verbiegung entsteht durch Wechselwirkungen zwischen Messspitze und Probenoberfläche, die aus attraktiven und repulsiven Kräften besteht. Zur Vermessung der Verbiegung wird ein - in dieser Arbeit Helium-Neon- - Laserstrahl auf der Rückseite einer Blattfeder reflektiert und auf einen Vier-Quadrant-Photodetektor gelenkt. Am vorderen Ende der Blattfeder befindet sich die Messspitze. Wird nun die zu untersuchende Probe beziehungsweise der Messaufbau bestehend aus Photodetektor, Laser und Blattfeder bewegt, verbiegt sich die Blattfeder - je nach Oberflächenstruktur - aus seiner Ruhelage. Dadurch ändert sich die Position des reflektierten Laserpunktes auf dem Photodetektor. Dieser wiederum besteht aus vier Quadranten, die je nach Lichtintensität eine Spannung ausgeben. Der Vorteil der vier Quadranten ist, dass gleichzeitig vertikale und laterale Auslenkungen aufgenommen werden können. Um hohe Auflösungen zu erreichen, muss es möglich sein, das Bewegen der Spitze sehr genau zu steuern und zu kontrollieren. Dazu werden Piezoelemente mit subatomarer Genauigkeit verwendet, die sich durch Anlegen einer elektrischen Spannung verbiegen. Damit die Spitze in alle drei Raumrichtung bewegt werden kann, verwendet das AFM drei dieser Piezoelemente, die der Raumrichtung nach $x$-, $y$ - oder $z$-Piezo genannt werden. ${ }^{3}$

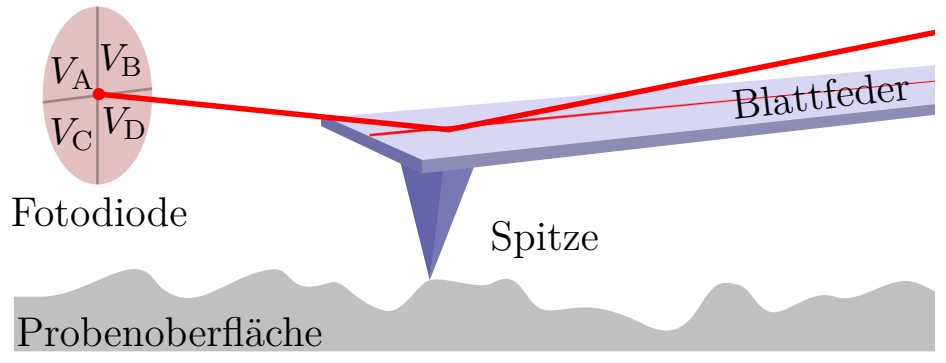

Abbildung 3.3 - Die Topographie einer Oberfläche wird über die Verbiegung der Blattfeder gemessen, indem die Reflexion eines Laserstrahls mit einer Fotodiode gemessen wird.

Die ursprüngliche Idee des Rasterkraftmikroskops, die Oberfläche eines Isolators darzustellen, ist am einfachsten umzusetzen, wenn die Probenoberfläche parallel zur Blattfeder bewegt wird. Dadurch wird die Blattfeder nur vertikal ausgelenkt. Das vertikale Signal $\left(V_{\mathrm{A}}+V_{\mathrm{B}}-V_{\mathrm{C}}+V_{\mathrm{D}}\right.$, siehe Abb. 3.3) ist in diesem Fall proportional zur Topographie der Probe.

\footnotetext{
${ }^{3}$ Meist wird zur Bewegung in der Ebene nur ein Röhrenpiezo verwendet, der gleichzeitig in $x$ und $y$-Richtung bewegt werden kann.
} 


\subsubsection{Kraft-ABstandS-KuRVE}

Im Folgenden werden nur für diese Arbeit wichtige Vorgänge dargestellt, die während der Annäherung von Spitze zur Oberfläche bzw. beim Wegfahren auftreten. Eine umfangreiche Ausführung zum Thema der Kraft-Abstands-Kurven eines AFM liefert zum Beispiel Capella und Dietler [Cappella99].

Die erwähnten repulsiven und attraktiven Kräfte sollen nun etwas genauer betrachtet werden. Da diese Kräfte unterschiedlich vom Abstand abhängen, bietet es sich an, sich die Spitze in Abhängigkeit vom Abstand $D$ zur Probenoberfläche anzuschauen. Experimentell wird dies anhand so genannter Kraft-Abstands-Kurven vollzogen - dabei wird der z-Piezo verfahren und die Verbiegung der Blattfeder gemessen. Man muss hier beachten, dass durch Veränderung der z-Position nicht der Abstand $D$, sondern nur die Verschiebung des $z$-Piezos gemessen wird, so dass man eigentlich von Kraft-Verschiebungs-Kurven sprechen müsste [Cappella99]. Der Abstand $D$ ist nämlich ein Resultat der wirkenden Kräfte an der jeweiligen Position $z$ der Spitze. Dies ist anhand Abbildung 3.4 einfach zu verstehen: Aufgrund der wechselwirkenden Kräfte kann die Spitze aus ihrer Ruhelage um $\sigma_{\mathrm{C}}$ ausgelenkt werden. Außerdem ist es möglich, dass auch die Oberfläche um $\sigma_{\mathrm{s}}$ ausgelenkt wird. Die Distanz $D$ zwischen Spitze und Oberfläche ist daher gegeben durch $D=z-\sigma_{\mathrm{C}}-\sigma_{\mathrm{s}}$.

\section{Abbildung 3.4 - Wird die Spitze mittels z-Piezo an die Probenoberfläche angenähert, wird die Spitze um $\sigma_{C}$ und die Probe um $\sigma_{s}$ ausgelenkt. Die tatsächliche Distanz zwischen Probe und Spitze ist dann $D=z-\sigma_{C}-\sigma_{s}$. Nach [Hao91]}

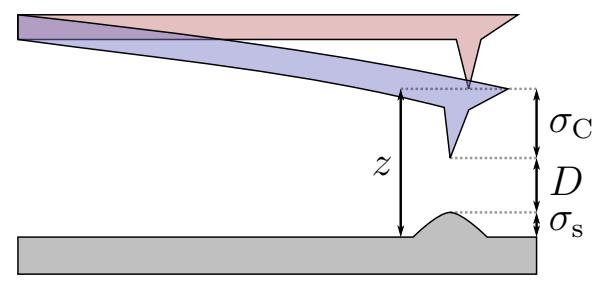

Allgemein kann angenommen werden, dass zwischen Spitze und Oberfläche Wechselwirkungen herrschen, die durch ein Potential $V(D)$ beschrieben werden können, so dass eine Kraft $F(D)$ wirkt. Außerdem ist die Spitze an einer Blattfeder befestigt, die gemäß Hook'schem Gesetz eine Rückstellkraft von $F=-k_{\mathrm{C}} \sigma_{\mathrm{C}}$ ausübt ${ }^{4}$. Wirken bei größeren Distanzen zum Beispiel langreichweitige Van-der-Waals-Kräfte, führen diese dazu, dass die Spitze in Richtung Oberfläche - entgegen der Rückstellkraft - gezogen wird. Dadurch verkleinert sich die Distanz zwischen Spitze und Oberfläche weiter, wodurch sich wiederum größere Van-der-Waals-Kräfte einstellen, so dass die Spitze der Oberfläche noch näher kommt. Das geschieht so lange, bis sich die Beträge der attraktiven Van-der-Waals-Kraft und der Rückstellkraft gleichen.

\footnotetext{
${ }^{4}$ In der vorliegen Arbeit werden nur rechtwinklige Blattfedern verwendet, so dass die Federkonstante analytisch mittels Formel 3.5 berechnet werden kann.
} 
a) Lennard-Jones-Kraft

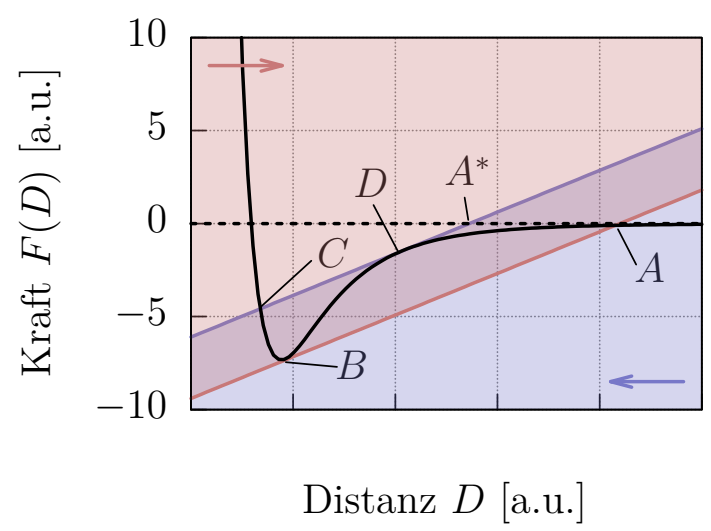

b) Kraft-Abstands-Kurve

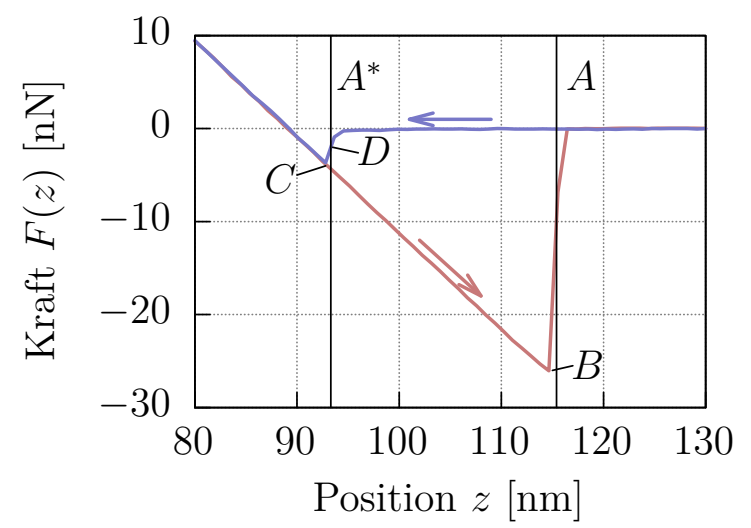

Abbildung 3.5 - Ein nichtlineares Lennard-Jones-Potential führt zu linearen KraftAbstands-Kurven. a) Der Schnittpunkt einer Geraden mit der Steigung der Federkonstanten $k$ und der Lennard-Jones-Kraft entspricht einem Kräftegleichgewicht zwischen Rückstellkraft und Lennard-Jones-Kraft. Die gemessene z-Position der Blattfeder ist in diesem Fall der Schnittpunkt der Geraden mit der $x$-Achse. Kommt man von großen Distanzen $D$ und nähert sich der Probe an, gibt es ab dem Punkt $A$ mindestens zwei Schnittpunkte. In Position $A^{*}$ ändert sich dies und die Spitze springt auf den Punkt C. b) In der Kraft-Abstands-Kurve bleibt die Position $z$ dieselbe, die Auslenkung der Feder und damit die Kraft $F$ ändert sich aber sprunghaft. Auf dem Rückweg (vom roten in den blauen Bereich) geschieht dieser Sprung erst an Position A, da bis zu Punkt $B$ immer ein stabiles Gleichgewicht herrscht.

Abbildung 3.5 a) löst diese Situation grafisch. Eine Annäherung an eine Oberfläche durch Verfahren mittels z-Piezo mit konstanter Geschwindigkeit entspricht in Abbildung 3.5 a) einer Bewegung auf der gestrichelten Geraden mit konstanter Geschwindigkeit in Richtung kleinerer Distanzen. Ein Auslenken der Blattfeder aus einer bestimmten Position $z$ resultiert in einer Rückstellkraft, so dass dies als eine Gerade dargestellt werden kann, deren Schnittpunkt mit der gestrichelten Geraden die Position $z$ angibt und deren Steigung der Federkonstanten $k_{\mathrm{C}}$ entspricht. Befindet man sich zum Beispiel fest an Position $A^{*}$, zeigt die blaue Gerade die Kraft, die benötigt wird, um die Distanz - und damit die Verbiegung der Blattfeder - zu verändern.

Simuliert man nun zusätzliche Wechselwirkungen durch ein einfaches LennardJones-Potential $V=C_{1} / D^{12}-C_{2} / D^{6}$ (schwarze durchgezogene Linie), erfährt die Spitze an Position $A^{*}$ zusätzliche attraktive Kräfte, die die Distanz verringern, bis Rückstellkraft und Lennard-Jones-Kraft den gleichen Betrag haben, also Punkt $D$. 
Fährt man nun ein minimales Stück näher an die Oberfläche, verkleinert sich die Distanz sprunghaft, da es aufgrund der Form der Lennard-Jones-Kraft keinen anderen Schnittpunkt mehr gibt: Die Spitze springt an die Oberfläche zum Punkt $C$, man spricht vom Snap-On-Punkt. Abbildung 3.5 b) zeigt eine Messung einer Kraft-Abstands-Kurve. Die blaue Kurve beschreibt die Annäherung. An Position $A^{*}$ ist die plötzliche Änderung zu erkennen. Fährt man weiter in Richtung Oberfläche, erfährt die Spitze irgendwann die kurzreichweitigen repulsiven Pauli-Kräfte und die Spitze wird nach oben verbogen. Die Distanz bleibt in etwa konstant, die Kraft ändert sich näherungsweise linear und die Position wird weiter mit konstanter Geschwindigkeit verändert. Dadurch ergibt sich in Abbildung 3.5 b) eine lineare Beziehung zwischen Kraft und Position unterhalb von Punkt $C$.

Ändert man nun die Richtung und löst sich so wieder von der Oberfläche, wird wieder Punkt $C$ erreicht. Da sich im Bereich zwischen der Position $A^{*}$ und $A$ aber drei Schnittpunkte ergeben, wobei der mittlere metastabil ist, bleibt die Spitze an der Oberfläche. Erst wenn Punkt $A$, also die rote Gerade, erreicht ist, gibt es nur noch zwei stabile Lösungen. Wie im Hinweg springt die Spitze, diesmal jedoch aus Punkt $B$ auf Punkt $A$, also von der Oberfläche weg, und man spricht vom SnapOff-Punkt. In der Rückrichtung der Messung - beschrieben durch die rote Kurve in Abbildung 3.5 - ergibt sich also wieder ein Sprung, der allerdings größer ist als der Snap-On-Sprung.

\subsubsection{ReIBUngsmikroskopie}

Wird die Probenoberfläche rechtwinklig zur Blattfeder bewegt, führen lateral wirkende Kräfte zu einer Verdrillung der Blattfeder, die über das Lateralsignal $\left(V_{\mathrm{A}}+\right.$ $V_{\mathrm{C}}-V_{\mathrm{B}}+V_{\mathrm{D}}$, siehe Abb. 3.3) gemessen werden kann. In der Literatur wird neben Reibungskraftmikroskopie $(F F M)^{5}$ daher auch der Name Lateralkraftmikroskopie $(\mathrm{LFM})^{6}$ verwendet. Die lateralen Kräfte können durch Reibung entstehen, aber auch topographischen Ursprungs sein. So würden beispielsweise auch Stufen dazu führen, dass beim Überwinden eine größere Torsion der Blattfeder gemessen wird. Um die Reibung zu untersuchen, müssen zunächst derartige topographische Effekte eliminiert werden. Dies wird erreicht, indem sogenannte Reibungshysteresen aufgenommen werden.

Abbildung 3.6 skizziert eine einzelne Reibungshysterese. Dabei wird zunächst der Torsionswinkel $\theta$ der Blattfeder in Hinrichtung aufgezeichnet und anschließend

\footnotetext{
${ }^{5}$ engl. Friction Force Microscopy

${ }^{6}$ engl. Lateral Force Microscopy
} 
in Rückrichtung. Setzt man die Auslenkung in Ruhelage auf $\theta=0$, wird in Hinrichtung eine positive und in Rückrichtung eine negative Spannung gemessen ${ }^{7}$, was einer Verdrillung nach links bzw. rechts entspricht. Trifft die Messspitze nun auf eine Erhöhung desselben Materials, bleibt zwar die Reibungskraft gleich, die Blattfeder wird aber trotzdem stärker ausgelenkt, um die Stufe zu überwinden. Auf dem Rückweg gleitet die Spitze aber dieselbe Stufe hinab, wodurch die Auslenkung verringert wird. Ein Signal proportional zur Reibung wird also gewonnen, indem man die Differenz der Auslenkungen von Hin- und Rückrichtung betrachtet. Um nun darzustellen, welchen Einfluss eine höhere Reibung auf eine Reibungshysterese hat, zeigt Abbildung 3.6 neben der Topographie auch einen Bereich mit höher Reibung (in grau dargestellt). Bewegt man sich auf dem Hinweg von (A) nach (B), kommt es trotz gleichbleibenden Anstiegs aufgrund der größeren Reibung zu einer stärkeren Verdrillung der Blattfeder im Bereich (B). Der Mittelwert der Spannungen $\left(\theta_{\text {Hinrichtung }}+\theta_{\text {Rückrichtung }}\right) / 2$ gibt schließlich ein Signal, das proportional zur Lateralkraft ist [Bennewitz15]. Die schraffierte Fläche der Reibungshysterese entspricht dabei der dissipierten Energie.

\subsubsection{Weitere Messmodi}

Auf Basis des Rasterkraftmikroskops sind weitere Messmodi entwickelt worden. Sie sollen hier kurz erwähnt werden, da sie teilweise in der Literatur zur Tribologie verwendet und somit in der Diskussion erwähnt werden. Da die vorliegende Arbeit folgende Messmodi nicht nutzt, wird auf eine detaillierte Erklärung verzichtet und auf die jeweilige Literatur verwiesen.

Der Nicht-Kontakt-Modus (engl. non-contact mode) ist ein dynamischer Messmodus des AFM, bei dem die Probe nicht berührt wird. Dynamisch, da die Blattfeder zur Schwingung in der Resonanzfrequenz mit Amplituden $<10 \mathrm{~nm}$ angeregt wird. Bringt man die Spitze nun in den Bereich der langreichweitigen van-der-Waals-Kräfte, ändert sich die Frequenz, so dass sie ein Maß für die Wechselwirkungen mit der Probe wird. Typischerweise wird der NichtKontakt-Modus im Vakuum verwendet, da dort aufgrund des fehlenden Wasserfilms weniger Adhäsionskräfte wirken und die Spitze nicht an die Probe springt. In diesem Modus ist die höchste Auflösung eines AFM möglich. [Martin87b, Morita15]

\footnotetext{
${ }^{7} \mathrm{Um} \theta=0$ einzustellen, wird das Spannungssignal vor der Messung exakt auf $0 \mathrm{~V}$ gestellt. Ein Abweichen von $0 \mathrm{~V}$ kann aber auch nachträglich herausgerechnet werden.
} 


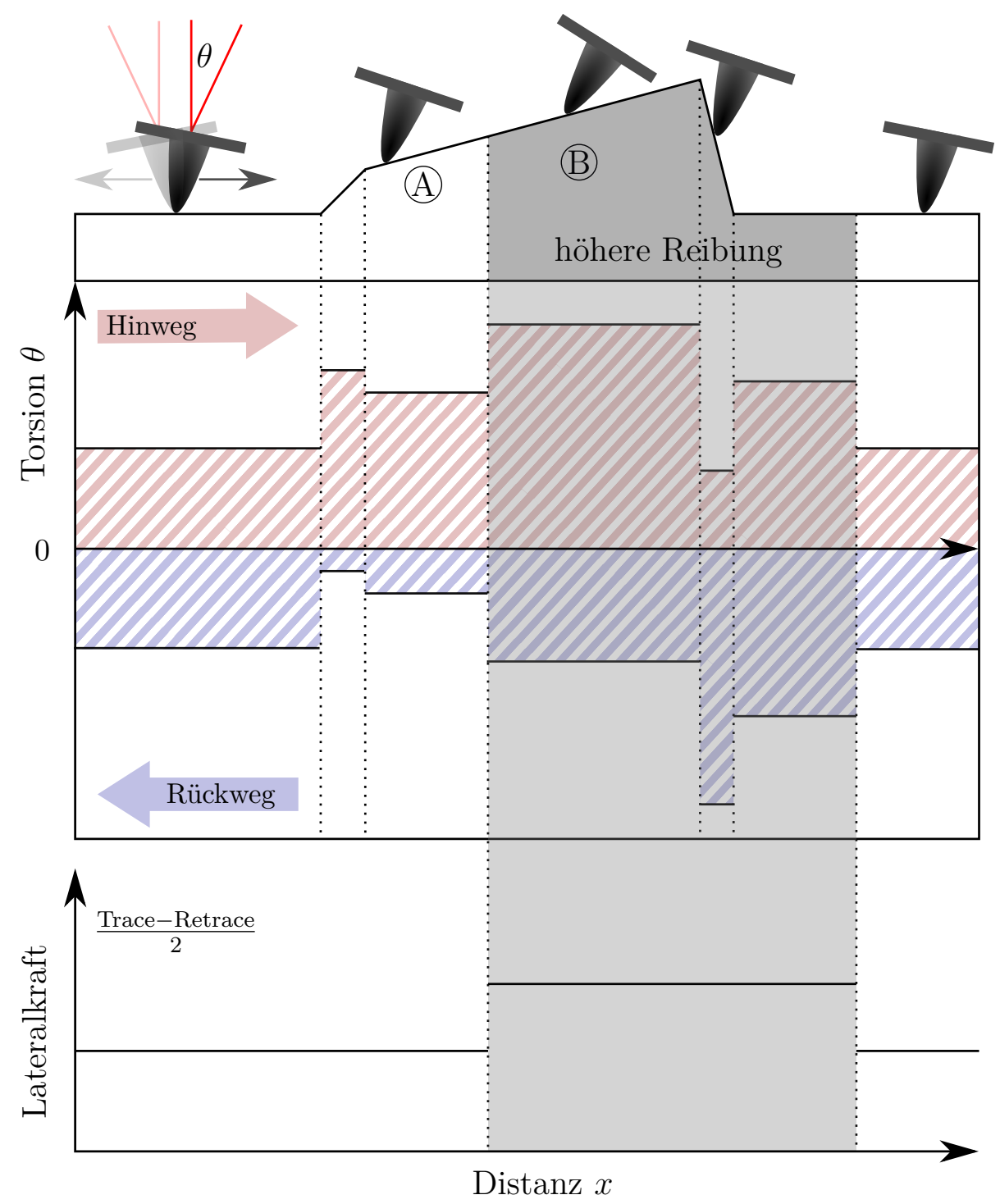

Abbildung 3.6 - Eine Reibungshysterese wird aufgenommen, indem die Torsion $\theta$ einer Blattfeder in Hin- und Rückrichtung aufgenommen wird. Die schraffierte Fläche entspricht dabei der dissipierten Energie. Um topographische Effekte zu eliminieren, wird die Differenz der gemessenen Torsionen beider Richtungen gebildet. Dadurch ergibt sich ein Signal, das proportional zur Lateralkraft ist. 
| Der intermittierende Modus (engl. tapping mode) ist ähnlich zum NichtKontakt-Modus, kann aber unter Normalbedingungen verwendet werden. Auch hier wird die Blattfeder zu Schwingungen angeregt. Allerdings berührt die Spitze in jedem Zyklus die Probe, so dass hier größere Schwingungsamplituden nötig sind, um sie wieder von der Probenoberfläche zu trennen. [Zhong93]

Die Magnetkraftmikroskopie (engl. magnetic force microscopy, MFM) dient der Abbildung lokaler magnetischer Feldstärken. Dabei ist die Messspitze zusätzlich mit einem magnetischen Material beschichtet worden, das auf magnetische Feldstärken, die aus der Probe zeigen, mit einer zusätzlichen Auslenkung reagiert. Vergleicht man das gewonnene Signal mit dem Höhenprofil der Probe, kann man auf das magnetische Profil zurückschließen. [Martin87a]

Die Strom-Spannungs-Mikroskopie (engl. conductive atomic force microscopy, C-AFM) dient unter anderem der Darstellung der Leitfähigkeit in Abhängigkeit vom Ort auf der Probe. Dabei wird eine leitfähige - zum Beispiel mit Platin beschichtete - Spitze eingesetzt. Dadurch, dass eine Spannung zwischen Probe und Messspitze angelegt wird, kann so ein Strom fließen, der zusätzlich zum Höhenprofil Information zur Leitfähigkeit gibt. [Sugawara92, Murrel193]

\subsection{ULtraschalLMikROSKOPIE}

Bereits in den 1970er-Jahren ist von R. A. Lemons und C. F. Quate mit der Rasterultraschallmikroskopie [Lemons74] (engl. scanning acoustic microscope, SAM) ein neues, zerstörungsfreies bildgebendes Verfahren entwickelt worden. Das SAM nutzt akustische Wellen hoher Frequenzen und hört sozusagen in die Probe hinein. Diese akustischen Wellen werden dazu auf eine Probe fokussiert. Die gestreuten Ultraschallwellen werden anschließend detektiert. Das gemessene Signal ist dabei von der akustischen Impedanz $\rho \nu$ der Probe abhängig [Hirsekorn95]. Änderungen der Dichte $\rho$ und/oder der Schallgeschwindigkeit $\nu$ - und damit auch der elastischen Konstanten - führen also zum Kontrast in der Messung. Das heißt, dass diese Messung vor allem auf Änderungen des Materials oder Defekte wie Risse, Hohlräume oder Einschlüsse im Material reagiert, da an solchen Stellen das Ultraschallsignal reflektiert wird. Mit Laufzeitmessungen kann so auch ein Tiefenprofil der Probe erstellt werden. Da auch in der Ultraschallmikroskopie das Abbe-Kriterium gilt, ist eine atomare Auflösung nicht ohne Weiteres möglich. 


\subsubsection{ULTRASCHALLKRAFTMIKROSKOPIE}

Um die Vorteile der Ultraschallmikroskopie dennoch mit atomarer Auflösung zu nutzen, sind verschieden Kombinationen aus Rasterkraftmikroskop und Ultraschallmikroskop entwickelt worden. Beispiele dieser Kombinationen sind das Ultraschallkraftmikroskop $^{8}$ (UFM), das akustische Rasterkraftmikroskop ${ }^{9}$ (SAFM), das Mikrodeformations-Rastermikroskop ${ }^{10}$ (SMM) und die in dieser Arbeit verwendeten Methoden: das akustische Atomkraftmikroskop ${ }^{11}$ (AFAM) und das Ultraschallkraftmikroskop $^{12}$ (UAFM). Der Vorteil zur Ultraschallmikroskopie ist nun, dass nicht sämtliche gestreute Ultraschallwellen detektiert werden, sondern dass lokal mit der Messspitze des AFM Ultraschall detektiert wird. Daher bestimmt nun die Kontaktfläche zwischen Messspitze und Probe die Auflösung.

\subsubsection{AFAM}

Wie bereits angedeutet wurde, kombiniert das AFAM das AFM mit Anregungen durch Ultraschall. Dazu wird ein Schallwandler verwendet, der mittels piezoelektrischer

Materialien elektrische Spannungen in Materialverformungen umwandelt. Durch sinusförmige Variationen der elektrischen Spannung mit hohen Frequenzen können so Ultraschallwellen erzeugt werden. Da der piezoelektrische Effekt durch die Verschiebung der Ladungsschwerpunkte der Elementarzellen des Materials ausgelöst wird, kann er auch umgekehrt verwendet werden. Daher kann ein Schallwandler zum Messen von Schallwellen über die entstehende Spannung am Material eingesetzt und so als Prüf-

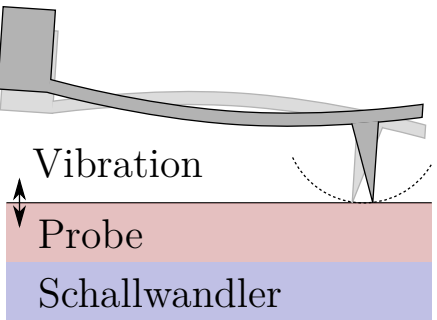

Abbildung 3.7 - Skizze des AFAM. Ein Schallwandler regt die Blattfeder zu Schwingungen an kopf verwendet werden.

Im AFAM wird ein solcher Schallwandler mit einem Kopplungsmedium - hier meist Honig - an der Unterseite der Probe angebracht. Dadurch können Biegeund/oder Torsionsmoden der Blattfeder, die im Kontakt mit der Probe ist, angeregt werden. Frequenzen und Amplituden dieser Schwingungen können dann über den

\footnotetext{
${ }^{8}$ engl. Ultrasonic Force Microscope [Kolosov93]

${ }^{9}$ engl. Scanning Acoustic Force Microscope [Hesjedal01]

${ }^{10}$ engl. Scanning Microdeformation Microscope [Cretin93]

${ }^{11}$ engl. Atomic Force Acoustic Microscope [Rabe94]

${ }^{12}$ engl. Ultrasound Atomic Force Microscope [Yamanaka96]
} 
jeweiligen Kanal der Photodiode des AFM ausgelesen werden.

Abbildung 3.7 skizziert den Aufbau eines AFAM. Die Frequenz der ersten Biegeschwingungmode ist höher als die freie Frequenz der Blattfeder - typischerweise etwa zwei bis fünf Mal so hoch. Die verwendeten Blattfedern haben Eigenfrequenzen von etwa 20 bis $200 \mathrm{kHz}$.

\subsubsection{KontakTRESOnANZMOdELLE}

Die Messwerte einer AFAM-Messung sind Frequenz und Amplitude der Blattfeder, die sich im Kontakt mit der Probe befindet. Um daraus materialspezifische Werte zu berechnen, muss zunächst ein Modell des Kontakts aufgestellt und gelöst werden. Dieses Modell kann sehr komplex werden.

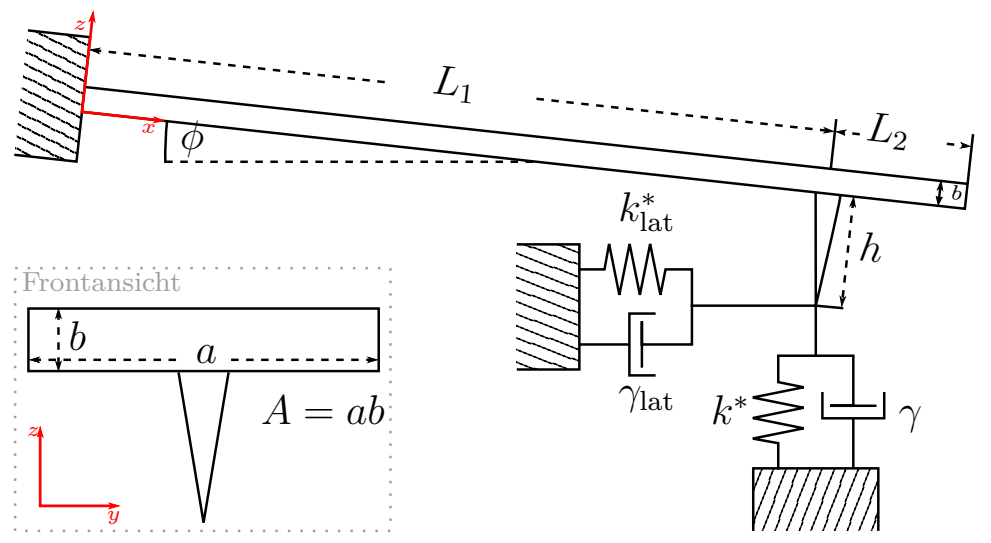

Abbildung 3.8 - Das Kontaktmodell einer Blattfeder im Kontakt mit einer Probe enthält Dämpfungstöpfe und $\mathrm{Fe}$ dern für laterale und vertikale Auslenkungen. Nach [Rabe06].

Abbildung 3.8 zeigt das Modell, das für diese Arbeit verwendet wird. Es besteht aus einer Blattfeder in Form eines Quaders mit der Länge $L=L_{1}+L_{2}$, an dem eine Spitze mit der Höhe $h$ an der Position $x=L_{1}$ angebracht ist. Die Blattfeder steht in einem Winkel $\phi$ zur Probenoberfläche. Der Kontakt mit der Probe wird durch die Kontaktsteifigkeit $k^{*}$ und die Dämpfung $\gamma$ sowie ihren lateralen Varianten $k_{\text {lat }}^{*}$ und $\gamma_{\text {lat }}$ realisiert.

Zur Veranschaulichung wird zunächst ein deutlich einfacheres Modell behandelt. Unter der Annahme, dass die Blattfeder parallel zur Probenoberfläche ist $(\phi=0)$, dass sich die Spitze am vorderen Ende der Blattfeder befindet $\left(L_{2}=0\right)$ und dass nur Normalkräfte wirken $\left(k_{\text {lat }}^{*}=0\right)$, können einfache Randbedingungen aufgestellt werden. Nimmt man weiter an, dass die Amplitude der Blattfeder durch die Funktion $y(x, t)$ beschrieben wird, müssen zunächst an der Stelle $x=0$ Steigung und 
Auslenkung der Blattfeder null sein, da sie fest am Chip befestigt ist:

$$
y(0, t) \stackrel{!}{=} 0 \quad \frac{\partial y(0, t)}{\partial x} \stackrel{!}{=} 0 .
$$

Das andere Ende hingegen muss frei von Momenten und Kräften ${ }^{13}$ sein:

$$
\frac{\partial^{2} y(L, t)}{\partial x^{2}} \stackrel{!}{=} 0 \quad \frac{\partial y^{3}(L, t)}{\partial x^{3}} \stackrel{!}{=} \frac{3 k^{*}}{E I} y
$$

Dabei ist $I=\frac{a b^{3}}{12}$ das Flächenträgheitsmoment eines Rechtecks mit der Höhe $b$ und der Breite $a$. Nach Rabe et al. [Rabe96] findet man mit einer allgemeinen Lösung der Form

$$
\begin{aligned}
y(x)= & A(\cos k x+\cosh k x)+B(\cos k x-\cosh k x) \\
& +C(\sin k x+\sinh k x)+D(\sin k x-\sinh k x)
\end{aligned}
$$

nach Einsetzen in die Randbedingungen (Gl. 3.1 und 3.2) die Beziehung

$$
\frac{k^{*}}{k_{\mathrm{C}}}=\frac{\left(k_{n} L\right)^{3}\left(1+\cos k_{n} L \cosh k_{n} L\right)}{3\left(\sinh k_{n} L \cos k_{n} L-\sin k_{n} L \cosh k_{n} L\right)},
$$

wobei die Federkonstante einer Blattfeder mit Elastizitätsmodul $E$, Höhe $b$, Breite $a$ und Länge $L$ gegeben ist durch

$$
k_{\mathrm{C}}=\frac{E b^{3} a}{4 L^{3}}=\frac{3 E I}{L^{3}} .
$$

Die Herleitung ohne die obigen Vereinfachungen ist prinzipiell ähnlich, aufgrund weiterer Variablen allerdings deutlich komplexer. Unter anderem in den Arbeiten [Rabe06, Kopycinska08, Phani16] wird diese Herleitung ausführlich beschrieben, daher sollen hier nur die Ergebnisse zusammengefasst werden. Die Lösung der Differenzialgleichung fordert die charakteristische Gleichung

\footnotetext{
${ }^{13}$ Da eine Auslenkung $y$ eine Kraft $-k^{*} y$ hervorruft, muss gelten $E I \frac{\partial y^{3}(L, t)}{\partial x^{3}}-k^{*} y=0$
} 


$$
\begin{aligned}
0 & =2 A_{4}+3 \frac{k^{*}}{k_{\mathrm{C}}}\left[3 A_{0} c_{p} \frac{k^{*}}{k_{\mathrm{C}}}\left(\frac{h}{L_{1}}\right)^{2}+A_{1}\left(\cos ^{2} \phi+c_{p} \sin ^{2} \phi\right)\right. \\
& \left.+2 A_{2} \frac{h}{L_{1}}\left(\sin \phi \cos \phi\left(c_{p}-1\right)\right)+A_{3}\left(\frac{h}{L_{1}}\right)^{2}\left(\sin ^{2} \phi+c_{p} \cos ^{2} \phi\right)\right]
\end{aligned}
$$

wobei die Parameter $A_{0}$ bis $A_{4}$ über die Gleichungen 3.7(a-e) definiert sind. Diese wiederum nutzen die Variablen der Gleichungen 3.8(a-d), wobei das Subskript $i$ den Wert 1 oder 2 annehmen kann. Die Variable $c_{p}=k_{\text {lat }}^{*} / k^{*}$ gibt das Verhältnis zwischen lateraler und normaler Kontaktsteifigkeit an.

$$
\begin{aligned}
& A_{0}=a_{1} a_{2} \\
& A_{1}=k_{n} L_{1}\left[a_{2} b_{1}-a_{1} b_{2}\right] \\
& A_{2}=\left(k_{n} L_{1}\right)^{2}\left[a_{2} c_{1}+a_{1} c_{2}\right] \\
& A_{3}=\left(k_{n} L_{1}\right)^{3}\left[a_{2} d_{1}-a_{1} d_{2}\right] \\
& A_{4}=\left(k_{n} L_{1}\right)^{4}\left(1+\cos k_{n} L \cosh k_{n} L\right) \\
& a_{i}=1+(-1)^{i} \cos k_{n} L_{i} \cosh k_{n} L_{i} \\
& b_{i}=\sin k_{n} L_{i} \cosh k_{n} L_{i}-\sinh k_{n} L_{i} \cos k_{n} L_{i} \\
& c_{i}=\sin k_{n} L_{i} \sinh k_{n} L_{i} \\
& d_{i}=\sin k_{n} L_{i} \cosh k_{n} L_{i}+\sinh k_{n} L_{i} \cos k_{n} L_{i}
\end{aligned}
$$

Die Nullstellen der Gleichung 3.6 sind

$$
X=\frac{1}{3}\left(z \pm \sqrt{z^{2}-\frac{2 A_{4} L_{1}^{2}}{A_{0} c_{p} h^{2}}}\right)
$$

wobei hier der Parameter

$$
z=\frac{A_{1}\left(\cos ^{2} \phi+c_{p} \sin ^{2} \phi\right)+2 A_{2} \frac{h}{L_{1}}\left(c_{p}-1\right) \sin \phi \cos \phi+A_{3}\left(\frac{h}{L_{1}}\right)^{2}\left(\sin ^{2} \phi+c_{p} \cos ^{2} \phi\right)}{-2 A_{0} c_{p}\left(\frac{h}{L_{1}}\right)^{2}}
$$


verwendet wird.

Zur Überprüfung kann das einfachere Modell mit der Lösung 3.4 aus Gleichung 3.6 entwickelt werden, wenn man wieder annimmt, dass $k_{\text {lat }}^{*}=0$ und dadurch $c_{p}=0$ und dass $L_{2}=\phi=0$. Dadurch vereinfacht sich Gleichung $3.6 \mathrm{zu}$

$$
\begin{aligned}
0 & =2 A_{4}+3 \frac{k^{*}}{k_{\mathrm{C}}} A_{1} \\
\Rightarrow \frac{k^{*}}{k_{\mathrm{C}}} & =\frac{-2 A_{4}}{3 A_{1}}=\frac{-\left(k_{n} L\right)^{3}\left(1+\cos k_{n} L \cosh k_{n} L\right)}{3\left(\sin k_{n} L \cosh k_{n} L-\sinh k_{n} L \cos k_{n} L\right)},
\end{aligned}
$$

was Gleichung 3.4 entspricht. Weiter ist es damit möglich, den Fall der freien Blattfeder zu berechnen, indem man annimmt, dass in diesem Fall $k^{*}=0$ gilt:

$$
0=1+\cos k_{n, \text { free }} L \cosh k_{n, \text { free }} L .
$$

Die Lösungen der ersten vier Moden sind in Tabelle 3.1 aufgeführt.

\begin{tabular}{|c|c|c|c|c|}
\hline$n$ & 1 & 2 & 3 & 4 \\
\hline$k_{n, \text { free }} L$ & 1,87510 & 4,69409 & 7,85476 & 10,99554 \\
\hline
\end{tabular}

Tabelle 3.1 - Die ersten vier Lösungen der charakteristischen Gleichung 3.12 für die einseitig freie Blattfeder.

Die zur $n$-ten Mode zugehörige Frequenz kann berechnet werden über [Rabe96]

$$
f_{n}=\left(\frac{k_{n} L}{c_{\mathrm{c}}}\right)^{2}
$$

wobei die geometrischen und mechanischen Eigenschaften und die Dichte $\rho$ der Blattfeder über

$$
c_{\mathrm{c}}=L \sqrt[4]{\frac{4 \pi^{2} \rho A}{E I}}
$$

berücksichtigt werden.

Um nun aus den gemessenen Daten elastische und dämpfende Eigenschaften zu bestimmen, muss zunächst der komplexe Wellenvektor der Blattfeder bestimmt werden [Yuya08, Yuya11, Killgore11, Phani16]:

$$
k_{n, \mathrm{cont}}=\frac{R_{n}+i I_{n}}{L},
$$


mit

$$
\begin{aligned}
R_{n} & =k_{n, \text { free }} \sqrt{\frac{f_{n, \text { cont }}}{f_{n, \text { free }}}} \\
I_{n} & =\frac{R_{n}}{4}\left(Q_{n, \text { cont }}^{-1}-Q_{n, \text { free }}^{-1} \frac{f_{n, \text { free }}}{f_{n, \text { cont }}}\right) .
\end{aligned}
$$

Die Gütefaktoren $Q_{n \text {,cont }}$ und $Q_{n \text {,free }}$ sowie die Frequenzen $f_{n, \text { cont }}$ und $f_{n \text {,free }}$ im Kontakt bzw. im freien Zustand können dann durch die gemessenen Resonanzkurven bestimmt werden. Dazu werden Resonanzkurven des freien Zustands und des Kontakts gemessenen und an eine Cauchy-Lorentzfunktion angepasst. Sofern alle Parameter der Blattfeder bestimmt sind, kann Gleichung 3.9 gelöst werden, was schließlich zur normierten Kontaktsteifigkeit [Phani16]

$$
\frac{k^{*}}{k_{\mathrm{C}}}=\operatorname{Re}(X)+i \operatorname{Im}(X)\left(\frac{k_{n, \text { cont }}}{k_{n, \text { free }}}\right)^{2} \frac{f_{n, \text { free }}}{f_{n, \text { cont }}}
$$

führt. Die Dämpfung $\gamma$ aus Abbildung 3.8 ist dabei mit dem Imaginärteil der Kontaktsteifigkeit verknüpft durch

$$
\omega_{n, \mathrm{cont}} \gamma=\operatorname{Im}(X) \frac{3 E I}{L_{1}^{3}} .
$$

Nimmt man an, dass $R_{n} \gg I_{n}$, ist der Realteil der Kontaktsteifigkeit gegeben durch

$$
k_{r}^{*}=\operatorname{Re}(X) k_{\mathrm{C}}
$$

Mit Gleichung 3.5 kann schließlich die lokale Dämpfung [Yuya08, Caron09, Phani16]

$$
Q_{\mathrm{loc}}^{-1}=\frac{E^{\prime \prime}}{E^{\prime}}=\frac{\omega_{n, \mathrm{cont}} \gamma}{k_{r}^{*}}=\frac{\operatorname{Im}(X)}{\operatorname{Re}(X)}\left(\frac{L}{L_{1}}\right)^{3}
$$

bestimmt werden. Dabei ist $E^{\prime \prime}$ der Verlustmodul und $E^{\prime}$ der Speichermodul.

In der Fachliteratur werden meist weitere Parameter eingeführt. Dies ist eher historisch bedingt und bringt weder Vorteile - da trotzdem zunächst Gleichung 3.9 gelöst wird - noch tiefere physikalische Einblicke, da die physikalischen Größen, die von Interesse sind, die lokale Dämpfung und die lokale Kontaktsteifigkeit bleiben. Dennoch sollen zum Vergleich und zum Verständnis der Literatur diese Parameter hier kurz aufgeführt werden. Der Realteil von $X$ aus Gleichung 3.9 wird dabei $\lambda$ 
oder auch $\alpha$ gennant und ist definiert wie in Gleichung 3.20:

$$
\alpha=\lambda=\operatorname{Re}(X)=\frac{k_{r}^{*}}{k_{\mathrm{C}}} .
$$

Der Imaginärteil von $X$ fließt in einen Parameter namens $\beta$ oder auch $\eta$ über

$$
\beta_{n}=\eta_{n}=\frac{\operatorname{Im}(X)}{a_{n}^{2}\left(\frac{L_{1}}{L}\right)^{2}}=\gamma \frac{L_{1}}{\sqrt{9 E I \rho A}}
$$

ein und wird meist als normalisierte Dämpfungskonstante bezeichnet. Dadurch ergibt sich für Gleichung 3.21

$$
\begin{aligned}
& Q_{\text {loc }}^{-1}=\frac{\eta_{n}}{\lambda} \frac{L}{L_{1}} \frac{\omega_{n, \text { cont }}}{\omega_{n, \text { free }}}\left(k_{n, \text { free }} L\right)^{2} \\
& \stackrel{n=1}{=} 3,516 \frac{\eta_{1}}{\lambda} \frac{L}{L_{1}} \frac{\omega_{1, \text { cont }}}{\omega_{1, \text { free }}}
\end{aligned}
$$

Dabei gibt Gleichung 3.25b den Fall für die erste Mode an. 



\section{$4 \mid$ Probensysteme}

Nach den experimentellen Messmethoden der Reibungsmessung sollen nun die Proben vorgestellt werden, an denen Reibung gemessen worden ist. Bei den untersuchten Probensystemen handelt es sich zum einen um das stark korrelierte Übergangsmetallperowskit $\mathrm{La}_{1-x} \mathrm{Sr}_{x} \mathrm{MnO}_{3}$, zum anderen um $\mathrm{Pd}_{77,5} \mathrm{Cu}_{6} \mathrm{Si}_{16,5}$ in amorpher und kristalliner ${ }^{1}$ Form.

Zunächst sollen die physikalischen Eigenschaften beider Systeme vorgestellt werden. Anschließend wird kurz auf die unterschiedlichen Methoden der Probencharaktisierung eingegangen.

\subsection{Manganate}

Ein untersuchtes Probensystem ist $\mathrm{La}_{0,6} \mathrm{Sr}_{0,4} \mathrm{MnO}_{3}$ (LMSO), das zu den Manganaten und damit zu den stark korrelierten Übergangsmetallperowskiten gehört. Nachdem seit 1926 strukturelle Untersuchungen [Goldschmid26, Goldschmid43, Megaw46] an den Manganaten durchgeführt worden waren, waren es Jonker und van Santen, die 1950 elektronische und magnetische [Jonker50, Santen50] Messungen an strontium-, lanthan- oder bariumdotierten $\mathrm{LaMnO}_{3}$ veröffentlichten. Sie fanden eine Erhöhung der Curie-Temperatur im Bereich von 25\% bis 40\% Dotierung und eine Anomalie des Widerstands in deren näherer Umgebung. Jonker und van Santen sahen in diesem System den ersten eindeutigen Nachweis für eine positive Austauschwechselwirkung und vermuteten korrekt, dass diese von indirektem Typ ist.

Bereits diese Arbeiten deuteten an, dass Manganate einen großen Phasenreichtum zeigen. Heute weiß man, dass die chemische Zusammensetzung entscheidend für elektrische und magnetische Eigenschaften ist und dass zum Beispiel externe Felder, Temperatur, Druck oder Licht Phasenübergänge hervorrufen können. So ist

\footnotetext{
${ }^{1}$ Die kristallinen Proben wurden durch Auslagern von Teilen der amorphen Probe bei hohen Temperaturen hergestellt. Daher ist kein Einkristall zu erwarten und man müsste von einer polykristallinen Probe sprechen - darauf wird aber im Folgenden verzichtet.
} 
zum einen Supraleitung gefunden [Bednorz86], zum anderen ist der kolossale Magnetowiderstand [Chahara93, Helmolt93] (engl. Colossal Magnetoresistance, CMR) entdeckt worden, der die elektrische Leitfähigkeit durch ein externes Magnetfeld um mehrere Größenordnungen verändert.

Die folgenden Abschnitte sollen einige Grundlagen der Manganate darstellen. Zunächst wird die Kristallstruktur gezeigt, anhand derer anschließend die elektronische Struktur erklärt wird. Diese wiederum hat über Gitterverzerrungen Einfluss auf den Überlapp von Atomorbitalen, wodurch Austauschwechselwirkungen und damit magnetische Eigenschaften beeinflusst werden. Abschließend werden die gewonnen Erkenntnisse genutzt, um Bereiche der Phasendiagramme zu erklären.

\subsubsection{KristallstruKtuR}

Manganate kristallisieren in verschiedenen Varianten der Struktur von Perowskit ${ }^{2}$. Die allgemeine Summenformel ist $A_{B O}$ (s. Abb. 4.1), wobei der $A$-Platz in der vorliegenden Arbeit durch das divalente Erdalkalimetall Strontium oder das trivalente Übergangsmetall der seltenen Erden Lanthan besetzt wird. Umgeben von einem Sauerstoffoktaeder sitzt auf dem zentralen B-Platz ein Manganion, das je nach umgebender $A$-Platzbesetzung eine Valenz zwischen $\mathrm{Mn}^{3+}$ und $\mathrm{Mn}^{4+}$ hat.

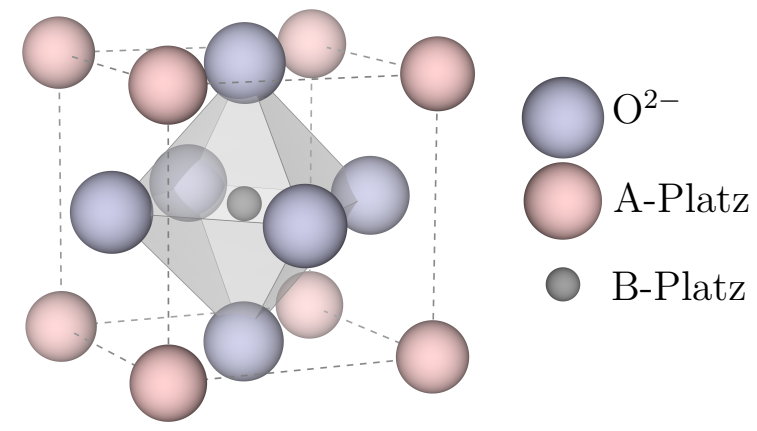

Abbildung 4.1 - Einheitszelle der kubischen Variante einer Perowskitstruktur der Form $\mathrm{ABO}_{3}$. Das B-Platz-Ion (schwarz) ist von sechs Sauerstoffionen (blau) umgeben. Auf den Ecken der Einheitzzelle sitzen die A-PlatzIonen (rot). Erstellt mit VESTA 3 [Momma11].

Abbildung 4.1 zeigt die idealisiert kubische Variante der Perowskitstruktur. Die reale Struktur hängt von den Ionenradien $R_{A / B / O}$ der Kationen ab und kann über den - nach Victor M. Goldschmidt benannten - Goldschmidt'schen Toleranzfaktor [Goldschmid26]

$$
t=\frac{1}{\sqrt{2}} \frac{R_{A}+R_{\mathrm{O}}}{R_{B}+R_{\mathrm{O}}}
$$

\footnotetext{
${ }^{2}$ Der deutsche Mineralologe Gustav Rose beschrieb 1939 zum ersten Mal das Mineral CaTiO 3 und benannte es nach dem russischen Politiker und Mineralogen Lew Alexejewitsch Perowski.
} 
quantifiziert werden. Eine kubische Struktur stellt sich nur für $t=1$ ein. Ein von 1 abweichendes $t$ lässt die Sauerstoffoktaeder verkippen. Im Bereich $0,96<t<1$ liegt die pseudokubische rhomboedrische Struktur ( $\mathrm{R} \overline{3} \mathrm{c}$ ) vor, für $t<0,96$ stellt sich eine orthorhombische Struktur (Pnma) ein [Tokura99].

Die Besetzung der $A$-Plätze der Manganate $A \mathrm{MnO}_{3}$ spielt also mehrere Rollen. Zum einen bestimmt sie die Valenz des zentralen Manganions, zum anderen bestimmt sie die Struktur und damit auch die Abweichung des Bindungswinkels von $180^{\circ}$ zwischen den Mn-O-Mn-Ionen. Dies wiederum ist für die elektronischen und magnetischen Eigenschaften entscheidend, wie man im Folgenden sehen wird.

\subsubsection{Aufhebung Der Energieentartungen}

Ein neutrales Manganatom hat zusätzlich zur Edelgaselektronenkonfiguration von Argon sieben weitere Elektronen, die gemäß Aufbauprinzip zunächst die beiden 4sOrbitale und anschließend vier der fünf entarteten 3d-Orbitale besetzen, so dass sich eine Elektronenkonfiguration von $[\mathrm{Ar}] 4 \mathrm{~s}^{2} 3 \mathrm{~d}^{5}$ ergibt. Da bei den Manganaten die Valenz durch die $A$-Platzbesetzung bestimmt wird und so die Manganionen in den Oxidationsstufen +3 bzw. +4 vorliegen, ergeben sich dementsprechend Elektronenkonfigurationen von $\mathrm{Mn}^{3+}:[\mathrm{Ar}] 3 \mathrm{~d}^{4}$ bzw. $\mathrm{Mn}^{4+}:[\mathrm{Ar}] 3 \mathrm{~d}^{3}$.

Dadurch dass die Manganionen von Sauerstoffoktaedern umgeben sind, spalten die fünf 3d-Orbitale auf. Die Aufhebung der Entartung durch das Kristallfeld der Sauerstoffionen kann man mit dem Aussehen und der räumlichen Orientierung der 3d-Orbitale anschaulich verstehen: Die Orbitale $\mathrm{d}_{3 z^{2}-r^{2}}$ und $\mathrm{d}_{x^{2}-y^{2}}$ zeigen in Richtung von Sauerstoffionen, so dass diese Orbitale eine stärkere Coulombabstoßung erfahren. Dadurch sind die übrigen drei 3d-Orbitale $\left(\mathrm{d}_{x y}, \mathrm{~d}_{x z}\right.$ und $\left.\mathrm{d}_{y z}\right)$ energetisch günstiger. Das Kristallfeld spaltet die fünf entarteten 3d-Orbitale also in drei energetisch günstigere $t_{2 \mathrm{~g}}$-Niveaus und zwei erhöhte $\mathrm{e}_{\mathrm{g}}$-Niveaus auf, deren Energiedifferenz etwa $\Delta_{\mathrm{CF}} \approx 1 \mathrm{eV}$ ist. Das $\mathrm{Mn}^{4+}$-Ion füllt gemäß Hund'schen Regeln die $\mathrm{t}_{2 \mathrm{~g}}$ mit parallelen Spins, was in einem Rumpfspin von $S=3 / 2$ resultiert. Da die Hund'sche Kopplungsenergie etwa $J_{\mathrm{H}} \approx 2 \mathrm{eV}$ bis $3 \mathrm{eV}$ [Tokura99] ist, besetzt das vierte Elektron eines $\mathrm{Mn}^{3+}$-Ions einen $\mathrm{e}_{\mathrm{g}}$ Zustand, so dass ein High-Spin-Zustand mit $S=2$ entsteht.

Da das $\mathrm{d}_{3 z^{2}-r^{2}}$-Orbital in Richtung von zwei, das $\mathrm{d}_{x^{2}-y^{2}}$ aber in Richtung von vier Sauerstoffionen zeigt, führt das vierte Elektron des $\mathrm{Mn}^{3+}$-Ions allerdings zu einer weiteren Energieaufspaltung. Nach Jahn und Teller [Jahn37] wird die Gesamtenergie des Systems weiter verringert, indem die Energie des besetzten $\mathrm{d}_{3 z^{2}-r^{2}}$ Orbitals gesenkt wird. Dies geschieht über eine Gitterelongation in $z$-Richtung, 
a)

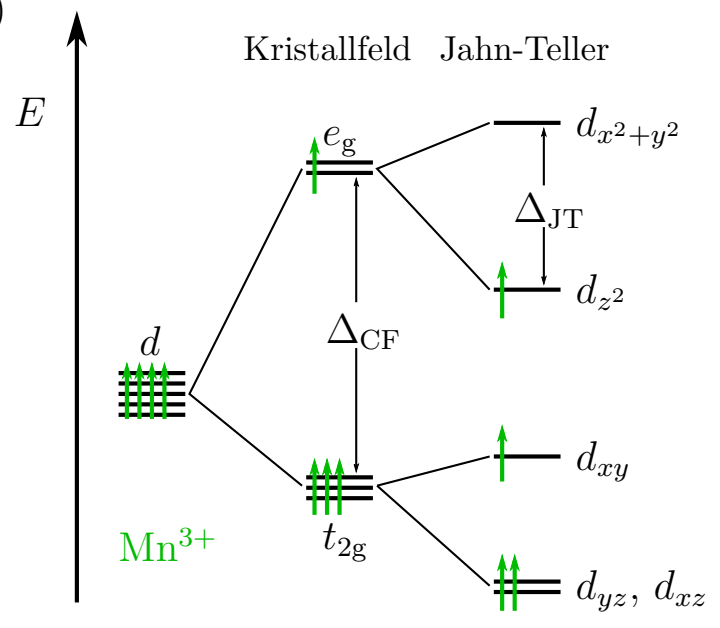

b)

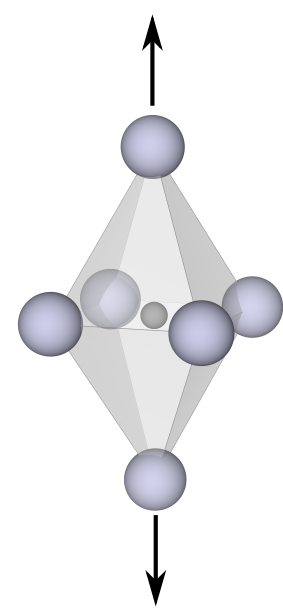

Abbildung 4.2 - a) Aufhebung der Energieentartungen durch Kristallfeld und JahnTeller-Verzerrung am Beispiel eines $\mathrm{Mn}^{3+}$-Ion mit vier Elektronen in 3d-Orbitalen. Die Kristallfeldenergie $\Delta_{C F}$ spaltet die 3d-Niveaus in $t_{2 g^{-}}$und $e_{g^{-N}}$ Niveaus auf. Diese Niveaus spalten durch eine Jahn-Teller-Verzerrung weiter auf, sofern ein $e_{g}$-Niveau besetzt werden muss. b) Die Jahn-Teller-Verzerrung führt zum weiteren Energiegewinn

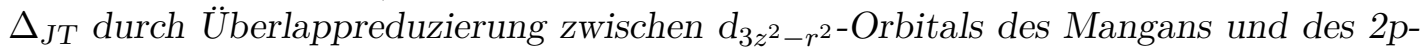
Orbital des Sauerstoffs über Elongation in z-Richtung. Nach [Gross04].

die die Coulombkraft in diese Richtung verringert. Die Elongation in z-Richtung hat allerdings auch zur Folge, dass der Oktaeder in $x$ - und $y$-Richtung gestaucht wird. Dies wiederum verkürzt die Distanz der vier Sauerstoffionen in der Ebene zum $\mathrm{d}_{x y}$-Orbital und vor allem zum unbesetzten $\mathrm{d}_{x^{2}-y^{2}}$, wodurch deren Energien erhöht werden. Abbildung 4.2 zeigt das Resultat. Der Jahn-Teller-Effekt führt $\mathrm{zu}$ einer Jahn-Teller-Aufspaltung von etwa $\Delta_{J T} \approx 0,7 \mathrm{eV}$ [Kovaleva10]. Ohne das vierte Elektron des $\mathrm{Mn}^{3+}$-Ions sind nur die $\mathrm{t}_{2 \mathrm{~g}}$-Orbitale besetzt und es gibt keine Jahn-Teller-Aufspaltung.

\subsubsection{Austauschmechanismen}

Zwei Elektronen an benachbarten Gitterplätzen haben jeweils eine kinetische Energie. Diese Energie kann nach der Heisenberg'schen Unschärferelation erniedrigt werden, indem die Ortsunschärfe erhöht wird. Die Elektronen besetzen dann beide Atome. Das Pauli-Prinzip verbietet dann aber den gleichen Quantenzustand. Im einfachsten Fall haben die Elektronen im Spin-Singlett-Zustand dadurch entgegengesetzte Spins. In diesem Fall kommt hinzu, dass die Aufenthaltswahrscheinlichkeit 
der Elektronen zwischen den Atomen erhöht wird, was zur zusätzlichen Energieerniedrigung führt, da dadurch die Coulomb-Abstoßung kleiner wird. Dadurch, dass sich zwei Orbitale benachbarter Atome überlappen, können sich also magnetische Zustände - in diesem Fall eine antiferromagnetische Kopplung - einstellen.

Der Überlapp der 3d-Orbitale der Mn-Ionen ist in der Perowskitstruktur klein. Quantenmechanische Austauschwechselwirkungen finden hier daher indirekt über die Sauerstoffionen statt, die in der Edelgaskonfiguration $\mathrm{O}^{2-}$ : [He] $2 \mathrm{~s}^{2} 2 \mathrm{p}^{6}$ vorliegen, so dass sie keinen magnetischen Beitrag liefern. Typischerweise konkurrieren bei Austauschmechanismen zwei Energien. Zum einen bringt eine Delokalisierung eines Elektrons über mehrere Atome - wie beschrieben - einen Energievorteil. Zum anderen muss aber aufgrund des Pauli-Prinzips eine hohe Coulomb-Energie bezahlt werden.

Im Folgenden werden zwei Austauschwechselwirkungen vorgestellt, die in den untersuchten Manganaten vorkommen und jeweils zu einer bestimmten magnetischen Ordnung führen.

\section{Superaustausch}

Geht man zunächst von einem reinen $\mathrm{Mn}^{3+}{ }_{-}$Perowskit wie zum Beispiel $\mathrm{LaMnO}_{3}$ aus, findet der Austausch der Elektronen zwischen den 3d-Ionen indirekt über die hybridisierten Orbitale von Mangan und Sauerstoff statt. Reale Hüpfprozesse über ein vollbesetztes Orbital sind energetisch verboten. Aufgrund der Energie-ZeitUnschärfe finden sie trotzdem statt und man spricht vom virtuellen Hüpfen. Das effektive Transferintegral $t_{d d}$ zwischen zwei 3d-Orbitalen folgt aus Störungsrechnungen und ist gegeben durch [Gross04, Bhattachar14]

$$
t_{d d}=\frac{t_{p d}^{2}}{\Delta_{\mathrm{CT}}} .
$$

Ein Transferintegral hängt immer vom Überlapp der Orbitale ab und ist ein Maß für die kinetische Energie, die ein Elektron durch Delokalisierung gewinnt. Daher ist klar, dass das effektive Transferintegral $t_{d d}$ von dem Überlapp $t_{p d}$ zwischen $3 \mathrm{~d}$ Manganion und 2p-Sauerstoffion abhängen muss. Die Energiedifferenz $\Delta_{\mathrm{CT}}$ zwischen 3d- und 2p-Niveaus wird Ladungstransferenergie (engl. charge transfer gap) genannt (s. auch Abb. 4.3) und fließt auch in das effektive Transferintegral ein, da es die Energie ist, die ein Elektron aufwenden muss, um vom Sauerstoff- auf das Manganatom zu springen. [Bhattachar14] 
a)
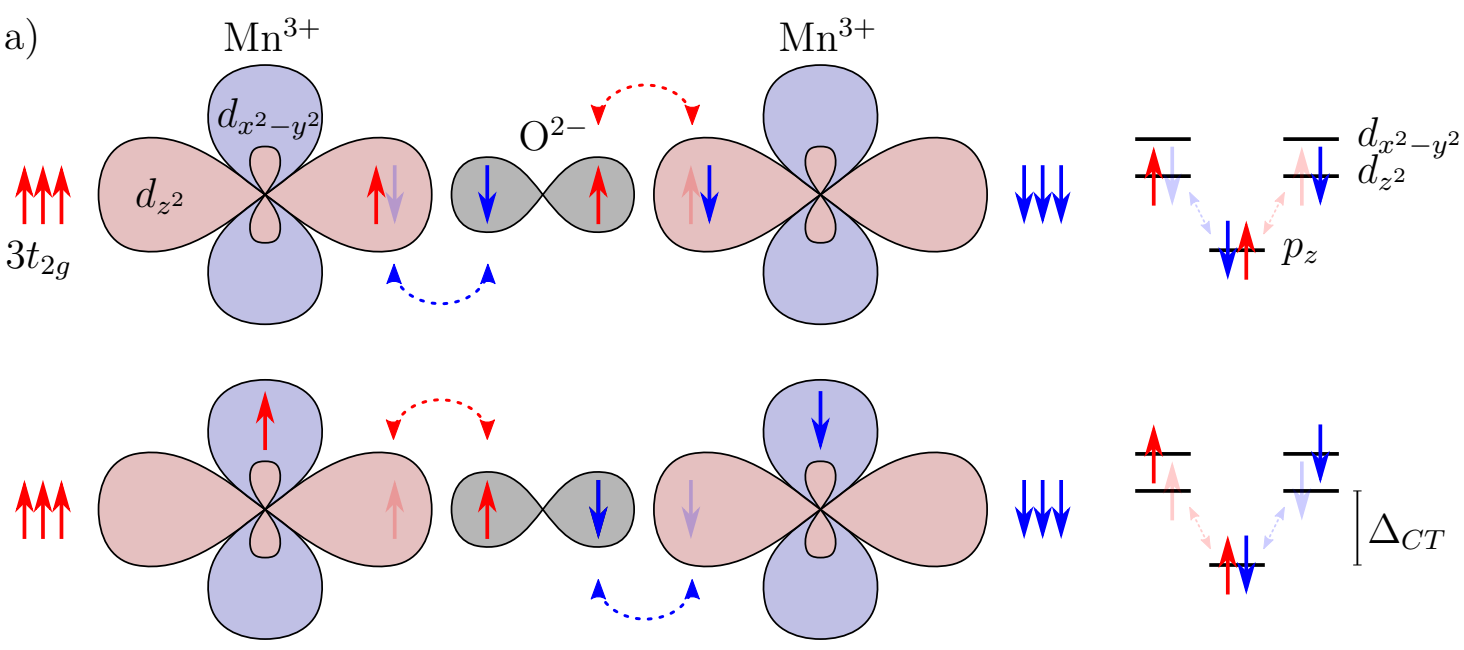

b)
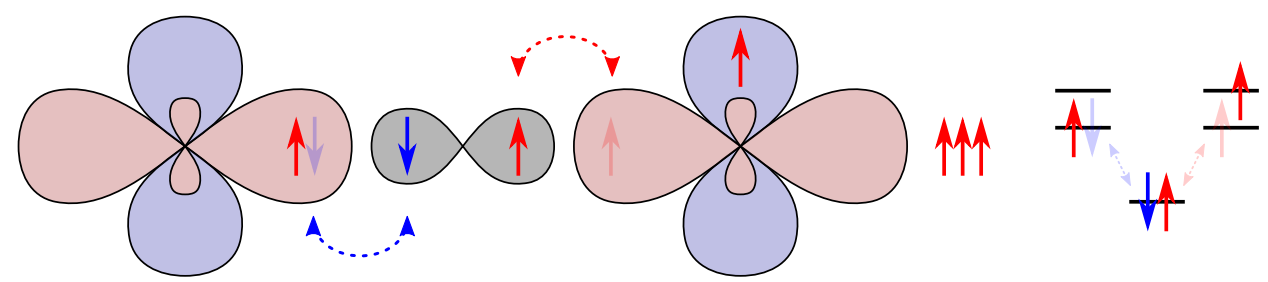

Abbildung 4.3 - Die ersten beiden Goodenough-Kanamori-Anderson-(GKA)-Regeln.

a) Der ersten GKA-Regel folgend koppeln halbvolle $d_{3 z^{2}-r^{2}}$-Orbitale $\left(d_{z^{2}}\right.$ ) (oben) stark antiferromagnetisch über das Sauerstoffion, da eine maximale Energiereduzierung durch Elektrondelokalisierung nur möglich ist, wenn in beiden $\mathrm{Mn}^{3+}$-Ionen gemäß Hund'scher Regel Platz für die jeweilige Spinrichtung der Elektronen ist. Leere $d_{3 z^{2}-r^{2}}$-Orbitale (unten) koppeln ebenso stark antiferromagnetisch, der günstigste $\mathrm{Zu}$ stand ist hier der, bei dem der Rumpfspin dem des hüpfenden Sauerstoffs entspricht. b) Ist ein Orbital leer, das andere halbvoll, so koppeln die Manganionen nach der zweiten GKA-Regel schwach ferromagnetisch. Nach [Gross04].

Die Energie, die aufgrund von Coulomb-Abstoßung bezahlt werden muss, wird üblicherweise als Mott-Hubbard $U$ bezeichnet. Man kann sich nun zwei Extremfälle vorstellen:

Ist $\Delta_{\mathrm{CT}} \gg U$ spricht man von einem Mott-Hubbard-Isolator. Ein großes $\Delta_{\mathrm{CT}}$ führt dazu, dass das Transferintegral $t_{d d}$ klein wird, so dass die Coulombenergie nicht mehr überwunden werden kann $U \gg t_{d d}$. Die Elektronen lokalisieren und das System ist isolierend, wobei die Bandlücke zwischen Valenz- und Leitungsband in der Größenordnung von $U$ liegt. Die Energiereduzierung durch virtuelles Hüpfen kann mit einer Störungsrechnung zweiter Ordnung bestimmt werden [Gross04, Bhattachar14]: $J_{\mathrm{SE}}=-2 t_{d d}^{2} / U$.

Auch für $\Delta_{\mathrm{CT}} \ll U$ liegt isolierendes Verhalten vor, sofern auch $\Delta_{\mathrm{CT}} \gg t_{d d}$. In 
diesem Fall liegt ein Ladungstransfer-Isolator vor. Hier ist die Energielücke in der Größenordnung von $\Delta_{\mathrm{CT}}$.

Der Unterschied beider Isolatoren liegt nur darin, ob das Loch, das durch ein virtuelles Hüpfen entsteht, auf dem Sauerstoff (Ladungstransfer-Isolator) oder dem Mangan (Mott-Hubbard-Isolator) liegt. Beide Isolatoren favorisieren einen antiferromagnetischen Zustand, so dass magnetisch kein Unterschied zu sehen ist.

Um die magnetischen Wechselwirkungen in den Manganaten abzuschätzen, können zwei der drei Goodenough-Kanamori-Anderson-(GKA)-Regeln [Anderson50, Goodenough55, Kanamori59] herangezogen werden. Die dritte GKA-Regel behandelt den Fall eines Bindungswinkels von $90^{\circ}$, der in den vorliegenden Perowskiten nicht vorkommt.

Abbildung 4.3 zeigt schematisch die ersten zwei GKA-Regeln, repräsentiert durch drei verschiedene Konfigurationen. Diese zeigen jeweils zwei $\mathrm{Mn}^{3+}$-Ionen, zwischen denen ein $\mathrm{O}^{2-}$-Ion liegt. Der Rumpfspin wird dabei jeweils durch drei $t_{2 \mathrm{~g}}$-Spins dargestellt. Aufgrund der großen Hund'schen Kopplung muss die gleiche Spinorientierung in den $\mathrm{e}_{\mathrm{g}}$-Orbitalen $\mathrm{d}_{x^{2}-y^{2}}$ und $\mathrm{d}_{3 z^{2}-r^{2}}$ herrschen. Virtuelle Hüpfprozesse sind durch gestrichelte Pfeile dargestellt. Auf der rechten Seite der Abbildung sind die zugehörigen Energiebesetzungen skizziert.

Erste GKA-Regel [s. Abb. 4.3 a)]:

Der $180^{\circ}$-Austausch zwischen gefüllten (leeren) $\mathrm{d}_{3 z^{2}-r^{2}}$-Orbitalen ist stark und antiferromagnetisch.

Befindet sich in beiden $\mathrm{d}_{3 z^{2}-r^{2}}$-Orbitalen jeweils ein (kein) Elektron, so ist der energetisch günstigste Zustand der, in dem beide Elektronen des $\mathrm{p}_{z}$-Orbitals des Sauerstoffions in das jeweilig gegenüberliegende $\mathrm{d}_{3 z^{2}-r^{2}}$-Orbital delokalisieren. Da das $\mathrm{p}_{z}$-Orbital nur zwei Elektronen aufnehmen kann, sind deren Spins entgegengesetzt. Das Hüpfen in die Manganionen ist daher am günstigsten, wenn auch deren Rumpfspins antiferromagnetisch zueinander stehen. Sind beide $\mathrm{d}_{3 z^{2}-r^{2}}$-Orbitale unbesetzt, entspricht die Spinorientierung der hüpfenden Sauerstoffelektronen der des jeweiligen Rumpfspins. Sind beide $\mathrm{d}_{3 z^{2}-r^{2}}$-Orbitale besetzt, sind die hüpfenden Spins aufgrund des Pauli-Prinzips antiferromagnetisch zum Rumpfspin ausgerichtet.

Zweite GKA-Regel [s. Abb. 4.3 b)]:

Der $180^{\circ}$-Austausch zwischen einem gefüllten und einem leeren $\mathrm{d}_{3 z^{2}-r^{2}}$ Orbital ist schwach und ferromagnetisch.

Die linke Seite der Situation aus Abbildung 4.3 b) entspricht der ersten Situation aus a). Der Sauerstoffspin ist antiferromagnetisch zum Rumpfspin des Mangans. 
Da das $\mathrm{d}_{3 z^{2}-r^{2}}$-Orbital des rechten Mangans frei ist, kann das Elektron unabhängig vom Rumpfspin in das $\mathrm{d}_{3 z^{2}-r^{2}}$-Orbital hüpfen. Allerdings kann die Hund'sche Kopplungsenergie $J_{\mathrm{H}}$ gewonnen werden, wenn der Rumpfspin dem Spin des Elektrons entspricht. Dadurch gibt sich eine ferromagnetische Kopplung. Der effektive Energiegewinn ist in diesem Fall $J_{\mathrm{SE}} \approx 2 t_{\mathrm{dd}}^{2} J_{\mathrm{H}} / U^{2}$. Dadurch, dass $J_{\mathrm{H}} / U \ll 1$, ist diese Kopplung allerdings schwach.

\section{Doppelaustausch}

Im Gegensatz zum Superaustausch beschreibt der Doppelaustausch reale Hüpfprozesse. Dazu ist notwendig, dass die $\mathrm{e}_{\mathrm{g}}$-Orbitale eines Manganions unbesetzt bleiben. Dies kann über Dotierung der A-Plätze mit $\mathrm{Sr}^{2+}$ erreicht werden. Abbildung 4.4 beschreibt die Situation. Vor dem Hüpfen befindet sich auf der linken Seite ein $\mathrm{Mn}^{3+}$ Ion mit einem Elektron im $\mathrm{d}_{3 z^{2}-r^{2}}$-Orbital, auf der rechten Seite ein $\mathrm{Mn}^{4+}$-Ion ohne $\mathrm{e}_{\mathrm{g}}$-Besetzung und dazwischen wieder ein vollbesetztes Sauerstoffion. Dadurch, dass hier ein Elektron fehlt, kann das Elektron - wie dargestellt - über das Sauerstoffion ins $\mathrm{Mn}^{4+}$-Ion hüpfen. Dadurch ergibt sich die Situation auf der rechten Seite der Abbildung. Der Anfangszustand entspricht dem Endzustand, sie sind energetisch entartet und können daher - im Gegensatz zum Superaustausch - real stattfinden. Auch hier wird die Energie durch Delokalisierung reduziert. Diese Situation erinnert an Abbildung $4.3 \mathrm{~b}$ ), denn auch hier ist aufgrund der Hund'schen Kopplungsenergie eine ferromagnetische Situation bevorzugt. Da es sich hier um einen realen Prozess handelt, ist eine antiferromagnetische Ausrichtung der Mn-Ionen nicht möglich, da in diesem Fall kein Spin- $\downarrow$ aus dem $\mathrm{Mn}^{3+}$ in das $\mathrm{Mn}^{4+}$ hüpfen kann, da nur ein Spin- $\uparrow$ vorhanden ist.

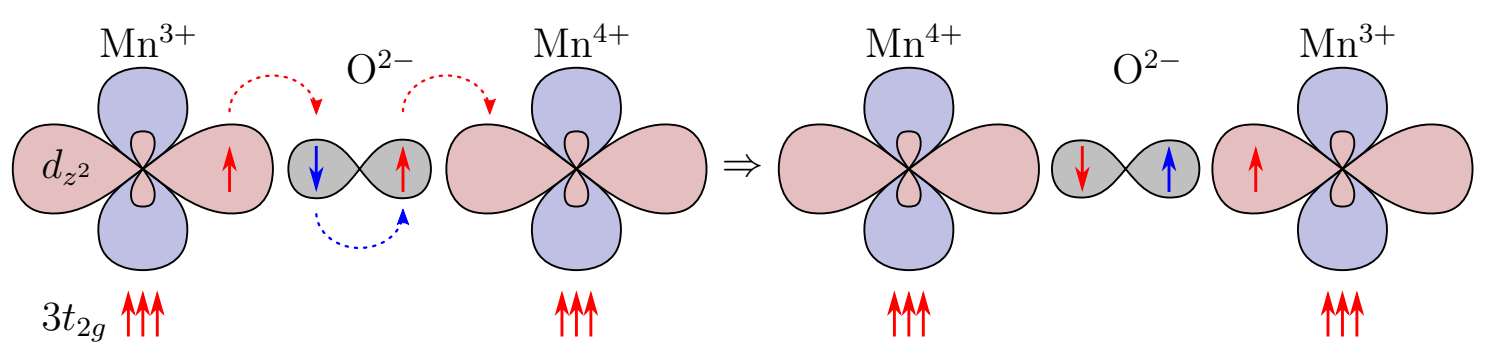

Abbildung 4.4 - Beim Doppelaustausch sind reale Sprünge vom $d_{3 z^{2}-r^{2}}$-Orbital $\left(d_{z^{2}}\right)$ des $\mathrm{Mn}^{3+}$-Ions in das des $\mathrm{Mn}^{4+}$-Ions - sofern die Rumpfspins parallel zueinander sind - möglich, da die Zustände vorher und nachher entartet sind. Nach [Gross04].

Man kann erkennen, dass sich die ferromagnetische Spinausrichtung und die Beweglichkeit der Elektronen und damit eine hohe elektrische Leitfähigkeit bedingen. 
Anderson und Hasegawa [Anderson55] betrachten die tatsächliche Ausrichtung der Rumpfspins zueinander und geben ein effektives Transferintegral

$$
t_{\mathrm{dd}}^{\mathrm{eff}}=t_{\mathrm{dd}} \frac{\theta}{2}
$$

an, das vom Winkel $\theta$ benachbarter $t_{2 \mathrm{~g}}$-Spins abhängt. Thermisch bedingte Abweichungen von der Idealbedingung $\theta=0$ erhöhen also den elektrischen Widerstand. Andersherum können externe Magnetfelder Rumpfspins gleichrichten und damit den Widerstand reduzieren. Da gerade in der Nähe der Curie-Temperatur $T_{\mathrm{C}}$ die Abweichung vom idealen Winkel groß ist, sollte der Effekt des externen Magnetfeldes hier am größten sein, was experimentell nachgewiesen ist [Moshnyaga07]. Damit ist das Doppelaustauschmodell das erste Modell, das den sogenannten kolossalen Magnetwiderstand ansatzweise erklären kann.

Allerdings weicht der theoretisch vorhergesagte CMR-Wert deutlich von experimentellen Werten ab. Daher müssen weitere Einflüsse betrachtet werden. Millis et al. betrachten zum Beispiel den Widerstand eines $\mathrm{La}_{1-x} \mathrm{Sr}_{x} \mathrm{MnO}_{3}$-Systems im Bereich $0,2 \leq x \leq 0,4$ und schlagen vor, zusätzlich zum Doppelaustauschmodell auch starke Elektron-Phonon-Kopplung aufgrund der Jahn-Teller-Verzerrung als wesentlichen Bestandteil der Widerstandsänderung anzusehen [Millis95]. Später führt Millis einen dimensionslosen Parameter $\lambda=E_{\text {latt }} / t_{\mathrm{dd}}^{\text {eff }}$ [Millis98] ein, der die Energie $E_{\text {latt }}$ enthält, die man durch Lokalisieren eines Elektrons in einer Jahn-Teller-Verzerrung gewinnt. Da das Transferintegral $t_{\mathrm{dd}}^{\mathrm{eff}}$ über $t_{\mathrm{dd}}$ auch vom Mn-O-Mn-Bindungswinkel abhängt, kann über Dotierung der A-Platz-Ionen die Elektron-Phonon-Kopplung verändert werden. Moshnyaga und Samwer [Moshnyaga11] haben dazu ein generisches Phasendiagramm mit Daten einiger Manganate von [Hwang95] auf Basis von [Mathur03] erstellt (s. Kap. 4.1.5).

\subsubsection{POLARONEN}

Bei der beschriebenen Jahn-Teller-Verzerrung ändert ein einzelnes Elektron seine eigene Umgebung und dadurch auch den Überlapp seines Orbitals mit denen der Nachbaratome. Dadurch lokalisiert es sich selbst in einer Gitterverzerrung. Es entsteht ein Quasiteilchen namens Jahn-Teller-Polaron. Durch die lokale Gitterveränderung hat ein Jahn-Teller-Polaron auch Einfluss auf umliegende Gitteratome oder sogar andere Jahn-Teller-Polaronen. Dadurch können sogenannte korrelierte Jahn-Teller-Polaronen (s. Abb. 4.5) entstehen, die man häufig im Zusammenhang mit dem CMR sieht und verstärkt im Bereich um $T_{\mathrm{C}}$ misst. Korrelierte Polaronen 
werden allerdings nur in Systemen mit großer Elektron-Phonon-Kopplung beobachtet. Nelson et al. haben Korrelationslängen korrelierter Jahn-Teller-Polaronen in $\mathrm{La}_{0,7} \mathrm{Ca}_{0,3} \mathrm{MnO}_{3}$ oder $\mathrm{Pr}_{0,7} \mathrm{Ca}_{0,3} \mathrm{MnO}_{3}$ von ein bis zwei Gitterkonstanten gemessen. Korrelierte Polaronen zeichnen sich durch eine große Elektron-PhononWechselwirkung aus und sind daher in LSMO nicht zu erwarten. In LSMO sind allerdings - vor allem auch über $T_{\mathrm{C}}$ - große Polaronen (engl. large Polarons) nachgewiesen worden [Hartinger04, Mannella04, Mannella05, Hartinger06].

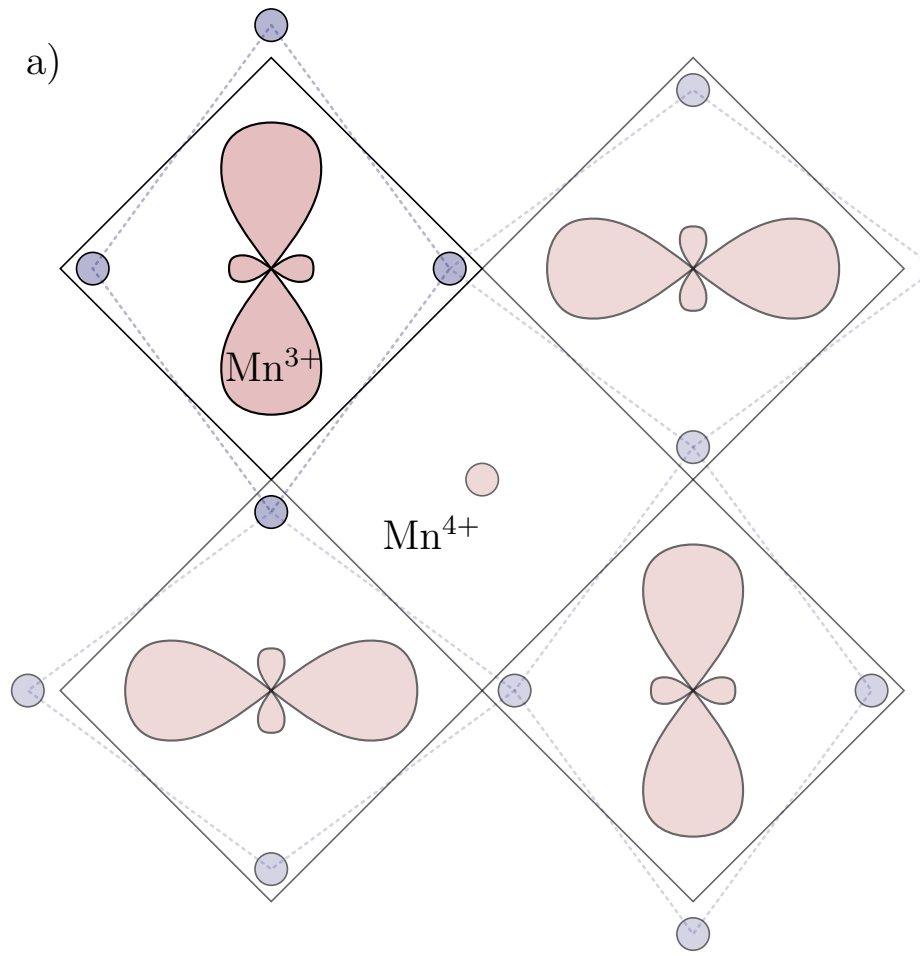

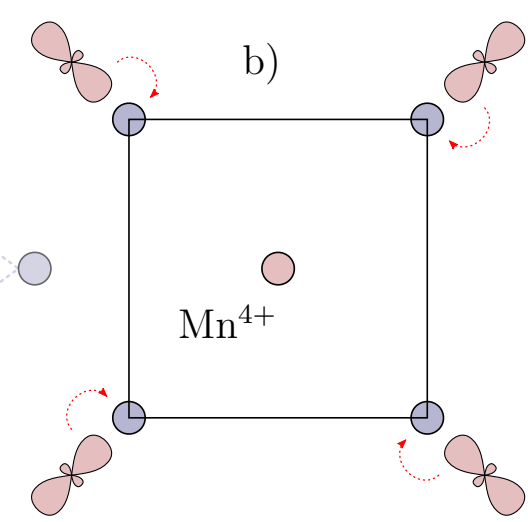

c)

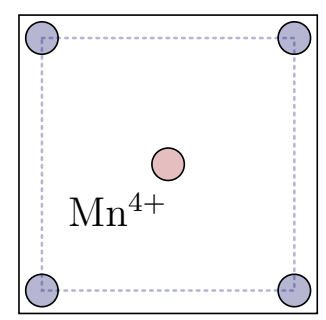

Abbildung 4.5 - Bei Polaronen führt ein Elektron bzw. ein Loch zu einer Variation der Eigenschaften umgebender Atome. a) Ein Jahn-Teller-Polaron kann über Gitterverzerrungen mit anderen zu einem korrelierten Zustand führen. b) Bei einem orbi-

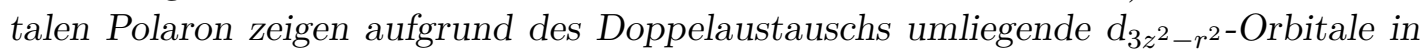
Richtung des $\mathrm{Mn}^{4+}$-Ions. c) Der kleinere Ionenradius des $\mathrm{Mn}^{4+}$-Ion führt zu einem dielektrischen Polaron. Nach [Gross04].

Auch andere Polaronen sind denkbar. Abbildung 4.5 zeigt neben (korrelierten) Jahn-Teller-Polaronen auch ein orbitales Polaron [b)], das aufgrund des Doppelaustausches entstehen kann. Dabei zeigen die besetzten $\mathrm{d}_{3 z^{2}-r^{2}}$-Orbitale der umliegenden $\mathrm{Mn}^{3+}$-Ionen in Richtung des zentralen $\mathrm{Mn}^{4+}$-Ions. Abbildung 4.5 c) zeigt ein dielektrisches Polaron, das aufgrund des kleineren Ionenradius des $\mathrm{Mn}^{4+}$-Ions umgebende Sauerstoffionen näher heranrücken lässt. 


\subsubsection{PHASENDIAGRAMM}

An den dargestellten Überlegungen können nun Bereiche des Phasendiagramms von $\mathrm{La}_{1-x} \mathrm{Sr}_{x} \mathrm{MnO}_{3}$ verstanden werden. Abbildung 4.6 zeigt dieses in Abhängigkeit von der Dotierung. Ein reines Lanthanmanganoxid (LMO, $x=0$ ) zeigt unterhalb von $T_{\mathrm{OO}}=780 \mathrm{~K}$ [Murakami98] orbitale Ordnung und dadurch eine JahnTeller-verzerrte orthorhombische Struktur. Aufgrund der kooperativen Jahn-TellerVerzerrung zeigen in der Ebene leere $\mathrm{d}_{x^{2}-y^{2}}$ Orbitale auf einfach besetzte $\mathrm{d}_{3 z^{2}-r^{2}}$ Orbitale, so dass sich nach der zweiten GKA-Regel eine ferromagnetische Kopplung einstellt. Unterhalb der Néel-Temperatur $T_{\mathrm{N}}=140 \mathrm{~K}$ ordnen die Manganionen in c-Richtung gemäß zweiter GKA-Regel antiferromagnetisch, da zwischen den Ebenen immer leere Orbitale aufeinander zeigen. Dadurch ergibt sich ein A-TypAntiferromagnet. Aufgrund der Coulomb-Wechselwirkung lokalisieren die Elektronen und es ergibt sich ein Mott-Hubbard-Isolator.

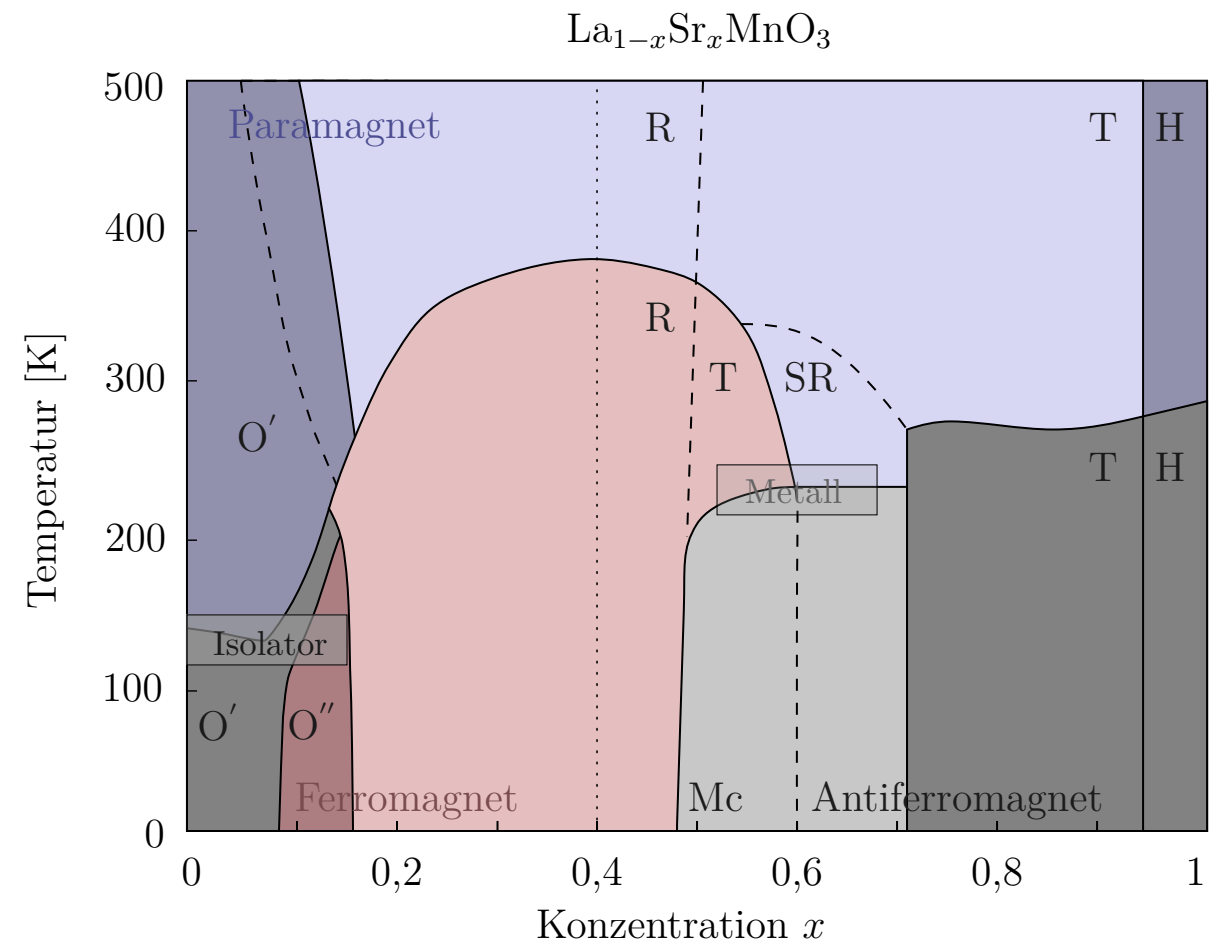

Abbildung 4.6 - Das $\mathrm{La}_{1-x} \mathrm{Sr}_{x} \mathrm{MnO}_{3}$-Phasendiagramm enthält ferromagnetische (rot), paramagnetische (blau) und antiferromagnetische (grau) Bereiche, die entweder metallisch (hell) oder isolierend (dunkel) sind. Unterschiedliche Symmetrien stellen sich ein: Jahn-Teller verzerrt orthorhombisch $\left(O^{\prime}\right)$, orbital-geordnet orthorhombisch $\left(O^{\prime \prime}\right)$, rhomboedrisch $(R)$, tetragonal $(T)$, monoklin $(M c)$ und hexagonal $(H)$. Nach [Hemberger02]. 
Auf der anderen Seite des Phasendiagramms steht ein reines Strontiummanganoxid (SMO, $x=1$ ). Hier stehen nur $\mathrm{Mn}^{4+}$-Ionen auf den B-Plätzen, so dass alle $\mathrm{e}_{\mathrm{g}}$-Orbitale unbesetzt sind. Dadurch stellt sich nach der ersten GKA-Regel eine antiferromagnetische Kopplung zu allen nächsten Mangannachbarn ein. Dadurch bildet sich ein G-Typ-Antiferromagnet mit einer Néel-Temperatur von $T_{\mathrm{N}}=260 \mathrm{~K}$ [Takeda74]. Dadurch, dass keine $\mathrm{e}_{\mathrm{g}}$-Elektronen vorhanden sind, bildet sich ein Bandisolator. Dadurch, dass hier der Goldschmidt'sche Toleranzfaktor bei $t \approx 1,02$ liegt ${ }^{3}$, ist die Struktur hexagonal. Es gibt allerdings auch kubische Modifikationen [Syono69].

Die ferromagnetischen Bereiche zwischen $x=0,17$ und $x=0,6$ können mit dem Doppelaustauschmodell verstanden werden. Hier geht die magnetische Phase mit beweglichen Elektronen einher, so dass metallisches Verhalten beobachtet wird. Die maximale Curie-Temperatur $T_{\mathrm{C}} \approx 370 \mathrm{~K}$ kann im Dotierungsbereich von $x=0,3$ bis $x=0,4$ erreicht werden.

In allen weiteren Bereichen wird zum einen das Konkurrenzverhalten zwischen Doppelaustausch und Superaustausch zu komplex, zum anderen müssen auch Clusterbildung [Burgy01], Phasenseparationen [Yunoki98] und strukturelle Veränderungen aufgrund des Toleranzfaktors - der von 0,89 auf 1,02 steigt - mit einhergehenden Mn-O-Mn-Winkeländerungen berücksichtigt werden.

In der vorliegenden Arbeit wird eine LSMO-Probe verwendet, die eine Dotierung von $x=0,4$ aufweist. Die vertikale gepunktete Linie in Abbildung 4.6 zeigt, dass diese Probe einen Phasenübergang von einer ferromagnetischen Phase zu einer paramagnetischen Phase zeigt, dabei aber stets metallisches Verhalten aufweist.

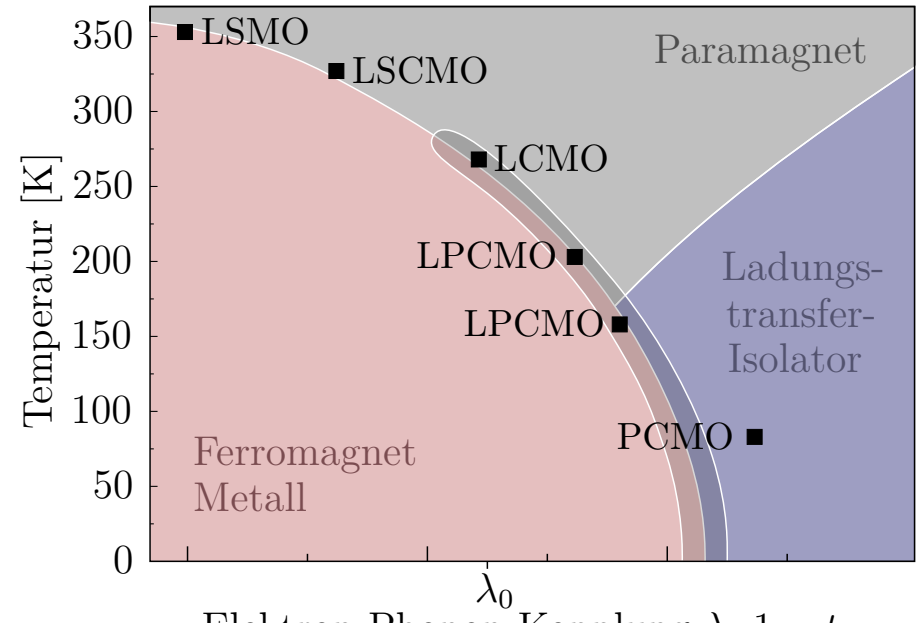

Elektron-Phonon-Kopplung $\lambda, 1-t$
Abbildung 4.7 - Ein generisches Phasendiagramm der Manganate, in dem die Elektron-Phonon-Kopplung gegen die Temperatur aufgetragen ist. Für hohe Temperaturen sind die Systeme paramagnetisch, für tiefe entweder ferromagnetisch metallisch oder ladungsgeordnet isolierend. Nach [Moshnyaga11]

\footnotetext{
${ }^{3}$ Werte der Radien aus [Shannon76].
} 
Abbildung 4.7 zeigt ein generisches Littlewood-Mathur-Phasendiagramm [Mathur03] mit experimentellen Daten von Proben perfekter Dotierung [Moshnyaga11]. Aufgetragen ist die Elektron-Phonon-Kopplung $\lambda$ gegen die Temperatur T. Mit CurieTemperaturen einiger Manganate (Daten von [Hwang95]) entstehen drei Bereiche. Bei hohen Temperaturen über $T_{C}$ stellt sich grundsätzlich eine paramagnetische Phase ein. Bei hohen Elektron-Phonon-Kopplungen lokalisieren die Elektronen und es stellt sich ein ladungsgeordneter isolierender Zustand ein. Für kleinere $\lambda$ ist der Grundzustand metallisch und ferromagnetisch. Unterhalb $\lambda_{0}$ sind die Phasenübergänge zweiter Ordnung und somit kontinuierlich. Der dunkel hinterlegte Übergangsbereich zeichnet sich durch Übergänge erster Ordnung aus, was mit Hysteresen einhergeht. Für eine Probe der Dotierung $x=0,4$ wird also eine kleine aber konstante Elektron-Phonon-Kopplung nahe des Phasenübergangs, der von zweiter Ordnung ist, erwartet.

\subsection{Metallische Gläser}

Ein weiteres untersuchtes Probensystem ist $\mathrm{Pd}_{77,5} \mathrm{Cu}_{6} \mathrm{Si}_{16,5}$ - zum einen in kristalliner Form, zum anderen im amorphen Zustand. Allgemein bezeichnet man einen Stoff als amorph, wenn dessen Struktur keine wiederkehrende Gestalt - wie ein Kristall - annimmt, sondern ein unregelmäßiges Muster bildet. Diese Strukturen verfügen also nur über Nahordnung, aber nicht über Fernordnung wie Kristalle. Gläser sind typische amorphe Materialien. Abbildung 4.8 zeigt Siliciumdioxid in kristalliner und amorpher Form, wobei man letzteres auch Quarzglas nennt.

Abbildung 4.8 - Schematischer Ver-

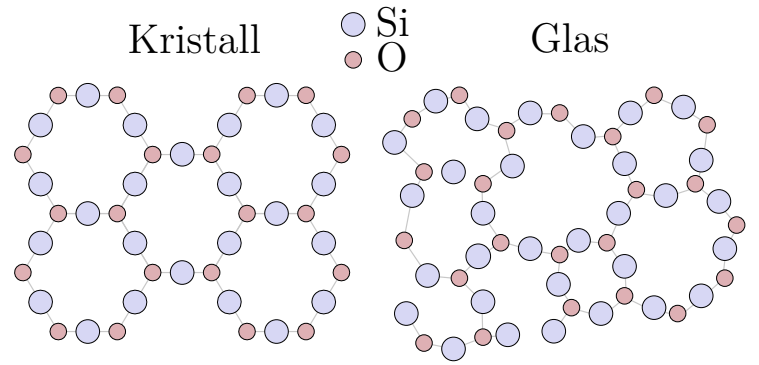

Kühlt man ein System aus der Schmelze ab, so kristallisiert es im Gleichgewicht, sobald die Enthalpie der festen Phase kleiner ist als die der Schmelze. In Abbildung 4.9 geschieht dies bei der Temperatur $T_{\mathrm{m}}$. Die Unstetigkeit des Volumens bzw. der Entropie zeigt, dass es sich dabei um einen Phasenübergang erster Ordnung handelt. Möchte man nun einen amorphen Zustand herstellen, muss die Abkühlrate 


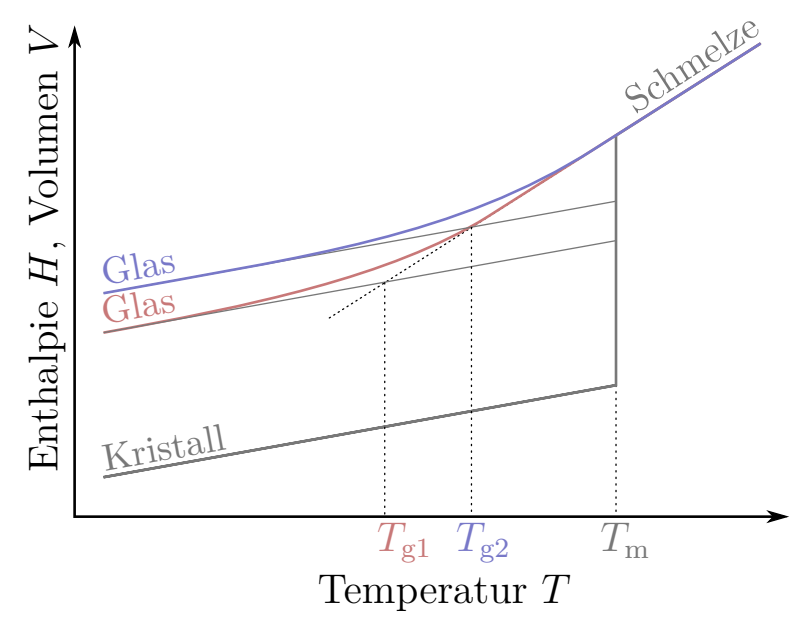

Abbildung 4.9 - Enthalpie bzw. Volumen eines Systems bei konstantem Druck in Abhängigkeit von der Temperatur. Kühlt man ein System aus der Schmelze ab, kristallisiert es. Ist die Abkühlgeschwindigkeit groß genug, entsteht ein Glas. Je größer die Abkühlgeschwindigkeit, desto höher die Glasübergangstemperatur $T_{g}$.

derart erhöhen werden, dass die Viskosität hoch genug ist, um eine Kristallisation zu verhindern. Diese Abkühlraten sind stark vom System abhängig. Sie liegen bei klassischem Fensterglas bei $1 \mathrm{~K} \mathrm{~min}^{-1}$, bei vielen amorphen Metallen hingegen bei über $1000 \mathrm{~K} \mathrm{~s}^{-1}$.

Bei derartigen Kühlraten geht das System unterhalb $T_{\mathrm{m}}$ zunächst in die unterkühlte Schmelze über. Das Volumen bzw. die Enthalpie folgen hier zunächst dem linearen Verlauf der Schmelze. Kühlt man das System weiter ab, wird eine Temperatur $T_{\mathrm{g}}$ erreicht, bei der sich die Steigung ändert. Man erreicht einen metastabilen amorphen Zustand. Die Glasübergangstemperatur $T_{\mathrm{g}}$ ist dabei stark von der Kühlrate abhängig. Je kleiner diese ist, desto mehr Zeit gibt man dem System, in energetisch günstigere Zustände überzugehen. Daher ist die Glasübergangstemperatur größer, je größer die Kühlrate ist. Dadurch wird auch klar, dass der Glasübergang kein thermodynamischer Phasenübergang ist.

Da die Glasübergangstemperatur von experimentellen Zeitskalen abhängt, wird sie häufig auch über die Viskosität

$$
\eta\left(T_{\mathrm{g}}\right)=1 \cdot 10^{12} \mathrm{Pas}
$$

oder die Relaxationszeit

$$
\tau_{\alpha}\left(T_{\mathrm{g}}\right)=100 \mathrm{~s}
$$

eines $\alpha$-Prozesses definiert. Kommt man $T_{g}$ nahe, ändert sich die Viskosität stark über mehrere Größenordnungen. Starke Glasbildner wie Siliciumdioxid folgen dabei 
Abbildung 4.10 - Der AngellPlot ist eine Arrheniusauftragung, bei der zwischen fragilen und starken Glasbildnern unterschieden werden kann. Starke Glasbildner zeigen lineares Verhalten, fragile weichen davon ab. Nach [Angell95].

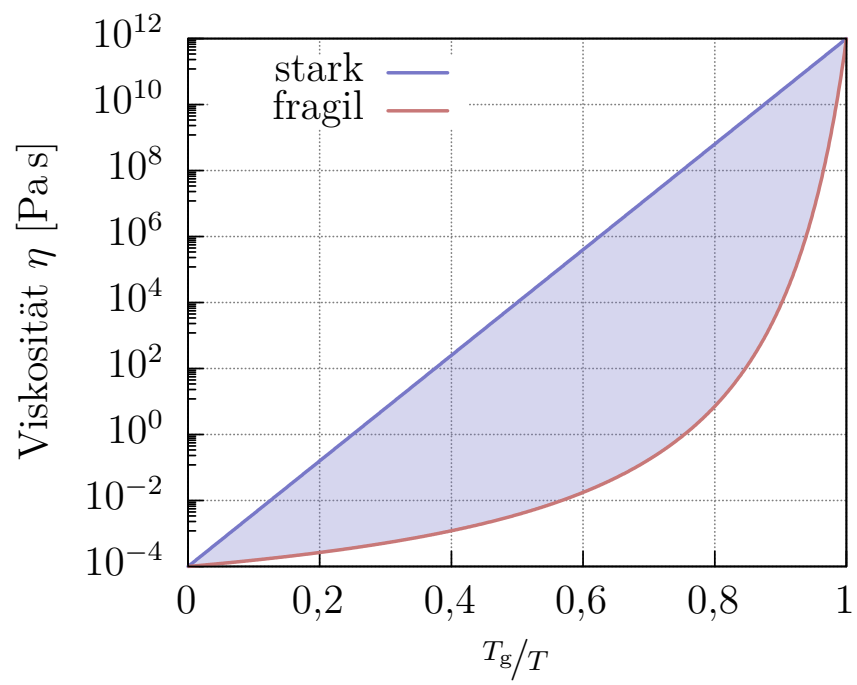

einem Arrhenius-Verhalten [Debenedett01]

$$
\eta=\eta_{0} \exp ^{\frac{E}{k_{\mathrm{B}} T}}
$$

andere Systeme werden besser durch das Vogel-Fulcher-Tammann-Gesetz [Vogel21, Fulcher25, Tammann26, Debenedett01]

$$
\eta=\eta_{0} \exp \frac{D}{T-T_{0}}
$$

mit materialspezifischen Konstanten $\eta_{0}, D$ und $T_{0}$, beschrieben. Mit einem AngellPlot wie in Abbildung 4.10 können starke und fragile Glasbildner qualitativ unterschieden werden. Eine quantitative Beschreibung ist mit der Fragilität

$$
m=\left(\frac{\mathrm{d} \log \eta}{\mathrm{d} \frac{T_{\mathrm{g}}}{T}}\right)_{T=T_{\mathrm{g}}}
$$

möglich, die der Steigung der Kurven im Angell-Plot bei der Glasübergangstemperatur $T_{\mathrm{g}}$ entspricht.

Krausser et al. präsentieren eine analytische Beschreibung, die die Viskosität $\eta$ - mit Gleichung 4.3 somit auch mit der Fragilität - eines metallischen Glases mit der effektiven Ion-Ion-Wechselwirkung verknüpft [Krausser15]. Dabei wird der Schermodul

$$
G(T)=C_{\mathrm{G}} \exp \left[\alpha_{T}(2+\lambda)\left(T_{\mathrm{g}}-T\right)\right]
$$


mit dem thermischen Ausdehnungskoeffizienten $\alpha_{T}$, einem temperaturunabhängigen Vorfaktor $C_{\mathrm{G}} \propto \exp \left[-\alpha_{T} T_{\mathrm{g}}(2+\lambda)\right]$, über eine Aktivierungsenergie $E(T)$ kooperativer Umordnungen mit der Viskosität $\eta$ verknüpft. Für diese gilt schließlich

$$
\frac{\eta(T)}{\eta_{0}}=\exp \left[\frac{V_{\mathrm{c}} C_{\mathrm{G}}}{k T} \exp \left[\alpha_{T}(2+\lambda)\left(T_{\mathrm{g}}-T\right)\right]\right]
$$

wobei hier $\eta_{0}$ eine Normalisierungkonstante und $V_{\mathrm{c}}$ das charakteristische Volumen ist. Der Parameter $\lambda$ ist proportional zur Steigung des ersten Peaks der radialen Verteilungsfunktion $g(r)$ und wird als Fit-Parameter verwendet. Krausser et al. konnten zeigen, dass Messdaten verschiedener metallischer Gläser durch die analytische Beziehung sehr gut beschrieben werden.

\subsubsection{Potentielle Energielandschaft}

Das Konzept der potentiellen Energielandschaft (PEL) beschreibt qualitativ und quantitativ mechanische Eigenschaften und Relaxationsprozesse eines Glases. Es basiert auf der Arbeit von [Goldstein69], in der er eine Potentialstruktur vorschlug, deren Energiebarrieren im Vergleich zur thermischen Energie groß sind, so dass das System bei tiefen Temperaturen lange im metastabilen Zustand bleibt, ohne zu kristallisieren. Das Konzept wird häufig verwendet und weiterentwickelt, um Eigenschaften der Gläser zu erklären [Angell88, Stillinger95, Debenedett01].

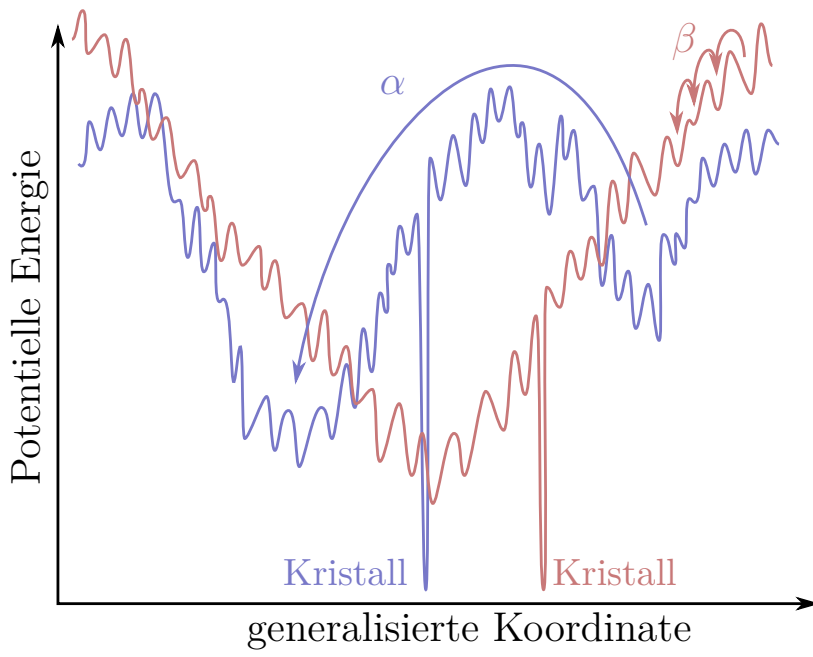

Abbildung 4.11 - Potentielle Energielandschaften von fragilem (blau) und starkem (rot) Glasbildner. Ein Glas kann bei ausreichender Energie in niedrigere Zustände fallen - im Extremfall auch kristallisieren. Nach [Debenedett01].

Die PEL wird über eine Funktion $V\left(\overrightarrow{r_{1}}, \ldots, \overrightarrow{r_{N}}\right)$ beschrieben, die von den Vektoren $\overrightarrow{r_{i}}$ abhängt, die Positions-, Orientierungs- und Vibrationsinformationen jedes der $N$ Teilchen des Systems enthält. Somit ergibt sich eine multidimensionale Ober- 
fläche, die im einfachsten Fall - ohne Orientierungs- und Vibrationsfreiheitsgerade $-3 N+1$-dimensional ist [Debenedett01].

Potentielle Energielandschaften zeichnen sich durch verschiedene Typen lokaler Minima aus. Zum einen gibt es große Täler niedriger Energien, zum anderen eine kleinere Unterstruktur mit deutlich kleineren Energiebarrieren, die der gröberen Struktur aufgeprägt ist. Das tiefste Minimum entspricht der kristallinen Konfiguration. Bei konstantem Volumen ist die PEL eines Systems konstant. Dessen dynamisches Verhalten hängt nun davon ab, wie es sich im PEL in Abhängigkeit von der Temperatur bewegt. Bei hohen Temperaturen kann das System die größten Energiebarrieren in der PEL überwinden, da die thermische Energie größer ist - man spricht von $\alpha$-Relaxationen (vgl. Abb. 4.11). Wird die Temperatur reduziert, können die größten Energiebarrieren nicht mehr überwunden werden. Einige Energiebarrieren sind allerdings immer noch kleiner als $k_{\mathrm{B}} T$, so dass $\beta$-Relaxationsprozesse immer noch stattfinden können. Wird dem System bei hohen Temperaturen genug Zeit gegeben, kann es die tiefsten Energien erreichen und am Ende kristallisieren. Erhöht man aber die Kühlrate stark genug, bleibt das System in einem lokalen Minimum und damit in einem amorphen Zustand gefangen.

Die PEL von starken und fragilen Glasbildnern unterscheiden sich deutlich. Ein starker Glasbildner folgt der Arrhenius-Gleichung. Daher ist die Aktivierungsenergie der Prozesse konstant über die Temperatur. Die zugehörige PEL muss also homogen sein. Ein fragiler Glasbildner hingegen zeichnet sich durch temperaturabhängige Aktivierungsenergien aus und man erwartet eine heterogene PEL. Abbildung 4.11 zeigt schematisch Energielandschaften, die einen starken (rot) und einen fragilen Glasbildner (blau) darstellen [Debenedett01]. 



\section{Probenherstellung Und -CHARAKTERISIERUNG}

Nach den theoretischen Beschreibungen der untersuchten Proben sollen nun Verfahren erläutert werden, die zur Herstellung der jeweiligen Proben verwendet worden sind. Grundsätzlich muss nach der Herstellung einer Probe die Qualität - also Reinheit, Struktur und elektronische und magnetische Eigenschaften - überprüft werden. Daher werden die verwendeten Charakterisierungsmethoden inklusive deren Messprinzipien kurz dargestellt.

\subsection{Metallorganische Aerosoldeposition}

Die untersuchte Dünnschichtprobe ist mittels metallorganischer Aerosoldeposition (MAD) [Moshnyaga99] hergestellt worden. Im Gegensatz zu vielen anderen Dünnschichtpräparationsmethoden findet die Probenherstellung hier nicht im Vakuum, sondern unter normalen Umgebungsbedingungen statt. Dadurch können stöchiometrische Sauerstoffleerstellen verhindert werden, die vor allem im untersuchten Manganatsystem eine große Rolle spielen, da die elektronischen Eigenschaften der Manganate vor allem durch Doppel- und Superaustausch über die Sauerstoffionen bestimmt sind [Gross12] (siehe Kapitel 4.1).

Abbildung 5.1 - Vereinfachter Aufbau der metallorganischen Aerosoldeposition. Ein Aerosol wird auf ein beheiztes Substrat gesprüht, auf dem sich schließlich der Dünnfilm bildet.

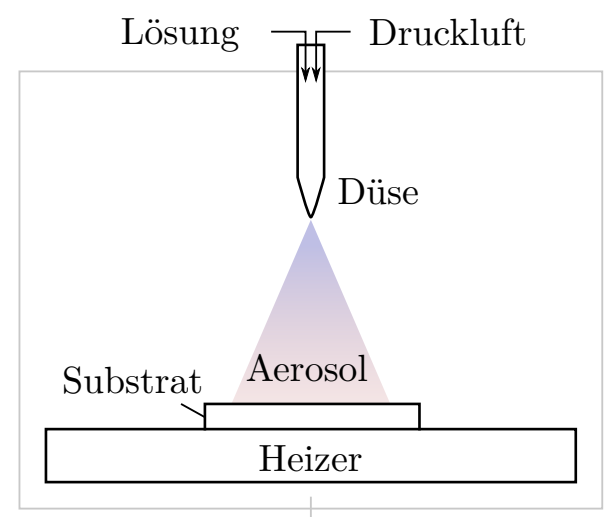


Dieses Verfahren ermöglicht es unter anderem, mit hohen Wachstumsraten von einigen Nanometern pro Minute zuverlässig oxidische epitaktische Dünnfilme herzustellen. Gleichzeitig ist der Aufwand der Schichtherstellung verhältnismäßig gering, da das Prinzip - siehe Abb. 5.1 - einfach ist. Zur Schichtherstellung werden Präkursoren aus metallorganischen Komplexen in Dimethylformamid gelöst. Diese Lösung wird dann mittels trockener Druckluft von zwei bis drei Bar durch eine pneumatische Düse auf ein beheiztes Substrat gesprüht. Die nötigen Temperaturen von etwa $650^{\circ} \mathrm{C}$ bis $1100{ }^{\circ} \mathrm{C}$ werden durch einen Heizwiderstand aus Siliziumkarbit realisiert. Diese hohen Temperaturen lösen eine pyrolytische Zersetzung aus, die die Trägermoleküle des Präkursors zu $\mathrm{CO}_{2}$ und $\mathrm{H}_{2} \mathrm{O}$ verbrennen lässt. Übrig bleiben die Metallionen der gewünschten Verbindung, die schließlich auf der Substratoberfläche adsorbieren.

Die Qualität der dünnen Filme, die mit der MAD hergestellt werden, ist außerordentlich hoch, vor allem wenn ein Präzisions-Spritzendosiersystem verwendet wird. So ist es zum Beispiel möglich, einzelne Atomlagen zu deponieren und so Schichtsysteme wie der Ruddlesden-Popper-SrO $\left(\mathrm{SrTiO}_{3}\right)_{n}$-Phase mit $n=4$ zu synthetisieren, die üblicherweise in der Natur nicht vorkommen [Jungbauer14].

Zur Qualitätsüberprüfung schon während der Probenherstellung wird das Wachstum in situ durch Ellipsometrie überwacht. Bei dieser Methode werden Polarisation und Phasenverschiebung eines Lichtstrahls gemessen, nachdem dieser auf der entstehenden Probenoberfläche reflektiert wurde. Ausführliche Erläuterungen zu diesem Verfahren findet man zum Beispiel in [Fujiwara07].

\subsection{SCHMELZSCHLEUDERN}

Zur Herstellung der metallischen Glasproben, die in dieser Arbeit untersucht werden, wird das Schmelzschleuderverfahren (engl. melt spinning) angewandt. Dabei werden zunächst die gewünschten Elemente in möglichst reiner Form im Lichtbogenofen zu einer Legierung verbunden. Anschließend wird diese - noch kristalline - Vorlegierung in der Schmelzschleuderanlage mit hohen Temperaturen von etwa $1000^{\circ} \mathrm{C}$ bis $2000^{\circ} \mathrm{C}$ in eine Schmelze gebracht und anschließend über eine Düse mit einem Öffnungsdurchmesser von etwa 2 mm auf ein Kup-

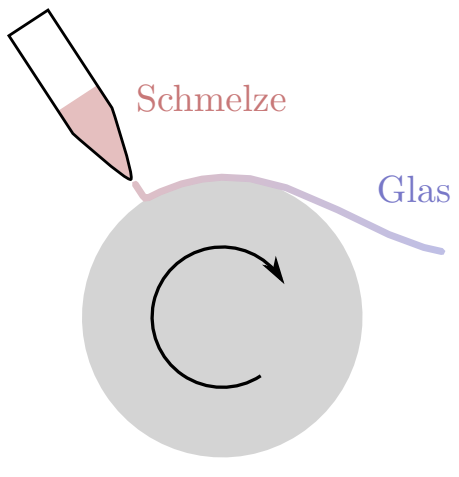

Abbildung 5.2 - Beim Schmelzschleuder-

verfahren wird eine Schmelze durch ein schnell rotierendes Kupferrad abgekühlt. 
ferrad gegossen. Hohe Rotationsgeschwindigkeiten des Kupferrades von etwa $40 \mathrm{~Hz}$ führen dazu, dass das Material extrem schnell abkühlt und anschließend als Band vom Rad geschleudert wird. Die Abkühlraten des Schmelzschleudern liegen bei $10^{5}$ bis $10^{6} \mathrm{~K} \mathrm{~s}^{-1}$. Die entstehenden Bänder haben üblicherweise eine Dicke von etwa $50 \mu \mathrm{m}$. Durch dieses Verfahren entstehen amorphe Proben, sofern dies für das jeweilige System möglich ist.

In dieser Arbeit wird der amorphe Zustand einer $\mathrm{Pd}_{77,5} \mathrm{Cu}_{6} \mathrm{Si}_{16,5}$-Zusammensetzung und sein kristallines Pendant untersucht. Die kristalline Vorlegierung könnte als Probe verwendet werden. Damit beide Proben vergleichbar bleiben, ist es jedoch sinnvoll, auf beiden Proben ein ähnliches Oberflächenprofil bzw. eine ähnliche Oberflächenrauigkeit zu haben. Eventuelle topographische Veränderungen des Schmelzschleuderverfahren müssen also beide Proben gleichermaßen betreffen. Daher werden die untersuchten kristallinen Varianten aus den amorphen Bändern hergestellt, indem sie im Vakuum bei etwa $50^{\circ} \mathrm{C}$ oberhalb der Kristallisationstemperatur für drei Stunden ausgelagert werden.

\subsection{Dynamische DifferenZKalorimetrie}

Die Untersuchung thermischer Eigenschaften der Glasproben wird mit der dynamischen (Leistungs-)Differenzkalorimetrie (engl. - differential scanning calorimetry, DSC) mit dem Gerät DSC7 der Firma PerkinElmer durchgeführt. Bei der DSC werden mit einer gewählten Heizrate gleichzeitig zwei Öfen erhitzt, die je eine Probe und eine Referenzprobe enthalten. Dabei wird die Differenz der elektrischen Leistungen beider Öfen gemessen. Bei exothermen Prozessen wird kurzzeitig weniger Leistung benötigt und das Messsignal wird kleiner, für Endotherme wird mehr Leistung benötigt. Zum Übergang von fest zu flüssiger Phase muss beispielsweise eine Schmelzenthalpie aufgebracht werden, die sich in einer DSC-Messung dann als lokales Maximum zeigt. Die gemessene Leistung $P$ ist mit der spezifischen Wärmekapazität

$$
c_{\mathrm{p}}=\frac{P}{m \dot{T}},
$$

der Masse $m$ der Probe und der Heizrate $\dot{T}$ verknüpft. Die Wärmekapazität ändert sich nahe der Glastemperatur, so dass neben Kristallisationsenthalpie und -temperatur auch die Glasübergangstemperatur $T_{\mathrm{g}}$ bestimmt werden kann.

Eine DSC-Messung wird zunächst mit Schmelztemperaturen und Schmelzenthalpien der Standardproben Indium und Zink kalibriert. Anschließend kann ei- 
ne Messung am Glas durchgeführt werden. Dazu wird Probenmaterial in einem Aluminiumtiegel verpresst, wobei als Referenzprobe ein leerer Tiegel verwendet wird, so dass man nur die Probe vermisst. Während die Differenzleistung gemessen wird, wird die Probe mit einer bestimmten Heizrate über die Kristallisationstemperatur erhitzt, abgekühlt und anschließend noch einmal erhitzt. Die Messkurve des zweiten Heizprozesses beschreibt daher die Wärmekapazität einer kristallinen Probe. Diese wird nun von der ersten Messung abgezogen, um ein deutlicheres Signal zu erhalten. Da die Wärmekapazitäten von Kristall und Glas ähnlich sind, ergibt dies eine Messkurve, die bis zum Glasübergang 0 ist. Ein Anstieg markiert dann den Glasübergang, auf den die Kristallisation folgt, die aufgrund der Abgabe von latenter Wärme ein tiefes Minimum zeigt (s. Kap. 5.6.2).

Die Bestimmung von $T_{\mathrm{g}}$ kann auf verschiedene Weise geschehen, hier wird die Doppeltangentenmethode angewandt, bei der zwei Tangenten an die Messkurve angelegt werden und deren Schnittpunkt bestimmt wird.

Das Probensystem $\mathrm{Pd}_{77,5} \mathrm{Cu}_{6} \mathrm{Si}_{16,5}$ hat eine Glasübergangstemperatur von $T_{\mathrm{g}} \approx$ $350{ }^{\circ} \mathrm{C}$ und eine Kristallisationstemperatur von $T_{\text {cryst }} \approx 430{ }^{\circ} \mathrm{C}$. Daher wird $550{ }^{\circ} \mathrm{C}$ als maximale Temperatur ausgewählt. Die Probenmasse liegt im Bereich von $10-$ $30 \mathrm{mg}$.

\subsection{StrukTURAnAlyse}

Die Strukturanalyse ist mit dem kommerziellen Röntgendiffraktometer D8 Advance von Bruker AXS [Bruker AXS01] durchgeführt worden. Dieses Gerät arbeitet in der Bragg-Brentano-Geometrie [Brentano24] (s. Abb. 5.3), bei der die Probe unter dem Winkel $\theta$ von Strahlung einer Röntgenröhre mit Kupferanode getroffen wird. Die Intensität der - durch Oberflächenrauigkeiten der Probe spekular gestreute - Röntgenstrahlung wird dann vom Detektor gemessen. Das Spektrum der Anode wird von den - durch Spin-Bahn-Kopplung getrennten - charakteristischen Wellenlängen $K_{\alpha 1}$ und $K_{\alpha 2}$ des Kupfers dominiert. Üblicherweise können diese Wellenlängen im Messspektrum nicht voneinander unterschieden werden, so dass der gewichtete Mittelwert $\bar{\lambda}=1 / 3\left(2 \lambda_{K_{\alpha 1}}+\lambda_{K_{\alpha 2}}\right)=1,54184 \AA$ verwendet wird.

Die gemessene Streuintensität mit dem Streuvektor $\vec{Q}=\overrightarrow{k^{\prime}}-\vec{k}$ und der Streudichte $\rho(\vec{r})$ ist [Gross12]

$$
I(\vec{Q}) \propto\left|\int_{V_{\mathrm{P}}} \rho(\vec{r}) \mathrm{e}^{-i \vec{Q} \cdot \vec{r}} \mathrm{~d} V\right|^{2}
$$


Dies entspricht der Fourier-Transformation der Streudichte $\rho$. Daher gilt: je größer der Streuvektor $\vec{Q}$ ist, desto kleinere Strukturen können aufgelöst werden.

\subsubsection{Röntgenbeugung}

Für kubische Strukturen mit Gitterkonstante $a$ und ausreichend großen Einfallswinkeln $\theta>5^{\circ}$ ergibt sich die Bragg-Bedingung und es können mittels Röntgenbeugung Netzebenenabstände $d_{h k l}=a / \sqrt{h^{2}+k^{2}+l^{2}}$ mit den Millerschen Indizes $h, k, l$ bestimmt werden [Miller39, Bragg13, Friedrich13]:

$$
\sin \theta=\frac{n \lambda}{2 d_{h k l}},
$$

wobei $n$ einer ganzzahlige Ordnung entspricht.

\subsubsection{RÖNTGENREFLEKTOMETRIE}

Wählt man für die Röntgenstrahlung kleine Einfallswinkel $\theta \leq 5^{\circ}$, wird $\vec{Q}$ im Vergleich zum reziproken Gittervektor klein. Dadurch verliert man das Aufösungsvermögen für atomare Abstände. Die Röntgenstrahlen reflektieren hier jedoch an Grenzflächen unterschiedlicher Brechungsindizes. Das gemessene Signal zeigt daher winkelabhängige Interferenzen, deren Periode Informationen der Schichtdicke und deren Abfall Informationen zur Grenzflächenrauigkeit enthält

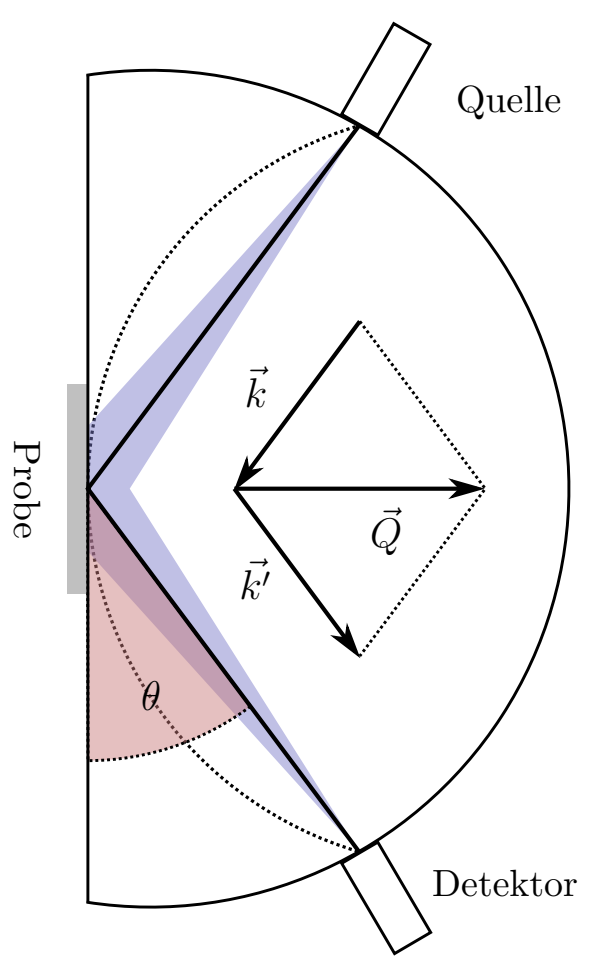

Abbildung $\mathbf{5 . 3}-$ Vereinfachter Aufbau der RöntgenStrukturanalyse in BraggBrentano-Geometrie. [Daillant08].

\subsubsection{Streunng An Amorphen Systemen}

Die Struktur amorpher Materialien ist - wie beschrieben - nicht periodisch. Das Fehlen von Periodizitäten wie Netzebenen führt dazu, dass keine scharfen Beugungsmaxima beobachtet werden. Die Nahordnung führt allerdings trotzdem zu Beugungseffekten und es ergeben sich - je nach System - ein breites Maximum oder mehrere breite Maxima. Grund für die Breite ist die zufällige Abstandsverteilung 
der Atome und der daraus resultierenden breiteren Form der radialen Verteilungsfunktion. Dadurch ist es auch möglich, mit einer Fourier-Analyse die mittlere Zahl der Atome zu bestimmen, die einen bestimmten Abstand zu einem Atom haben [Charles02].

\subsection{Transportmessung \& Magnetometrie}

Wie beschrieben sind die magnetischen und elektrischen Eigenschaften eines Manganats durch dessen Dotierung bestimmt. Daher wird die Probe hinsichtlich dieser Eigenschaften untersucht. Zum einen dient das kommerzielle Physical Property Measurement System (PPMS) dazu, mittels Vier-Punkt-Widerstandsmessungen den spezifischen Widerstand in Abhängigkeit von der Temperatur der Probe zu bestimmen, zum anderen dient das Magnetic Property Measurement System (MPMS) dazu, die Magnetisierung einer Probe in Abhängigkeit von der Temperatur und/oder vom Magnetfeld zu bestimmen. Beide Geräte sind von der Firma Quantum Design und können Temperaturbereiche von etwa $1,9 \mathrm{~K}$ bis $400 \mathrm{~K}$ und Magnetfelder von $9 \mathrm{~T}$ (PPMS) bzw. $5 \mathrm{~T}$ (MPMS) erreichen.

\subsection{ERGEBNisse DeR CharaKterisierung}

Im Vordergrund dieser Arbeit stehen Reibungsmessungen sowie deren Interpretationen. Genaue Informationen über die untersuchten Proben sind daher wichtig. Um sich in den folgenden Kapiteln auf die Reibung konzentrieren zu können, sollen die Ergebnisse der Charakterisierungsmessungen bereits an dieser Stelle gezeigt werden.

\subsection{1 $\mathrm{LA}_{0,6} \mathrm{SR}_{0,4} \mathrm{MNO}_{3}$}

Zunächst werden einige grundlegende magnetische, elektrische und strukturelle Eigenschaften der LSMO-Probe gezeigt. Die LSMO-Probe inklusive der Charakterisierungsmessungen sind von Danny Schwarzbach im Rahmen seiner Masterarbeit am I. Physikalischen Institut der Universität Göttingen hergestellt worden [Schwarzbac16]. 


\section{ELEKTRISCHER WIDERSTAND}

Der elektrische Widerstand in Abhängigkeit von der Temperatur ist in Abbildung 5.4 dargestellt. Die Metall-Metall-Übergangstemperatur wird dabei mit der Funktion $\frac{1}{R} \frac{\mathrm{d} R}{\mathrm{~d} T}$ bestimmt. Diese Funktion zeigt an der Übergangstemperatur - sofern vorhanden - ein Maximum, das hier bei $T_{\mathrm{MM}}=352 \mathrm{~K}$ liegt. Das entspricht nach dem Phasendiagramm (s. Kap. 4.1.5) der erwarteten Temperatur.

Abbildung 5.4 - Der Widerstand in Abhängigkeit von der Temperatur der LSMO-Probe. Die Funktion $\frac{1}{R} \frac{d R}{d T}$ zeigt bei $T_{M M}=352 \mathrm{~K}$ ein Maximum.

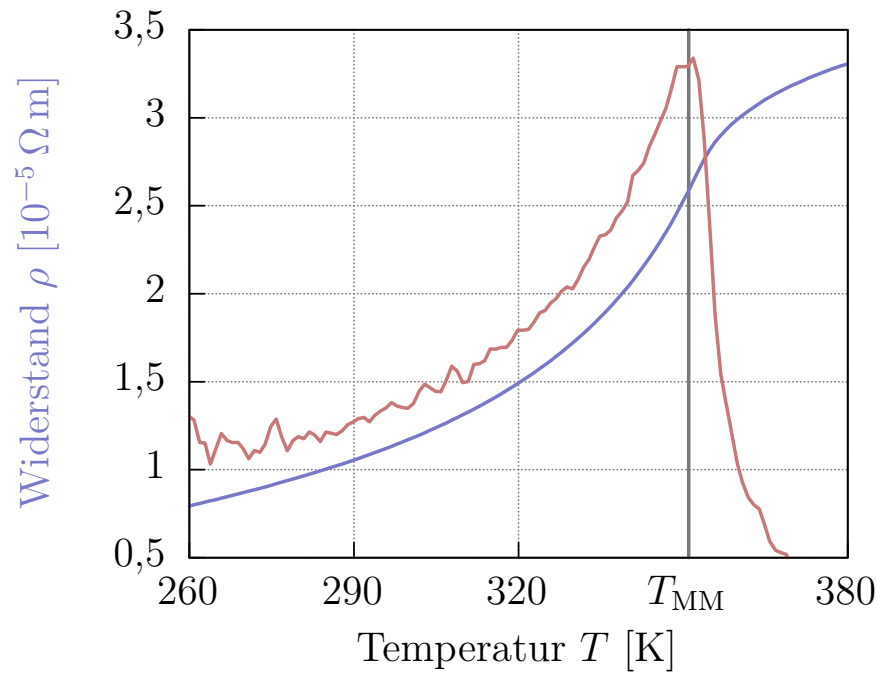

\section{Magnetisierung}

Abbildung 5.5 zeigt die magnetischen Eigenschaften der LSMO-Probe. Zum einen wird in a) die Sättigungsmagnetisierung bei $10 \mathrm{~K}$ bestimmt, indem das angelegte Feld von $-2 \mathrm{~T}$ bis $2 \mathrm{~T}$ variiert wird. Sie liegt bei 3,6 $\mu_{\mathrm{B}} / \mathrm{Mn}$, zum anderen zeigt b) die Bestimmung der Curie-Temperatur. Sie wird über das Maximum der Funktion $\frac{1}{M} \frac{\mathrm{d} M}{\mathrm{~d} T}$ bestimmt und liegt bei $T_{\mathrm{C}}=362 \mathrm{~K}$. Die Sättigungsmagnetisierung ist entscheidend, da neben dem Rumpfspin auch die e $\mathrm{g}$-Manganionspins zur Magnetisierung beitragen und es gilt für die Gesamtmagnetisierung des $\mathrm{La}_{1-x} \mathrm{Sr}_{x} \mathrm{MnO}_{3}-$ Systems

$$
M=(4-x) \mu_{\mathrm{B}}
$$

Die gemessene Magnetisierung zeigt also, dass die Probe die korrekte Dotierung aufweist. 
a) $M(H)$-Messung bei $10 \mathrm{~K}$

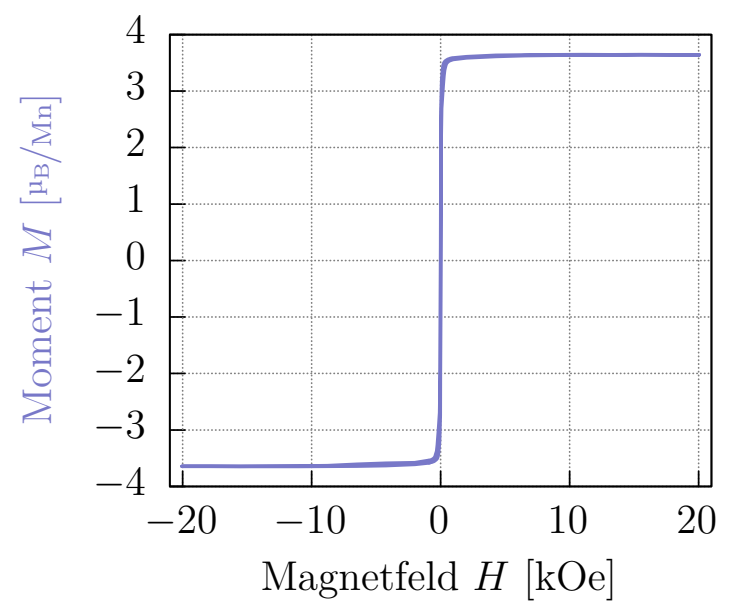

a) $M(T)$-Messung bei $100 \mathrm{mT}$

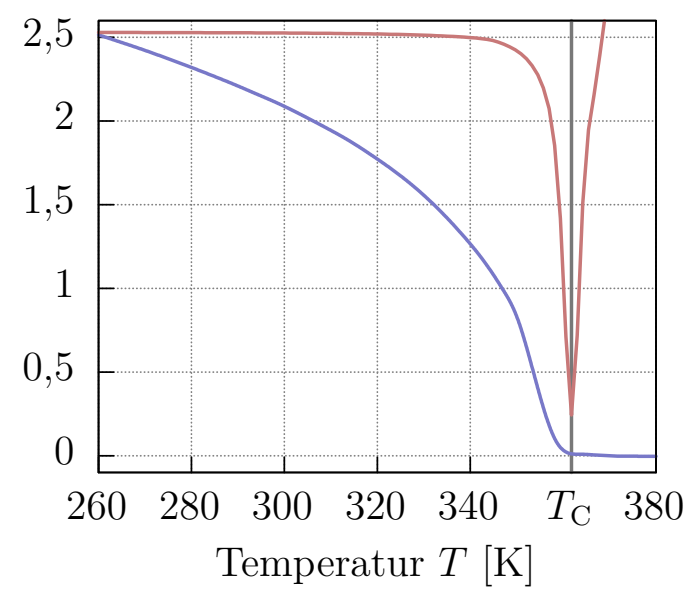

Abbildung 5.5 - Magnetische Eigenschaften der LSMO-Probe. a) Die Sättigungsmagnetisierung bei $10 \mathrm{~K}$ liegt bei 3,6 $\mathrm{\mu}_{B} / \mathrm{Mn}$. b) Die Curie-Temperatur wird über das Maximum der Funktion $\frac{1}{M} \frac{d M}{d T}$ bestimmt und liegt bei $T_{C}=362 \mathrm{~K}$.

\section{RÖNTGENMESSUNGEN}

Abbildung 5.6 zeigt die Röntgenmessung an der LSMO-Probe. Die Simulation des Kleinwinkelspektrums mit dem Programm ReMagX [Macke14] liefert zum einen die Oberflächenrauigkeit $\sigma_{\mathrm{RMS}}=5,5 \AA$, die mit STM-Messungen übereinstimmt (nicht gezeigt), zum anderen die Schichtdicke von $d=40,52 \mathrm{~nm}$. Das Weitwinkelspektrum liefert die pseudokubische out-of-plane Gitterkonstante $a=(3,830 \pm 0,002) \AA$.

\subsection{2 $\mathrm{PD}_{77,5} \mathrm{Cu}_{6} \mathrm{SI}_{16,5}$}

Um sicher zu gehen, dass die $\mathrm{Pd}_{77,5} \mathrm{Cu}_{6} \mathrm{Si}_{16,5}$-Proben amorph bzw. kristallin sind, sind Röntgenspektren aufgenommen und DSC-Messungen durchgeführt worden. Abbildung 5.7 zeigt die DSC-Messung der amorphen Probe. Das eingesetzte Bild zeigt den Glasübergang bei der Temperatur $T_{\mathrm{g}} \approx 625 \mathrm{~K}$. Die DSC-Messung der kristallinen Probe (nicht gezeigt) zeigt diesen Übergang - wie erwartet - nicht.

Auch die Röntgenspektren in Abbildung 5.8 zeigen die unterschiedlichen Formen der Probe. Das amorphe Material zeigt zwei typisch breite Maxima. Die polykristalline Variante zeigt viele Peaks. 
a) XRR-Messung

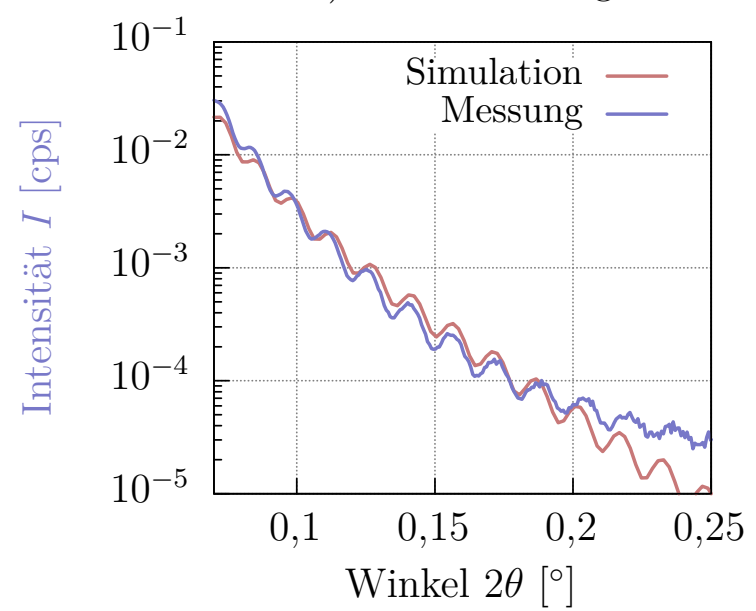

b) XRD-Messung

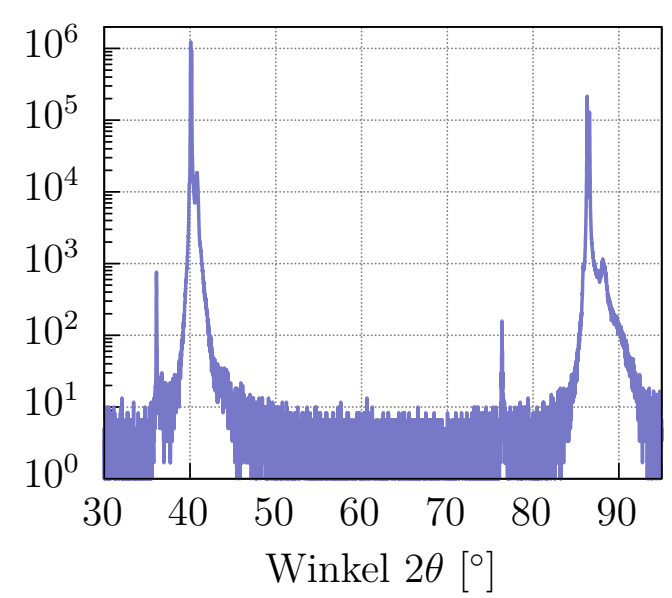

Abbildung 5.6 - Die Röntgenmessung der LSMO-Probe. a) Die Simulation des Kleinwinkelspektrums liefert die Oberflächenrauigkeit $\sigma_{R M S}=5,5 \AA$ und die Schichtdicke $d=40,52 \mathrm{~nm}$. b) Das Weitwinkelspektrum liefert die Gitterkonstante $a=$ $(3,830 \pm 0,002) \AA$.

Abbildung 5.7 - DSC-Messung zur Bestimmung der Glasübergangstemperatur.

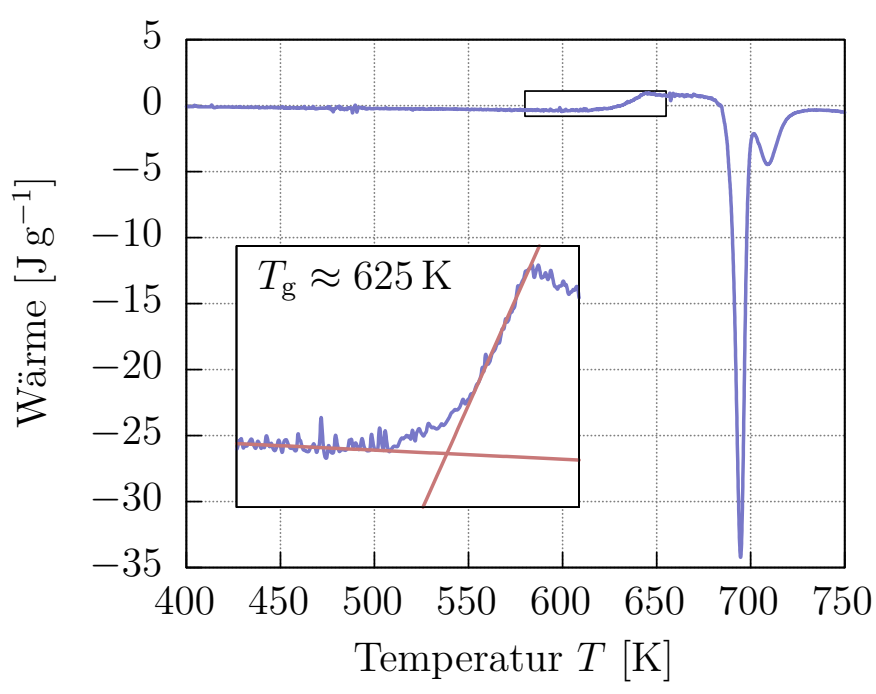


a) amorphes $\mathrm{Pd}_{77,5} \mathrm{Cu}_{6} \mathrm{Si}_{16,5}$

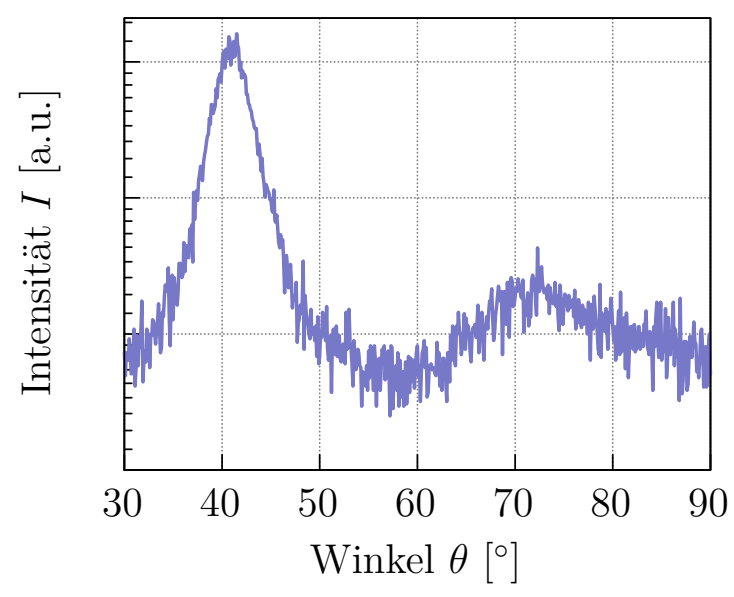

b) kristallines $\mathrm{Pd}_{77,5} \mathrm{Cu}_{6} \mathrm{Si}_{16,5}$

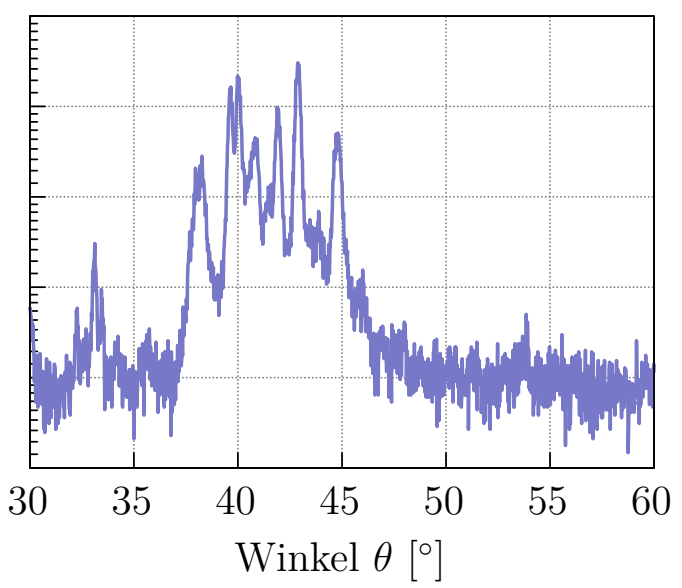

Abbildung 5.8 - Die Röntgenmessung der $\mathrm{Pd}_{77,5} \mathrm{Cu}_{6} \mathrm{Si}_{16,5}$-Probe. a) Die amorphe Probe zeigt zwei breite Maxima. b) Die polykristalline Variante hingegen zeigt viele kristalline Peaks. 


\section{SONOLUBRICATION}

In Kapitel 2.3.1 ist gezeigt worden, dass eine mechanische Anregung der Blattfeder im Prandt-Tomlinson-Modell zu einer linearen Reibungsabnahme führt. Allerdings wird in diesem eindimensionalen Modell - und so auch in den Arbeiten [Socoliuc06, Gnecco08, Iizuka09, Fajardo14] - vernachlässigt, dass die Blattfeder nichtlineare Kräfte erfährt (vgl. Kap. 3.2.1). Die Arbeiten von Dinelli et al. [Dinelli97] und Scherer et al. [Scherer99] zeigen, dass die Reibung bei Scanwegen von mehreren hundert Nanometern und Blattfederamplituden unter einem Nanometer nicht-linear mit der Amplitude abnimmt. In beiden Arbeiten wird die starke Reibungsminderung als ein Ablösen der Blattfeder von der Probenoberfläche interpretiert. Intuitiv ist klar, dass die Blattfeder bei ausreichend großen Amplituden den Kontakt zur Probe verlieren kann und dadurch für einen gewissen Zeitraum keine Reibung gemessen wird, bis die Blattfeder wieder aufsetzt. Allerdings wird in beiden Arbeiten auch gezeigt, dass die Reibung schon vor solch einem Abheben bereits um mehr als $40 \%$ reduziert sein kann. Es wird vermutet, dass ein Wasserfilm auf der Probe die Ursache der Verminderung ist. Dieser soll unter Anregung aufgrund von hydrodynamischen Eigenschaften zu einem zusätzlichen Abheben führen.

Im Folgenden wird eine analytische Beschreibung der Reibungsverminderung mit akustischer Anregung - der sogenannten Sonolubrication, also akustischen Schmierung - anhand von Messergebnissen entwickelt. Dazu wurden die Probensysteme $\mathrm{La}_{1-x} \mathrm{Sr}_{x} \mathrm{MnO}_{3}, \mathrm{Pd}_{77,5} \mathrm{Cu}_{6} \mathrm{Si}_{16,5}$ und Silizium untersucht (s. Kap. 4). Im Folgenden werden Ergebnisse präsentiert, die teilweise in [Pfahl18] veröffentlicht sind. Sie sind dementsprechend gekennzeichnet.

\subsection{ReIBUngShysteresen}

Um die folgenden Ergebnisse diskutieren zu können, werden zunächst neue Parameter eingeführt. Diese beinhalten Daten aus Reibungskraftmessungen, die mittels FFM-Methode aufgenommen worden sind. In Kapitel 3.2.2 ist dieses Verfahren erklärt worden. Basis dieser Methode bildet das Aufnehmen von Reibungshysteresen. 


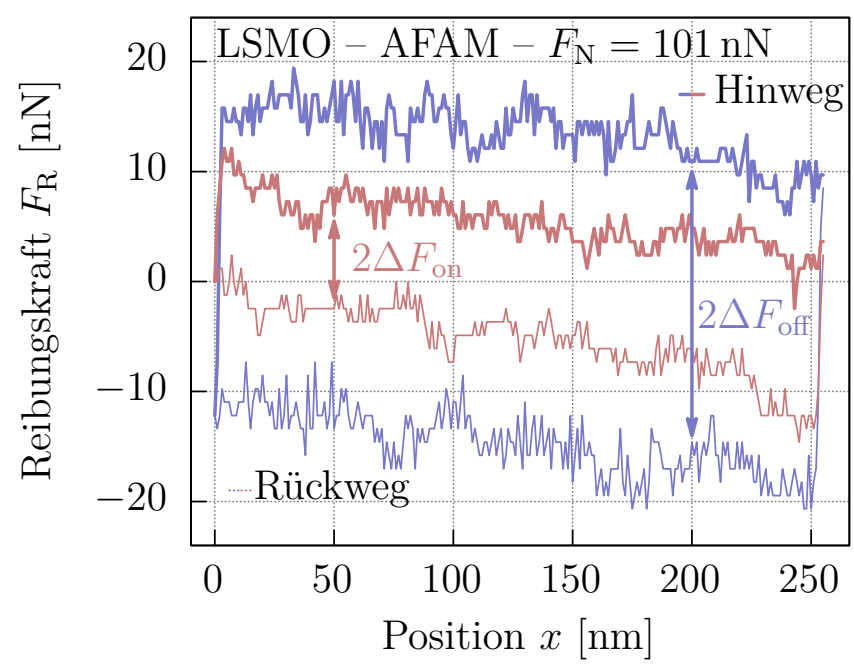

Abbildung 6.1 - Zwei Reibungshysteresen bei gleicher Normalkraft $F_{N}=101 \mathrm{nN}$. Die mittlere Reibungskraft ohne Anregung $\Delta F_{\text {off }}$ ist etwa doppelt so groß wie $\Delta F_{\text {on }}$ mit externer $A n$ regung mit $A_{D}=0,2 \mathrm{~V}$. Nach [Pfahl18].

Abbildung 6.1 zeigt beispielhaft zwei solcher Reibungshysteresen auf der LSMOProbe. Die blaue Kurve zeigt eine Messung ohne, die rote mit externer Anregung. Um nun die durchschnittliche Reibungskraft $\Delta F$ aus einer einzelnen Reibungshysterese zu extrahieren, wird zunächst die Reibungskraft $\bar{F}_{x}=0,5\left(F_{x \text {,hin }}-F_{x \text {,rück }}\right)$ an jeder vermessenen Position $x$ bestimmt, dabei werden die Bereiche der Richtungsänderungen, in denen sich das Vorzeichen der Torsion ändert, nicht berücksichtigt, da in diesen Bereichen keine sinnvolle Reibungskraft extrahiert werden kann. Anschließend wird das arithmetische Mittel aller $\bar{F}_{x}$-Werte berechnet, so dass pro Reibungshysterese ein einzelner Messwert der durchschnittlichen Reibungskraft $\Delta F=\sum_{x}^{n}\left(\bar{F}_{x}\right) / n$ entsteht.

Um Messfehler zu minimieren, werden mehrere Hysteresen aufgenommen, wobei nach jeder Messung in $x$-Richtung die $y$-Richtung leicht verändert wird. Daher bezeichnet man die $x$-Richtung auch als schnelle, die $y$-Richtung als langsame Scanrichtung. Dadurch entsteht eine zweidimensionale Reibungskarte, die im Folgenden $x=256 \mathrm{~nm}$ lang und $y=24 \mathrm{~nm}$ breit ist (s. Abb. 6.2). Die gezeigten Messwerte sind daher immer eine Mittlung von etwa 6000 Werten.

Um Fehler durch Spitzenveränderung deutlich zu verkleinern und so $\Delta F_{\text {on }}$ und $\Delta F_{\text {off }}$ besser miteinander vergleichen zu können, werden die Daten im sogenannten Interleave-Modus (engl. verschachtelt) aufgenommen. Dazu wird erst eine Hysterese ohne Anregung und direkt anschließend an der gleichen Position y eine Hysterese mit Anregung aufgenommen. Dadurch verdoppelt sich die Messzeit einer Aufnahme, die aber dadurch je eine Reibungskarte mit und ohne Anregung enthält.

Abbildung 6.2 zeigt beispielhaft das Höhenprofil (rot) und die Reibungskarten (blau) einer Siliziumprobe. Die Reibungskarten sind in gleicher normierter Farbska- 

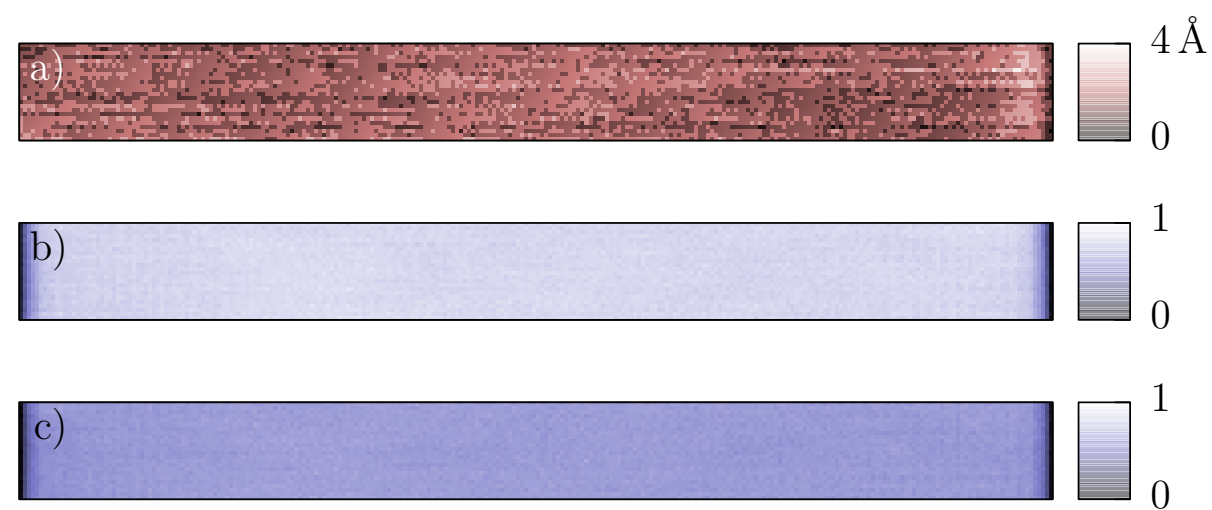

\begin{abstract}
Abbildung 6.2 - Messung auf Silizium mit $28 \times 256 \mathrm{~nm}^{2}$. Im Interleave-Modus werden gleichzeitig a) das Höhenprofil, b) die Reibungskarte ohne und c) die Reibungskarte mit Anregung aufgenommen. Die Reibungskarten sind hier normiert, die dunklere Farbe in c) zeigt, dass die Reibung unter Anregung gemindert ist. Die dunklen Bereiche am Rand der Reibungskarten zeigen den Vorzeichenwechsel der Torsion.
\end{abstract}

la dargestellt. Hellere Werte entsprechen einer höheren Reibung. Die Reibungskarte ohne Anregung b) ist im Vergleich zur Reibungskarte mit Anregung c) heller.

Abbildung 6.1 und Abbildung 6.2 zeigen bereits, dass die Reibung unter Anregung reduziert ist. Im Folgenden beschreibt der Parameter $\Delta F_{\text {off }}$ den gemessenen Mittelwert der Reibungskraft ohne, der Parameter $\Delta F_{\text {on }}$ den mit externer Anregung. Des Weiteren wird die Differenz dieser Werte als

$$
\Delta F=\Delta F_{\text {off }}-\Delta F_{\text {on }}
$$

bezeichnet. Dieser Wert gibt also an, um wie viel Newton die Reibungskraft unter Anregung gemindert wird. Die externe Anregung wird quantifiziert durch die gemessene Amplitude $A_{\mathrm{C}}$ der Blattfeder. Sie ist abhängig von Kontakteigenschaften und der anregenden Amplitude. Diese wird - wie beschrieben - im AFAM-Modus durch einen Schallwandler unter der Probe, im UAFM-Modus mit dem Schallwandler des Tapping-Modus realisiert. Die Spannung, die den jeweiligen Schallwandler treibt, wird im Folgenden als Amplitude $A_{\mathrm{D}}$ bezeichnet. Geht die Amplitude der Blattfeder gegen 0 , geht $\Delta F_{\text {on }}$ gegen $\Delta F_{\text {off }}$, so dass $\Delta F=0$. Trägt man $\Delta F$ gegen die Amplitude auf, entspringt die Messkurve daher grundsätzlich dem Ursprung. Für ausreichend große Anregungsamplituden geht $\Delta F_{\text {on }}$ gegen null, so dass $\Delta F$ sättigt und gegen den Wert $\Delta F_{\text {off }}$ strebt. 


\subsection{AFAM vs. UAFM}

Zunächst soll untersucht werden, ob AFAM- und UAFM-Modus qualitativ identische Messergebnisse erzeugen und dadurch vergleichbar bleiben. Abbildung 6.3 a) zeigt zwei Kontaktresonanzkurven mit der Anregungsamplitude $A_{\mathrm{D}}=2,5 \mathrm{~V}$. Es ist zu erkennen, dass Amplitude, Frequenz und Qualitätsfaktor von der Normalkraft $F_{\mathrm{N}}$ abhängen. Je größer die Normalkraft ist, desto größer wird die Kontaktsteifigkeit und somit auch die Kontaktfrequenz (vgl. Kap. 3.3.3).

a) AFAM-Resonanzkurven

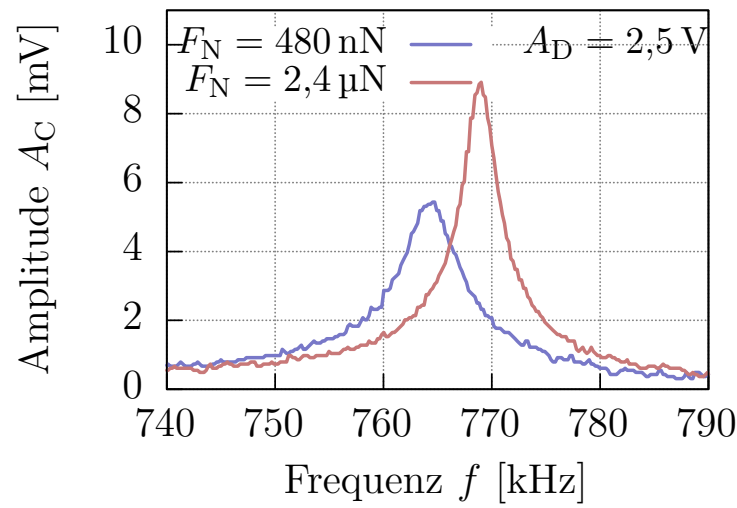

b) UAFM-Resonanzkurven

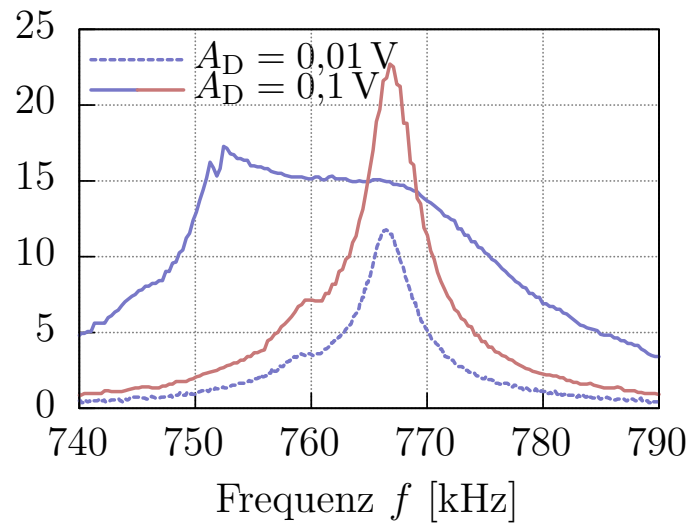

Abbildung 6.3 - Resonanzkurven der ersten Biegemode. Die Auflagekraft ist $F_{N}=$ $480 \mathrm{nN}$ (blau) bzw. $F_{N}=2,4 \mu \mathrm{N}$ (rot). Die Federkonstante beträgt $48 \mathrm{~N} \mathrm{~m}^{-1}$. a) Eine höhere Auflagekraft führt zu höheren Kontaktfrequenzen und höherem Q-Wert.

Abbildung 6.3 b) zeigt Resonanzkurven im UAFM-Modus. Zunächst zeigt sich, dass im UAFM-Modus deutlich kleinere Anregungsamplituden im Millivoltbereich schon zu größeren Blattfederamplituden führen. Das führt auch dazu, dass zu hohe Anregungsamplituden zu unkontrolliertem Verhalten der Blattfeder führen können. Die blaue durchgezogene Resonanzkurve zeigt einen solchen Fall. Die Anregungsamplitude von $0,1 \mathrm{~V}$ bei einer Auflagekraft von $F_{\mathrm{N}}=480 \mathrm{nN}$ ist so groß, dass die Blattfeder sich zeitweise von der Probenoberfläche löst, so dass die AFM-Rückkopplung eingreift und die Blattfeder absenkt. Dies ist daran zu erkennen, dass sich bei der Frequenz $f \approx 753 \mathrm{kHz}$ - also im Maximum der Kurve - das gemessene Signal des Auslenkungsfehlers (engl. Deflection Error) ändert, woraufhin die Rückkopplung den z-Piezo nachregelt. Das wiederum wird im Signal des Höhensensors sichtbar. Durch das Eingreifen der Rückkopplung ändert sich die Resonanzkurve unstetig, da durch das plötzliche Herabsenken der Spitze die Auflagekraft vergrößert wird. 
Die rote Kurve zeigt, dass bei einer höheren Auflagekraft von $F_{\mathrm{N}}=2,4 \mu \mathrm{N}$ wieder eine Lorentzkurve entsteht.

Das Auftreten dieses unkontrollierten Verhaltens kann mit der Überwindung einer Haftreibung in Verbindung gebracht werden. Im Folgenden wird ein solches Verhalten allerdings vermieden. Dies ist am einfachsten umzusetzen, indem die Anregungsamplitude verringert wird. Die gestrichelte blaue Linie in Abbildung 6.3 b) zeigt eine Kontaktresonanzkurve mit identischer Auflagekraft wie im chaotischen Fall, jedoch mit einer geringeren Anregungsamplitude von nur $10 \mathrm{mV}$. Dadurch wird die Lorentzform wieder hergestellt.

Ein weiteres Merkmal der Resonanzkurven im UAFM-Modus sind zusätzliche Peaks. In Abbildung $6.3 \mathrm{~b}$ ) tritt dies beispielsweise bei $f \approx 760 \mathrm{kHz}$ auf. Diese Resonanzen kommen nicht vom Kontakt, sondern sind dem Blattfederhalter zuzuschreiben, da sie in AFAM-Resonanzen nicht auftreten.

a) AFAM-Anregung

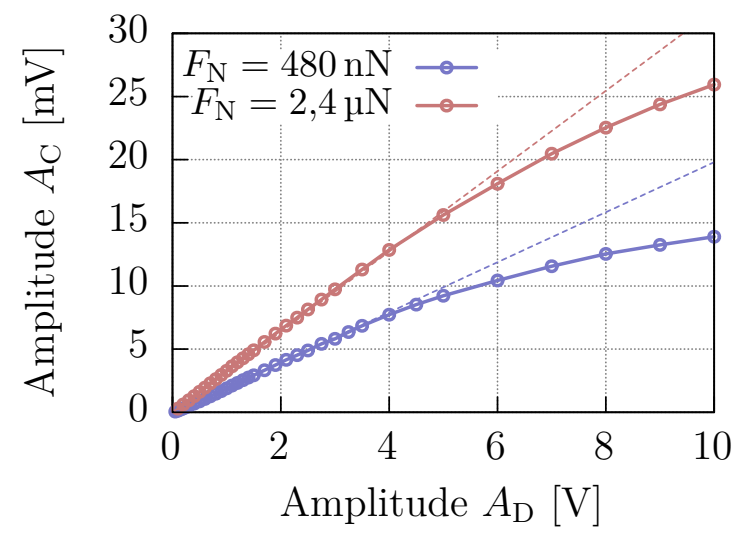

b) UAFM-Anregung

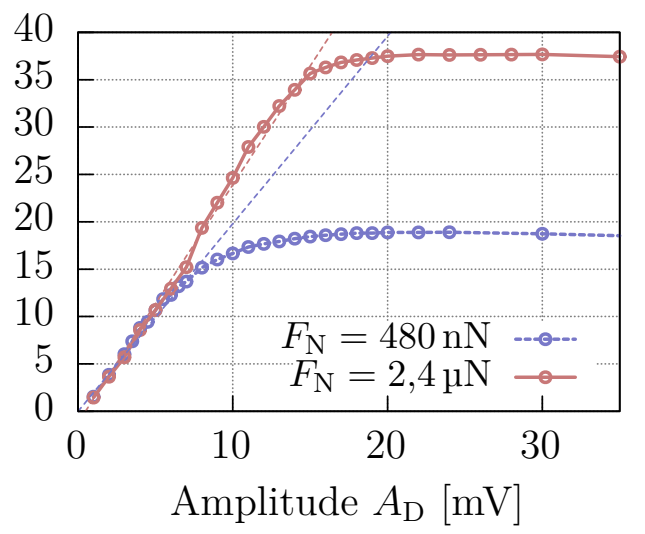

Abbildung 6.4 - Blattfederamplitude $A_{C}$ als Funktion der Anregungsamplitude $A_{D}$ im a) AFAM-Modus und im b) UAFM-Modus. In beiden Modi wird zunächst eine lineare Abhängigkeit festgestellt. Nicht-lineare oder sättigende Bereiche werden im Experiment vermieden.

Neben der Auflagekraft hat natürlich auch die Anregungsamplitude Einfluss auf die resultierende Blattfederamplitude. Abbildung 6.4 zeigt das Verhalten der Blattfederamplitude $A_{\mathrm{C}}$ in Abhängigkeit von der anregenden Amplitude $A_{\mathrm{D}}$ im AFAMund UAFM-Modus für zwei verschiedene Auflagekräfte. Zunächst kann man erkennen, dass bis zu einer gewissen Anregungsamplitude in beiden Modi ein lineares Verhalten vorliegt. Bei höheren Anregungen verringert sich die Steigung. Im 


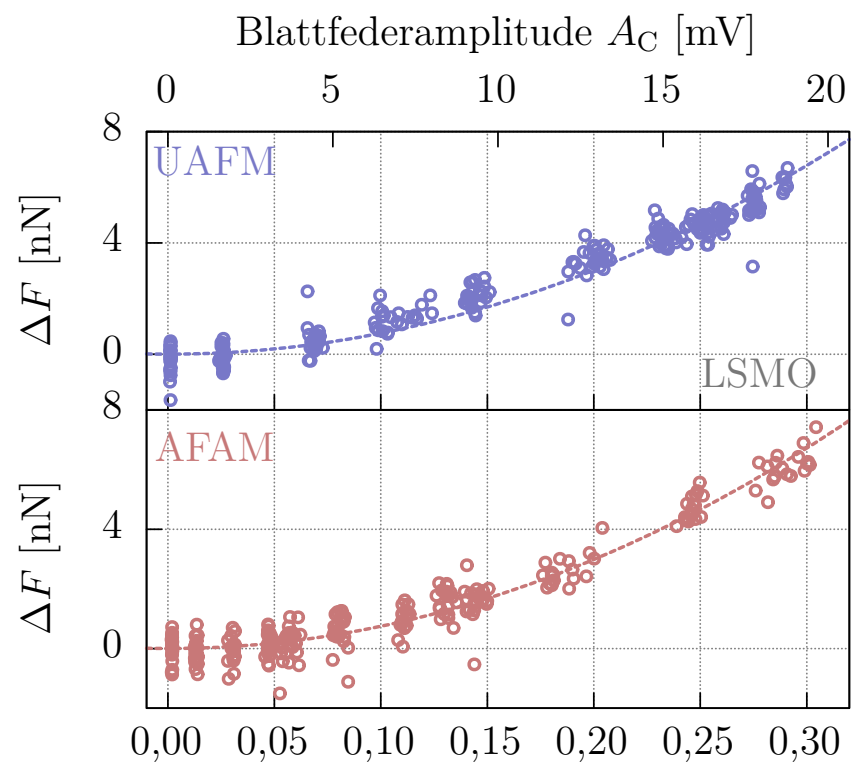

Abbildung 6.5 - Die Differenz der Reibungskräfte $\Delta F$ in $A b$ hängigkeit von der Blattfederamplitude $A_{C}$ im UAFM- und AFAM-Modus bei der Normalkraft $F_{N}=84 \mathrm{nN}$. Die quadratischen Fits (gestrichelte Linien) an $\Delta F=b A_{B}^{2}$ liefern $b_{A F A M}=$ $(74,7 \pm 0,9) \mathrm{GPa}$ und $b_{U A F M}=$ $(75,4 \pm 0,7) \mathrm{GPa}$.

UAFM-Modus stellt sich sogar eine Sättigung mit anschließender minimaler Abnahme der Blattfederamplitude ein.

Weiterhin ist zu erkennen, dass die maximale Anregungsamplitude $A_{\mathrm{D}}=10 \mathrm{~V}$ im AFAM-Modus ähnliche Blattfederamplituden auslöst wie $A_{\mathrm{D}} \leq 10 \mathrm{mV}$ im UAFMModus.

Zusammenfassend kann man sagen, dass in beiden Modi ähnliche Resonanzkurven beobachtet werden, sofern chaotisches und nicht-lineares Verhalten vermieden werden. Die Kurven des AFAM-Modus sind frei von Nebenpeaks, im UAFM-Modus hingegen können höhere Amplituden erreicht werden. Die Blattfederamplitude ist dabei abhängig von der anregenden Frequenz $f$, der Anregungsamplitude $A_{\mathrm{D}}$ und der Auflagekraft $F_{\mathrm{N}}$.

Abbildung 6.5 zeigt je eine Messung der Reibungskraftdifferenz $\Delta F$ im AFAMund UAFM-Modus bei konstanter Normalkraft $F_{\mathrm{N}}=84 \mathrm{nN}$ und konstanter Kontaktresonanzfrequenz $f=396 \mathrm{kHz}$. Die Blattfederamplitude wird hier variiert, indem die Anregungsamplitude geändert wird. Die gestrichelten Linien sind quadratische Anpassungen an die Funktion $\Delta F=b A_{\mathrm{B}}^{2}$. Die ermittelten Werte sind $b_{\mathrm{AFAM}}=(74,7 \pm 0,9) \mathrm{GPa}$ und $b_{\mathrm{UAFM}}=(75,4 \pm 0,7) \mathrm{GPa}$, so dass man im Folgenden davon ausgehen kann, dass AFAM- und UAFM-Modus zu identischen Messergebnissen führen. 


\subsection{VARiation der Blattfederamplitude}

Abbildung 6.6 zeigt die Reibungsdifferenz $\Delta F$ in Abhängigkeit von der Blattfederamplitude $A_{\mathrm{C}}$ für zehn verschiedene Auflagekräfte von 12 bis $253 \mathrm{nN}$ im AFAMModus bzw. von 16 bis $253 \mathrm{nN}$ im UAFM-Modus. Auch hier ist die Amplitude der Blattfeder über die Anregungsamplitude verändert worden.

Wie bereits erwähnt werden im AFAM-Modus im Vergleich zum UAFM-Modus geringere Blattfederamplituden erreicht. Daher endet die Messkurve im AFAMModus bereits bei $A_{\mathrm{C}} \approx 3,5 \AA$. Trotzdem ist auch hier zu erkennen, dass $\Delta F$ in beiden Modi die gleiche Abhängigkeit von $A_{\mathrm{C}}$ zeigt. Zusätzlich ist zu erkennen, dass die Auflagekraft offenbar keinen Einfluss auf die Abhängigkeit hat. Dies ist ungewöhnlich für Reibungsmessungen, kann aber verstanden werden, wenn man davon ausgeht, dass $\Delta F_{\text {off }}$ und $\Delta F_{\text {on }}$ die gleiche Reibungskrafterhöhung mit höherer Normalkraft erfahren, so dass die Differenz $\Delta F$ bei fester Anregungsamplitude konstant bleibt.
Abbildung 6.6 - Die Reibungs- kraftdifferenz $\Delta F$ in Abhängig- keit von der Blattfederamplitu- de $A_{C}$ im UAFM und AFAM- Modus, jeweils für zehn ver- schiedene Normalkräfte. Nach [Pfahl18].

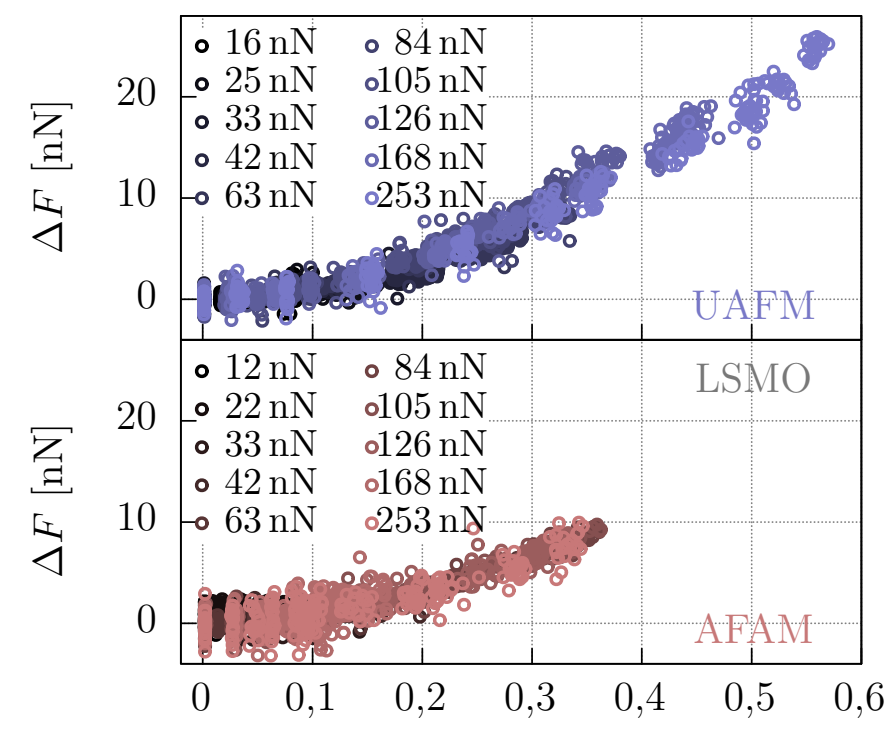

Die Amplitude der Blattfeder kann auch über die Änderung der Anregungsfrequenz bei konstanter Normalkraft und konstanter Anregungsamplitude geändert werden - man spricht von einem Frequenz-Sweep ( $f$-Sweep). Dabei sweept man sinnvollerweise über die Kontaktresonanzfrequenz, um eine hohe Änderung der Blattfederamplitude zu erreichen. Die andere Möglichkeit, die Blattfederamplitude $\mathrm{zu}$ variieren, ist der Normalkraft-Sweep $\left(F_{\mathrm{N}}\right.$-Sweep), bei dem Frequenz und Anregungsamplitude konstant gehalten werden, während die Normalkraft geändert wird. Abbildung 6.7 zeigt die Reibungsdifferenz $\Delta F$ in Abhängigkeit von der Blattfederamplitude $A_{\mathrm{C}}$ im AFAM- und UAFM-Modus für je zwei Normalkräfte. Zusätzlich 


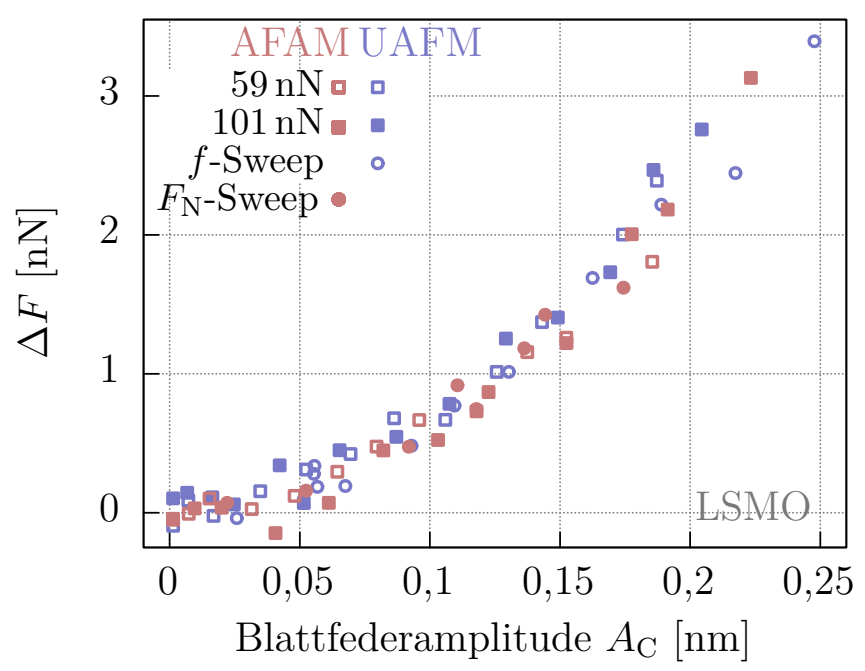

Abbildung 6.7 - Die Differenz der Reibung $\Delta F$ in Abhängigkeit von der Blattfederamplitude $A_{C}$ im AFAM- (rot) und UAFM- Modus (blau) der LSMO-Probe. $A_{C}$ ist geändert worden durch Anregungsamplitudensweeps bei $F_{N}=$ $59 \mathrm{nN}$ (offenes Quadrat) bzw. $F_{N}=101 \mathrm{nN}$ (volles Quadrat), Frequenz- und Normalkraftsweeps (Kreise). Nach [Pfahl18].

ist ein Frequenz-Sweep im UAFM-Modus und ein Normalkraft-Sweep im AFAMModus eingetragen. Auch hier führen alle Varianten zur gleichen quadratischen Abhängigkeit.

Die Differenz der Reibungskräfte $\Delta F$ ist daher eine Funktion der Blattfederamplitude $A_{\mathrm{C}}$. Diese ist zwar von verschiedenen Parametern wie Normalkraft $F_{\mathrm{N}}$, Anregungsamplitude $A_{\mathrm{D}}$ und Anregungsfrequenz $f$ abhängig, nominal ist die Reibungsminderung aber nur von der tatsächlichen Amplitude der Blattfeder abhängig.

\subsection{MATERial}

Abbildung 6.8 zeigt den Einfluss des untersuchten Materials auf die Reibungsminderung. Dazu ist wieder die Reibungsdifferenz $\Delta F$ in Abhängigkeit von der Blattfederamplitude $A_{\mathrm{C}}$ für zehn verschiedene Normalkräfte von etwa 10 bis $200 \mathrm{nN}$ dargestellt, hier allerdings für amorphes (rot) und kristallines (blau) $\mathrm{Pd}_{77,5} \mathrm{Cu}_{6} \mathrm{Si}_{16,5}$ (PdCuSi). Auch hier ist ein quadratisches Verhalten erkennbar. Allerdings unterscheiden sich die Krümmungen deutlich.

Um die Differenz der Reibung $\Delta F$ für verschiedene Temperaturen zu messen, ist der Schallwandler des AFAM-Modus gegen eine selbstgebaute Heizstation getauscht worden. Sie besteht aus einer Aluminiumplatte, einem Heizdraht und einem Platin-Messwiderstand (Pt1000). Das kommerzielle Model 332 der Firma LakeShore misst den Widerstand des Pt1000 und reguliert mit dieser Information selbständig die Heizleistung und Heizrate und stellt so die Temperatur auf einen gewünschten Wert. Nachdem die Heizstation die gewünschte Temperatur erreicht hat, wird et- 
Abbildung 6.8 - Die Differenz der Reibung $\Delta F$ in $A b$ hängigkeit von der Blattfederamplitude $A_{C}$ für amorphes (rot) und kristallines (blau) $\mathrm{PdCuSi}$ für zehn verschiedene Normalkräfte. Die Kurven zeigen quadratisches Verhalten mit unterschiedlichen Krümmungen. Nach [Pfahll8].

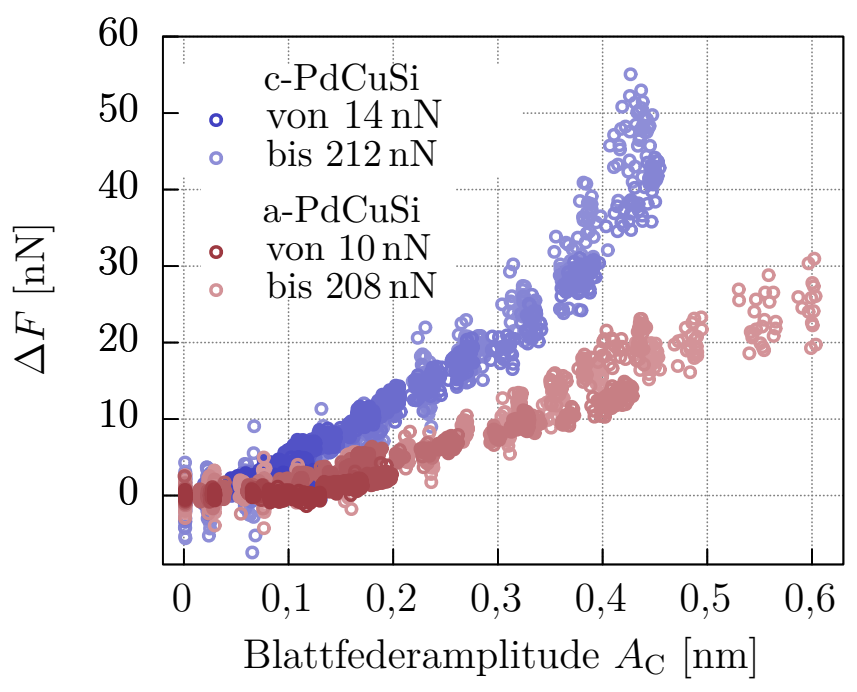

wa 30 Minuten gewartet, bevor die Messung gestartet wird, um sicherzugehen, dass auch die Probe die gewünschte Temperatur erreicht hat. Beachtet man diese Wartezeit nicht, führen thermische Ausdehnungen zu instabilen Messungen. Daher werden vor den Reibungsmessungen zusätzlich mehrere Kraft-Abstands-Kurven aufgenommen. Sollte sich die Probe noch im Aufheizprozess befinden, verschiebt sich die Kraft-Abstands-Kurve sichtbar in $z$-Richtung, da sich die Probe ausdehnt und die Oberfläche daher der Spitze näher kommt.

Abbildung 6.9 zeigt die Differenz der Reibung $\Delta F$ in Abhängigkeit von der Blattfederamplitude $A_{\mathrm{C}}$ von LSMO für verschiedene Temperaturen von Raumtemperatur in $5 \mathrm{~K}$-Schritten bis $365 \mathrm{~K}$. Kleine Blattfederamplituden bzw. niedrige Temperaturen zeigen das typische quadratische Verhalten. Bei höheren Temperaturen und größeren Amplituden hingegen kann man eine Sättigung von $\Delta F$ beobachten. Der Sättigungswert nimmt tendenziell mit zunehmender Temperatur ab.

\subsection{AnALYTiK}

Die Differenz der Reibung $\Delta F$ zeigt eine universelle Abhängigkeit von der Blattfederamplitude $A_{\mathrm{C}}$. Wie erwähnt wird im Prandtl-Tomlinson-Modell das nicht-lineare Kontaktpotential vernachlässigt. Das Anregen der Blattfeder führt aber dazu, dass sich die Blattfeder periodisch in diesem Potential bewegt, so dass auch nicht-lineare Effekte betrachtet werden müssen. In Kapitel 3.2.1 ist qualitativ beschrieben worden, wie eine Kraft, die abstandsabhängig anziehend oder abstoßend wirkt, auf die Blattfeder auswirkt. Zur Veranschaulichung wurde ein Lennard-Jones-Potential 


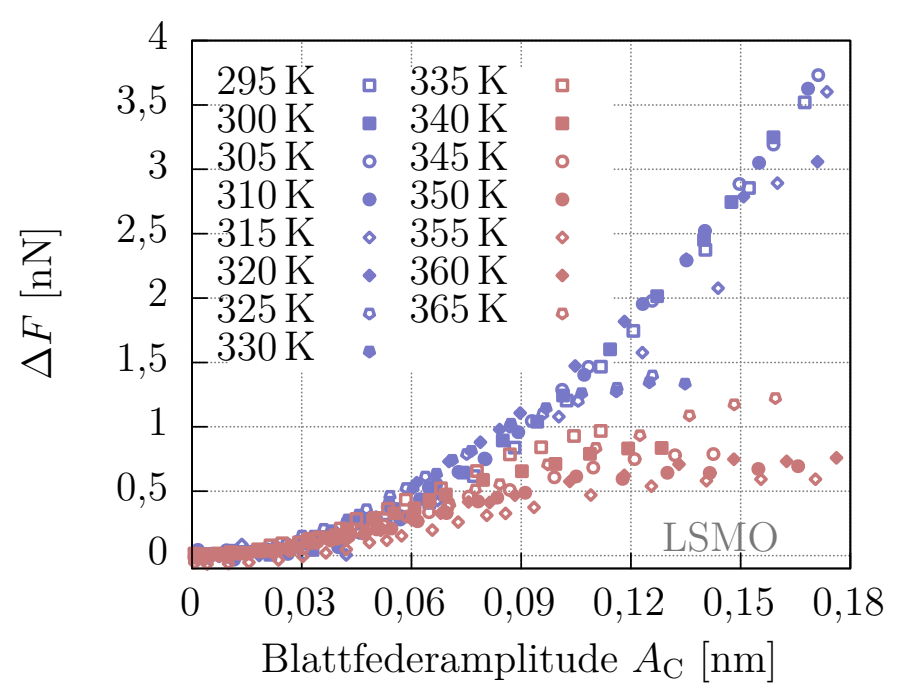

Abbildung 6.9 - Die Differenz der Reibung $\Delta F$ in Abhängigkeit von der Blattfederamplitude $A_{C}$ der LSMO in Probe für verschiedene Temperaturen. Nach [Pfahl18].

verwendet. Dieses Potential beschreibt jedoch die Wechselwirkungen zwischen zwei ungeladenen Atomen und ist daher nur bedingt zur Beschreibung der Kräfte zwischen Messspitze und Probenoberfläche geeignet.

Burnham et al. [Burnham93] diskutieren klassische Kontaktmodelle wie das Derjaguin-Muller-Toporov-Modell (DMT) [Derjaguin75] oder das Johnson-KendallRoberts-Modell (JKR) [Johnson71] und zeigen, dass ein semi-empirischer Ausdruck in Form einer Superposition beider Modelle die beste Beschreibung für numerische und experimentelle AFM-Daten ist. Dabei gilt für die Normalkraft einer Blattfeder im Kontakt mit einer Probe

$$
F_{\mathrm{N}}=\underbrace{\frac{4}{3} E^{*} \sqrt{R(-z)^{3}}}_{F_{\mathrm{A}}} \underbrace{-\sqrt{2 \pi \gamma E^{*}(-R z)^{3 / 2}}}_{F_{\mathrm{B}}} .
$$

Die abstoßende Kraft $F_{\mathrm{A}}$ resultiert aus dem Hertz'schen Kontaktmodell [Johnson85] und ist die Kraft, die erforderlich ist, um eine Kugel mit Radius $R$ und effektivem elastischen Modul $E^{*}$ um den Betrag von $z$ in einen elastischen Halbraum einzudrücken. Der Parameter $z$ beschreibt dabei die Distanz zwischen Spitze und Probenoberfläche und ist im Kontakt negativ. Die Kraft $F_{\mathrm{B}}$ ist eine adhäsive Kraft mit der Adhäsionskonstante $\gamma$. Der effektive elastische Modul $E^{*}$ ist über die Beziehung

$$
\frac{1}{E^{*}}=\frac{1-\nu_{\mathrm{S}}^{2}}{E_{\mathrm{S}}}+\frac{1-\nu_{\mathrm{P}}^{2}}{E_{\mathrm{P}}}
$$

gegeben, wobei $\nu_{\mathrm{S}}$ und $E_{\mathrm{S}}$ die Poissonzahl und der elastische Modul der Spitze und $\nu_{\mathrm{P}}$ und $E_{\mathrm{P}}$ die Poissonzahl und der elastische Modul der Probe ist. 
Regt man nun das System Blattfeder-Probe über den Schallwandler unterhalb der Probe bzw. den des Tapping-Modus an, führt dies zu einer sinusförmigen Distanzmodulation. Für die Distanz gilt dann

$$
z(t)=z_{0}+z_{1} \sin \omega t
$$

wobei $z_{0}$ die Distanz der Ruhelage - und auch die Distanz $z$ ohne Anregung - und $z_{1}$ die Amplitude ist. Durch die Nicht-Linearität der Normalkraft bezüglich z (Gl. 6.2) führt eine Distanzmodulation zur Veränderung der mittleren Normalkraft. Um dies zu berechnen, muss über eine Periode integriert werden. Dazu ist zunächst eine Taylor-Entwicklung der Normalkraft notwendig, die eine dimensionslose Variable fordert. Verwendet man $x(t)=z(t) / c$ mit $c=1 \mathrm{~m}$, gilt für die Distanz

$$
x(t)=\frac{z_{0}+z_{1} \sin \omega t}{c} .
$$

Die Taylor-Reihe zweiter Ordnung der Gleichung 6.2 an der Stelle $x_{0}$ ergibt dann

$$
F_{\mathrm{N}}(x(t)) \approx a\left(3 x^{2}+6 x x_{0}-x_{0}^{2}\right)-b\left(-3 x^{2}+30 x x_{0}+5 x_{0}^{2}\right)
$$

mit

$$
a=\frac{E^{*}}{6 x_{0}^{2}} \sqrt{-R\left(c^{3} x_{0}^{3}\right)} \quad \text { und } \quad b=\frac{1}{32 x_{0}^{2}} \sqrt{2 \pi \gamma E^{*}\left(-R c x_{0}\right)^{3 / 2}}
$$

Dadurch kann die Zeitintegration durchgeführt werden und es ergibt sich die mittlere Normalkraft unter Anregung

$$
\begin{aligned}
\Delta F_{\mathrm{N}, \text { on }} & =\frac{1}{2 \pi} \int_{0}^{2 \pi} F_{\mathrm{N}}(x(t)) \mathrm{d} t \\
& =F_{\mathrm{N}}(x(0))+\frac{3}{2} x_{1}^{2}(a+b) \\
& =F_{\mathrm{N}}(x(0))+\frac{z_{1}^{2}}{z_{0}^{2}}\left(\frac{E^{*}}{4} \sqrt{-R z_{0}^{3}}+\frac{3}{64} \sqrt{2 \pi \gamma E^{*}\left(-R z_{0}\right)^{\frac{3}{2}}}\right) .
\end{aligned}
$$

Da $x(0)=x_{0}$, entspricht die Kraft $F_{\mathrm{N}}(x(0))$ der Normalkraft $F_{\mathrm{N} \text {,off }}$ ohne Anregung aus Gleichung 6.2. Das bedeutet zunächst, dass die Normalkraft $\Delta F_{\mathrm{N} \text {,on }}$ unter Anregung größer ist als die Kraft $F_{\mathrm{N}, \text { off }}$ ohne. Außerdem fällt auf, dass ein Vorzeichenwechsel stattgefunden hat und nun auch der Term, der proportional zur adhäsiven Kraft $F_{\mathrm{B}}$ ist - also $b$ bzw. $\gamma$ enthält -, die Normalkraft erhöht. 

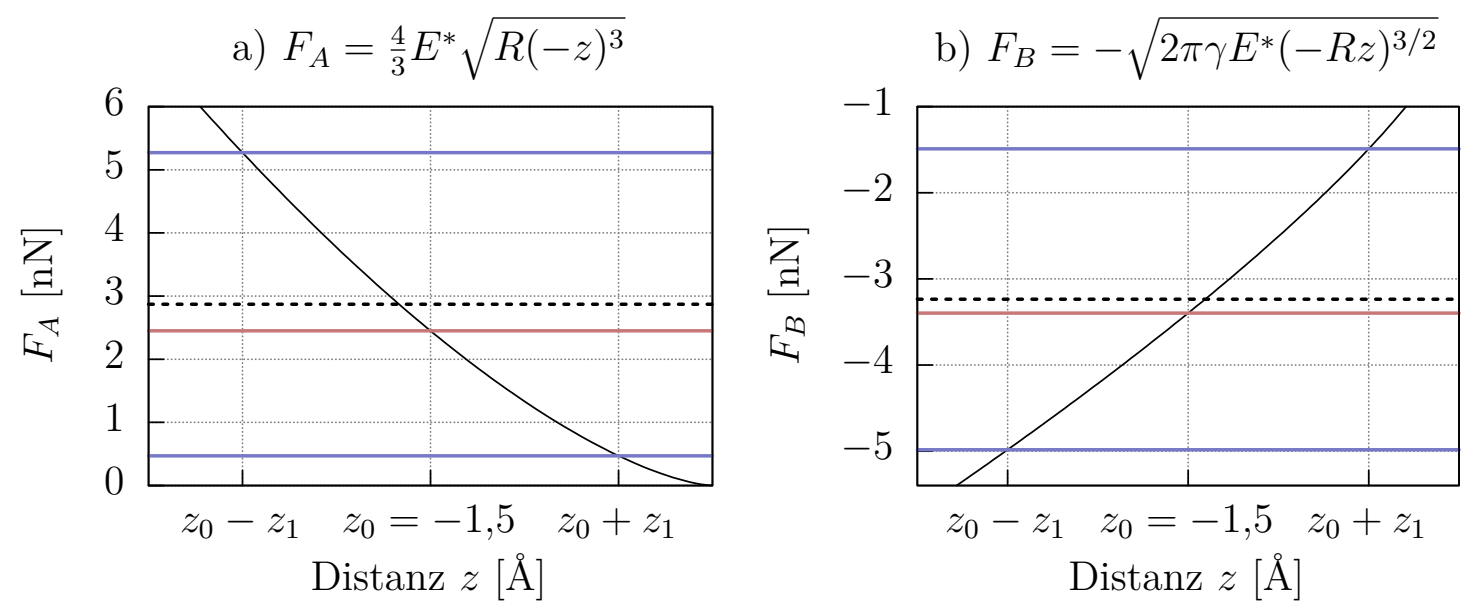

Abbildung 6.10 - Die Normalkraft aus Gl. 6.2 separiert in $F_{A}$ a) und $F_{B}$ b). In beiden Kräften führt eine Distanzmodulation mit Amplitude $z_{1}=0,5 \AA$ um $z_{0}=-1,5 \AA \mathrm{zu}$ einer Kraftmodulation zwischen den blauen Linien. Der zeitliche Mittelwert (gestrichelte Linie) verschiebt in beiden Fällen zu höheren Kräften als die Ruhelage (rote Linie). Die Anregung führt in beiden Fällen zu größerer Normalkraft. Es wurden die Werte $R=10 \mathrm{~nm}, E^{*}=10 \mathrm{GPa}$ und $\gamma=0,1 \mathrm{~N} \mathrm{~m}^{-1}$ verwendet.

Exemplarisch kann dies bildlich veranschaulicht werden. Dazu zeigen die Abbildungen $6.10 \mathrm{a}$ ) und b) die Kräfte $F_{\mathrm{A}}$ und $F_{\mathrm{B}}$ (schwarze Kurven) der Gleichung 6.2 separat in Abhängigkeit von der Distanz z. Die Variablen sind der Einfachheit halber auf $R=10 \mathrm{~nm}, E^{*}=10 \mathrm{GPa}$ und $\gamma=0,1 \mathrm{~N} \mathrm{~m}^{-1}$ gesetzt worden. Aus der Ruhelage - die hier auf $z_{0}=-1,5 \AA$ gesetzt worden ist - resultiert jeweils eine Kraft, die durch die roten Linien verdeutlicht wird. Ändert man die Distanz nun sinusförmig mit der Amplitude $z_{1}=1 \AA$, variiert sie zwischen $z_{0}-z_{1}=0,5 \AA$ und $z_{0}+z_{1}=2,5 \AA$. Dadurch variiert auch die Kraft zwischen einer maximalen und minimalen Kraft. Beide Extrema sind hier jeweils durch blaue Linien dargestellt. Die gestrichelte Linie zeigt den zeitlichen Mittelwert der Kraft unter Anregung. Man kann erkennen, dass sich der Wert im Vergleich zur Ruhelage bei beiden Kräften in Richtung größerer Kräfte verschiebt. Das bedeutet, dass die Nicht-Linearitäten der beiden Kräfte $F_{\mathrm{A}}$ und $F_{\mathrm{B}}$ die Normalkraft unter Anregung vergrößern und es gilt: Je größer die Amplitude $z_{1}$ ist, desto größer ist auch die Normalkraft. Arbeiten wie [Kolosov93, Hirsekorn97, Dinelli00] zeigen, dass die Blattfeder mit steigender Amplitude von der Probenoberfläche weggedrückt wird. In diesen Arbeiten wurden jedoch andere Parameter $\left(z_{0}\right.$ und $\left.z_{1}\right)$ gewählt. Eine detaillierte Beschreibung dazu kann in [Rabe02] gefunden werden.

Eine Anregung über einen Schallwandler führt also zu einer Erhöhung der Nor- 
Abbildung 6.11 - Die nichtlineare Funktion $F=a x^{2}$ zeigt beispielhaft, dass unter Anregung mit Amplitude $z_{1}$ um $z_{0}$ das arithmetische Mittel $0,5\left(F_{1}+F_{2}\right)$ größer als der zeitliche Mittelwert $F_{N, o n}$ und als die Ruhekraft $F_{N, \text { off ist. [Pfahl18]. }}$

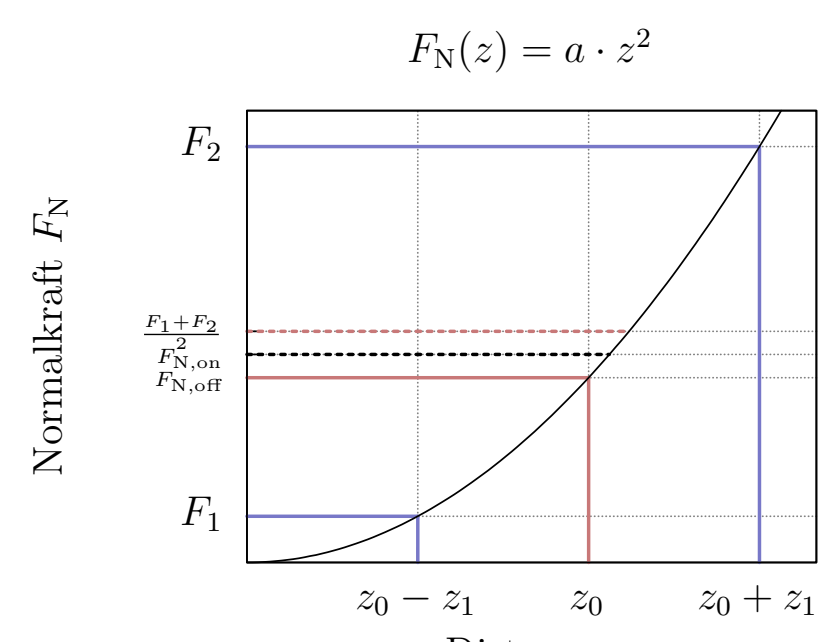

Distanz $z$

malkraft. Dieses steht allerdings im klaren Widerspruch zu den präsentierten Daten, da die Reibungskraft nicht abnehmen kann, wenn man die Auflagekraft vergrößert. Daher muss nun der Einfluss der Rückkopplung untersucht werden. [Pfahl18]

Während einer Messung mit dem AFM reagiert die Rückkopplung auf Höhenunterschiede der Probenoberfläche, indem es die Distanz anpasst. Dadurch dass die Anregung die Normalkraft verändert, greift auch hier die Rückkopplung ein. Man würde zunächst vermuten, dass sie - wie normalerweise gewünscht - dies so tut, dass die Auflagekraft und damit die Distanz konstant bleibt. Dies würde allerdings dazu führen, dass gar keine Reibungsänderung unter Anregung beobachtet werden kann. Die Rückkopplung des AFM setzt die Normalkraft auf den Mittelwert der maximalen und minimalen Kraft einer Periode. Abbildung 6.11 macht dies an der Funktion $F=a x^{2}$ deutlich. [Pfahl18] Auch hier ist die maximale Kraft $F_{2}$ bzw. die minimale Kraft $F_{1}$ unter Anregung mit blauen Linien gekennzeichnet. Die Normalkraft $F_{\mathrm{N} \text {,off }}$ ohne Anregung ist wieder als rote Linie dargestellt. Die zeitlich gemittelte Kraft $F_{\mathrm{N} \text {,on }}$ wird durch die schwarze gestrichelte Linie beschrieben. Dazu kommt nun die gemittelte Kraft $F_{\mathrm{AFM}}=\frac{F_{1}+F_{2}}{2}$ des AFM. Sie ist durch die rote gestrichelte Linie dargestellt und liegt höher als $F_{\mathrm{N} \text {,off }}$, aber auch höher als $F_{\mathrm{N} \text {,on }}$. Es sieht so aus, als ob $F_{\mathrm{N}, \text { on }}-F_{\mathrm{N}, \text { off }}=\frac{F_{1}+F_{2}}{2}-F_{\mathrm{N} \text {,on }}$ gilt, dass also die schwarze Linie mittig zwischen den roten Lininen liegt. Und tatsächlich ergibt sich ebendies auch mathematisch. Im Fall von Gleichung 6.2 gilt [Pfahl18]

$$
\frac{F_{1}+F_{2}}{2}=F_{\mathrm{N}, \mathrm{off}}+\underbrace{3 x_{1}^{2}(a+b)}_{F_{\mathrm{AFM}}} .
$$

Das AFM misst unter Anregung also eine zusätzliche Kraft $F_{\mathrm{AFM}}$ und die Rück- 
kopplung reduziert die Normalkraft um ebendiesen Wert, so dass

$$
\Delta F_{\mathrm{N}, \mathrm{AFM}}=F_{\mathrm{N}, \mathrm{off}}-F_{\mathrm{AFM}}
$$

gilt. Das AFM reduziert die Normalkraft also zu stark, wodurch die tatsächliche Normalkraft eine Funktion der anregenden Amplitude wird. Für die Differenz zwischen der Normalkraft ohne Anregung und der mit Anregung ergibt sich schließlich mit Gleichungen 6.8 und 6.6

$$
\begin{aligned}
\Delta F_{\mathrm{N}} & =F_{\mathrm{N}, \mathrm{off}}-\left(\Delta F_{\mathrm{N}, \mathrm{on}}-\Delta F_{\mathrm{N}, \mathrm{AFM}}\right) \\
& =\frac{z_{1}^{2}}{z_{0}^{2}}\left(\frac{E^{*}}{4} \sqrt{-R z_{0}^{3}}+\frac{3}{64} \sqrt{2 \pi \gamma E^{*}\left(-R z_{0}\right)^{\frac{3}{2}}}\right) .
\end{aligned}
$$

Mit dem ersten Amtonons'schen Gesetz (s. S. 9) ergibt sich schließlich die Beziehung

$$
\Delta F=\mu \frac{z_{1}^{2}}{z_{0}^{2}}\left(\frac{E^{*}}{4} \sqrt{-R z_{0}^{3}}+\frac{3}{64} \sqrt{2 \pi \gamma E^{*}\left(-R z_{0}\right)^{\frac{3}{2}}}\right)
$$

mit dem Reibungskoeffizienten $\mu$, der Amplitude $z_{1}$, der Ruhelage $z_{0}$, dem effektiven Modul $E^{*}$, dem Spitzenradius $R$ und der Adhäsionskonstante $\gamma$. Die Reibungsdifferenz $\Delta F$ ist also eine quadratische Funktion der Amplitude $z_{1}$ mit dem Reibungskoeffizienten $\mu$ als Vorfaktor. [Pfahl18]

\subsection{Messdaten und AnAlytische Beschreibung}

Da die gemessene Amplitude $A_{\mathrm{C}}$ proportional zur Amplitude $z_{1}$ ist (vgl. Abb. 6.4), ist die Differenz der gemessenen Kräfte $\Delta F=\Delta F_{\text {off }}-\Delta F_{\text {on }}$. Sie ist also eine quadratische Funktion der Amplitude $A_{\mathrm{C}}$, wobei die Krümmung proportional zum Reibungskoeffizienten $\mu$ ist, so dass

$$
\sqrt{\Delta F} \propto A_{\mathrm{C}} \sqrt{\mu}
$$

gilt. Die Abhängigkeit kann zunächst qualitativ anhand der Resonanzkurven überprüft werden. Abbildung 6.12 zeigt, dass die gemessenen Werte $\sqrt{\Delta F}$ mit der gemessenen Amplitude $A_{\mathrm{C}}$ sowohl für LSMO als auch für Silizium skalieren.

Außerdem können ermittelte Steigungen ins Verhältnis gesetzt werden, so dass man mit bekannten Reibungskoeffizienten $\mu_{0}$ andere Reibungskoeffizienten bestimmen kann. Es ist damit auch möglich, die Änderung der Reibungskonstante zu untersuchen. Abbildung 6.13 zeigt den Reibungskoeffizienten $\mu$ der LSMO-Probe in 


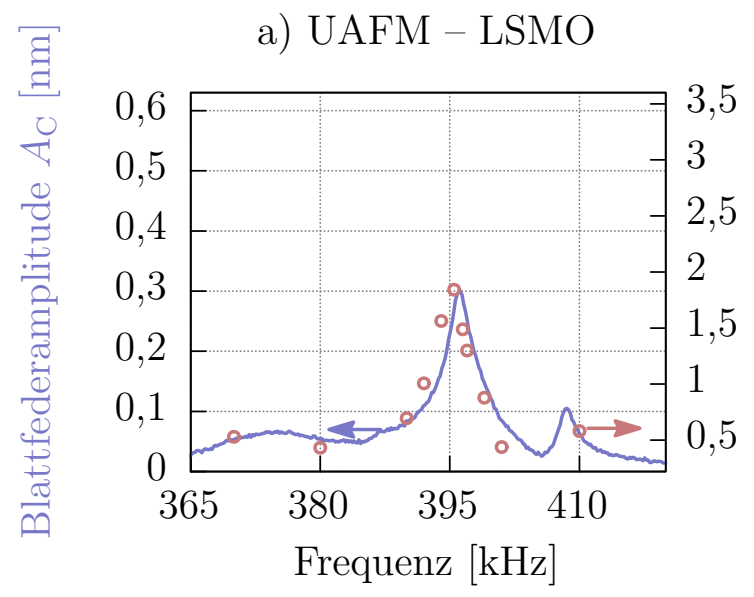

b) UAFM - Silizum
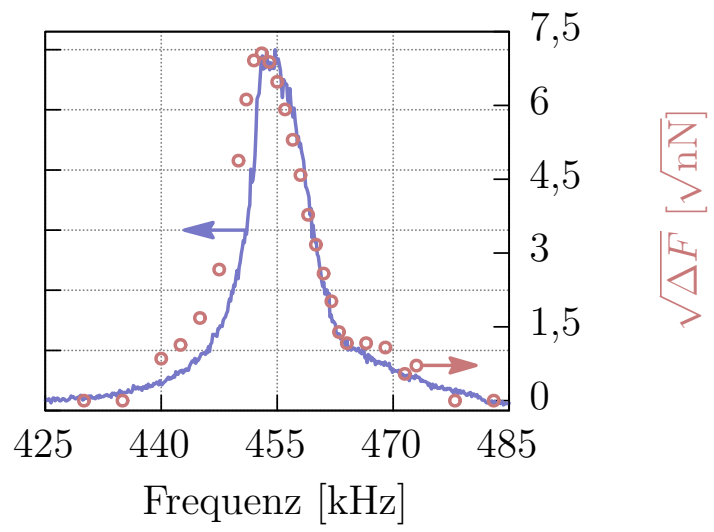

Abbildung 6.12 - Die Reibungskraftdifferenz $\Delta F$ skaliert mit der Blattfederamplitude $A_{C}$ gemäß $\sqrt{\Delta F} \propto A_{C} \sqrt{\mu}$. Die gemessenen Resonanzkurven und Reibungskraftdifferenzen $\Delta F$ auf LSMO a) und Silizium b) bestätigen dies.

Abhängigkeit von der Temperatur normiert auf die Steigung $\mu_{0}$ bei Raumtemperatur. Die gestrichelte Linie ist dabei eine Bezierkurve, die die Abnahme verdeutlicht. Im Temperaturbereich von $320 \mathrm{~K}$ bis $350 \mathrm{~K}$ fällt der Reibungskoeffizient auf etwa $40 \%$ des Raumtemperaturwertes. In der eingefügten Abbildung sind beispielhaft lineare Anpassungen der Daten bei $300 \mathrm{~K}$ und $360 \mathrm{~K}$ dargestellt.

Um die analytische Beschreibung 6.10 auch quantitativ zu verifizieren, müssen zunächst Reibunskoeffizient $\mu$, elastischer Modul $E^{*}$, Spitzenradius $R$ und Adhäsionskonstante $\gamma$ gemessen bzw. abgeschätzt werden.

\section{Abbildung $\quad 6.13 \quad-\quad \ddot{U} b e r$}

$\sqrt{\Delta F} \propto A_{C} \sqrt{\mu}$ kann durch lineare Fits der Reibungskoeffizient $\mu$ im Verhältnis zu einem anderen $\mu_{0}$ bestimmt werden. Der Reibungskoeffizient der LSMO-Probe nimmt im Vergleich zum Raumtemperaturwert $\mu_{0}$ mit der Temperatur ab. Nach [Pfahl18].

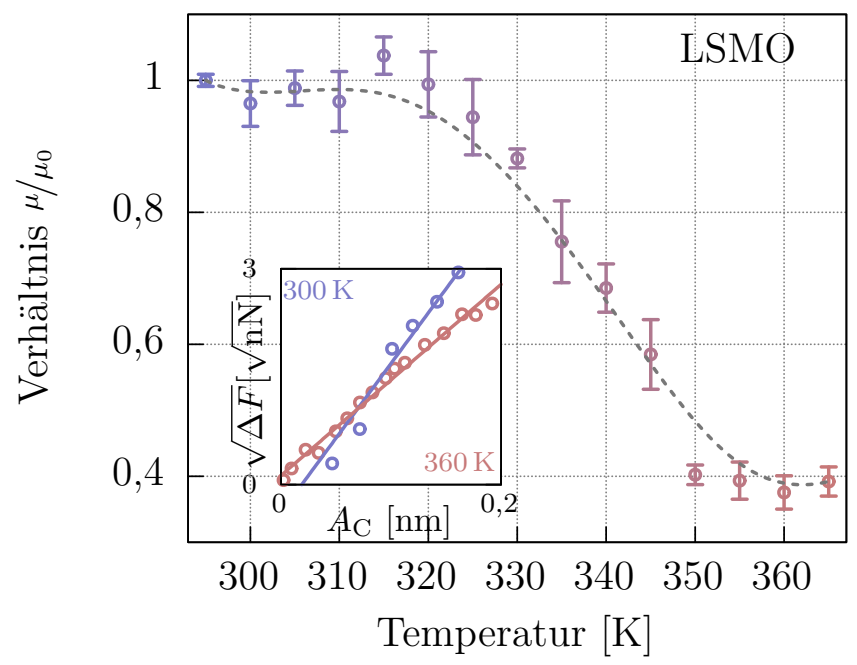




\subsubsection{Der effektive elastische Modul $E^{*}$}

Der effektive elastische Modul der Probe bzw. die benötigten Daten werden der Literatur entnommen. Die Spitze besteht aus Silizium, das einen Young-Modul von $G=169 \mathrm{GPa}$ und einen E-Modul von $E=50 \mathrm{GPa}$ hat. Zusammen mit dem Young-Modul $G=19,24 \mathrm{GPa}$ und E-Modul $E=50,07 \mathrm{GPa}$ [Dang-Hyok14] von LSMO berechnet sich der effektive Modul $E_{\mathrm{LSMO}}^{*}=41,2 \mathrm{GPa}$ [Pfahl17] und $E_{\mathrm{SI}}^{*}=$ $161,3 \mathrm{GPa}$. Die Werte für PdCuSi sind $E_{\mathrm{a}-\mathrm{PdCuSi}}^{*}=64 \mathrm{GPa}$ und $E_{\mathrm{c}-\mathrm{PdCuSi}}^{*}=71 \mathrm{GPa}$ [Wagner11b, Wagner11a].

\subsubsection{ReibungskoefFizient $\mu$}

Die Reibungskoeffizienten sind mit der klassischen FFM-Methode gemessen worden. Abbildung 6.14 zeigt die gemessene Reibungskraft der $\mathrm{Pd}_{77,5} \mathrm{Cu}_{6} \mathrm{Si}_{16,5}$-Proben und der $\mathrm{La}_{1-x} \mathrm{Sr}_{x} \mathrm{MnO}_{3}$-Probe in Abhängigkeit von der Normalkraft. Die Steigung einer linearen Anpassung an die Messdaten entspricht jeweils dem Reibungskoeffizienten $\mu$. Man findet hier $\mu_{\mathrm{c}-\mathrm{PdCuSi}}=0,35 \pm 0,02$ und $\mu_{\mathrm{LMSO}}=0,160 \pm 0,003$. Beim amorphen Material ist im Kräftebereich $40 \mathrm{nN}<F_{\mathrm{N}}<175 \mathrm{nN}$ eine lineare Anpassung mit kleinerem Fehler als eine Anpassung über den gesamten Wertebereich gefunden worden (gestrichelte Linie). Da die Messungen überwiegend in diesem Bereich durchgeführt worden sind, wird $\mu_{\mathrm{a}-\mathrm{PdCuSi}}=0,113 \pm 0,005$ verwendet.

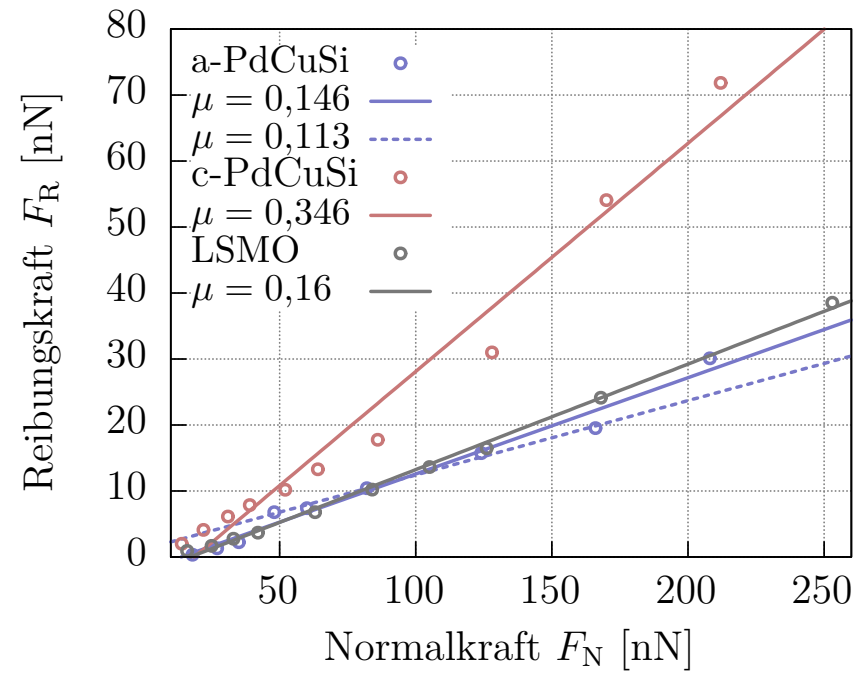

Abbildung $\quad 6.14 \quad-\quad A u s$ FFM-Messungen bestimmte Reibungskraft $\quad F_{R}$ der $\quad \mathrm{Pd}_{77,5} \mathrm{Cu}_{6} \mathrm{Si}_{16,5^{-}} \quad$ und $\mathrm{La}_{1-x} \mathrm{Sr}_{x} \mathrm{MnO}_{3}$-Proben. Die Linien stellen lineare Fits dar, deren Steigung dem jeweiligen Reibungskoeffizient entspricht. 


\subsubsection{SPITZENRADIUS $R$}

Der Spitzenradius wird anhand von Rasterelektronenmikroskop-Bildern auf $R=$ $60 \mathrm{~nm}$ geschätzt (s. Abb. 6.15). Man muss hier beachten, dass die Vermessung der Spitze nach sämtlichen Reibungsmessungen erfolgt ist. Eine neue Spitze hat typischerweise einen Spitzenradius von etwa $10 \mathrm{~nm}$. Um einen stabilen Zustand der Spitze zu generieren, wird diese vor den ersten Messungen einige Male auf der Probe bewegt, so dass bereits vor der ersten Messung ein größerer Radius erwartet werden kann. Da der exakte Spitzenradius während einer Messung nicht genau bestimmt werden kann, wird im Folgenden der obige Wert verwendet. Diese Annahme wird durch die gemessenen konstanten Kontaktresonanzfrequenzen gestützt, da eine Spitzenveränderung auch eine Frequenzveränderung zur Folge hätte.

Abbildung 6.15 - REM-Aufnahme der verwendeten Spitze. Der Radius beträgt etwa $60 \mathrm{~nm}$. Nach [Pfahl18].

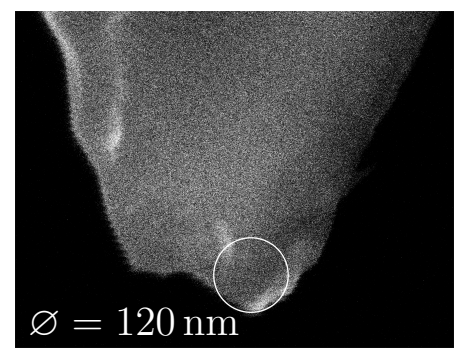

\subsubsection{ADHÄSIONSKONSTANTE $\gamma$}

Die Adhäsionskonstante bestimmt sich nach [Hirsekorn97] zu

$$
\gamma=\frac{k_{\mathrm{c}} D}{3 \pi R} \approx 0,063 \mathrm{~N} \mathrm{~m}^{-1}
$$

Dabei ist $D$ der Weg, den der $z$-Pizeo von der Probe wegfährt bis die Spitze sich von der Oberfläche löst, und kann daher aus der Kraft-Abstands-Kurve zu $D=$ $8,5 \mathrm{~nm}$ bestimmt werden. Die Federkonstante der Blattfeder ist gemessen worden und beträgt $k_{\mathrm{c}}=4,2 \mathrm{~N} \mathrm{~m}^{-1}$.

\subsubsection{Vergleich mit Messdaten}

Zur vollständigen analytischen Beschreibung fehlt nun nur noch die Kenntnis über die Amplitude $z_{1}$ und die Ruhedistanz $z_{0}$ zur Probe. Nimmt man zunächst an, dass die gemessene Amplitude $A_{\mathrm{C}}=z_{1}$, kann $z_{0}$ als Fit-Parameter genutzt werden. Dieser ist anhand der Messdaten der - willkürlich ausgesuchten - amorphen Probe 


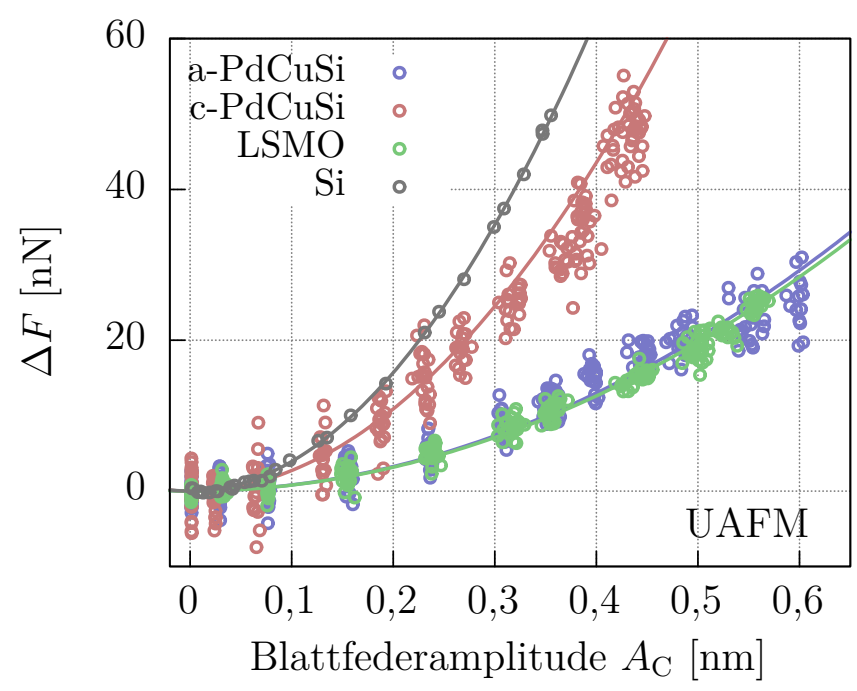

Abbildung 6.16 - Vergleich der analytischen Beschreibung mit den gemessenen Daten. Die Daten von a-PdCuSi sind zur Bestimmung von $z_{0}$ verwendet worden. Die analytische Darstellung beschreibt die Messdaten gut. Nach [Pfahl18].

bestimmt worden und beträgt $z_{0}=-0,055 \mathrm{~nm}$. Der Wert ist negativ, da sich die Spitze im Kontakt befindet [Hirsekorn97].

Abbildung 6.16 zeigt, dass die analytische Beschreibung mit den beschriebenen Parametern inklusive des Fit-Parameters $z_{0}$ auch für die Proben c-PdCuSi und LSMO eine gute Übereinstimmung mit den Messdaten zeigt. Daher kann diese Beschreibung verwendet werden, um unbekannte Reibungskoeffizienten zu bestimmen. Hier ist dies für Silizium durchgeführt worden. Der ermittelte Reibungskoeffizient ist $\mu_{\mathrm{Si}}=0,24$. Alsem et al. finden für polykristallines Silizium einen Reibungskoeffizienten von $\mu=0,24 \pm 0,07$ [Alsem08], Deng et al. untersuchten verschiedene Siliziumverbindungen und finden für ausgelagertes Siliziumdioxid auf Siliziumdioxid und auf reinem Silizium $\mu=0,20 \pm 0,02$ [Deng92]. Literaturwerte variieren allerdings stark. Vor allem die Luftfeuchtigkeit und die Messdauer haben einen Einfluss auf den Reibungskoeffizienten von Silizium. So finden Bhushan et al. [Bhushan97] über mehrere Messungen eine Varianz von $\mu=0,25 \pm 0,2$. Der gefundene Reibungskoeffizient stimmt dennoch mit Literaturwerten überein.

\subsection{NORMALKRAFTABHÄNGIGKEIT}

Bisher ist angenommen worden, dass die Normalkraft keinen direkten Einfluss auf die Differenz der Reibungskräfte $\Delta F$ hat. Dies soll nun genauer untersucht werden. Die Distanz zwischen Spitze und Probe $z_{0}=-0,055 \mathrm{~nm}$ ist durch eine quadratische Anpassung bestimmt worden. Sie kann für den Fall ohne externe Anregung aber auch über die Gleichung 6.2 in Abhängigkeit von der Normalkraft berechnet werden. 

a) Gleichung 6.13
b) Normalkraftabhängigkeit

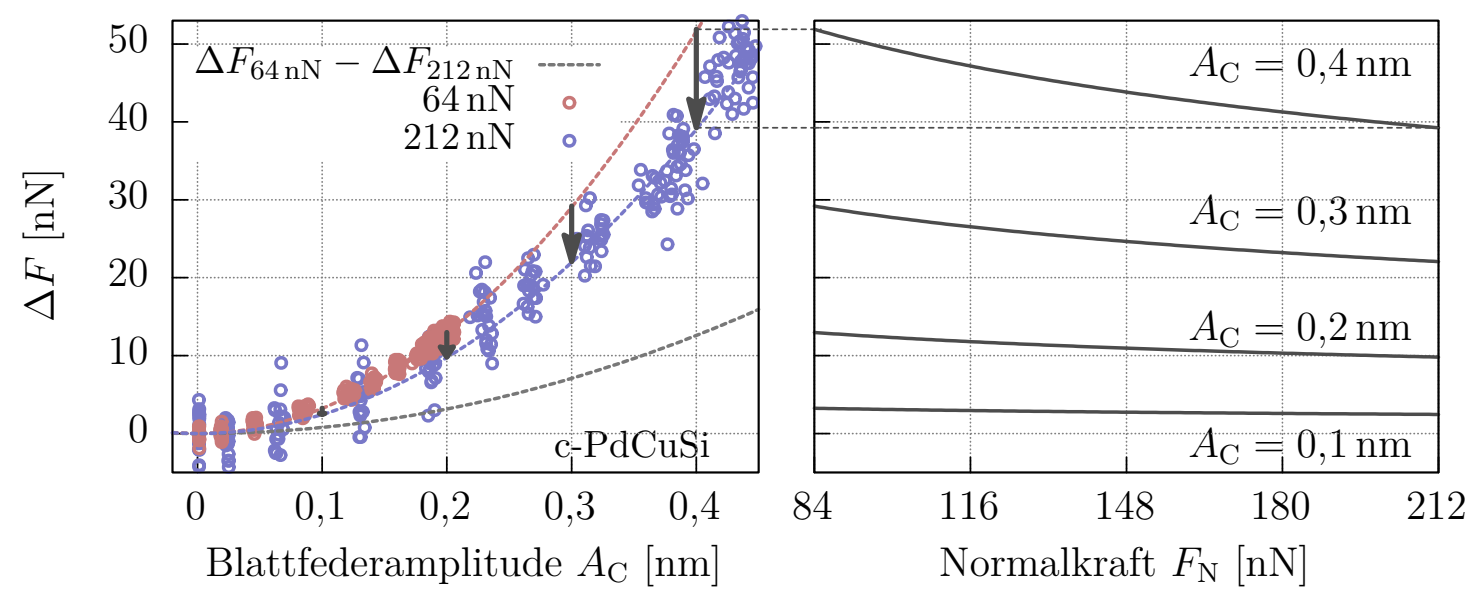

Abbildung 6.17 - Die Normalkraft führt zu minimalen Unterschieden der Differenz der Reibungskräfte $\Delta F$ in Abhängigkeit von der Blattfederamplitude $A_{C}$ der c-PdCuSiProbe - a) hier zu erkennen bei $F_{N}=212 \mathrm{nN}$ (blau) und $F_{N}=64 \mathrm{nN}$ (rot). b) Je größer die Amplitude ist, desto größer ist der Einfluss der Normalkraft.

Für c-PdCuSi ergibt sich beispielsweise

$$
z_{0}\left(F_{\mathrm{N}}=64 \mathrm{~nm}\right)=-0,29 \mathrm{~nm} \quad \text { und } \quad z_{0}\left(F_{\mathrm{N}}=212 \mathrm{~nm}\right)=-0,53 \mathrm{~nm} .
$$

Diese Werte liegen eine Größenordnung über dem gefitteten Wert. Dies kann erklärt werden, wenn man sich daran erinnert, dass die gemessene Blattfederamplitude $A_{\mathrm{C}}$ nur proportional zur Distanzamplitude $z_{1}$ ist. Für kleine Amplituden ist eine lineare Abhängigkeit

$$
z_{1}=k A_{\mathrm{C}}
$$

gezeigt worden und der Faktor $k$ kann durch einen Fit bestimmt werden. Anhand der Messdaten ergibt sich $k \approx 1,88$, was dem Wert der Lösung der ersten freien Mode $k_{1 \text {,free }}=1,875$ der Gleichung 3.12 auf Seite 41 entspricht. Die Gleichungen 6.11 und 6.10 ergeben somit die Beziehung

$$
\Delta F=\mu\left(A_{\mathrm{C}} k_{1, \text { free }}\right)^{2}\left(\frac{E^{*}}{4} \sqrt{-R z_{0}^{-1}}+\frac{3}{64} \sqrt{2 \pi \gamma E^{*}(-R)^{\frac{3}{2}} z_{0}^{-\frac{5}{2}}}\right) .
$$

Abbildung 6.17 a) zeigt die Messwerte der Differenz der Reibungskräfte $\Delta F$ in Abhängigkeit von der Blattfederamplitude $A_{\mathrm{C}}$ für c-PdCuSi bei $F_{\mathrm{N}}=212 \mathrm{nN}$ (blau) 
und $F_{\mathrm{N}}=84 \mathrm{nN}$ (rot) sowie Gleichung 6.13 mit den entsprechenden Werten. Es ist zu erkennen, dass die Messwerte für die beiden Kräfte nicht exakt übereinanderliegen. Dies resultiert aus größerem $z_{0}$ für größere Normalkräfte. Die analytische Beschreibung durch Gleichung 6.13 (rote bzw. blaue gestrichelte Linie) stellt daher eine Verbesserung zur vorherigen dar, da sie auch diesen Effekt der Normalkraftabhängigkeit berücksichtigt. Es sei hier darauf hingewiesen, dass die analytischen Kurven in Abbildung 6.17 a) keinen gefitteten Parameter mehr enthalten und Gleichung 6.13 so eine vollständige analytische Gleichung darstellt.

Abbildung 6.17 b) zeigt die Änderung der Reibungsdifferenz $\Delta F$ in Abhängigkeit von der Normalkraft $F_{\mathrm{N}}$ für verschiedene Blattfederamplituden für die c-PdCuSiProbe. Für große Amplituden - z. B. $A_{\mathrm{C}}=0,4 \mathrm{~nm}$ in blau - wird auch der Einfluss der Normalkraft groß. Auch die graue gestrichelte Linie in Abbildung 6.17 a) verdeutlicht anhand der Differenz $\Delta F_{84 \mathrm{nN}}-\Delta F_{212 \mathrm{nN}}$ in Abhängigkeit von der Amplitude den größeren Einfluss für größere Amplituden. Wird der Reibungskoeffizient aus der Krümmung der Messkurve $\Delta F$ gegen $A_{\mathrm{C}}$ bestimmen, muss der Normalkrafteinfluss berücksichtigt werden.

\subsubsection{ABWEICHEN DER DATEN}

Abbildung 6.18 zeigt weitere Messdaten bei Normalkräften, die zwischen denen aus Abbildung 6.17 a) liegen. Es ist zu erkennen, dass diese Daten eine Abweichung zur analytischen Beschreibung zeigen. Besonders fällt auf, dass die kleinste Krümmung bei den Kräften $F_{\mathrm{N}}=128 \mathrm{nN}$ und $F_{\mathrm{N}}=170 \mathrm{nN}$ auftritt. Da nach dem vorherigen Abschnitt davon ausgegangen wird, dass die analytische Beschreibung korrekt ist, muss das Abweichen von der analytischen Funktion eine andere Ursache haben.

Geht man davon aus, dass alle verwendeten Parameter konstant sind - sich $\gamma$, $E^{*}$ und vor allem auch $R$ also nicht ändern - muss dieses Verhalten vom Reibungskoeffizienten $\mu$ selbst stammen. Da der Parameter $\Delta F$ aus den zwei gemessenen Werten $\Delta F_{\text {on }}$ und $\Delta F_{\text {off }}$ gewonnen wird, enthält er zwei gemessene Reibungskräfte bei zwei unterschiedlichen Normalkräften. Da prinzipiell zwei Punkte ausreichen, um einen linearen Fit durchzuführen, kann aus jedem Messwert $\Delta F$ der Reibungskoeffizient $\mu$ bestimmt werden. Dabei muss der Unterschied zwischen $\Delta F_{\text {on }}$ und $F_{\text {off }}$ ausreichend groß sein, was bei großen Amplituden erreicht wird. Zur Bestimmung des Reibungskoeffizienten wird Gleichung 6.13 nach $\mu$ umgestellt. Außerdem kann mit Gleichung 6.9 und Kenntnis der Amplitude $z_{1}=A_{\mathrm{C}} k_{1 \text {,free }}$ die Reduzierung der Normalkraft und damit auch die Normalkraft $F_{\mathrm{N}}=F_{\mathrm{N}, \text { off }}-\Delta F_{\mathrm{N}, \text { on }}\left(z_{1}\right)$ bestimmt werden. 
Abbildung 6.18 - Die Reibungskraftdifferenz $\Delta F$ in Abhängigkeit von $A_{C}$ der c-PdCsuSiProbe für fünf Normalkräfte. Im Bereich von $F_{N} \approx 100 \mathrm{nN}$ kommt es zur Abweichung von der analytischen Beschreibung.

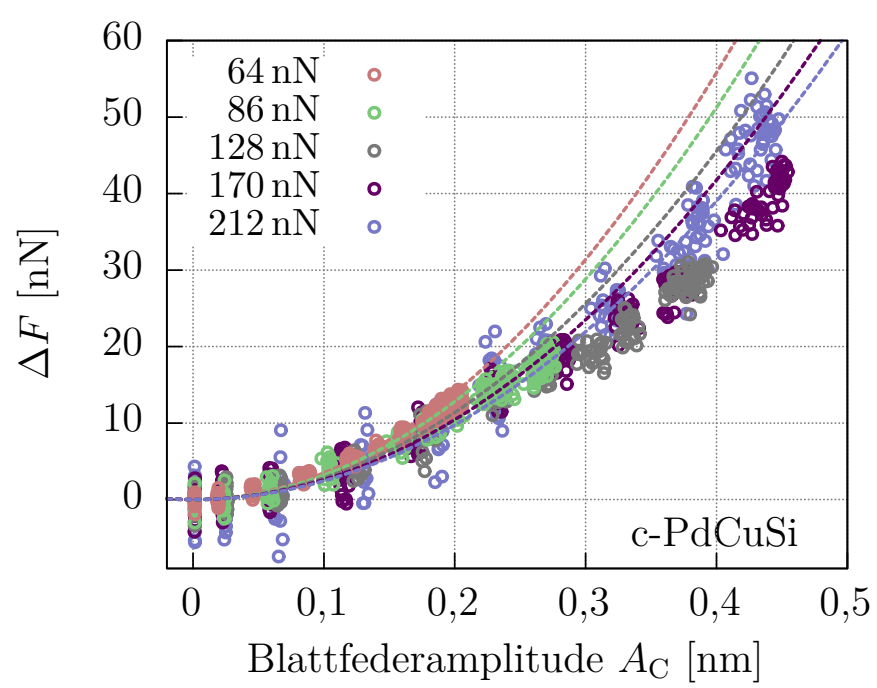

Somit ergibt sich, wie im Fall von c-PdCuSi in Abbildung 6.19 a) dargestellt, eine Abhängigkeit des Reibungskoeffizienten von der Normalkraft. Daten mit zu kleinen oder zu großen Amplituden sind hier entfernt worden, da kleine Amplituden zu großen Fehlern in $\mu$ und große Amplituden zum Abweichen der linearen Abhängigkeit der Amplituden führen. Für sehr kleine Normalkräfte steigt der Reibungskoeffizient linear an, bis er bei einem Maximalwert von $\mu \approx 0,5$ wieder abnimmt. Außerdem ist über den vermessen Kräftebereich ein Minimum zu erkennen, das durch die gestrichelte Linie angedeutet ist. Abbildung 6.19 b) zeigt einen genaueren Blick auf die gemessene Reibungskraft $F_{\mathrm{R}}$ in Abhängigkeit von der Normalkraft $F_{\mathrm{N}}$ (vgl. Abb. 6.14). Auch hier ist im vermessenen Kräftebereich eine Änderung der Steigung $\mu \mathrm{zu}$ erkennen. Es sind zwei weitere lineare Anpassungen für $F_{\mathrm{N}}<100 \mathrm{nN}$ (rot) mit $\mu=0,216 \pm 0,004$ und für $F_{\mathrm{N}}>100 \mathrm{nN}$ (grau) mit $\mu=0,486 \pm 0,037$ eingezeichnet. Der Schnittpunkt dieser Geraden liegt bei $F_{\mathrm{N}} \approx 110 \mathrm{nN}$. Die so ermittelten Reibungskoeffizienten sind in Abbildung 6.19 a) als horizontale Linien eingetragen.

Abbildung 6.20 zeigt die gleiche Auftragung für die amorphe Probe. Hier sind keine Minima oder Maxima zu erkennen. Der Reibungskoeffizient variiert in beiden Auftragungen ähnlich und im gleichen Wertebereich.

Auch der Reibungskoeffizient der LSMO-Probe zeigt keine Maxima oder Minima in Abhängigkeit von der Normalkraft (s. Abb. 6.21). Bei kleinen Normalkräften $F_{\mathrm{N}} \leq 40 \mathrm{nN}$ ist der Reibungskoeffizient $\mu$ in beiden Auftragungen leicht erniedrigt. 
a) $\mu$ gegen $F_{\mathrm{N}}$

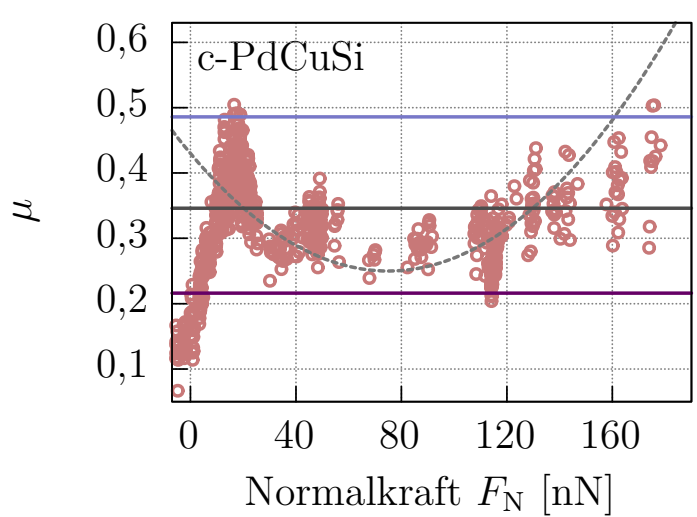

b) $\mu$ aus $F_{\mathrm{R}}$ gegen $F_{\mathrm{N}}$

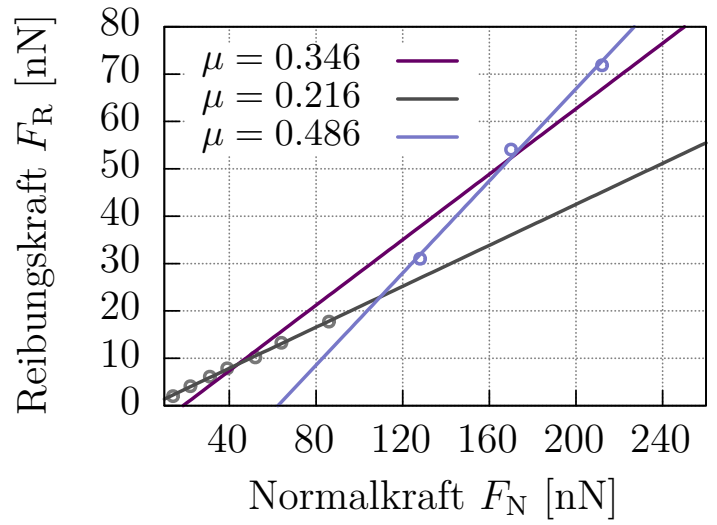

Abbildung 6.19 - Der Reibungskoeffizient $\mu$ kann aus jedem Wert $\Delta F$ bestimmt werden. a) Im Fall von c-PdCuSi ist ein Minimum bei $F_{N} \approx 75 \mathrm{nN}$ zu erkennen. b) Auch in FFM-Messungen kann in der Nähe dieser Kraft eine Änderung des Reibungskoeffizienten gemessen werden.

a) $\mu$ gegen $F_{\mathrm{N}}$

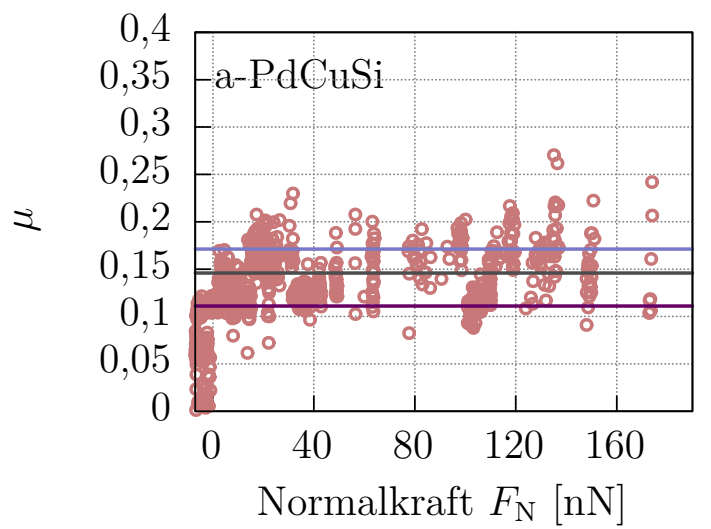

b) $\mu$ aus $F_{\mathrm{R}}$ gegen $F_{\mathrm{N}}$

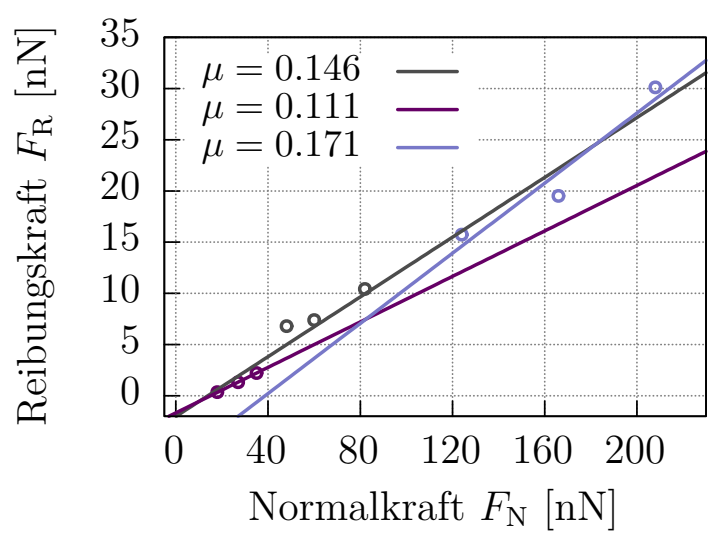

Abbildung 6.20 - Der Reibungskoeffizient $\mu$ kann aus jedem Wert $\Delta F$ bestimmt werden. a) Im Fall von a-PdCuSi zeigt der Reibungskoeffizient eine Streuung um den Wert, der auch in FFM-Messungen gefunden wird. b) In den FFM-Messungen können auch andere Steigungen gefunden werden. 
a) $\mu$ gegen $F_{N}$

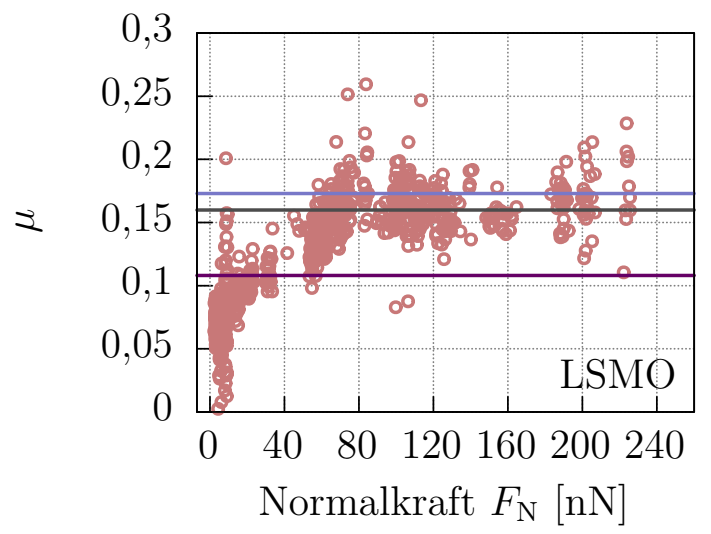

b) $\mu$ aus $F_{\mathrm{R}}$ gegen $F_{\mathrm{N}}$

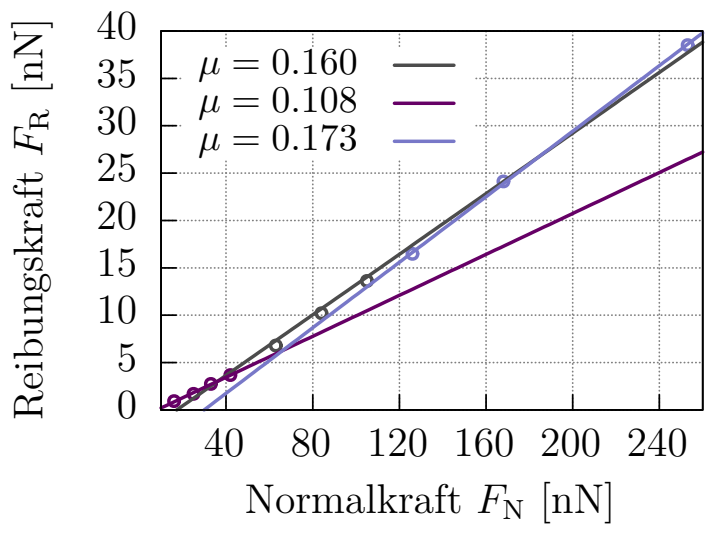

Abbildung 6.21 - Der Reibungskoeffizient $\mu$ der LSMO Probe streut um den Wert, der auch in FFM-Messungen gefunden wird. a) Unterhalb der Normalkraft $F_{N} \leq 40 \mathrm{~nm}$ ist der Reibungskoeffizient kleiner als bei größeren Normalkräften. b) Das findet man auch in FFM-Messungen. 


\subsection{Weitere AuftragungsmöGlichKeiten}

Es wurde gezeigt, dass der Wert $\Delta F$ aus analytischer Sicht hervorragend dazu geeignet ist, den Reibungskoeffizienten zu studieren. Natürlich gibt es auch weitere Abhängigkeiten. Zum Beispiel kann der prozentuale Abfall der Reibung untersucht werden, wenn man sich $\Delta F / \Delta F_{\text {off }}$ anschaut. Intuitiv mag man annehmen, dass der prozentuale Abfall der Reibung von der Normalkraft abhängen muss. Je größer diese ist, desto größere Amplituden sind notwendig, um die Reibung zu reduzieren. Abbildung 6.22 bestätigt am Beispiel der c-PdCuSi-Probe diese Annahme.

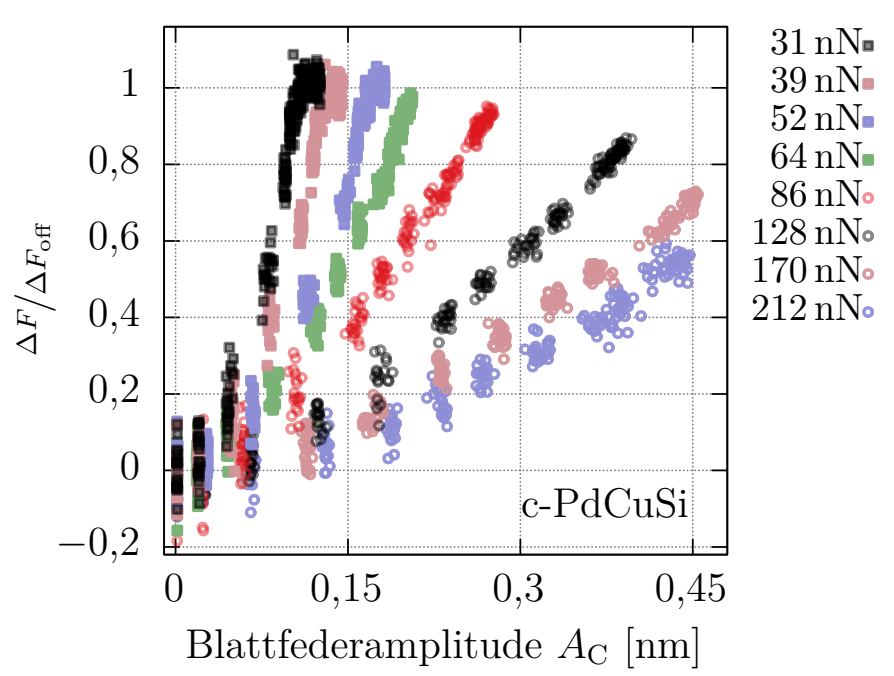

Abbildung 6.22 - Die prozentuale Reibungsminderung $\Delta F / \Delta F_{\text {off }}$ der c-PdCuSi Probe für verschiedene Normalkräfte. Je gröBer die Normalkraft ist, desto größere Amplituden sind notwendig, um die Reibung zu mindern.

Bei größter Auflagekraft von $F_{\mathrm{N}}=212 \mathrm{nN}$ ist die Reibung bei einer Amplitude von $A_{\mathrm{C}}=0,15 \mathrm{~nm}$ um etwa $10 \%$ reduziert, wohingegen bei gleicher Amplitude die Reibung bei einer Normalkraft von $F_{\mathrm{N}}=52 \mathrm{nN}$ bereits um $80 \%$ reduziert ist. Für kleinere Normalkräfte ist sie sogar vollständig unterdrückt.

Aus der analytischen Beschreibung kann der Einfluss der Normalkraft bestimmt werden. Mit den Gleichungen 6.1, 6.6 und 6.4 sowie dem 1. Amontons'schem Gesetz (s. S. 9) gilt

$$
\begin{aligned}
\frac{\Delta F}{\Delta F_{\mathrm{off}}} & =\frac{\Delta F_{\text {off }}-\Delta F_{\text {on }}}{\Delta F_{\text {off }}}=1-\frac{\Delta F_{\text {on }}}{\Delta F_{\text {off }}} \\
& =1-\frac{\mu \Delta F_{\mathrm{N}, \text { on }}}{\mu F_{\mathrm{N}, \text { off }}} \\
& =1-\frac{F_{\mathrm{N}, \text { off }}-\frac{3}{2} x_{1}^{2}(a+b)}{F_{\mathrm{N}, \text { off }}}=\frac{\frac{3}{2} x_{1}^{2}(a+b)}{F_{\mathrm{N}, \mathrm{off}}}
\end{aligned}
$$


Abbildung 6.23 - Der Einfluss der Normalkraft kann herausgerechnet werden, so dass die Messdaten eine Normalparabel beschreiben sollten. Bei kleineren Normalkräften kommt es zur Abweichung davon.

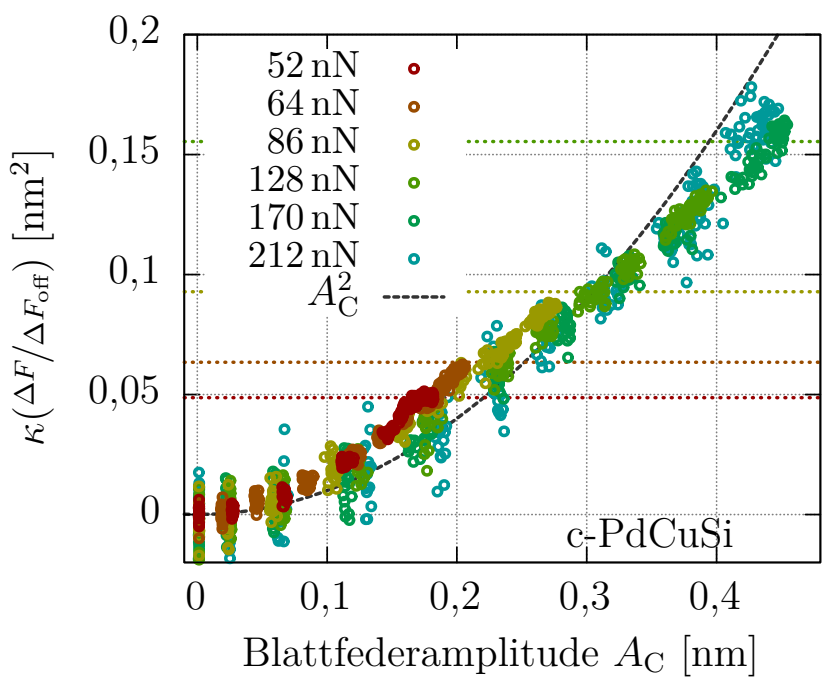

Hier ist $\kappa$ ein Parameter, der die Normalkraftabhängigkeit über die Ruhedistanz $z_{0}$ enthält, so dass er für eine feste Normalkraft konstant ist. Multipliziert man die prozentuale Reibungsminderung $\Delta F / \Delta F_{\text {off }}$ mit $\kappa$, müssen Messdaten gemäß Gleichung 6.16 - unabhängig von der Normalkraft - eine Normalparabel beschreiben. Abbildung 6.23 zeigt dies anhand der Daten der c-PdCuSi-Probe. Hier ist die gestrichelte schwarze Linie eine Normalparabel, die die Messwerte gut beschreibt. Die horizontalen gepunkteten Linien zeigen den Wert $\kappa$ für die Messdaten der jeweiligen Farbe. Für große Amplituden wird $\Delta F / \Delta F_{\text {off }}=1$, so dass die Messdaten für große Amplituden gegen $\kappa$ streben müssen. Abbildung 6.23 zeigt das beispielsweise für $F_{\mathrm{N}}=52 \mathrm{nN}$. Die Daten beschreiben zunächst bis etwa $A_{\mathrm{C}} \approx 0,1 \mathrm{~nm}$ die Normalparabel, weichen dann nach oben ab (größere Krümmung) und sättigen schließlich bei $A_{\mathrm{C}} \approx 0,16 \mathrm{~nm}$ mit dem Wert $\kappa$. Ein Abweichen von der Normalparabel bedeutet wieder, dass sich der Reibungskoeffizient in Abhängigkeit von der Normalkraft ändert. Beschreiben die Messdaten eine Krümmung $>1$, ist nach Gleichung 6.16 der Reibungskoeffizient erhöht, ist die Krümmung $<1$, ist er erniedrigt. 

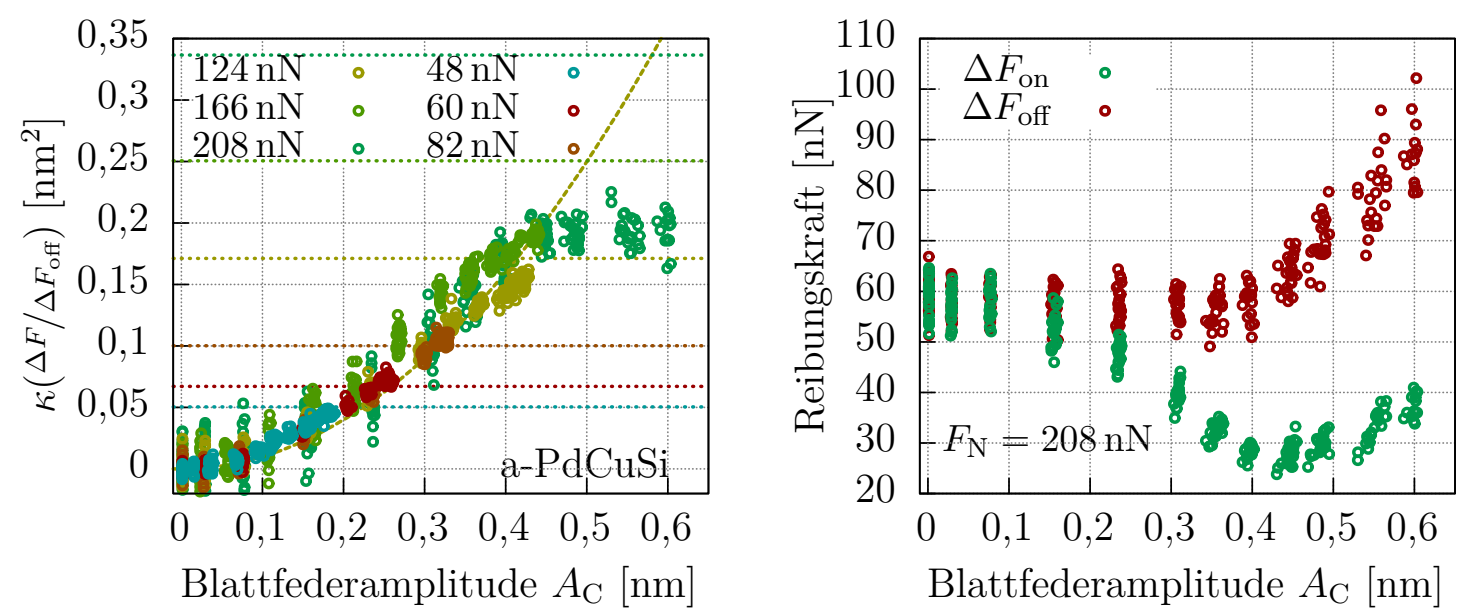

Abbildung 6.24 - a) Der Einfluss der Normalkraft kann herausgerechnet werden, so dass die Messdaten der amorphen Probe eine Normalparabel beschreiben sollten. Bei

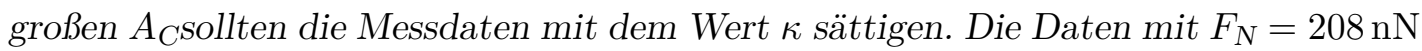
sättigen allerdings deutlich früher. b) Die Reibungskraftwerte $\Delta F_{\text {off }}$ und $\Delta F_{\text {on }}$ der aPdCuSi-Probe bei der Normalkraft $F_{N}=208 \mathrm{nN}$ steigen ab $A_{C} \approx 0,42 \mathrm{~nm}$ wieder an.

Abbildung 6.24 a) stellt die Messdaten der amorphen Probe in der gleichen Auftragung dar. Im vorherigen Abschnitt ist gezeigt worden, dass die amorphe Probe weniger Varianz im Reibungskoeffizienten zeigt. Die Messdaten beschreiben die Normalparabel besser als die des kristallinen Gegenstücks, was auch hier bedeutet, dass es weniger Varianz im Reibungskoeffizienten gibt. Jedoch scheint es, dass die Messdaten bei der Normalkraft $F_{\mathrm{N}}=208 \mathrm{nN}$ ab einer Amplitude von $A_{\mathrm{C}} \approx 0,42 \mathrm{~nm}$ sättigen, obwohl $\kappa$ noch nicht erreicht ist. Abbildung 6.24 b) zeigt die zugehörigen Reibungskraftwerte $\Delta F_{\text {off }}$ und $\Delta F_{\text {on }}$. Unterhalb $A_{\mathrm{C}} \approx 0,42 \mathrm{~nm}$ ist die Reibungskraft ohne Anregung konstant, und die mit Anregung beschreibt das erwartete quadratische Verhalten. Oberhalb $A_{\mathrm{C}} \approx 0,42 \mathrm{~nm}$ steigen beide Werte in Abhängigkeit von der Amplitude an. Da die Reibungskraft unter Anregung mit steigender Amplitude zunimmt und das sogar Auswirkungen auf die Reibungskraft ohne Anregung hat, muss sich etwas Grundlegendes ändern. Entweder ändert sich die Spitzenform oder aber etwas im Probensystem.

Dadurch dass das gleiche Verhalten - zwar weniger stark ausgeprägt - auch bei der vorhergehenden Messung mit $F_{\mathrm{N}}=166 \mathrm{nN}$ gefunden wird, kann ausgeschlossen werden, dass die Spitze die Ursache ist, da in der direkt anschließenden Messung an anderer Probenstelle die Reibungskraft ohne Anregung wieder kleiner und bis 


\begin{abstract}
Abbildung 6.25 - Für hohe Temperaturen zeigt die LSMOProbe Abweichungen von der Normalparabel. Mit steigender Amplitude $A_{C}$ wird das untersuchte Probenvolumen kleiner. Der Einfluss der Oberfläche wird dadurch größer und könnte daher die Ursache sein.
\end{abstract}

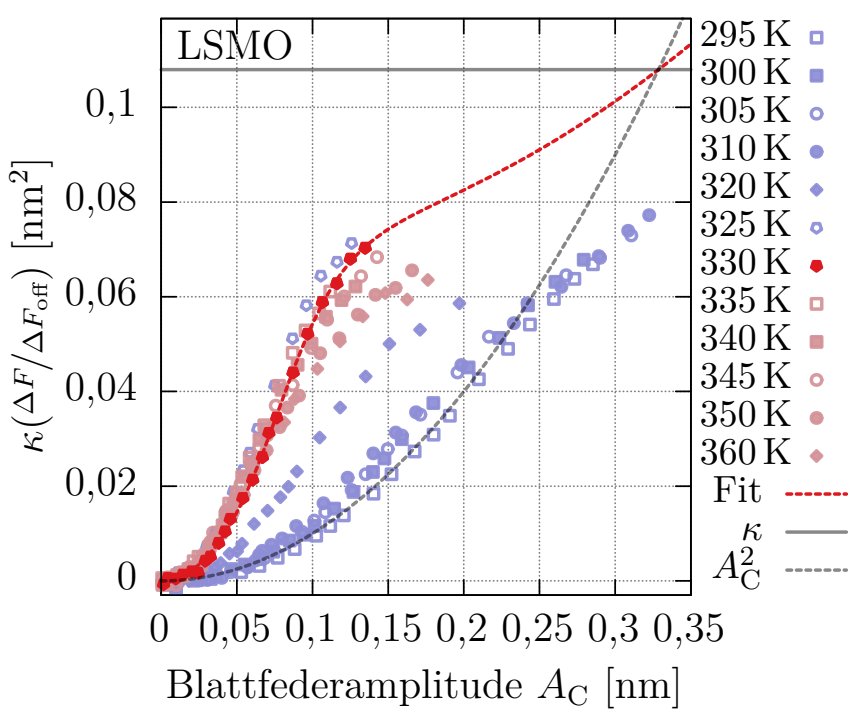

zur kritischen Amplitude $A_{\mathrm{C}} \approx 0,42 \mathrm{~nm}$ konstant ist.

Eine große Amplitude bedeutet zwar, dass die über die Zeit integrierte Normalkraft kleiner wird, durch die periodische Bewegung der Blattfeder wird die Kraft aber kurzzeitig größer sein als die eingestellte Normalkraft $F_{\mathrm{N}}=208 \mathrm{nN}$. Man könnte also vermuten, dass eine bestimmte Kraft erreicht ist, die etwas im Probensystem verändert. Dadurch dass auch die Reibungskraft ohne Anregung ab $A_{\mathrm{C}} \approx 0,42 \mathrm{~nm}$ ansteigt, muss die Änderung stabil sein. Ein ähnlicher Effekt ist in der kristallinen Probe nicht gemessen worden. In Kapitel 8 wird auf dieses Verhalten eingegangen.

Abbildung 6.25 zeigt dieselbe Auftragung für die Messdaten verschiedener Temperaturen der LSMO-Probe. Hier sind Abweichungen von der Normalparabel zu erkennen. Im Gegensatz zu den PdCuSi-Proben sind hier alle Messreihen bei gleicher Normalkraft durchgeführt worden, so dass $\kappa$ für alle Temperaturen gleich ist. Ähnlich wie im vorangegangenen Kapitel muss man also davon ausgehen, dass sich der Reibungskoeffizient abhängig von der Amplitude ändert.

Es ist zunächst anzunehmen, dass der materialspezifische Reibungskoeffizient der LSMO sich nicht grundlegend mit der Amplitude ändert. Was sich jedoch unter Anregung ändert, ist die Normalkraft und damit das untersuchte Probenvolumen. Wird hier von einer Deckschicht - egal ob chemisch, physikalisch oder thermisch - ausgegangen, die einen anderen Reibungskoeffizienten als das BulkMaterial aufweist, untersucht man mit steigender Amplitude einen größeren Anteil dieser Schicht. Wird davon ausgegangen, dass sich der Reibungskoeffizient unter steigender Anregung vom Bulk-Wert $\mu_{2}$ zu dem der Deckschicht $\mu_{1}$ ändert, kann 
dies mit einer der Fermi-Dirac-Verteilung analogen Verteilung gemäß

$$
\mu_{\mathrm{FD}}\left(A_{\mathrm{C}}\right)=\frac{\mu_{2}-\mu_{1}}{e^{b^{-1}\left(A_{\mathrm{C}}-A_{0}\right)}+1}+\mu_{1}
$$

beschreiben, wobei $A_{0}$ der Amplitudenwert ist, bei dem der Reibungskoeffizient dem Mittelwert 0.5 $\left(\mu_{1}+\mu_{2}\right)$ entspricht und $b$ die Breite der Änderung angibt. Nimmt man an, dass ohne Anregung der Einfluss der Deckschicht vernachlässigt werden kann, wird aus Gleichung 6.14

$$
\begin{aligned}
\frac{\Delta F}{F_{\text {off }}} & =1-\frac{\mu_{\mathrm{FD}}}{\mu_{2}} \frac{\Delta F_{\mathrm{N}, \text { on }}}{F_{\mathrm{N}, \text { off }}} \\
& =1-\frac{\mu_{\mathrm{FD}}}{\mu_{2}}\left(1-\kappa^{-1} A_{\mathrm{C}}^{2}\right) \\
\kappa \frac{\Delta F}{F_{\text {off }}} & =\kappa-\frac{\mu_{\mathrm{FD}}}{\mu_{2}}\left(\kappa-A_{\mathrm{C}}^{2}\right) .
\end{aligned}
$$

Da der Bulk-Wert $\mu_{2}$ bereits bestimmt ist (vgl. Abb. 6.13), wird er hier auf 1 gesetzt, so dass ein Fit möglich wird. Abbildung 6.25 zeigt mit der roten gestrichelten Linie einen Fit am Beispiel der roten Messdaten zur Temperatur $T=330 \mathrm{~K}$. Es resultieren dadurch die auf $\mu_{2}$ normierten Reibungskoeffizienten sowie die Breite $b$ und die Amplitude $A_{0}$. Aufgrund der quadratischen Form der Messkurven der Temperaturen 295, 300 und $315 \mathrm{~K}$ divergiert hier der Fit. Abbildung 6.26 a) zeigt das Verhältnis von $\mu_{1} \mathrm{zu} \mu_{2}$. Dies zeigt ein Minimum bei $T \approx 325 \mathrm{~K}$. Der Wert $\mu_{1}$ ist in b) dargestellt und zeigt ab der Temperatur $T \approx 325 \mathrm{~K}$ sättigendes Verhalten mit $\mu_{1} \approx 0,05$. Der beobachtete Abfall des Reibungskoeffizienten in diesem Temperaturbereich kommt also nicht von der Deckschicht, sondern vom Bulk-Material. Damit kann thermisches Hüpfen an der Oberfläche als Ursache ausgeschlossen werden.

Abbildung 6.27 zeigt die ermittelten Werte $A_{0}$ und $b$. Der Wert $A_{0}$ gibt den Wert der Blattfederamplitude $A_{\mathrm{C}}$ an, bei dem der Reibungskoeffizient den Mittelwert von Bulk- und Deckschichtwert annimmt. Je größer die Blattfederamplitude $A_{\mathrm{C}}$ ist, desto kleiner ist die zeitlich gemittelte Normalkraft $\Delta F_{\mathrm{N}, \mathrm{on}}$. Eine Verringerung der Normalkraft $\Delta F_{\mathrm{N} \text {,on }}$ führt wiederum zur Reduzierung des Kontaktradius $a_{\mathrm{c}}$ der Kontaktfläche von Spitze und Probe. Das Spannungsfeld in der Probe unterhalb der Spitze ist inhomogen, so dass es schwierig ist eine Tiefenwirkung abzuschätzen. Jedoch erreicht das Spannungsfeld eine maximale Schubspannung $p_{\max }$ bei einer 
a) Verhältnis $\mu_{1} / \mu_{2}$

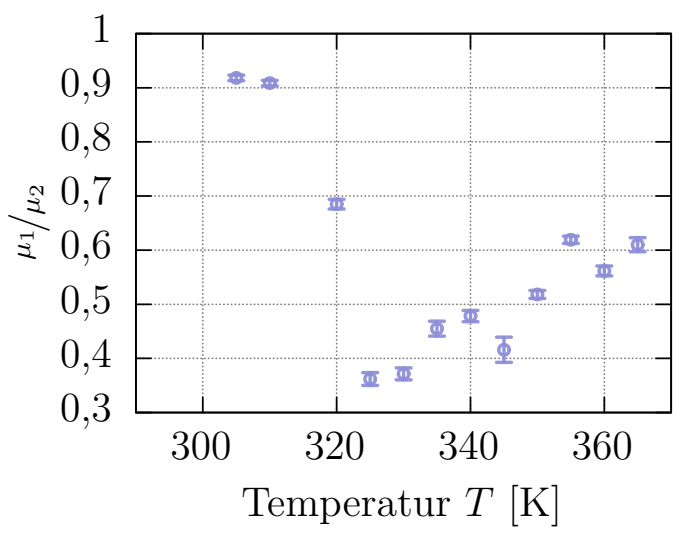

b) Reibungskoeffizient $\mu_{1}$

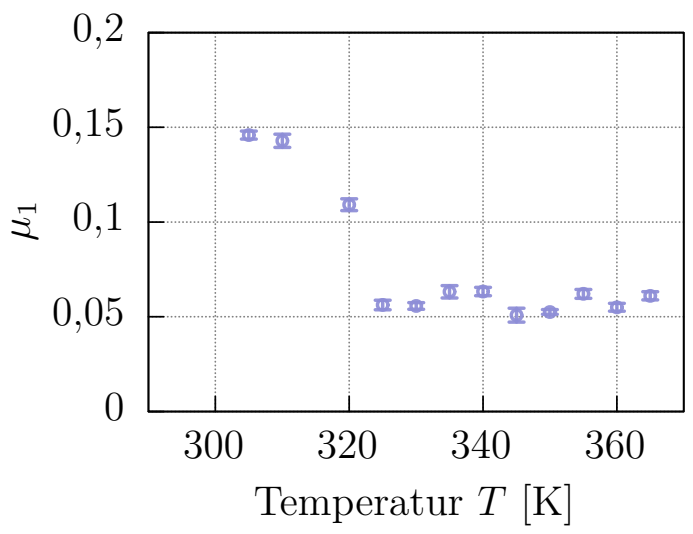

Abbildung 6.26 - Der Reibungskoeffizient $\mu_{1}$ der Deckschicht a) im Verhältnis zu $\mu_{2}$ zeigt ein Minimum bei $T \approx 325 \mathrm{~K}$. b) $\mu_{1}$ entspricht bei Raumtemperatur dem BulkWert und fällt dann auf einen Sättigungswert von $\approx 0,05$ bei $T \approx 325 \mathrm{~K}$.

Tiefe von [Johnson85]

$$
d_{\max } \approx 0,48 a_{\mathrm{c}}=0,48 \sqrt[3]{\frac{3}{4} \frac{\Delta F_{\mathrm{N}, \mathrm{on}} R}{E^{*}}}
$$

wobei die Probenoberfläche bei $d=0$ liegt. Über den ermittelten Amplitudenwert $A_{0}$ kann mit Gleichungen 6.6 und 6.12 die mittlere Normalkraft $\Delta F_{\mathrm{N} \text {,on }}$ unter externer Anregung bestimmt und damit die Tiefe $d_{\max }$ berechnet werden. Abbildung 6.28 zeigt die berechneten Werte $d_{\text {max }}$, wobei nur die Werte $A_{0}$ verwendet worden sind, bei denen die Anpassung an Gleichung 6.19 möglich ist (Abweichung vom quadratischen Verhalten). In Abbildung 6.28 ist zu erkennen, dass die Tiefe $d_{\max }$ in der Größenordnung von fünf LSMO-Einheitszellen liegt und leicht mit steigender Temperatur zunimmt. 
a) Mittelwertamplitude $A_{0}$

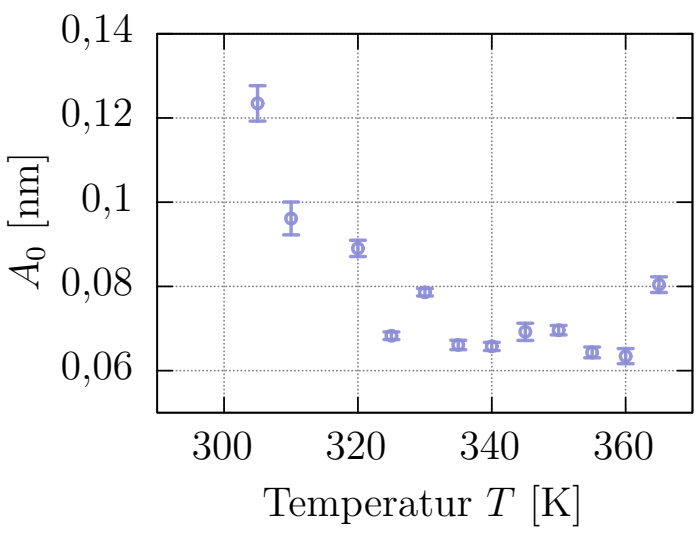

b) Breite $b$

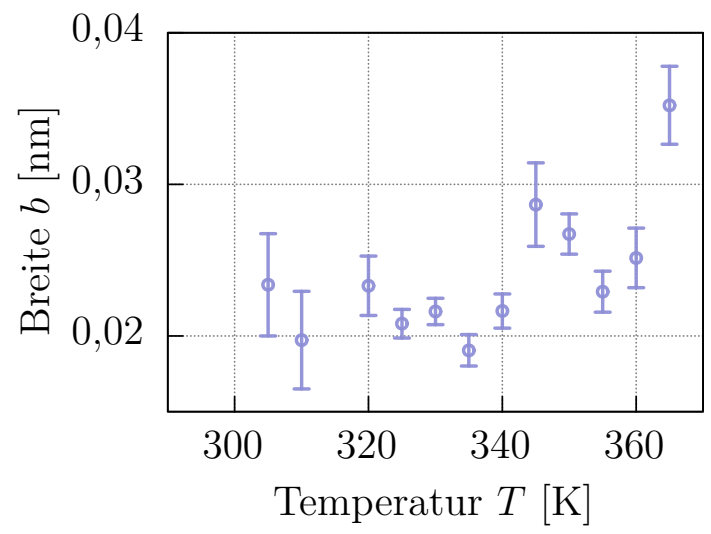

Abbildung 6.27 - Mittelwertamplitude $A_{0}$ und Breite b. a) Die Mittelwertamplitude $A_{0}$ wird kleiner mit steigender Temperatur. Die Breite $b$ steigt bei hohen Temperaturen an.

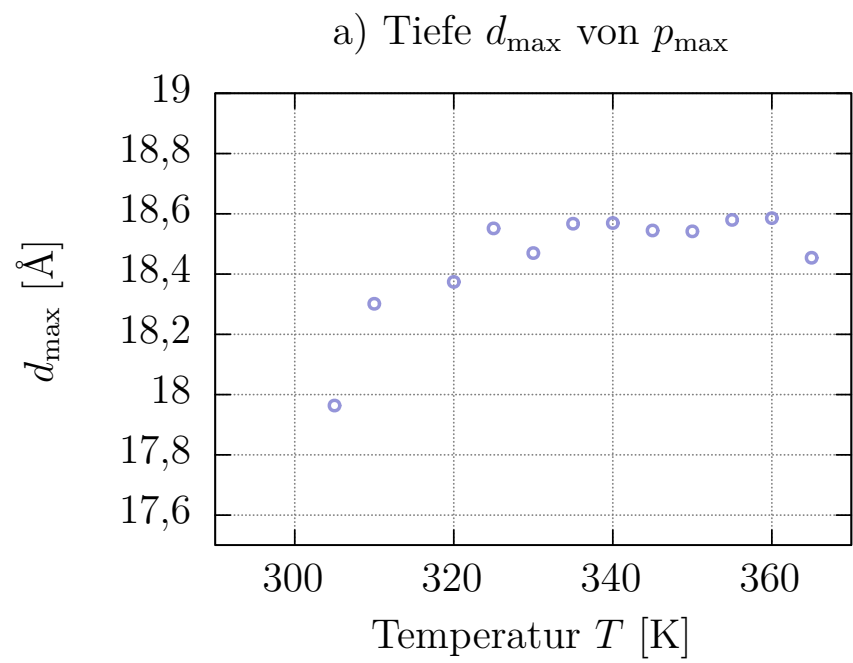

Abbildung 6.28 - Die Amplitudenwerte $A_{0}$ liefern mit Gleichungen 6.6 und 6.12 die mittlere Normalkraft $\Delta F_{N, \text { on }}$, mit der die Tiefe $d_{\max }$ der maximalen Schubspannung $p_{\max }$ berechnet werden kann. Die Tiefe $d_{\max }$ nimmt leicht mit steigender Temperatur zu. 


\title{
7 KONTAKTRESONANZ- REIBUNGSKRAFTMIKROSKOPIE
}

In Kapitel 6 sind FFM-Reibungsmessungen mit und ohne externe Anregung durchgeführt worden. In diesem Kapitel wurde auch gezeigt, dass hohe Anregungsamplituden dazu führen können, dass die Kontaktresonanzkurven die Form einer CauchyLorentzkurve verlieren und chaotisches Verhalten zeigen (vgl. Abb. 6.3). Das geschieht, wenn die Blattfeder so starke Verbiegung erfährt, dass die Spitze die statische Reibung des Kontakts überwindet und anfängt, auf der Oberfläche hin und her zu gleiten.

\begin{abstract}
Abbildung 7.1 - Wird die Spitze im Kontakt angeregt, bildet sich im äußeren Bereich eine Kreisringfläche aus, die auf der Oberfläche reibt. Dies soll zur Untersuchung der Reibung verwendet werden. Nach [Mazeran08].
\end{abstract}

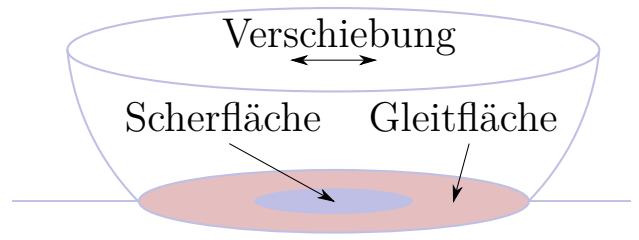

Im Bereich zwischen statischem Kontakt ohne Anregung und dem Loslösen der Spitze bei zu starken Anregungen kommt es zum sogenannten Micro-Slip-Verhalten, bei dem sich nur ein Teil der Spitze auf der Oberfläche bewegt. Abbildung 7.1 zeigt dies illustriert. Ein Teil der Dämpfung der Blattfederoszillation stammt dann aus dem Bereich der Gleitfläche. Im Folgenden wird dieses Verhalten zur Untersuchung der Gleitreibung verwendet. Es soll Kontaktresonanz-FFM (CR-FFM) ${ }^{1}$ genannt werden. Die Evaluation der Kontaktresonanzdaten soll zunächst anhand der $\mathrm{La}_{1-x} \mathrm{Sr}_{x} \mathrm{MnO}_{3}$-(LSMO)-Probe in Abhängigkeit von der Temperatur gezeigt werden. Die Probe liegt daher auf der Heizstation und ist zur besseren Wärmekopplung mit Leitsilber auf die Heizstation aufgeklebt. Daher wird hier der eingebaute Tapping-Piezo zur Anregung verwendet. Wie im vorherigen Kapitel beschrieben wurde, könnte für das CR-FFM daher prinzipiell auch ein an der Probe angebrachter Schallwandler verwendet werden. Zum Vergleich werden im Anschluss Ergeb-

\footnotetext{
${ }^{1}$ engl. Contact Resonance Friction Force Microscopy
} 
nisse der $\mathrm{Pd}_{77,5} \mathrm{Cu}_{6} \mathrm{Si}_{16,5}$-Proben dargestellt. Folgende Ergebnisse sind teilweise in [Pfahl17] veröffentlicht und dementsprechend gekennzeichnet.

\subsection{Auswertung}

Im CR-FFM-Modus wird die Blattfeder im Kontakt mittels Tapping-Piezo zu Biegeschwingungen angeregt. Die Blattfederamplitude wird dann über das vertikale Signal des Vier-Quadrant-Fotodetektors detektiert. Das Kontaktresonanzspektrum dient gemäß Kapitel 3.3.3 dazu, die komplexe Kontaktsteifigkeit $k^{*}$ zu bestimmen. Bei viskoser Dämpfung gilt für den Imaginärteil der Kontaktsteifigkeit $k_{i}^{*}=\omega_{n \text {,cont }} \gamma$ mit lokalem Dämpfungsterm $\gamma$. Zur Erinnerung wird Gleichung 3.21 hier wiederholt:

$$
Q_{\mathrm{loc}}^{-1}=\frac{E^{\prime \prime}}{E^{\prime}}=\frac{\omega_{n, \mathrm{cont}} \gamma}{k_{r}^{*}}
$$

Im Folgenden soll anhand einer Messung auf der LSMO-Probe bei $300 \mathrm{~K}$ der Verlauf der Datenauswertung dargestellt und die berechneten Parameter gezeigt werden.

\subsubsection{RESONANZKURVEN}

Zur Untersuchung der elastischen Eigenschaften werden Position und Breite der Kontaktresonanzkurven mit denen der Resonanzkurve der freien Blattfeder verglichen.

\section{FREIE BLATTFEDER}

Abbildung 7.2 zeigt beispielhaft eine gemessene freie Resonanzkurve der verwendeten Blattfeder und die zugehörige Anpassung an eine Cauchy-Lorentz-Verteilung (Lorentzkurve) der Form

$$
C=a\left[\left(1-\left(\frac{x}{f_{\text {free }}}\right)^{2}\right)^{2}+\left(\frac{x}{f_{\text {free }}} Q_{\text {free }}^{-1}\right)^{2}\right]^{-1 / 2}+b
$$

wobei $a, b, f_{\text {free }}$ und $Q_{\text {free }}$ Fit-Parameter sind. Im Beispiel der Abbildung 7.2 ergibt sich für die Kontaktresonanzfrequenz $f_{\text {free }} \approx 172,6 \mathrm{kHz}$ und die Güte bzw. Qualität 
der Kurve $Q_{\text {free }}^{-1} \approx 425$ und man erkennt, dass die Lorentzkurve eine sehr gute Übereinstimmung mit der Messung darstellt.

Abbildung 7.2 - An die Resonanzkurve der freien Blattfeder wird eine Lorentzverteilung angefittet. Dadurch erhält man die Werte $f_{\text {free }} \approx 172,6 \mathrm{kHz}$ und $Q_{\text {free }}^{-1} \approx 425$.

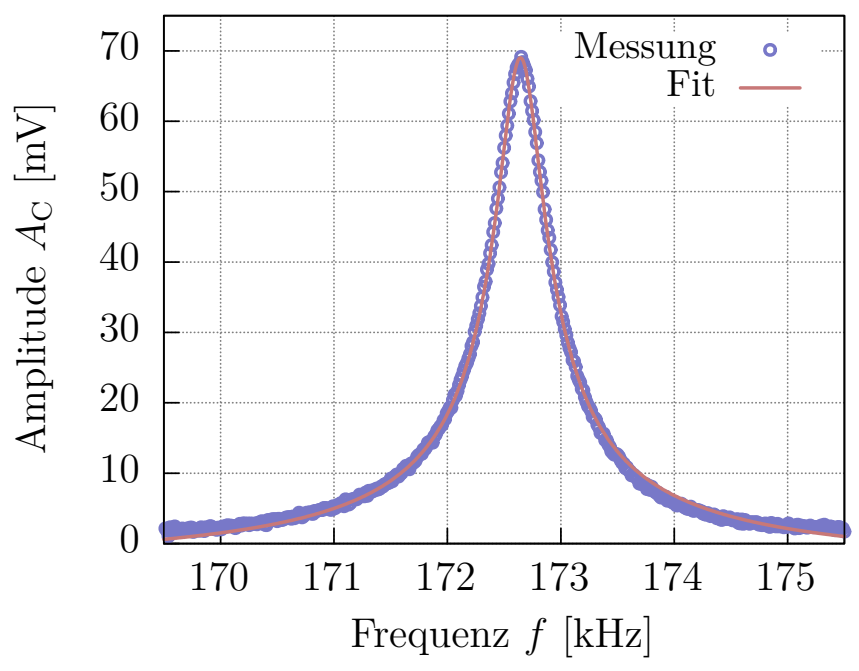

\section{IM KONTAKT}

Wie schon in Kapitel 6 muss auch in diesem Experiment sichergestellt werden, dass sich die Blattfeder nicht von der Oberfläche löst. Dies ist unter anderem daran zu erkennen, dass die Kontaktresonanzkurve von einer Lorentzkurve abweicht. Dazu sind in Abbildung 7.3 a) die Amplituden, b) der Auslenkungsfehler ${ }^{2}$ und c) die Signale des Höhensensors von zwei Frequenzsweeps in unterschiedlichen Richtungen also von niedrigen zu hohen Frequenzen (blau) und andersherum (rot) - dargestellt.

In AFM-Messungen im Kontakt wird eine gewünschte Normalkraft eingestellt, indem über eine Kraft-Abstands-Kurve die zugehörige Blattfederauslenkung bestimmt wird, die dann in der Software als Sollwert ${ }^{3}$ eingestellt wird. Die Rückkopplung überprüft während einer Messung die Auslenkung der Blattfeder und passt sie gegebenenfalls über Verfahren des z-Piezos an. Dazu berechnet es den Auslenkungsfehler, der die Differenz zwischen der tatsächlichen Blattfederauslenkung und dem Sollwert angibt.

Bei beiden Resonanzkurven in Abbildung 7.3 b) ist zu erkennen, dass bei ausreichend großen Amplituden der Fehler der Auslenkung ausschlägt. Das führt dazu, dass die Rückkopplung des AFM eingreift und die Auflagekraft anpasst. Dies wird auch am Signal des Höhensensors deutlich. Das negative Signal bedeutet, dass die

\footnotetext{
${ }^{2}$ engl. Deflection Error

${ }^{3}$ engl. Deflection Setpoint
} 
a) Amplitude

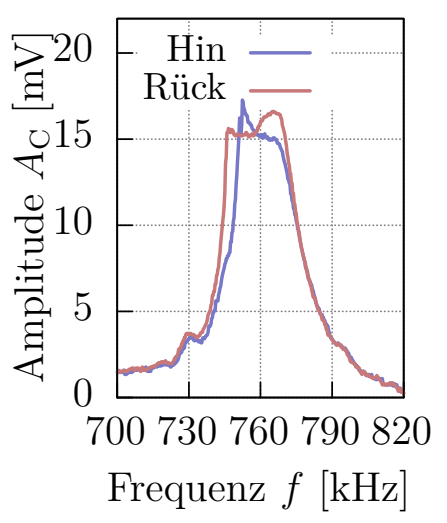

b) Auslenkungsfehler

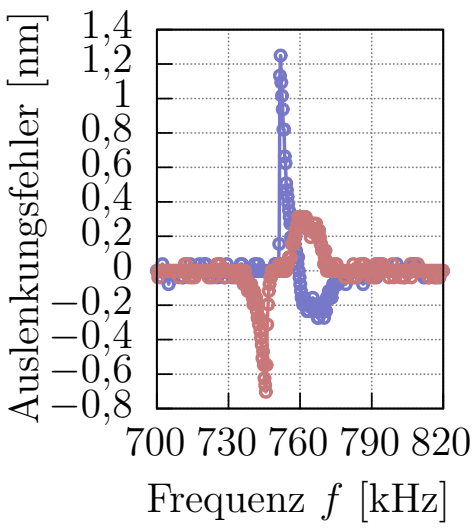

c) Höhensensor

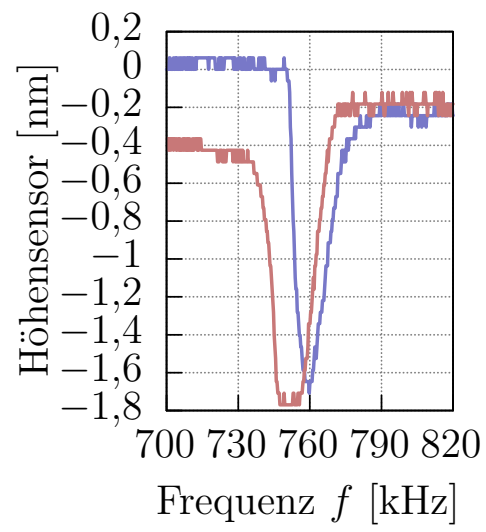

Abbildung 7.3 - Zu starke Anregungen führen zum Eingreifen der Rückkopplung. Das äußert sich in der Form der a) Amplitude, b) dem Deflection Error und daher auch in der Form des c) Höhensignals.

Spitze näher an die Oberfläche herangeführt wird. Dadurch ändert sich neben der Auflagekraft auch die Kontaktsteifigkeit, die wiederum Einfluss auf die Resonanzkurve selbst hat.

Es muss also sichergestellt werden, dass die Kontaktresonanzkurven die Form einer Lorentzkurve beibehalten. Es gibt mehrere Möglichkeiten, dies zu erreichen: Zum einen kann man die Anregungsamplitude gering halten. Zum anderen können hohe Auflagekräfte verwendet werden, um die Spitze an die Probe zu pressen. Um einen möglichst großen Messbereich nutzen zu können, bieten sich also kurze Blattfedern an, die hohe Steifigkeiten bzw. Federkonstanten aufweisen.

Abbildung 7.4 zeigt den Vergleich zwischen einer weichen Blattfeder mit $k_{\mathrm{c}}=$ $4,36 \mathrm{~N} \mathrm{~m}^{-1}$ bei der Anregungsamplitude $A_{\mathrm{D}}=30 \mathrm{mV}$ für 19 verschiedene Normalkräfte von $F_{\mathrm{N}}=87 \mathrm{nN}$ bis $3,1 \mu \mathrm{N}$ und einer härteren mit $k_{\mathrm{c}}=48 \mathrm{~N} \mathrm{~m}^{-1}$ bei der Anregungsamplitude $A_{\mathrm{D}}=10 \mathrm{mV}$ im Kräftebereich von $F_{\mathrm{N}}=480 \mathrm{nN}$ bis $43,2 \mu \mathrm{N}$. Man erkennt, dass die Kontaktresonanzen der harten Blattfeder aus Abbildung 7.4 b) - im Gegensatz zur weichen Blattfeder - über den gesamten Kräftebereich lorentzförmig bleiben. Die verwendete Software (s. Kap. 7.1.2) kann diese Kurven schließlich auswerten.

Große Federkonstanten führen zu hohen Auflagekräften. Dies wiederum kann zu sehr hohen Drücken führen. Die genannte Normalkraft von $F_{\mathrm{N}}=43,2 \mu \mathrm{N}$ beispielsweise führt bei typischen Kontaktradien neuer Spitzen von $\leq 5 \mathrm{~nm}$ zu Drücken von 
a) weiche Blattfeder

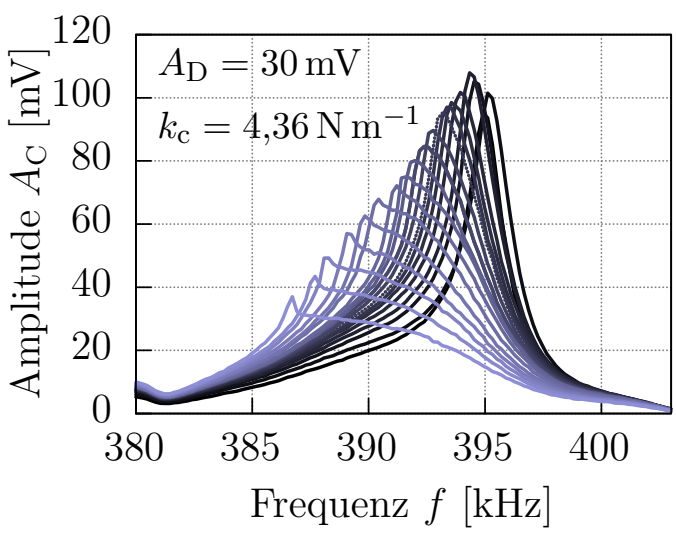

b) harte Blattfeder

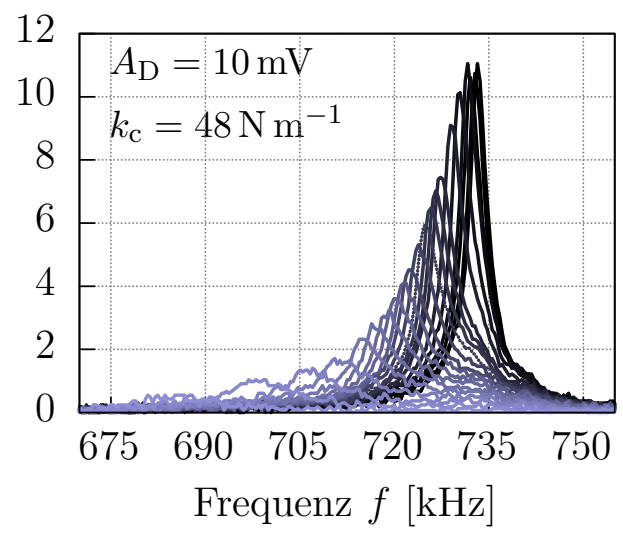

Abbildung 7.4 - Kontaktresonanzkurven bei unterschiedlicher Normalkraft für zwei Blattfedern mit a) $k_{c}=4,36 \mathrm{~N} \mathrm{~m}^{-1}$ und b) $k_{c}=48 \mathrm{~N} \mathrm{~m}^{-1}$ auf der LSMO-Probe. Weichere Blattfedern zeigen bei kleinen Normalkräften ein deutliches Abweichen von einer Lorentzkurve. Harte Blattfedern eignen sich daher besser für diese Messmethode, da im gesamten Kräftebereich Lorentzkurven gemessen werden.

$\geq 550 \mathrm{GPa}$. Dieser Wert liegt fern jeglicher kritischer Schubspannungen und würde zur Zerstörung der Spitze oder der Probe führen. Um diese Drücke zu mindern, muss die Kontaktfläche vor den Messungen daher vergrößert werden. Das wird umgesetzt, indem die Spitze mechanisch abgerieben wird.

Abbildung 7.5 zeigt eine verwendete Messspitze mit $k_{\mathrm{c}}=48 \mathrm{~N} \mathrm{~m}^{-1}$. Der Radius beträgt hier etwa $198 \mathrm{~nm}$. Nimmt man an, dass die Spitze zylinderförmig ist, führt dies zu maximalen Drücken von etwa 0,35 GPa. Unter der Annahme, dass die Spitze kugelförmig ist, gilt für die Kontaktfläche mit der LSMO-Probe nach [Johnson85]

$$
a_{\mathrm{c}}=\sqrt[3]{\frac{3}{4} \frac{F_{\mathrm{N}} R}{E^{*}}} \approx 54 \mathrm{~nm},
$$

die zu maximalen Drücken von 4,7 GPa führen würde. Die Aufnahme der Spitze macht aber deutlich, dass der Kontakt keinesfalls durch einen Kontakt zwischen Kugel und Ebene beschrieben werden kann. Die wahre Kontaktfläche ist aufgrund der Krümmung der Spitze dennoch kleiner als im zylinderförmigen Fall. 


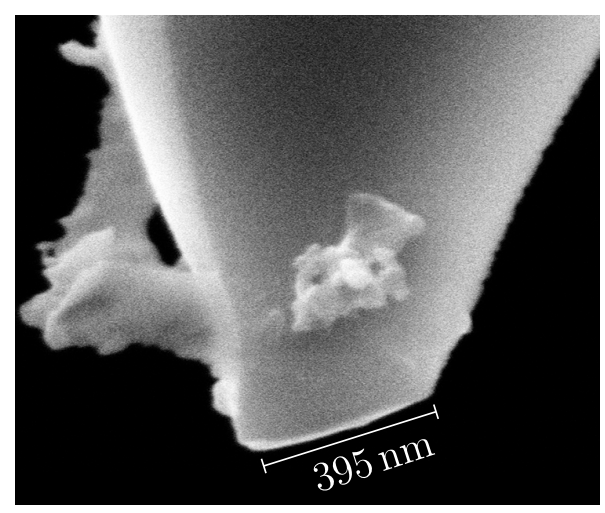

Abbildung 7.5 - Bevor eine Blattfeder verwendet wird, wird sie glatt gerieben, um die Drücke auf die Probe zu verringern. Der Radius vergrößert sich um 1-2 Größenordnungen im Vergleich zur neuen Blattfeder. Die SEMAufnahme zeigt, dass die Form der Blattfeder noch nicht zylinderförmig ist. Die tatsächliche Kontaktfläche ist daher geringer als der gemessene Radius von $198 \mathrm{~nm}$.

\subsubsection{PARAMETERBERECHNUNG}

Die Berechnung der Parameter erfolgt automatisiert mit einem LabView-Programm, das am I. Physikalischen Institut der Universität Göttingen entwickelt worden ist. Die Berechnungen basieren dabei auf dem Kontaktresonanzmodell aus Kapitel 3.3.3. Folgende Daten sind aus den Messkurven aus Abbildung 7.4 b) generiert worden.

Über eine Anpassung an die Funktion 7.1 werden zunächst die Frequenz $f_{0}$ und die zugehörige Amplitude $A_{\mathrm{C}}$ sowie der Qualitätsfaktor $Q$ aus den Kontaktresonanzkurven bestimmt. Daraus können schließlich der Realteil der Kontaktsteifigkeit $k_{\mathrm{r}}^{*}$ und die Dämpfung $\gamma$ berechnet werden. Abbildung 7.6 zeigt die Daten der LSMO-Probe bei $300 \mathrm{~K}$ in Abhängigkeit von der Normalkraft $F_{\mathrm{N}}$.

Abbildung 7.7 zeigt schließlich die normierte Dämpfung $\gamma / k_{\mathrm{c}}$ in Abhängigkeit von der Kontaktsteifigkeit der LSMO-Probe bei $300 \mathrm{~K}$. 
a) Kontaktresonanzfrequenz

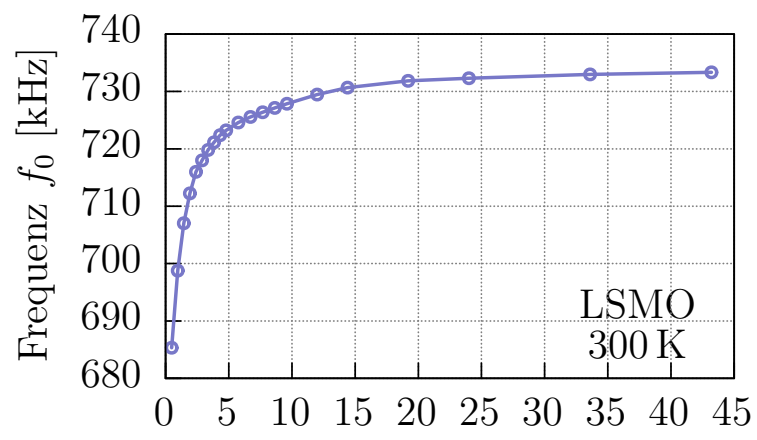

c) Qualitätsfaktor $Q$

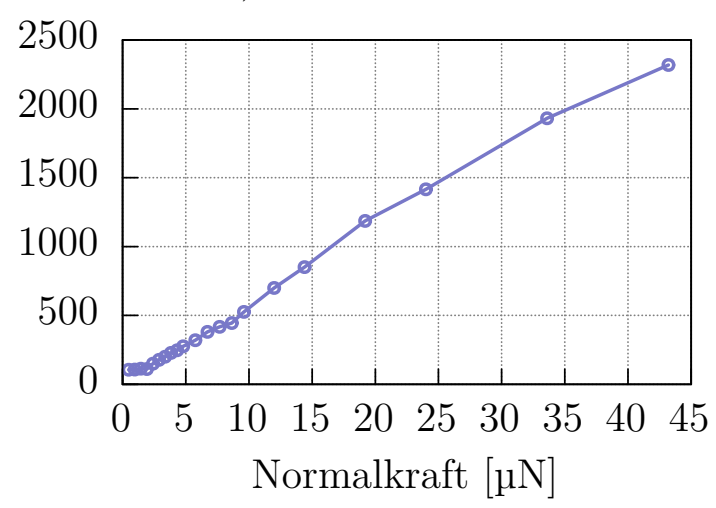

b) Kontaktresonanzamplitude

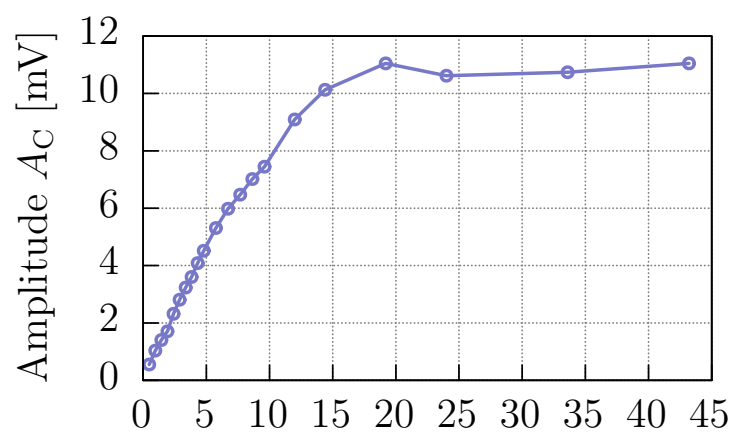

d) Realteil der Kontaktsteifigkeit

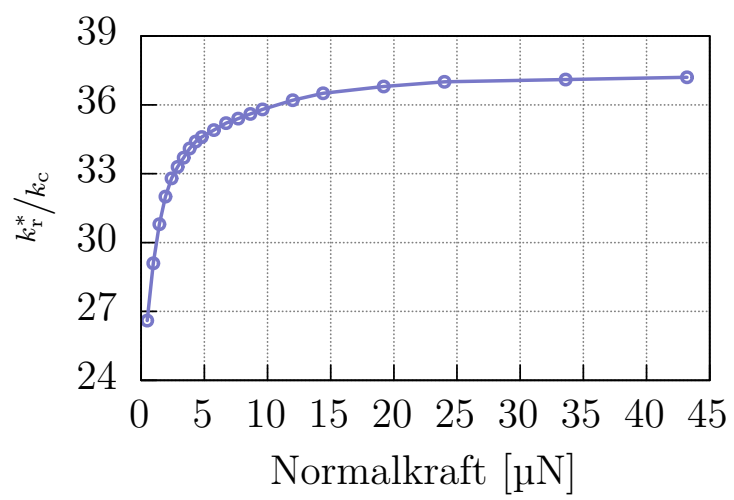

Abbildung 7.6 - Aus der Anpassung der Resonanzkurven der LSMO-Probe bei $300 \mathrm{~K}$ werden a) die Kontaktresonanzfrequenz $f_{0}$, b) die Amplitude $A_{C}$ und c) der Qualitätsfaktor $Q$ bestimmt. Damit kann die normierte Kontaktsteifigkeit $k_{r}^{*} / k_{c}$ d) berechnet werden. Die Daten sind hier in Abhängigkeit von der Normalkraft $F_{N}$ dargestellt.

Abbildung 7.7 - Die normierte Dämpfung $\gamma / k_{c}$ der LSMOProbe bei $300 \mathrm{~K}$ in Abhängigkeit von der normierten Kontaktsteifigkeit $k_{r}^{*} / k_{c}$.

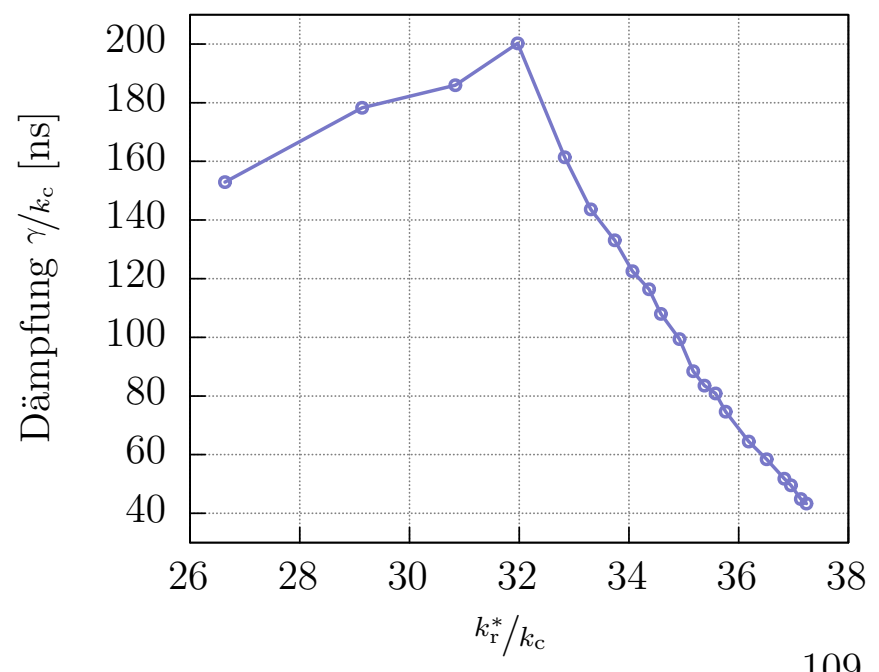




\subsection{ERGEBNISSE}

Es sind Kontaktresonanzkurven für verschiedene Temperaturen in $5 \mathrm{~K}$-Schritten auf amorphen und kristallinem $\mathrm{Pd}_{77,5} \mathrm{Cu}_{6} \mathrm{Si}_{16,5}$ und auf der $\mathrm{La}_{1-x} \mathrm{Sr}_{x} \mathrm{MnO}_{3}$-Dünnschicht aufgenommen worden. Die Auswertung dieser Daten verläuft identisch zum vorherigen Beispiel.

\subsubsection{LSMO}

Abbildung 7.8 zeigt die ermittelte normierte Dämpfung $\gamma / k_{\mathrm{c}}$ der LSMO-Probe in Abhängigkeit von der normierten Kontaktsteifigkeit $k_{\mathrm{r}}^{*} / k_{\mathrm{c}}$ für alle Temperaturen. Gestrichelte Linien stellen jeweils die Messungen dar, die bei $5 \mathrm{~K}$ höheren Temperaturen durchgeführt wurden als die durchgezogenen Linien der gleichen Farbe. Der Wert kleinster Kontaktsteifigkeit entstammt der Messung bei kleinster Auf-

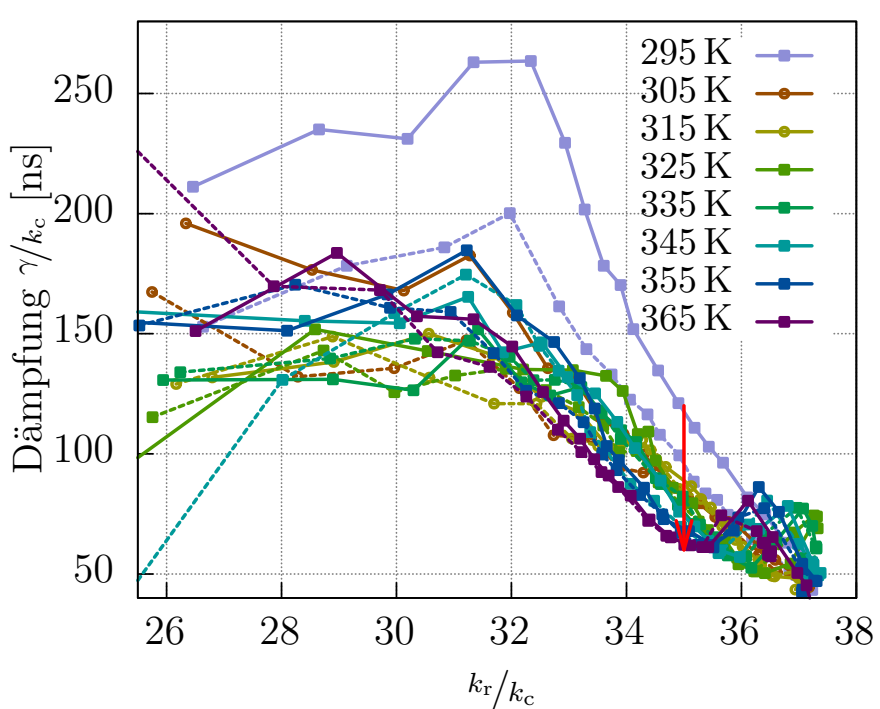

Abbildung 7.8 - Aus den Resonanzkurven ermittelte normierte Dämpfung in Abhängigkeit von der normierten Kontaktsteifigkeit $k_{r}^{*} / k_{c}$ für verschiedene Temperaturen in $5 \mathrm{~K}$ Schritten. Gestrichelte Linien entsprechen $5 \mathrm{~K}$ höheren Temperaturen als Linien der gleichen Farbe. Die Normalkraft liegt zwischen $F_{N, \min } \approx 480 \mathrm{nN}$ und $F_{N, \max } \approx 43,2 \mu \mathrm{N}$.

lagekraft von $F_{\mathrm{N}, \min } \approx 480 \mathrm{nN}$. Mit steigender Normalkraft steigt auch die Kontaktsteifigkeit. Die höchste Kontaktsteifigkeit entspricht hier einer Normalkraft von $F_{\mathrm{N} \text {,max }} \approx 43,2 \mu \mathrm{N}$. Es ist zu erkennen, dass die Dämpfung für alle Temperaturen zunächst konstant bleibt oder ansteigt. Ab $k_{r} / k_{c} \approx 32$ fällt sie dann linear bis $k_{r} / k_{c} \approx 35$ ab. Anschließend kann man für höhere Temperaturen ein lokales Maximum erkennen

Um die Dämpfung in Abhängigkeit von der Temperatur zu untersuchen, wird sie bei konstanter Kontaktsteifigkeit für jede Temperatur aus Abbildung 7.8 bestimmt. Der Weg, den beispielsweise der rote Pfeil beschreibt, entspricht der Dämpfung bei $k_{r} / k_{c}=35$, was einer Normalkraft von $F_{\mathrm{N}}=5,76 \mu \mathrm{N}$ bis $F_{\mathrm{N}}=8,16 \mu \mathrm{N}$ entspricht. 
Abbildung 7.9 - Die normierte Dämpfung $\gamma / k_{c}$ der LSMOProbe bei konstanter normierter Kontaktsteifigkeit $k_{r} / k_{c}=35$ nimmt mit der Temperatur ab. Nach [Pfahl17].

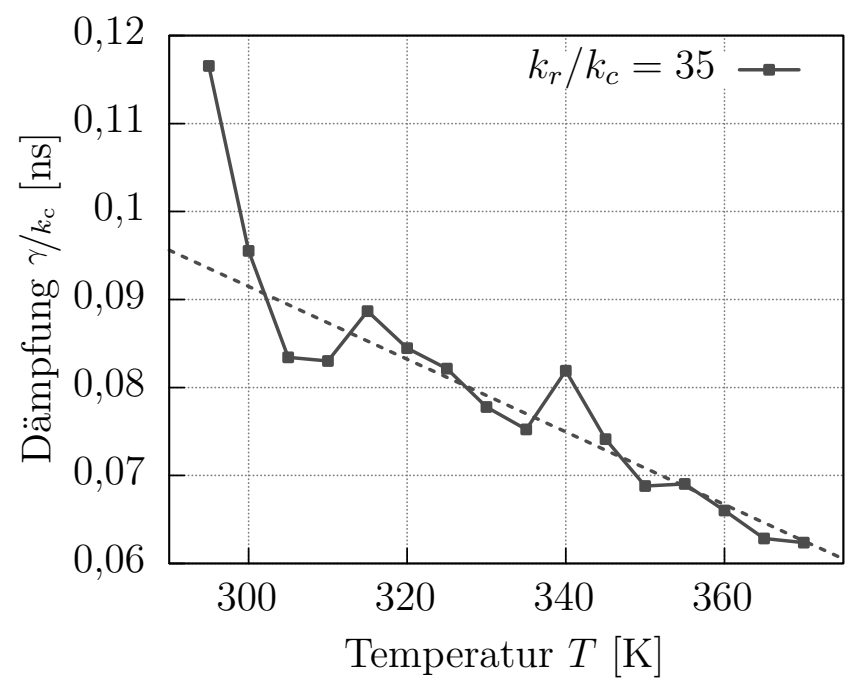

Abbildung 7.9 zeigt die ermittelten Werte für diese Kontaktsteifigkeit. Die Dämpfung nimmt über den gesamten Temperaturbereich um etwa $45 \%$ ab, dabei scheint die Abnahme bis auf den Wert bei Raumtemperatur linear zu sein, wie die gestrichelte Linie andeutet.

Abbildung 7.10 zeigt den Verlauf der normierten Dämpfung für weitere Kontaktsteifigkeiten $k_{r} / k_{c} \geq 28$. Bis zu einer Temperatur von $T \approx 315 \mathrm{~K}$ fällt die Dämpfung für alle Kontaktsteifigkeiten ab. Bei Kontaktsteifigkeiten $k_{r} / k_{c} \leq 33$ beginnt die Dämpfung für höhere Temperaturen jedoch wieder anzusteigen. Daher ist im Bereich von $T \approx 325 \mathrm{~K}$ ein breites Minimum zu erkennen.

Abbildung 7.10 - Die normierte Dämpfung $\gamma / k_{c}$ der LSMOProbe für verschiedene normierte Kontaktsteifigkeiten. Bei niedrigen Kontaktsteifigkeiten ist ein Minimum um $T \approx 325 \mathrm{~K}$ zu erkennen.

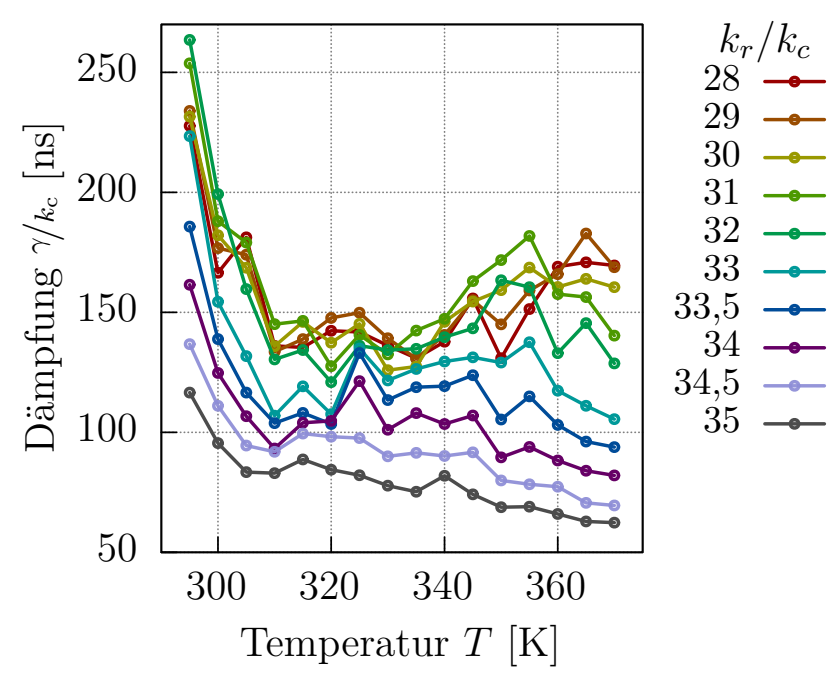




\subsubsection{AMORPHES PdCUSi}

Abbildung 7.11 zeigt die gleiche Auftragung der normierten Dämpfung für die amorphe Probe. Die gestrichelten Linien gehören wieder zu Messungen die bei $5 \mathrm{~K}$ höherer Temperatur durchgeführt wurden als die durchgezogenen Linien gleicher Farbe. Zusätzlich sind drei weitere Messreihen eingezeichnet, die beim Kühlprozess aufgenommen wurden. Sie werden durch gepunktete Linien und quadratische Symbole dargestellt. Im Rahmen der Messgenauigkeit liefern sie die gleichen Werte wie im Heizprozess. Man erkennt, dass die a-PdCuSi-Probe ein anderes Verhalten als die LSMO-Probe zeigt. Die Dämpfung zeigt hier mit steigender Kontaktsteifigkeit weder einen Bereich konstanter noch wieder ansteigender Dämpfung, sondern nur Bereiche mit unterschiedlich starkem Abfall. Auch lokale Extrema sind hier nicht zu erkennen. Im Temperaturbereich von $T \approx 310 \mathrm{~K}$ bis $340 \mathrm{~K}$ kann man eine Steigungsänderung bei $k_{r} / k_{c} \approx 44$ erkennen. Diese Steigungsänderung führt zu Maxima in Abbildung 7.12, die die normierte Dämpfung in Abhängigkeit von der Temperatur für verschiedene Kontaktsteifigkeiten zeigt.

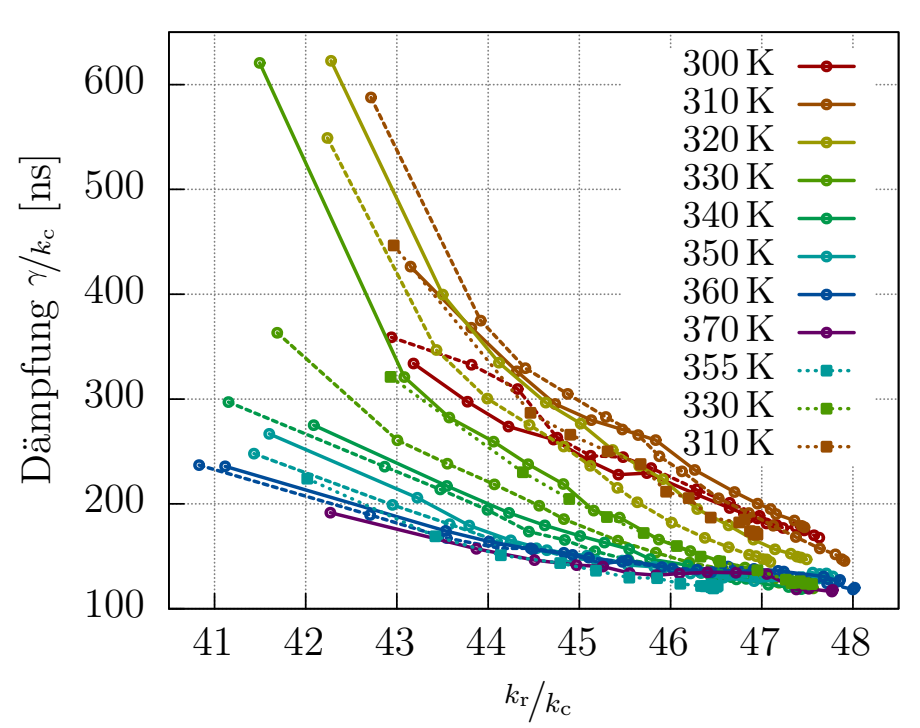

Abbildung 7.11 - Die normierte Dämpfung $\gamma / k_{c}$ von a-PdCuSi in Abhängigkeit von der normierten Kontaktsteifigkeit $k_{r}^{*} / k_{c}$ für verschiedene Temperaturen in $5 \mathrm{~K}-$ Schritten. Gestrichelte Linien entsprechen $5 \mathrm{~K}$ höheren Temperaturen als Linien der gleichen Farbe. Die Normalkraft liegt zwischen $F_{N, \min } \approx 960 \mathrm{nN}$ und $F_{N, \max } \approx 48 \mu \mathrm{N}$.

Für jede Kontaktsteifigkeit bildet sich hier ein Maximum aus, das bei hohen Kontaktsteifigkeiten von $k_{r} / k_{c} \approx 47$ bei etwa $T \approx 310 \mathrm{~K}$, bei niedrigeren Kontaktsteifigkeiten im Bereich von $T \approx 315 \mathrm{~K}$ liegt. 
Abbildung 7.12 - Die normierte Dämpfung $\gamma / k_{c}$ der a-PdCuSiProbe für verschiedene normierte Kontaktsteifigkeiten. Im Bereich von $T \approx 315 \mathrm{~K}$ sind Maxima zu erkennen.

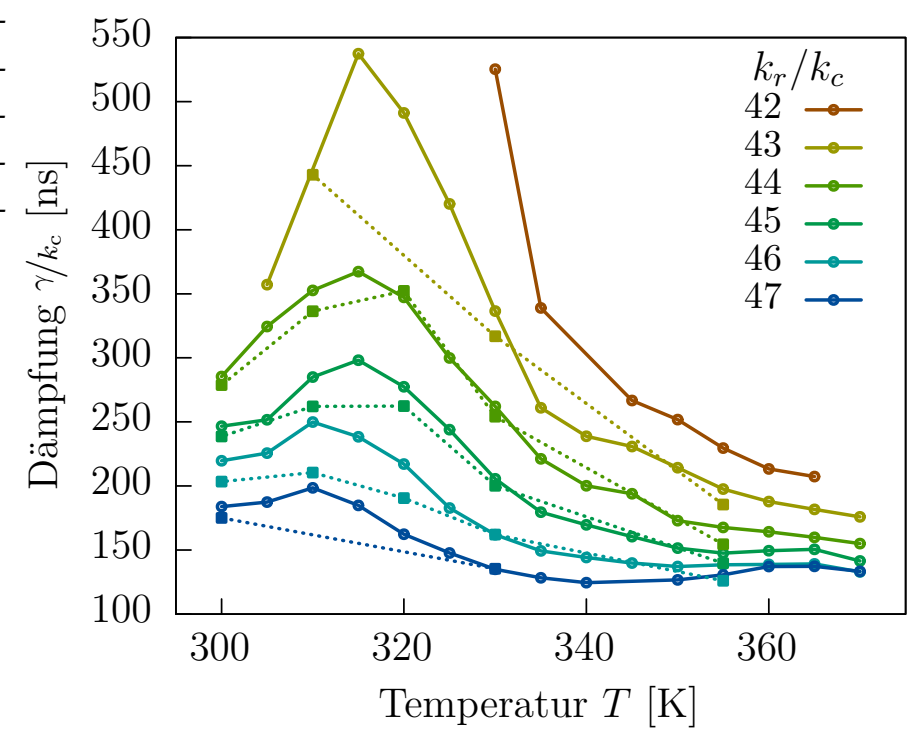

\subsubsection{KRISTALLines PdCuSi}

F-AFAM-Messungen auf der kristallinen PdCuSi-Probe zeigen wieder ein leicht anderes Ergebnis. Abbildung 7.13 zeigt die normierte Dämpfung gegen die Kontaktsteifigkeit aufgetragen. Auch hier ist im Gegensatz zur LSMO-Messung kein konstanter oder wieder ansteigender Bereich zu erkennen. Im Gegensatz zur amorphen Probe ist aber auch keine sprunghafte Steigungsänderung zu erkennen. Vor allem für Kontaktsteifigkeiten $k_{r} / k_{c} \geq 48$ liegen die Messkurven sehr nah beieinander. Auffällig ist auch, dass sämtliche Messpunkte, bei denen $k_{r} / k_{c} \leq 48$ gilt, von den Messungen bei den Temperaturen $T=350 \mathrm{~K}$ (blau gepunktet) und $T=310 \mathrm{~K}$ (braun gepunktet) - die beide während des Kühlprozesses aufgenommen wurden eingeschlossen werden.

Abbildung 7.13 - Die normierte Dämpfung $\gamma / k_{c}$ von $c-P d C u S i$ in Abhängigkeit von der normierten Kontaktsteifigkeit $k_{r}^{*} / k_{c}$ für verschiedene Temperaturen in $5 \mathrm{~K}$-Schritten. Gestrichelte Linien entsprechen $5 K$ höheren Temperaturen als Linien der gleichen Farbe. Die Normalkraft liegt zwischen $F_{N, \min } \approx 960 \mathrm{nN}$ und $F_{N, \max } \approx 48 \mu \mathrm{N}$.

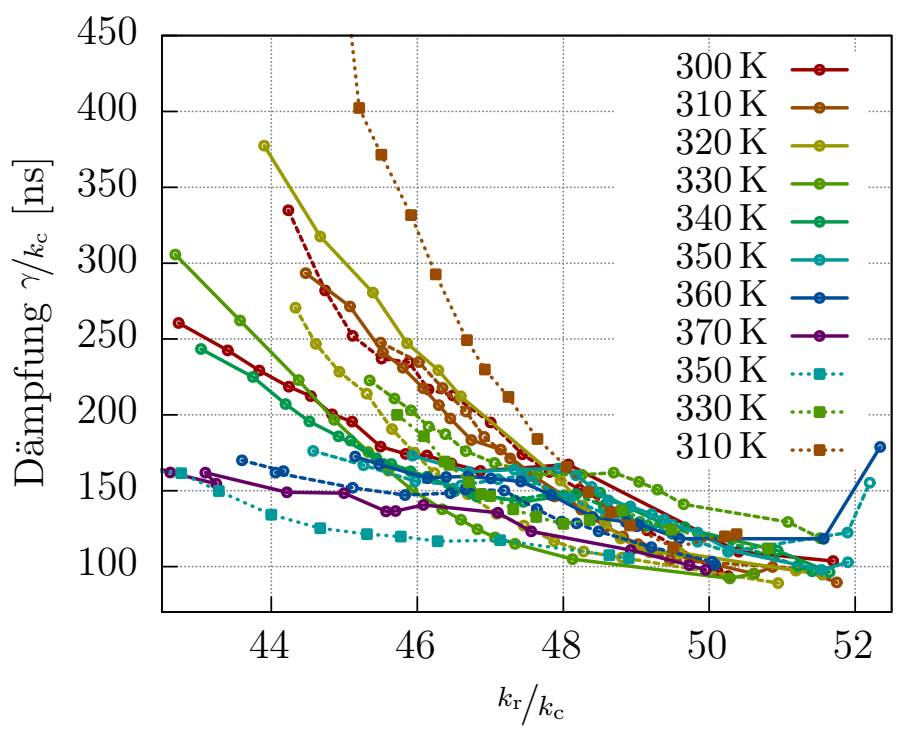




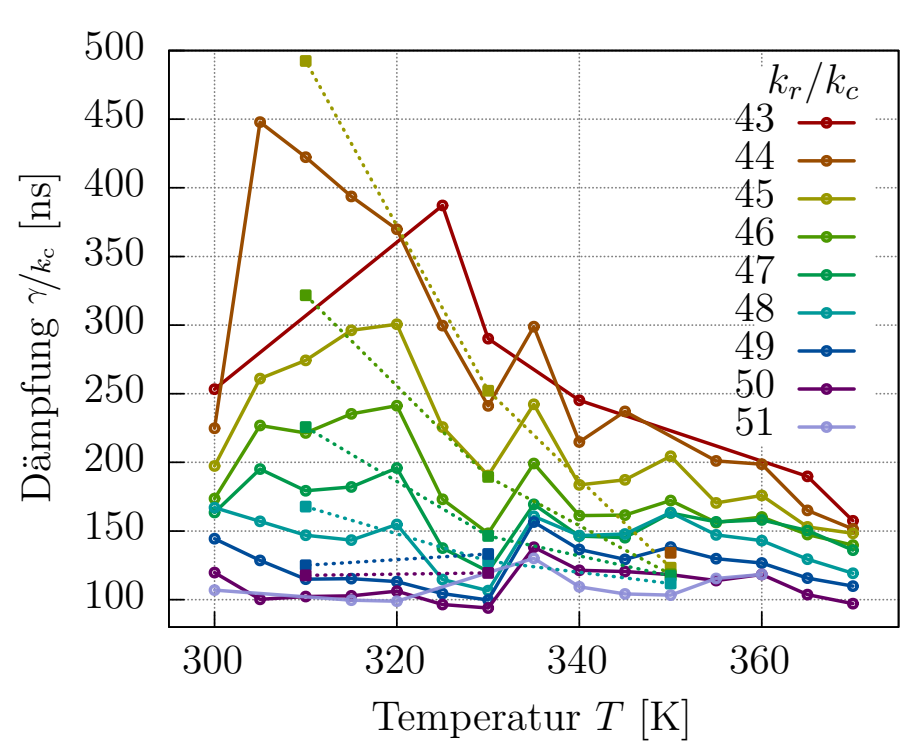

Abbildung 7.14 - Die normierte Dämpfung $\gamma / k_{c}$ der c-PdCuSiProbe für verschiedene normierte Kontaktsteifigkeiten.

Auch Abbildung 7.14 zeigt, dass die Messungen während des Kühlprozesses - im Gegensatz zur amorphen Probe - keine gute Übereinstimmung mit den Daten gleicher Temperatur beim Heizprozess aufweisen. Daher muss zunächst ein genauerer Blick in die Kontaktresonanzkurven geworfen werden. Es stellt sich heraus, dass diese Kurven bei kleinen Normalkräften - und daher auch niedrigen Kontaktsteifigkeiten - einen Doppelpeak aufweisen, was zu einem Fehler beim Fitten führt (vgl. Abb. 7.15). Ein Doppelpeak kommt üblicherweise vor, wenn die Spitze die Oberfläche der Probe an zwei Punkten berührt - also eine sogenannte Doppelspitze vorliegt ${ }^{4}$. Bei hohen Normalkräften wird er unterdrückt, da dann auch die Fläche zwischen beiden Spitzen die Probe erreicht. Außerdem wird die Spitze mit der Anzahl der Messungen mechanisch verändert und der kleinere Kontaktpunkt wird aufgrund der größeren Drücke abgerieben. Ab der Temperatur $T \approx 335 \mathrm{~K}$ treten hier keine Doppelpeaks mehr auf. Der entstandene Fehler äußert sich also vor allem bei den ersten Messungen - also bei niedrigen Temperaturen - und bei niedrigen Kontaktsteifigkeiten. Betrachtet man nur Messungen mit $T \geq 310 \mathrm{~K}$ und $k_{r} / k_{c} \geq 44$, ergibt sich Abbildung 7.16.

Es ist zu erkennen, dass die Dämpfung für alle Kontaktsteifigkeiten mit der Temperatur abnimmt. Außerdem gilt: Je niedriger die Temperatur, desto größer ist die Dämpfung bei niedrigen Kontaktsteifigkeiten. Das wird besonders deutlich, wenn man nur die Daten aus den Messungen während des Kühlprozesses betrachtet (gepunktete Linien).

\footnotetext{
${ }^{4}$ Die Spitze könnte auch unterschiedliche Orientierungen der polykristallinen Probe (s. Abschnitt 5.6.2) berühren, was zu mehreren Peaks führen würde [Wagner11b, Wagner11a].
} 
Abbildung 7.15 - Bei niedrigen Normalkräften und Temperaturen bilden sich Doppelpeaks aus, die zum Fehler im Fit führen. Dies muss berücksichtigt werden.

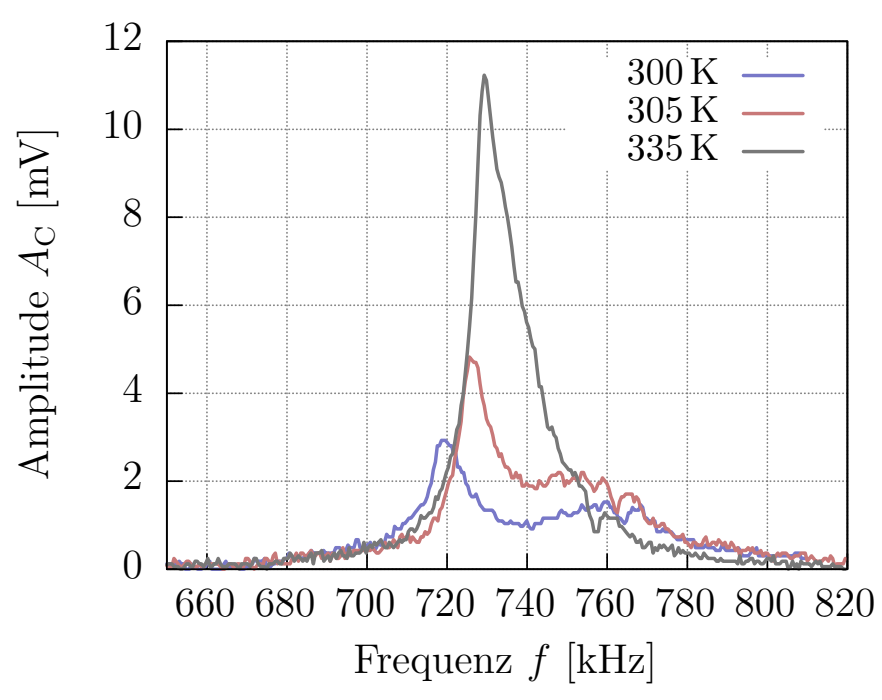

Abbildung 7.16 - Die normierte Dämpfung $\gamma / k_{c}$ der c-PdCuSiProbe für verschiedene normierte Kontaktsteifigkeiten. Fehlerbehaftete Werte sind entfernt worden. Die Dämpfung nimmt mit der Temperatur ab.

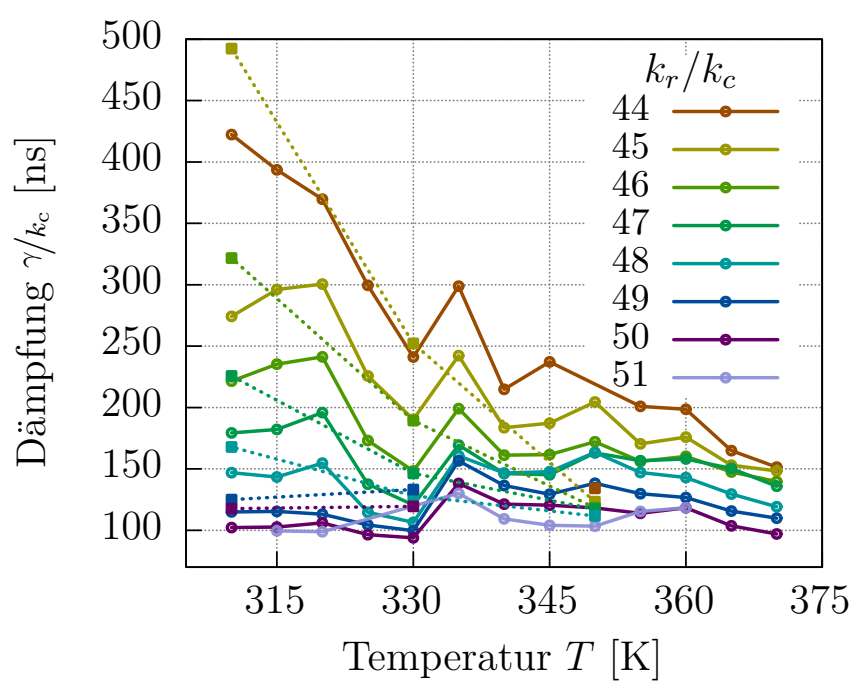





\section{DiskUssion}

In Kapitel 2 ist gezeigt worden, dass die Physik der Reibung äußerst komplex ist. Dazu kommt, dass Reibungsmessungen auch durch andere Faktoren beeinflusst werden. Dabei spielt vor allem der Kontakt selbst eine große Rolle. Punktförmige Einzelkontakte, die zum Beispiel in der Theorie des Prandtl-Tomlinson-Modell häufig verwendet werden, sind in Messungen nahezu unmöglich zu erreichen. Bei sehr kleinen Normalkräften ist die Kontaktfläche zwischen einer Messspitze und einer Probenoberfläche zwar klein, trotzdem erstreckt sie sich über mehrere Einheitszellen. Dass die Messspitze die Probe nur mit einem Atom berührt, ist nicht möglich.

Weiterhin spielt auch die Luftfeuchtigkeit eine Rolle. An Oberflächen setzt sich ein Wasserfilm ab, der die Reibung erhöhen oder mindern kann. Ohne kontrollierte Atmosphäre ist die Reibungsmessung mit einem Rasterkraftmikroskop im wahrsten Sinne des Wortes vom Wetter abhängig. Daher soll zu Beginn der Diskussion der Messergebnisse dieser Arbeit kurz auf den Einfluss der Luftfeuchtigkeit und der Spitze auf die Reibungsmessungen eingegangen werden.

Materialien zu untersuchen, die unterschiedliche Zustände besitzen können, kann hilfreich sein, um Reibung zu verstehen. Das Übergangsmetalloxid $\mathrm{La}_{1-x} \mathrm{Sr}_{x} \mathrm{MnO}_{3}$ $(x=0,4)$ ist dabei sehr informativ, da sich am Phasenübergang im Vergleich zu anderen Systemen mit ähnlichen Übergängen nur wenig ändert, so dass man eine Reibungsänderung einfacher mit der Änderung im System assoziieren kann. Da der Phasenübergang hier über die Temperatur getrieben wird, müssen auch thermische Einflüsse auf die Reibung abgeschätzt werden.

Auf der anderen Seite ist das untersuchte $\mathrm{Pd}_{77,5} \mathrm{Cu}_{6} \mathrm{Si}_{16,5}$-System interessant, da es im amorphen und kristallinen Zustand vorliegen kann. Chemisch unterscheiden sich diese Proben nicht, so dass Unterschiede in den Reibungsmessungen von der Struktur oder den Glaseigenschaften der amorphen Probe kommen müssen. 


\subsection{Allgemeines}

Bevor die physikalischen Prozesse der Reibung diskutiert werden können, muss abgeschätzt werden, ob äußere Einflüsse wie Luftfeuchtigkeit oder die verwendeten Spitzen die Ursache der gemessenen Reibungsänderungen sein können.

\subsubsection{Messspitze}

Die Kenntnis über die genaue Kontaktfläche zwischen Messspitze und Probenoberfläche ist zur Berechnung der herrschenden Drücke entscheidend. Allerdings ist es unmöglich, die Spitzenradien während einer Messung zu bestimmen. Der Aufwand wäre enorm, um nach jeder Reibungshysterese oder Dämpfungsmessung die Spitze erneut mit dem Rasterelektronenmikroskop (REM) zu vermessen, vor allem aber würde das Ein- und Ausbauen der Blattfeder Messergebnisse unvergleichbar machen, da durch das Verstellen des Lasers des AFM sämtliche Kalibrierungen unbrauchbar werden.

Daher ist es nur möglich, die Spitze nach einer Messung zu untersuchen und anzunehmen, dass der Spitzenradius während der Messung kleiner oder gleich dem war, den man im REM gemessen hat.

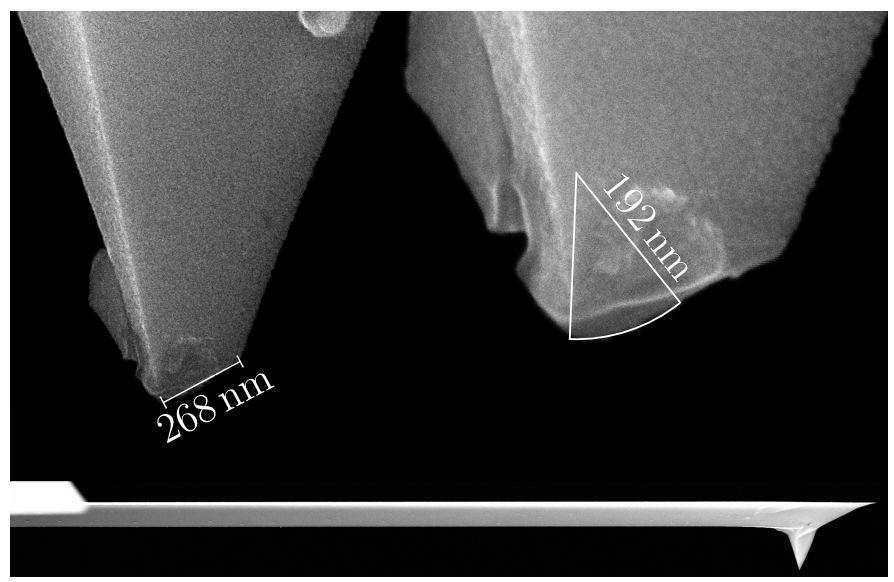

Abbildung $8.1 \quad-\quad R E M-$ Aufnahme der verwendeten Spitze. Der Radius der tatsächlichen Kontaktfläche muss zwischen $268 \mathrm{~nm}$ und dem Hertz'schem Radius $a_{c}$, der sich zwischen einer Kugel mit $R=192 \mathrm{~nm}$ und einer Ebene bildet, liegen.

Abbildung 8.1 zeigt die Spitze, die für die Messungen an den PdCuSi-Proben verwendet worden ist. Hier ist auch zu erkennen, dass Spitzen im CR-FFM-Modus durch das Abreiben nicht mehr als kugelförmig angesehen werden können. Die Form der Spitze liegt dabei zwischen der eines Zylinders und der einer Kugel.

Maximale Drücke werden durch kleinste Kontaktflächen erreicht. Andersherum führen große Kontaktflächen zu kleinen Drücken. Daher kann man anhand der REM-Aufnahmen den Bereich der möglichen Drücke eingrenzen. Die größtmögliche 
Kontaktfläche kann durch einen Zylinder mit Durchmesser $D \approx 268 \mathrm{~nm}$ beschrieben werden. Die kleinste Kontaktfläche entsteht, wenn man die Spitze als Kugel mit Radius $R=192 \mathrm{~nm}$ ansieht (vgl. jeweils Abb. 8.1).

Tabelle 8.1 zeigt, welche Drücke $P$ für die jeweilige Annahme im Beispiel der amorphen Probe entstehen. Dabei ist der Wert der Normalkraft $F_{\mathrm{N}}$ entsprechend der Messungen aus Kapitel 7 gewählt worden. Der Wert $a_{c}$ gibt den Radius der Kontaktfläche nach dem Hertz'schen Kontaktmodell an (vgl. Gl. 7.2, [Johnson85]). Man erkennt, dass der Druck $P_{\mathrm{K}}$ unter der Annahme einer Kugel um ein bis zwei Größenordnungen über dem Druck im Fall des Zylinders $P_{\mathrm{Z}}$ liegt.

Tabelle 8.1 - Drücke $P$ für eine kugelförmige $\left(P_{K}\right)$ bzw. zylinderförmige $\left(P_{Z}\right)$ Spitzenform.

\begin{tabular}{|c|cc|c|}
\hline$F_{\mathrm{N}}[\mathrm{\mu N}]$ & $a_{c}[\mathrm{~nm}]$ & $P_{K}[\mathrm{GPa}]$ & $P_{Z}[\mathrm{GPa}]$ \\
\hline \hline 0,96 & 13,21 & 1,75 & 0,017 \\
1,92 & 16,64 & 2,21 & 0,034 \\
2,40 & 17,92 & 2,38 & 0,043 \\
2,88 & 19,05 & 2,53 & 0,051 \\
3,36 & 20,05 & 2,66 & 0,060 \\
3,84 & 20,96 & 2,78 & 0,068 \\
4,32 & 21,80 & 2,89 & 0,077 \\
4,80 & 22,58 & 3,00 & 0,085 \\
5,76 & 24,00 & 3,18 & 0,102 \\
6,72 & 25,26 & 3,35 & 0,119 \\
7,68 & 26,41 & 3,50 & 0,136 \\
8,64 & 27,47 & 3,65 & 0,153 \\
9,60 & 28,45 & 3,78 & 0,170 \\
12,0 & 30,65 & 4,07 & 0,213 \\
24,0 & 38,61 & 5,12 & 0,426 \\
48,0 & 48,65 & 6,46 & 0,852 \\
\hline
\end{tabular}

\subsubsection{WASSER}

Der Einfluss von Wasser auf Reibungsexperimente ist komplex und kann den Reibungskoeffizienten um den Faktor 2 ändern [Piner97]. Auf Oberflächen adsorbiert ein Wasserfilm, der Menisken an der Messspitze ausbildet [Piner97]. Je nachdem, ob die Probenoberfläche hydrophil oder hydrophobisch ist, können die Menisken wie Kleber fungieren und die Reibung vergrößern [Piner97, Jinesh06] oder aber eine 


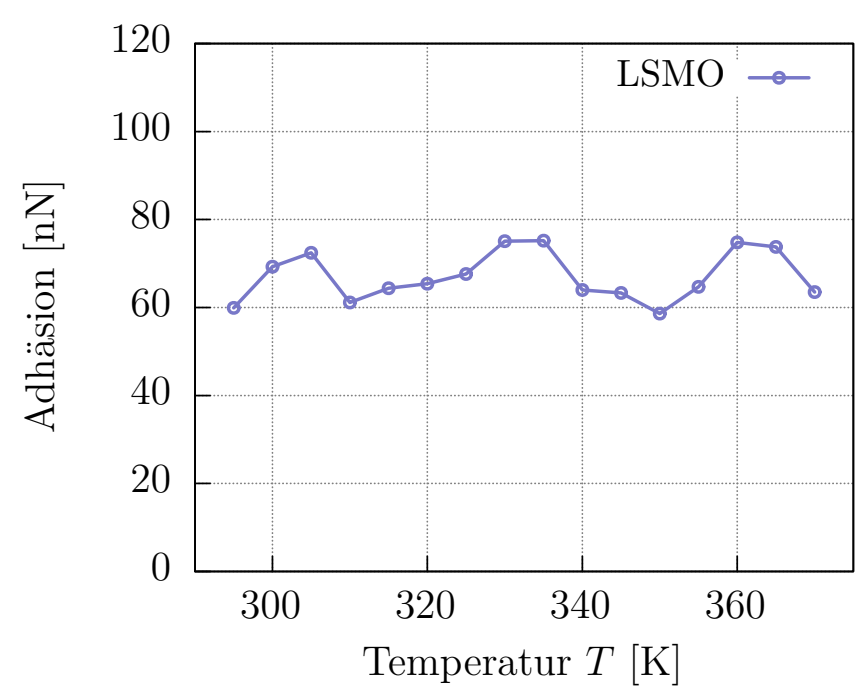

Abbildung 8.2 - Die Adhäsionskraft der LSMO-Messung zeigt zwar eine Varianz, aber kein Verhalten, das die gemessene Reibungsänderung erklären könnte.

schmierende Wirkung haben und so die Reibung vermindern [Binggeli94, Piner97, Tocha05].

Um den Einfluss von Wasser zu reduzieren, sind die Messungen in reduzierter Luftfeuchtigkeit durchgeführt worden. Das verwendete AFM steht in einer Kammer, die mit trockenem Stickstoff geflutet werden kann. Dadurch kann die Luftfeuchtigkeit auf $30 \%$ reduziert werden. Vor jeder Messung wurde die Luftfeuchtigkeit auf diesen Wert gebracht. Anschließend wurde etwa eine halbe Stunde gewartet, in der die Luftfeuchtigkeit wieder um etwa $10 \%$ ansteigt, da adsorbiertes Wasser auf allen Oberflächen innerhalb der Kammer abdampft. Ein weiteres Fluten führt dann zur gewünschten stabilen Luftfeuchtigkeit von etwa $30 \%$.

Scherge et al. [Scherge99] finden bei Luftfeuchtigkeiten unterhalb $30 \%$ auf hydrophilem Silizium im Temperaturbereich von 10 bis $80^{\circ} \mathrm{C}$ keinen Einfluss auf die Reibung. Hydrophobisches Silizium zeigt allerdings einen Anstieg der Reibungskraft mit steigender Luftfeuchtigkeit. Der Anstieg der Reibungskraft in Abhängigkeit von der Luftfeuchtigkeit ist aber für alle Temperaturen bis zur Luftfeuchtigkeit von etwa $50 \%$ konstant, so dass es keine Abhängigkeit von der Temperatur gibt.

Jinesh et al. [Jinesh06] zeigen, dass der Wassereffekt bei hydrophobischen Proben in den Reibungshysteresen zu erkennen ist. Ihrer Vermutung nach zieht die Spitze den Wasserfilm über die Probe, was zu einem Reibungspeak am Ende einer Reibungshysterese führt. Derartige Artefakte sind in den hier gezeigten Messungen nicht gefunden worden.

Abbildung 8.2 zeigt die Adhäsionskraft der LSMO-Probe in Abhängigkeit von der Temperatur. Der Wert wird aus den gemessenen Kraft-Abstands-Kurven (s. Kap. 3.2.1) extrahiert. Es ist zu erkennen, dass die Adhäsionskraft über die Tempe- 
a) kristallines $\mathrm{PdCuSi}$

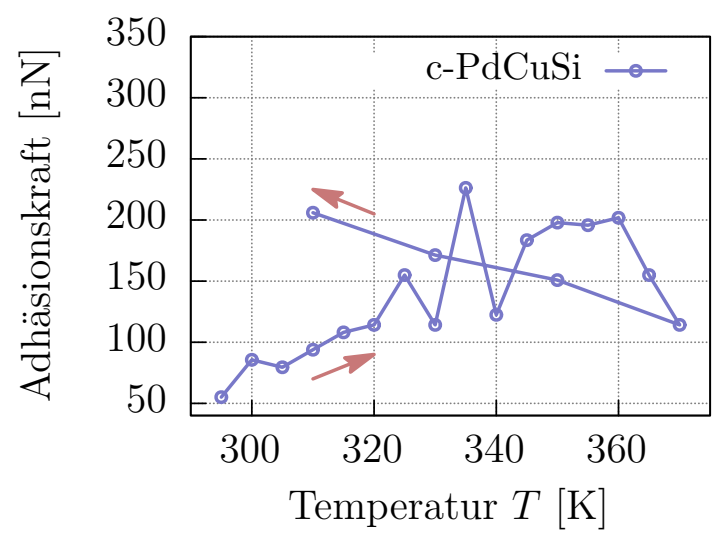

b) amorphes $\mathrm{PdCuSi}$

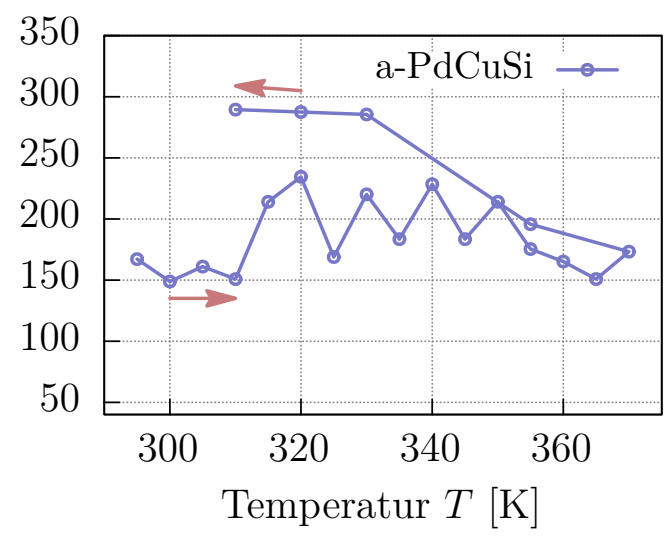

Abbildung 8.3 - Die Adhäsionskraft der a) kristallinen und der b) amorphen Probe ist aufgrund der großen Kontaktfläche relativ groß. Mit zunehmender Anzahl der Messungen wird sie größer. Bei hohen Temperaturen kann man eine Abnahme erkennen. Aufgrund des zeitlichen Verlaufs kann man die Änderung der Adhäsionskraft einer Änderung der Spitzengeometrie zuordnen. Auch hier ist keine Übereinstimmung mit der gemessenen Dämpfung zu erkennen.

ratur konstant im Bereich von $(67 \pm 8)$ nN liegt und daher als Ursache der gefunden Dämpfungsänderung ausgeschlossen werden kann.

Abbildung 8.3 zeigt die Adhäsionskraft der $\mathrm{Pd}_{77,5} \mathrm{Cu}_{6} \mathrm{Si}_{16,5}$-Proben. Zuerst ist die kristalline Probe vermessen worden. Man erkennt in a), dass die Adhäsionskraft mit der Temperatur zunimmt. In Kapitel 7 ist angedeutet worden, dass sich die Spitze während der Messung verändert haben muss, da zunächst Doppelpeaks gesehen worden sind. Das Abreiben der Spitze führt zur Vergrößerung der Kontaktfläche und damit zu höheren Adhäsionskräften. Oberhalb $360 \mathrm{~K}$ nimmt die Adhäsionskraft wieder ab, was als Verdampfen des Wasserfilms auf der Oberfläche verstanden werden kann. Im Kühlprozess nimmt die Adhäsionskraft weiter zu.

Mit identischer Spitze ist anschließend die amorphe Probe vermessen worden. Daher ist der Wert der Adhäsionskraft ähnlich zum letzten Wert auf der kristallinen Probe. Man erkennt in Abbildung $8.3 \mathrm{~b}$ ), dass die Adhäsionskraft im Heizprozess bis zu einer Temperatur von etwa $320 \mathrm{~K}$ sehr leicht ansteigt, anschließend sättigt und schließlich wie bei der kristallinen Probe bei hohen Temperaturen abnimmt. Auch im Kühlprozess steigt die Adhäsion wieder an.

Die Messungen sind im Kräftebereich von $960 \mathrm{nN} \leq F_{\mathrm{N}} \leq 48 \mu \mathrm{N}$ durchgeführt worden, so dass die Adhäsionskraft im Verhältnis zur von außen angelegten Kraft klein ist. 
Zusammenfassend kann man sagen, dass die gemessenen Änderungen der Adhäsionskräfte aufgrund der kontrollierten Luftfeuchtigkeit überwiegend einer Spitzenänderung zugeordnet werden können. Vor allem können die gefundenen Dämpfungsänderungen nicht mit der Adhäsion erklärt werden, da sie nicht dem gleichen Trend folgen.

\subsection{DÄMPfUNGSPROZESSE}

Im folgenden Abschnitt sollen gewonnene Messdaten mit Literaturdaten verglichen und mögliche Dämpfungsprozesse dargestellt werden. Da weder die Reibung noch die physikalischen Eigenschaften der Probensysteme vollständig verstanden sind, können diese Interpretationen nur phänomenologischer Natur anhand der gewonnenen Messdaten sein.

\subsubsection{PdCuSi}

Relaxationsprozesse eines amorphen Systems können analysiert werden, indem das Dämpfungsverhalten untersucht wird. Mit einem Lunkenheimer-Loidl-Plot (s. Abb. 8.4) wird das Relaxationsspektrum eines amorphen Systems schematisch dargestellt. Der sogenannte Boson-Peak ist Gitterschwingungen zuzuordnen [Chumakov04]. Er führt zum Verlust bei sehr hohen Frequenzen im Bereich von $10^{10}$ bis $10^{14} \mathrm{~Hz}$. Bei kleineren Frequenzen tritt der zweite Relaxationsprozess oder auch $\beta$-Prozess auf [Yu13, Yu14]. Nach Kapitel 4.2.1 entspricht er in der potentiellen Energielandschaft Übergängen von einem zum anderen lokalen Minimum. Die $\beta$-Relaxation nimmt je nach System unterschiedliche Formen an. Es sind Peaks, breitere Hügel oder flachere Flügel (Wing) beobachtet worden. Amorphes $\mathrm{Pd}_{77,5} \mathrm{Cu}_{6} \mathrm{Si}_{16,5}$ zeigt typischerweise die Form eines breiten Hügels [Yu13]. Der $\beta$-Prozess geht meistens in ein größeres Maximum über, das den $\alpha$-Prozess - und damit viskoses Fließen - beschreibt. Die Auftragung in Abbildung 8.4 ist stark temperaturabhängig. Vor allem der $\alpha$-Prozess, aber auch der $\beta$-Prozess verschieben in Richtung höherer Frequenzen bei steigender Temperatur [Lunkenheim00].

Die Messmethode zur Untersuchung von Relaxationsdynamiken ist üblicherweise die dielektrische Spektroskopie. Aufgrund des Zeit-Temperatur-Superpositionsprinzips, das für metallische Gläser bestätigt wurde [O'Connell97, Meyer99, Richert02], kann man aber auch mit konstanter Frequenz als Funktion der Temperatur verschiedene Relaxationen anregen. Durch eine Auftragung des Verlustes gegen die 
Abbildung 8.4-Schematische Darstellung des frequenzabhängigen Verlustspektrums eines Glases in Form eines Lunkenheimer-Loidl-Plot. Nach [Lunkenheim00, Yu14].

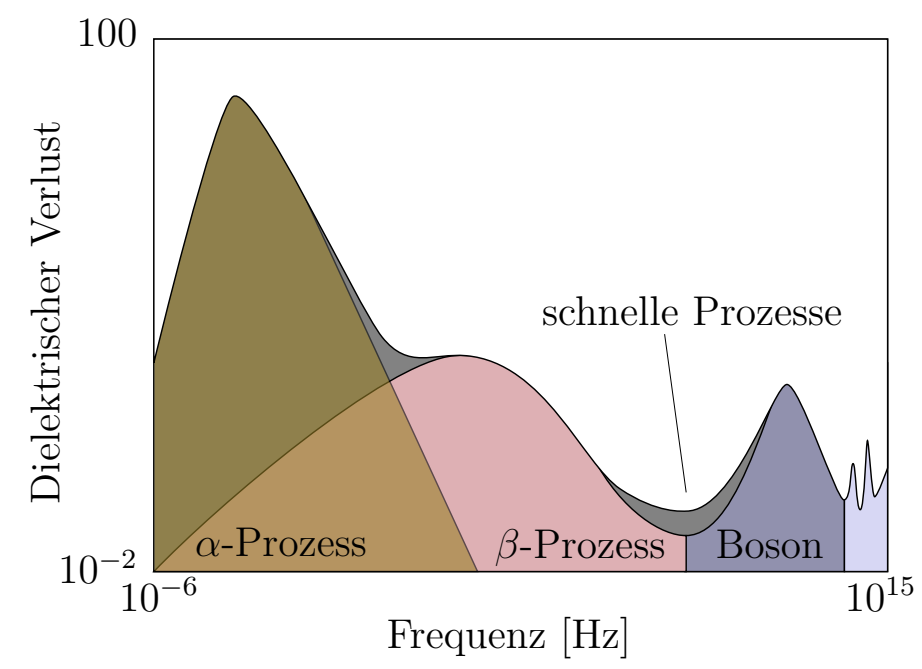

Temperatur wird die Lunkenheimer-Loidl-Auftragung an der $y$-Achse gespiegelt. Der Boson-Peak tritt dann bei tiefen Temperaturen auf. In Richtung hoher Temperaturen folgt zunächst die $\beta$-Relaxation und anschließend der $\alpha$-Peak.

Abbildung 8.5 zeigt den Verlustmodul von amorphem $\mathrm{Pd}_{77,5} \mathrm{Cu}_{6} \mathrm{Si}_{16,5}$ in Abhängigkeit von der Temperatur [Hachenberg06]. Hier wird deutlich, dass bei diesem Probensystem vor allem der $\alpha$-Prozess zum starken Ausschlag im Verlust führt. Dieser liegt jedoch bei $T \approx 670 \mathrm{~K}>T_{\mathrm{g}}$. Hinzu kommt, dass Bedorf et al. zeigen konnten, dass $\beta$-Relaxationen bei Probendicken $\leq 30 \mathrm{~nm}$ unterdrückt sind. [Bedorf10] Hier gilt, dass die Eindringtiefe der Messspitze nach dem Hertz'schen Modell $d=a_{c}^{2} / R$ und im Fall der höchsten Normalkraft $d \approx 12 \mathrm{~nm}$ ist. Die gefundene Dämpfung kann so also nicht der $\beta$-Relaxation zugeordnet werden.

Tabelle 8.1 zeigt, dass während der Messung hohe Drücke herrschen können. Es

Abbildung 8.5 - Schematische Darstellung und Messwerte des Verlustmoduls einer amorphen PdCuSi-Probe in Abhängigkeit von der Temperatur. Die Scangeschwindigkeit liegt hier bei $5 \mathrm{~K} \mathrm{~min}^{-1}$. Aus [Hachenberg06].

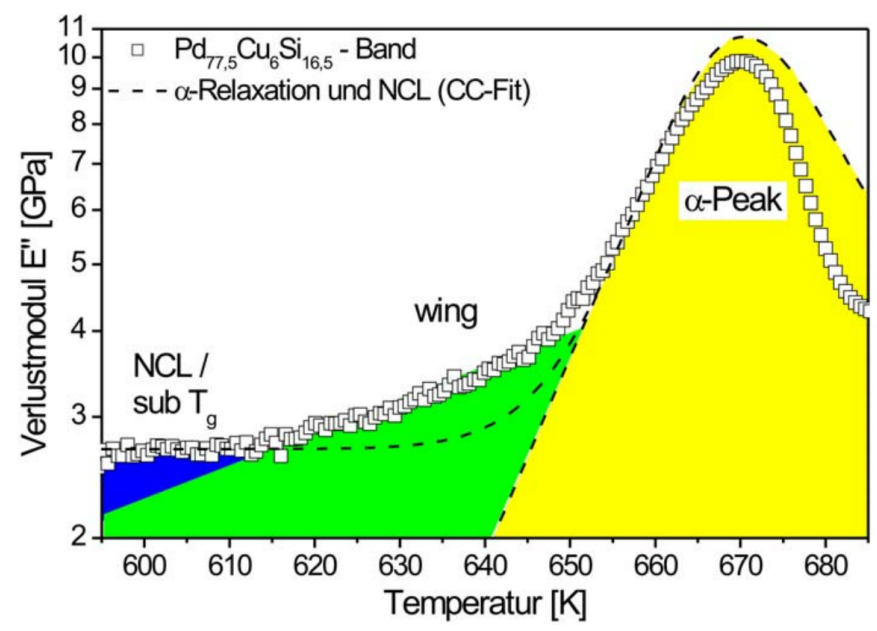


ist mittlerweile bekannt, dass für die $\alpha$-Relaxation eine mechanische Belastung eine ähnliche Rolle spielt wie die Temperatur selbst [Yu15]. Guan et al. [Guan10] zeigen anhand von Computersimulationen am System $\mathrm{Zr}_{50} \mathrm{Cu}_{40} \mathrm{Al}_{10}$, dass für Kurven konstanter Viskosität die Beziehung

$$
1=\frac{T}{T_{0}}+\left(\frac{\sigma}{\sigma_{0}}\right)^{2}
$$

gilt. Nimmt man an, dass für PdCuSi $\sigma_{0} \approx 1,5 \mathrm{GPa}$ [Golding72, Johnson05, Liu05, Wagner11b] gilt und dass $T_{0}=675 \mathrm{~K}$ die Temperatur des $\alpha$-Prozesses ist, muss bei Temperaturen $T=300 \mathrm{~K}$ eine Spannung von $\sigma \approx 1,13 \mathrm{GPa}$ herrschen, um die gleiche Viskosität für den $\alpha$-Prozess bei $\sigma=0 \mathrm{zu}$ erreichen.

$\mathrm{Yu}$ et al. finden, dass eine mechanische Belastung in metallischen Gläsern nicht nur Relaxationsdynamiken beschleunigt, Peaks also in Richtung tieferer Temperaturen verschiebt, sondern auch fragile Glasbildner zu stärkeren Glasbildnern macht. Das hat zur Folge, dass das Relaxationsspektrum der $\alpha$-Relaxation auf der Seite der tiefen Temperaturen mit steigender Belastung breiter wird, bei hohen Temperaturen hingegen gleich bleibt. [Yu15]

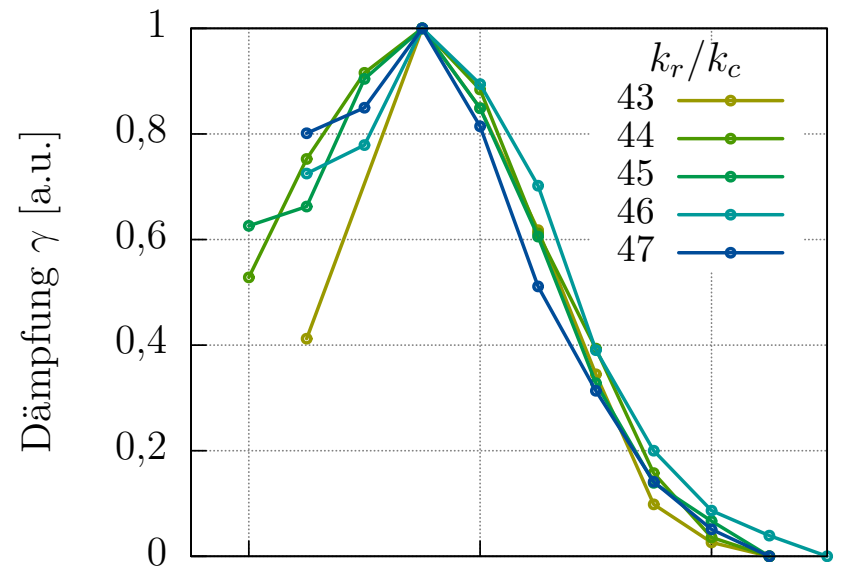

Abbildung 8.6 - Die Dämpfung $\gamma$ der a-PdCuSi-Probe. Zur Vergleichbarkeit sind Maxima übereinander geschoben und Messdaten normiert worden.

Temperatur $T$ [a.u.]

Abbildung 8.6 zeigt die gemessene Dämpfung der amorphen Probe aus Kapitel 7. Es sind hier nur die Werte von Raumtemperatur bis zum Minimum verwendet worden. Diese sind zunächst auf den Wertebereich 0 bis 1 normiert und anschließend so verschoben worden, dass die Maxima übereinanderliegen. Man kann erkennen, dass die Flanke bei höheren Temperaturen für alle Kontaktsteifigkeiten übereinanderliegt und auch, dass dies bei tieferen Temperaturen nicht der Fall ist. Ein Trend wie 
$\mathrm{Yu}$ et al. ihn beschreiben, ist aber nicht eindeutig zu erkennen, da der Messbereich durch das Gerät auf Messungen oberhalb der Raumtemperatur beschränkt ist.

Auch wenn die verwendeten Normalkräfte und die daraus resultierenden Drücke dazu in der Lage sind, den $\alpha$-Peak in den vermessenen Temperaturbereich zu verschieben, kann dies nicht abschließend bewiesen werden.

Weitere Anhaltspunkte, die für einen druckinduzierten $\alpha$-Peak sprechen, sind zum einen, dass der Peak bei höheren Drücken um etwa $10 \mathrm{~K}$ in Richtung tiefer Temperaturen verschiebt (vgl. Abb. 7.12), zum anderen, dass die kristalline Probe keinen derartigen Peak zeigt. Geht man davon aus, dass das Verhalten von $\alpha$ Relaxationen stammt, hieße das, dass das Probenmaterial unterhalb der Spitze viskos fließt und der elastische Modul, also der Realteil des Verlustes, klein wird.

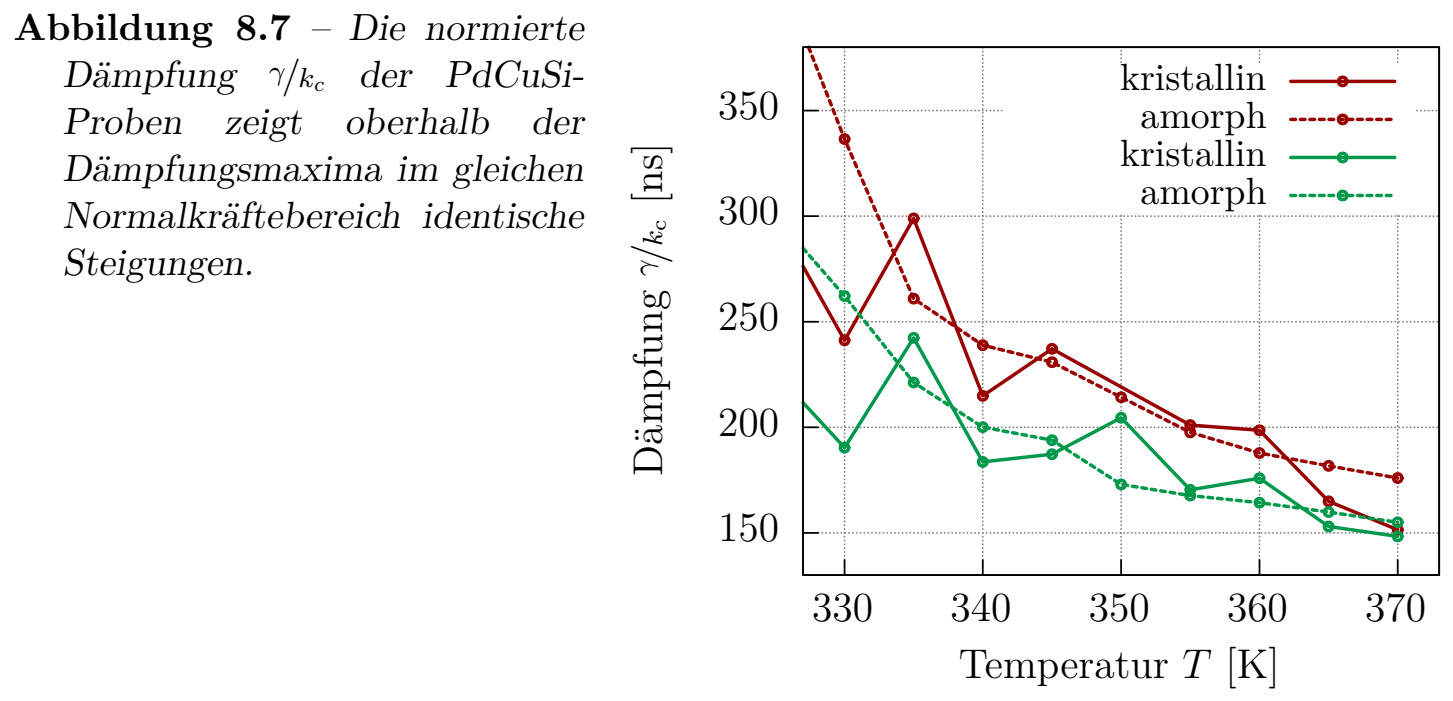

Abbildung 8.7 zeigt beispielhaft für beide PdCuSi-Proben jeweils zwei Messungen. Gleiche Farben entsprechen dem gleichen Normalkraftbereich. Man erkennt, dass die amorphe Probe (gestrichelte Linien) bei Temperaturen oberhalb des Dämpfungspeaks $(T \approx 335 \mathrm{~K})$ die gleichen Steigungen wie die kristalline Probe aufweist. Auch in Kapitel 6 ist bei hohen Drücken eine Erhöhung des Reibungskoeffizienten gefunden worden. Das deutet darauf hin, dass die Probe lokal kristallisiert und daher den höheren Reibungskoeffizienten der kristallinen Probe annimmt. Die erhöhte Normalkraft ist durch große externe Anregungen erreicht worden. Schaltet man die externe Anregung aus, wird immer noch der kristallisierte Teil der Probe vermessen, so dass verstanden werden kann, warum die Reibungskraft auch ohne externe Anregung größer geworden ist (vgl. Abb. 6.24). 


\subsubsection{LSMO}

Die Reibungsmessungen an $\mathrm{La}_{1-x} \mathrm{Sr}_{x} \mathrm{MnO}_{3}(x=0,4)$ in Abhängigkeit von der Temperatur zeigen mit beiden Messmethoden bis zu einer Temperatur von etwa $320 \mathrm{~K}$ eine Abnahme des Reibungskoeffizienten $\mu$ bzw. der Dämpfung $\gamma$ (s. Abb. 8.8). Bei höheren Normalkräften fällt die Reibung mit steigender Temperatur weiter ab (s. Abb. 8.8 a)). Verringert man jedoch die Normalkraft - und damit die Eindringtiefe der Spitze -, ändert sich das Verhalten (s. Abb. 8.8 b)). Bei fester Temperatur und gleicher Stelle auf der Probe wird die - mit der CR-FFM-Methode gemessene - Dämpfung größer, wenn die Normalkraft abnimmt. Auch in Abschnitt 6.8 ist mit einer detaillierteren Auswertung ein ähnliches Verhalten gefunden worden (vgl. Abb. 6.26 und 6.27). Abbildung 8.8 zeigt die Reibungswerte beider Messmethoden in Abhängigkeit von der Temperatur für hohe a) bzw. niedrige b) Normalkräfte. In rot sind dabei Messungen aus Kapitel 6, in blau aus Kapitel 7 dargestellt. Man kann erkennen, dass mit beiden Messmethoden qualitativ ähnliche Reibungen gewonnen worden sind. Bei hohen Auflagekräften fällt die Reibung mit der Temperatur ab. Bei niedrigen Normalkräften steigt die Dämpfung ab $T \approx 320 \mathrm{~K}$ wieder an.

a) hohe Normalkraft

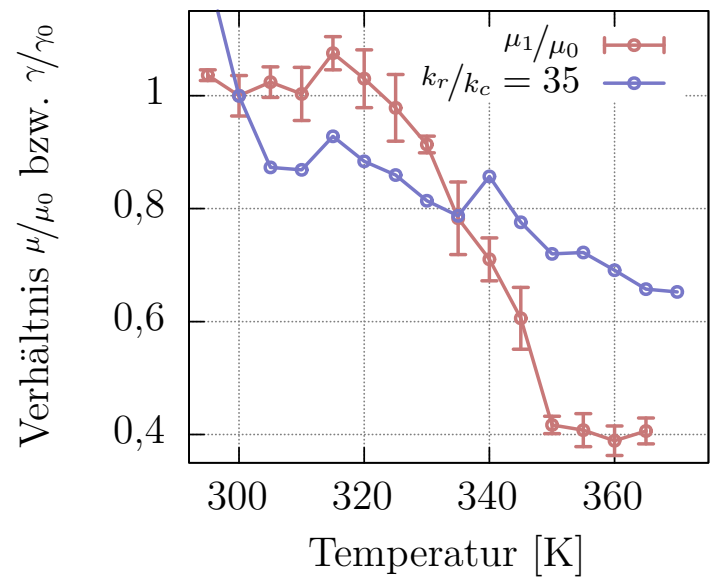

b) niedrige Normalkraft

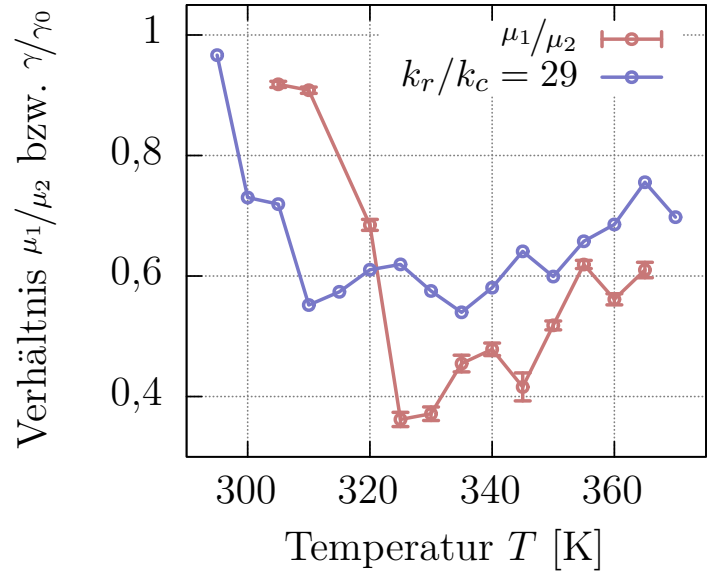

Abbildung 8.8 - Vergleich der Messdaten aus Kapitel 6 (rot) und Kapitel 7 (blau).

a) Bei hohen Normalkräften fällt die Reibung mit steigender Temperatur ab. b) Bei niedrigeren Drücken steigt die Reibung bei höheren Temperaturen wieder an. 


\section{THERMISCHE EFFEKTE}

In Abschnitt 2.3.1 ist der thermische Einfluss auf die Reibung beschrieben worden. Der unter dem Namen Thermolubrizität bekannte Effekt beschreibt, wie die Reibung mit steigender Temperatur abnimmt. In den Arbeiten, in denen Thermolubrizität gezeigt worden ist, wie zum Beispiel in [Jansen10, Riedo03], sind Normalkräfte, Blattfederkonstanten und Scangeschwindigkeiten verwendet worden, die alle mindestens eine Größenordnung unterhalb den hier verwendeten liegen. Trotzdem soll der thermische Effekt kurz diskutiert werden.

Die gemessene Dämpfung der $\mathrm{La}_{1-x} \mathrm{Sr}_{x} \mathrm{MnO}_{3}$-Probe hat bei allen Kontaktsteifigkeiten und Temperaturen bis $T \approx 315 \mathrm{~K}$ eine Abnahme gezeigt (vgl. Abb. 7.10). Geht man davon aus, dass unterhalb $T \leq 315 \mathrm{~K}$ die Dämpfung aufgrund thermischer Effekte abnimmt, kann mit Gleichung 2.11 eine Aktivierungsenergie $E_{\mathrm{a}}$ anhand einer Arrhenius-Auftragung bestimmt werden.

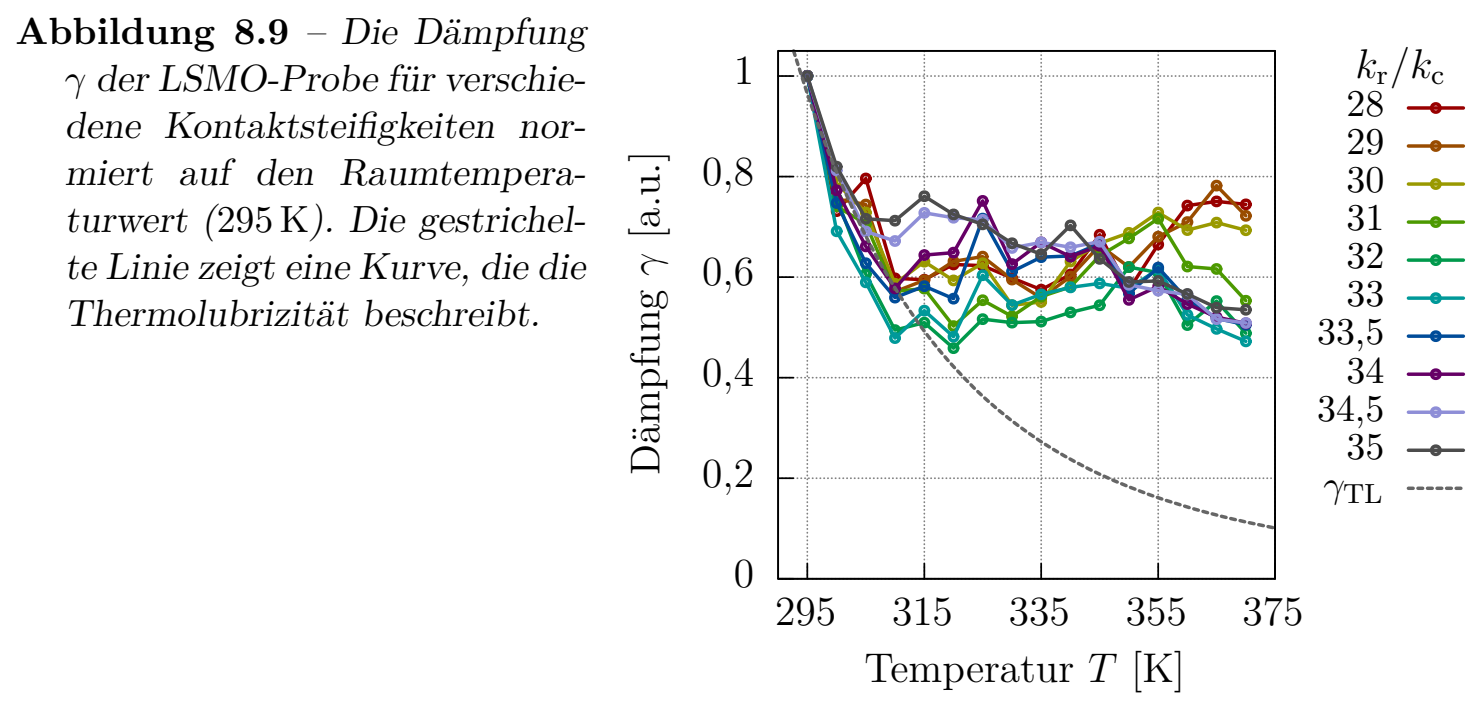

Abbildung 8.9 zeigt die - auf Raumtemperatur normierte - Dämpfung für verschiedene Kontaktsteifigkeiten. Die gestrichelte Linie zeigt hier beispielhaft eine Kurve der Funktion

$$
\gamma \propto \exp \left(\frac{E_{\mathrm{a}}}{k_{\mathrm{B}} T}\right)
$$

Zur Bestimmung der Aktivierungsenergie $E_{\mathrm{a}}$ ist für jeden Temperaturschritt $T \leq$ $315 \mathrm{~K}$ die durchschnittliche Dämpfung aller Kontaktsteifigkeiten bestimmt und anschließend mit einer Arrhenius-Auftragung eine lineare Anpassung durchgeführt worden. Der ermittelte Durchschnittswert $E_{\mathrm{a}}=267 \mathrm{meV}$ liegt in der Größenordnung der Werte von Kim et al. [Kim16] für Vanadiumdioxid $(0,3 \mathrm{eV})$ und von Barel et al. [Barel10a] für Silizium $(0,16-0,18 \mathrm{eV})$. 


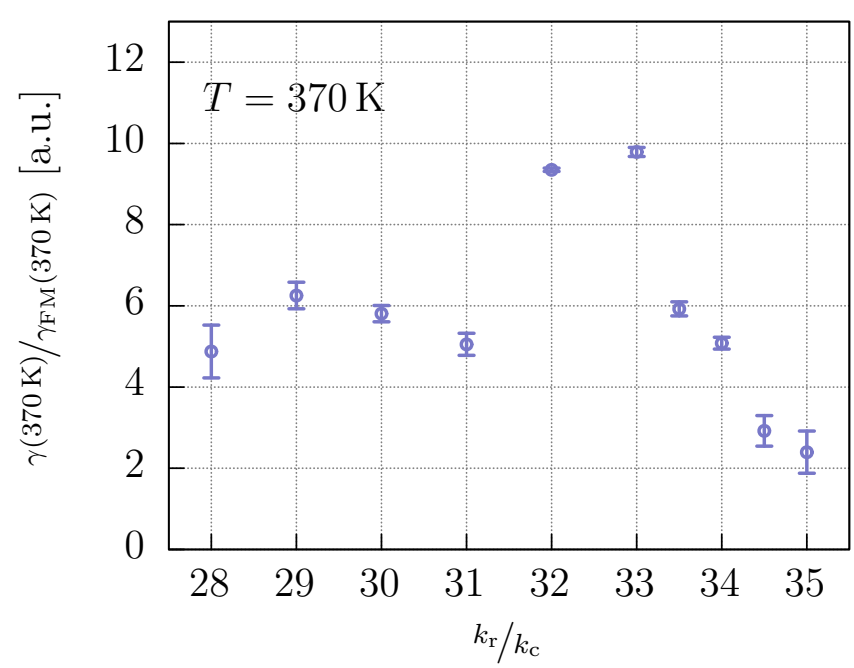

Abbildung 8.10 - Unter der Annahme, dass Thermolubrizität gültig ist, kann man das Verhältnis zwischen gemessenem Wert $\gamma(370 \mathrm{~K})$ und dem theoretischen Wert $\gamma_{T L}$ bei $T=370 \mathrm{~K}$ für verschiedene Kontaktsteifigkeiten $k_{r} / k_{c}$ bilden.

In Abbildung 8.9 ist zu erkennen, dass alle Dämpfungswerte bei Temperaturen $T \geq 315 \mathrm{~K}$ über der extrapolierten Kurve $\gamma_{\mathrm{TL}}$ liegen. Geht man davon aus, dass die Thermolubrizität für den gesamten Temperaturbereich gültig ist, kann bestimmt werden, um wie viel der gemessene Wert $\gamma$ vom theoretischen Wert $\gamma_{\mathrm{TL}}$ bei der Temperatur $T=370 \mathrm{~K}$ abweicht. Dazu ist für jede Kontaktsteifigkeit $k_{\mathrm{r}} / k_{\mathrm{c}}$ eine exponentiell abnehmende Kurve an die Messwerte bei tiefen Temperaturen gefittet und anschließend der Quotient $\gamma(370 \mathrm{~K}) / \gamma_{\mathrm{FM}}(370 \mathrm{~K})$ bestimmt worden. Abbildung 8.10 zeigt, dass die gemessenen Dämpfungen $\gamma$ bei der Temperatur $T=370 \mathrm{~K}$ mindestens um den Faktor 2,4 (bei $k_{\mathrm{r}} / k_{\mathrm{c}}=35$ ) und bis zu einem Faktor 9,8 (im Fall von $\left.k_{\mathrm{r}} / k_{\mathrm{c}}=33\right)$ größer sind als der theoretische Wert $\gamma_{\mathrm{FM}}$, den man bei Gültigkeit der Thermolubrizität erwarten würde. ${ }^{1}$

Die in Kapitel 6 mittels Sonolubrication bestimmten Reibungskoeffizienten der LSMO-Probe zeigen keinen Bereich mit logarithmischem Abfall der Reibung mit steigender Temperatur (vgl. Abb. 6.13). Es muss daher zunächst abgeschätzt werden, ob Thermolubrizität in den durchgeführten Messungen überhaupt eine Rolle spielen kann. Gleichung 2.10 in Abschnitt 2.3.1 zeigt, dass die Temperatur nur

\footnotetext{
${ }^{1}$ In Abschnitt 7.2.1 ist bereits erwähnt worden, dass die Dämpfung bei $k_{\mathrm{r}} / k_{\mathrm{c}}=35$ mit Ausnahme des Raumtemperaturwertes linear mit der Temperatur abfällt. In Abbildung 8.9 kann man erkennen, dass auch bei allen anderen Kontaktsteifigkeiten der Raumtemperaturwert (normiert auf 1) mindestens $20 \%$ größer ist als der Wert bei $300 \mathrm{~K}$. Es hat den Anschein, dass der Raumtemperaturwert aus anderen Gründen erhöht ist. Jedoch zeigen die Resonanzkurven bei $T=295 \mathrm{~K}$ die erwartete Lorentzform und auch die Kontaktresonanzfrequenzen liegen im gleichen Bereich wie die der höheren Temperaturen. Allerdings sind die gemessenen Amplituden und die reziproken Qualitätsfaktoren $Q^{-1}$ der Kontaktresonanzkurven bei $T=295 \mathrm{~K}$ etwa doppelt so groß wie im gesamten restlichen Temperaturbereich, wodurch sich eine höhere Dämpfung ergibt.
} 
deutlich unterhalb einer kritischen Scangeschwindigkeit

$$
v_{\mathrm{c}}=\frac{f_{0} k_{\mathrm{B}} T}{\sqrt{2} k a} \approx \frac{\sqrt{U_{0} / m a^{2}} k_{\mathrm{B}} T}{\sqrt{2} k} \approx \sqrt{\frac{U_{0}}{2 m}} \frac{k_{\mathrm{B}} T}{k a^{2}}
$$

großen Einfluss hat, da hohe Scangeschwindigkeiten die Wahrscheinlichkeit für thermisches Hüpfen verringern. Um die maximale kritische Geschwindigkeit $v_{\mathrm{c}}$ abzuschätzen, werden große Werte für Parameter im Zähler und kleine Werte für Parameter im Nenner gewählt. Nimmt man für die Temperatur $T=370 \mathrm{~K}$, das Potential $U_{0} \approx 0,5 \mathrm{eV}$ [Tshiprut09], die Masse $m=1 \cdot 10^{-12} \mathrm{~kg}$ [Barel10a], die Federkonstante $k=1 \mathrm{~N} \mathrm{~m}^{-1}$ und die Gitterkonstante $a=3 \AA$ an, ergibt sich die kritische Geschwindigkeit $v_{\mathrm{c}} \approx 11 \mathrm{\mu m} \mathrm{s}^{-1}$.

Die Frequenz der Blattfeder ist etwa $f \approx 730 \mathrm{kHz}$. Nimmt man an, dass die Spitze um nur $x=1 \AA$ ausgelenkt wird, ergibt sich für die laterale Geschwindigkeit im realen Experiment (CR-FFM) $v=f x \approx 73 \mu \mathrm{ms}^{-1}$. Daher ist Thermolubrizität hier zu vernachlässigen. ${ }^{2}$

\section{ELEKTRONISCHE REIBUNG}

In Abschnitt 2.3.2 sind elektronische Einflüsse auf die Reibung vorgestellt worden. Qi zusammen mit Park et al. [Park06, Qi08] haben in verschiedenen Arbeiten erhöhte Reibung an n-dotiertem Gallium-Arsenit und an p- und n-dotiertem Silizium gefunden (vgl. Abb. 2.12) und diese mit gefangenen Elektronen in einer Oxidschicht erklärt. Sie postulieren, dass die Spitze eine Spur von gefangenen Elektronen in einer Oxidschicht auf der Probe hinterlässt, die wiederum zu Bildladungen in der Spitze und damit zu einer elektrostatischen Anziehung führen.

In den Arbeiten von Qi bzw. Park et al. wird eine Überschussreibung

$$
\Gamma=\frac{F_{\mathrm{R}}}{v}
$$

(engl. Excess Friction) definiert, die die Reibungskraft $F_{\mathrm{R}}$ auf die Scangeschwindig-

\footnotetext{
${ }^{2}$ Warum die Kontaktresonanzamplituden bei $T=295 \mathrm{~K}$ größer sind als bei $T \geq 300 \mathrm{~K}$, kann nicht abschließend geklärt werden. Eine Ursache könnte die Spitze sein, die sich trotz vorherigem Abreiben bei $T=295 \mathrm{~K}$ und hohen Normalkräften noch einmal verändert hat. Da nach jeder Temperaturänderung aufgrund thermischer Ausdehnung eine andere Probenposition vermessen wird, könnte bei $T=295 \mathrm{~K}$ auch die Kontaktfläche aufgrund von Schmutz oder lokalen Rauigkeiten vergrößert sein, was zu höheren Amplituden führen würde. Dagegen spricht jedoch, dass die gemessene Adhäsionskraft keine Auffälligkeit bei $T=295 \mathrm{~K}$ zeigt (vgl. Abb. 8.2).
} 
keit $v$ normiert und so einen materialspezifischen Dämpfungsfaktor darstellt. Dieser Faktor kann also mit der gemessenen Dämpfung $\gamma$ verglichen werden. Quantitative Dämpfungsfaktoren aus Messungen, die in Kapitel 6 gezeigt worden sind, zu extrahieren, ist schwierig, da $\Gamma$ keine Normalkraftabhängigkeit enthält - die Reibungskraft $F_{\mathrm{R}}$ allerdings schon. Um die Messungen mit Literaturwerten vergleichen zu können, kann also nur die Größenordnung von $\Gamma$ verwendet werden.

Qi et al. finden in Galliumarsenid eine erhöhte Dämpfung $\Gamma \approx 5 \cdot 10^{-6} \mathrm{~N} \mathrm{~s} \mathrm{~m}^{-1}$ bei einer Normalkraft von $F_{\mathrm{N}}=3,5 \mathrm{nN}$ und Spannung von $1,5 \mathrm{~V}$ im Vergleich zur Spannung $-1,5 \mathrm{~V}$. Park et al. finden bei Spannung von $4 \mathrm{~V}$ eine erhöhte Reibung auf p-dotiertem Silizium $\Gamma \approx 2 \cdot 10^{-4} \mathrm{Ns} \mathrm{m}^{-1}$ im Vergleich zu n-dotiertem Silizium. Kim et al. [Kim16] haben Reibung auf Vanadiumdioxidschichten untersucht. Anhand der Messdaten (vgl. Abb. 2.14) kann man eine Dämpfungserhöhung von $\Gamma \approx 1,5 \cdot 10^{-2} \mathrm{~N} \mathrm{~s} \mathrm{~m}^{-1}$ zwischen isolierender und leitender Phase bei $T=380 \mathrm{~K}$ und $F_{\mathrm{N}} \approx 55 \mathrm{nN}$ abschätzen. In den gezeigten CR-FFM-Messungen werden Dämpfungen im Bereich von $1 \cdot 10^{-6} \mathrm{~N} \mathrm{~s} \mathrm{~m}^{-1}$ bis $1 \cdot 10^{-5} \mathrm{~N} \mathrm{~s} \mathrm{~m}^{-1}$ ermittelt.

Qi et al. schätzen zunächst Ohm'sche Verluste ab. Dazu nehmen sie an, dass die Energiedissipation jedes Elektrons $\Gamma_{\mathrm{e}}=F_{\mathrm{e}} / v=2 m_{\mathrm{e}} \tau^{-1}$ ist. Die Relaxationszeit $\tau$ schätzen sie mit dem Drude-Modell $\tau=m_{\mathrm{e}} / n e^{2} \rho$ ab, wobei $n$ die Elektronendichte, $m_{\mathrm{e}}$ die Elektronenmasse und $e$ die Elementarladung ist [Charles02]. Dazu ist der elektrische Widerstand $\rho$ gemessen worden. Sie berechnen $\Gamma_{\mathrm{e}} \approx 1,2 \cdot 10^{-18} \mathrm{~N} \mathrm{~s} \mathrm{~m}^{-1}$, was etwa 12 Größenordnungen unterhalb des gemessenen Wertes liegt. Auch mit typischen Stoßzeiten in Metallen von $\tau \approx 1 \cdot 10^{-14} \mathrm{~s}$ berechnet sich $\Gamma_{\mathrm{e}}=1 \cdot 10^{-16} \mathrm{~N} \mathrm{~s} \mathrm{~m}^{-1}$. Die Energiedissipation über Stöße der Elektronen ist daher zu klein, um das Verhalten der Reibung zu beschreiben. Außerdem führt die Annahme, dass Energiedissipation nur über Elektron-Elektron-Stöße stattfindet, dazu, dass die Reibung proportional zum Widerstand $\rho$ wäre. Denn die Elektronendichte ist über den Phasenübergang konstant [Dildar12, Westerburg00], so dass sich $\Gamma_{\mathrm{e}} \propto \tau^{-1} \propto \rho$ ergibt. Dass die Reibung proportional zum Widerstand ist, steht im Widerspruch zu den hier gezeigten Messungen und auch zu den Messungen von Kim et al. auf $\mathrm{VO}_{2}$. Denn Kim et al. finden in der metallischen Phase eine erhöhte Reibung im Vergleich zur isolierenden Phase und in dieser Arbeit konnte ein höherer Reibungskoeffizient in der ferromagnetischen Phase gefunden werden, die einen niedrigeren elektrischen Widerstand $\rho$ als die paramagnetische Phase hat.

Schließlich postulieren Qi et al. gefangene Ladungen in einer Oxiddeckschicht als Ursache für die erhöhte Reibung. Lebenszeiten von $\tau \approx 1 \mathrm{~ms}$ führen bei Scangeschwindigkeiten von $8 \mu \mathrm{m} \mathrm{s}^{-1}$ zu insgesamt 6 Bild- und gefangenen Ladungen. Qi 
et al. nehmen an, dass die Bildladungen etwa $1 \mathrm{~nm}$ von den gefangenen Ladungen entfernt sind, so dass die entstehende Kraft $F=0,03 \mathrm{nN}$ berechnet werden kann. Ähnlich argumentieren Kim et al. Sie finden mit XPS-Messungen eine $\mathrm{V}_{2} \mathrm{O}_{5}$ Schicht, die bis $540 \mathrm{~K}$ isolierend bleibt [Kang11, Kim16], in der die Möglichkeit von gefangenen Ladungen existiert.

Im untersuchten LSMO sind keine derartigen isolierenden Oxidschichten anderer Zusammensetzungen bekannt, die gefangene Ladungen zuließen. Calderón et al. haben theoretische Berechnungen am $\mathrm{La}_{1-x} A_{x} \mathrm{MnO}_{3}$ durchgeführt. Sie finden, dass aufgrund der Unterdrückung einer kubischen Symmetrie an der Oberfläche Elektronen vom Bulk in die Oberfläche transferiert werden und sich so $\mathrm{Mn}^{3+}$-Ionen an der Oberfläche bilden. Das führt dazu, dass der Doppelaustausch geschwächt wird und sich ein antiferromagnetischer Zustand an der Oberfläche einstellt [Calderón99]. Schmidt [Schmidt18] konnte allerdings anhand von Strom-Spannungs-Kennlinien auf $\mathrm{La}_{0.7} \mathrm{Sr}_{0.3} \mathrm{MnO}_{3}$ zeigen, dass eine isolierende Deckschicht nicht erwartet wird und dass elektrostatische Beiträge zur Reibung die Reibungsmessungen nicht erklären können.

Der elektrostatische Beitrag, den Qi et al. postulieren, basiert auf Ladungen, die bei ausreichend großen Geschwindigkeiten hinter der Spitze nahe der Oberfläche sitzen. Im CR-FFM-Modus wird die Spitze nicht über die Oberfläche gezogen. Hier sollte ein elektrostatischer Effekt schon durch den Messaufbau klein sein. Da beide Messmethoden ähnliche Ergebnisse liefern, wird auch hier ein elektrostatischer Beitrag vernachlässigt.

\section{Phononische ReIBUnG}

In Abschnitt 2.3.2 sind phononische Beiträge zur Reibung dargestellt worden. Dabei ist nach Cieplak et al. [Cieplak94] die Dämpfung bzw. die Reibung proportional zur Streurate der Phononen. Typische Streuraten bei LSMO sind in der Größenordnung von $\tau^{-1}=1 \cdot 10^{13} \mathrm{~s}^{-1}$ (Größenordnung 0,1 eV) [Ray03, Müller08]. Damit ergibt sich nach Gleichung 2.14 eine Dämpfung der Größenordnung $\Gamma \approx 1 \cdot 10^{-5}$, was der Größenordnung der gemessenen Dämpfung entspricht.

Anhand von Raman-Spektren können Schwingungsprozesse untersucht werden. Raman-Messungen an $\mathrm{La}_{1-x} \mathrm{Sr}_{x} \mathrm{MnO}_{3}$ mit $x=0,35$ zeigen im hier untersuchten Temperaturbereich keine Moden, die in Abhängigkeit von der Temperatur entstehen oder verschwinden [Björnsso00]. Allerdings beobachten Björnsen et al. eine Intensitätsabnahme eines breiten Peaks im Raman-Spektrum in der metallischen Phase im Bereich von $750 \mathrm{~cm}^{-1}$, also deutlich oberhalb des Energiebereichs von Phononen 
[Björnsso00]. Sie gehen davon aus, dass dieses Verhalten elektronischen Ursprungs ist. Podobedov et al. zeigen mit Raman-Messungen an $\mathrm{LaMnO}_{3}$, dass die Magnetisierung der Probe mit dem Raman-Signal korreliert [Podobedov98]. Sie erklären den gefunden Raman-Shift nahe der Übergangstemperatur $T_{\mathrm{N}}$ von antiferromagnetischer zu paramagnetischer Phase mit Spin-Gitter-Relaxationen. Lobad et al. untersuchen $\mathrm{La}_{1-x} \mathrm{Sr}_{x} \mathrm{MnO}_{3}$ mit $x=0,3$ mit optischer Pump-Probe-Spektroskopie und finden eine erhöhte Spin-Gitter-Relaxationskonstante $\tau_{\mathrm{SL}}$ nahe $T_{\mathrm{C}}$ [Lobad00].

a) elektrischer Widerstand

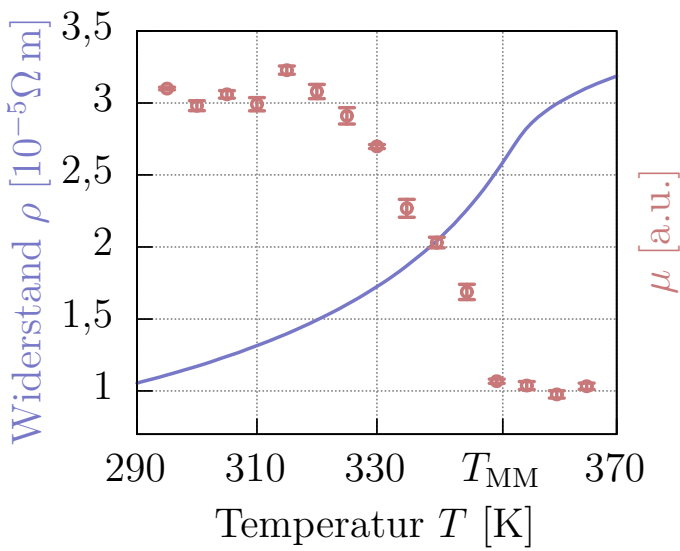

a) Magnetisierung

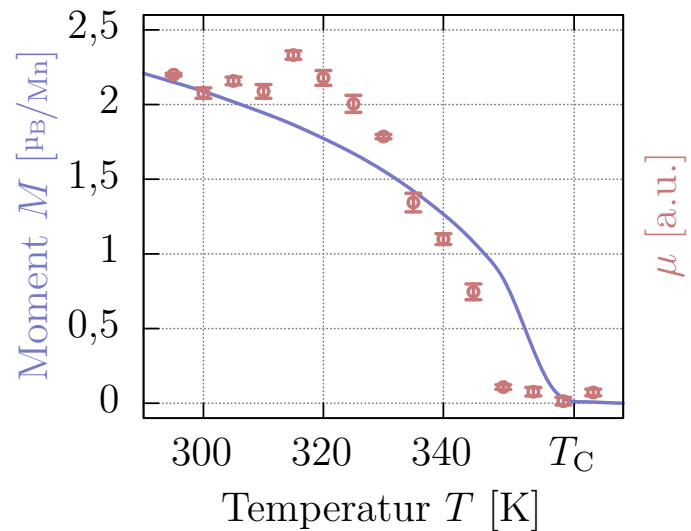

Abbildung 8.11 - Zum a) elektrischen Widerstand und b) zur Magnetisierung ist der gemessene Reibungskoeffizient $\mu$ in Abhängigkeit von der Temperatur aufgetragen. Auffällig ist, dass der Reibungskoeffizient etwa bei der Übergangstemperatur $T_{M M}$ sättigt.

Die Messspitze berührt die Probenoberfläche und koppelt so über das Gitter an das Elektronensystem. In der ferromagnetischen Phase ist die Spin-Spin-Wechselwirkung aufgrund der hohen magnetischen Ordnung erhöht. Dadurch ist die Elektron-Spin-Streurate $\tau_{\mathrm{e}-\mathrm{s}}^{-1}$ klein. In der paramagnetischen metallischen Phase ist der Doppelaustausch unterdrückt und dadurch $\tau_{\mathrm{e}-\mathrm{s}}^{-1}$ erhöht. Aufgrund der kleinen aber konstanten Elektron-Phonon-Kopplungskonstante $\lambda$ (s. Kap. 4.1.5) und der konstanten Elektronendichte $n$ [Westerburg00, Dildar12] ändert sich der elektrische Widerstand nach dem Drude-Modell $\rho=m_{\mathrm{e}} / n e^{2} \tau_{\mathrm{e}-\mathrm{s}}$ im Bereich $T_{\mathrm{C}}$ nur aufgrund der Änderung der Elektron-Spin-Wechselwirkung. Abbildung 8.11 zeigt den Reibungskoeffizienten, den Widerstand und die Magnetisierung in Abhängigkeit von der Temperatur. Dadurch dass im paramagnetischen Zustand die magnetische Ordnung reduziert ist, können mechanisch angeregte Elektronen schnell (fs) über 
die Elektron-Spin-Wechselwirkung relaxieren. Im ferromagnetischen Zustand ist die Relaxationszeit erhöht, da die ferromagnetische Ordnung eine Spinpolarisation fordert. Die hohe Spin-Spin-Wechselwirkung führt bei Temperaturen $T<T_{\mathrm{C}}$ dazu, dass der Elektron-Spin-Relaxationskanal (ps) [Müller08] nicht effektiv ist. Nach dieser Idee korreliert die Reibung des LSMO-Systems also antiproportional mit dem Widerstand, wie Abbildung 8.11 a) zeigt [Pfahl17].

Raman-Spektren können also zum Verständnis der Energiedissipation in diesem System beitragen. Das sollte prinzipiell auch mit anderen Systemen funktionieren. Die Reibungsmessungen von Kim et al. [Kim16] an Vanadiumdioxidschichten zeigen beispielsweise eine Korrelation mit Raman-Messungen von Goncharuk et al. [Goncharuk13]. Weit unterhalb der kritischen Temperatur $T_{\mathrm{C}}-$ also in der isolierenden Phase - nehmen mit steigender Temperatur die Intensitäten der charakteristischen Phononenlinien stark ab. Kurz unterhalb $T_{\mathrm{C}}$ entwickeln sich zwei Maxima, die mit steigender Temperatur zu einem breiten Maximum noch höherer Intensität zusammenwachsen, das mit stark-korrelierten Elektronen und großer Elektron-Phonon-Kopplung erklärt wird. Sehr große Elektron-Phonon-Kopplungen $(\lambda \approx 30-50)$ sind auch in [Okazaki04] mittels Photoemission gemessen worden.

Basierend auf den Raman-Spektren könnte man interpretieren, dass im isolierenden Zustand charakteristische Gitterschwingungen mit steigender Temperatur thermisch besetzt sind und so nicht mehr mechanisch angeregt werden können, so dass die Reibung abnimmt. Da die charakteristischen Phononenlinien (größte Intensität bei $f \approx 200 \mathrm{~cm}^{-1}$ ) bei niedrigeren Energien liegen als das breite entstehende Maximum (größte Intensität bei $f \approx 400 \mathrm{~cm}^{-1}$ ), ist die Dämpfung im metallischen Zustand deutlich erhöht. Mit Gleichung 2.13 kann man abschätzen

$$
\frac{\eta_{\mathrm{met}}}{\eta_{\mathrm{iso}}} \approx\left(\frac{200}{400}\right)^{4} \approx 16
$$

Wären allein die genannten Moden besetzt, ergäbe sich eine Dämpfungs- bzw. Reibungsänderung um den Faktor 16. Da aber in beiden Phasen auch andere RamanLinien niedrigerer Intensitäten auftreten, entspricht dieser Faktor der größten Abschätzung. Kim et al. messen eine Reibungserhöhung um den Faktor 11 [Kim16]. Andere Arbeiten zeigen Faktoren von 1,7 [Meer16] oder 1,5 [Sawatzky17].

Ein weiteres Indiz dafür, dass die Reibung mit dem Raman-Spektrum korreliert, ist, dass Kim et al. [Kim16] eine Reibungsänderung auf den vermeintlich metallischen Bereichen finden, die von der Normalkraft abhängt. Sie argumentieren, dass ein druckinduziertes Schalten in die metallische Phase stattfindet, so dass 
a) amorphes $\mathrm{PdCuSi}$

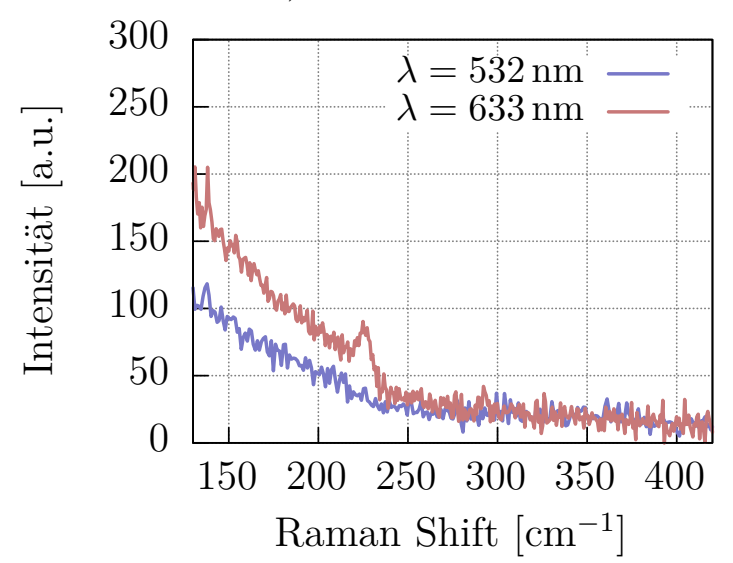

b) kristallines $\mathrm{PdCuSi}$

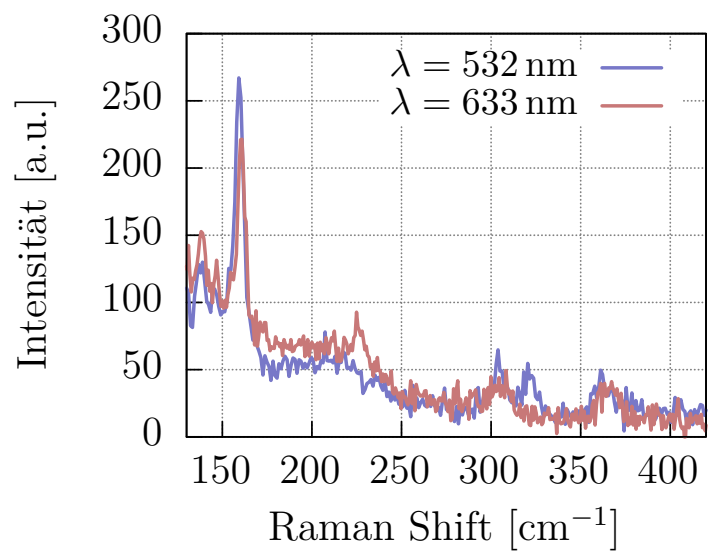

Abbildung 8.12 - Raman-Spektren des $\mathrm{Pd}_{77,5} \mathrm{Cu}_{6} \mathrm{Si}_{16,5}$-Systems in der a) amorphen und b) kristallinen Form. Die Spektren sind mit einem roten $(\lambda=633 \mathrm{~nm})$ und grünen Laser $(\lambda=532 \mathrm{~nm})$ aufgenommen worden. Messung von Sebastian Merten am I. Physikalischen Institut.

das System kurz vor $T_{\mathrm{C}}$ steht (s. Abb. 2.14). Das wiederum bedeutet, dass bei niedrigeren Drücken die isolierende Phase noch stabil ist. Aber auch hier ist schon eine Reibungserhöhung im Vergleich zur Phase, die über den gesamten vermessen Normaldruckbereich isolierend bleibt, zu erkennen (vgl. bspw. Messdaten der isolierenden und metallischen Phase bei $T=80^{\circ} \mathrm{C}$ in Abb. 2.14 a)). Kim et al. ignorieren diesen Bereich und bestimmen den Reibungskoeffizienten nur aus dem höheren Druckbereich. Goncharuk et al. [Goncharuk13] zeigen, dass der breite Peak im Raman-Signal schon etwa $20 \mathrm{~K}$ unterhalb $T_{\mathrm{C}} \mathrm{zu}$ erkennen ist, so dass auch die Reibungserhöhung vor $T_{\mathrm{C}}$ verstanden werden kann.

In Kapitel 6 ist gezeigt worden, dass amorphes $\mathrm{Pd}_{77,5} \mathrm{Cu}_{6} \mathrm{Si}_{16,5}$ einen kleineren Reibungskoeffizienten $\mu$ hat als die kristalline Variante. Auch hier sind Peaks im Raman-Spektrum zu erkennen (s. Abb. 8.12). In der kristallinen Phase kann Energie über Phononen ins System dissipiert werden. In der amorphen Phase existieren diese Moden nicht, der Dissipationskanal ist geschlossen und der Reibungskoeffizient ist kleiner als der der kristallinen Phase.

Sowohl in Kapitel 6 als auch in Kapitel 7 ist festgestellt worden, dass die Reibung bei sehr geringen Auflagekräften mit steigender Temperatur ab $T \approx 330 \mathrm{~K}$ wieder zunimmt (vgl. Abb. 6.26 a) und Abb. 7.10). Abbildung 6.13 zeigt, dass bei Temperaturen $T \leq 310 \mathrm{~K}$ eine Blattfederamplitude von $A_{\mathrm{C}} \geq 0,25 \mathrm{~nm}$ zu einer Abweichung 
von der erwarteten Normalparabel führt. Mit der analytischen Beschreibung (Gl. 6.6) kann die zugehörige Normalkraft $F_{\mathrm{N}} \approx 28 \mathrm{nN}$ bestimmt und damit der Kontaktradius $a_{\mathrm{c}} \approx 3,1 \mathrm{~nm}$ mit Gleichung 7.2 abgeschätzt werden. Da das Spannungsfeld in der Probe inhomogen ist, ist es schwierig, eine Tiefenwirkung abzuschätzen. Jedoch ist die maximale Schubspannung $p_{\max }$ bei einer Tiefe von etwa $d_{\max } \approx 0,48 a_{\mathrm{c}}$ erreicht [Johnson85], was hier $d_{\max } \approx 15 \AA$ ergibt. Bei hohen Temperaturen wird dieses Verhalten bei geringeren Blattfederamplituden - was höheren Normalkräften entspricht - beobachtet. Bei $T=360 \mathrm{~K}$ berechnet sich beispielsweise $d_{\text {max }} \approx 18,6 \AA$ (vgl. Abb. 6.28).

Im CR-AFM-Modus ist ein ähnliches Verhalten ab $k_{\mathrm{r}} / k_{\mathrm{c}}=30$, was einer Normalkraft von $F_{\mathrm{N}}=1,4 \mu \mathrm{N}$ entspricht, gefunden worden. Mit der gemessenen Spitzenform ergibt sich im Hertz'schen Kontakt eine maximale Schubspannung bei einer Tiefe von $\approx 6,9 \mathrm{~nm}$. Bei den kleinsten Auflagekräften, also bei $k_{\mathrm{r}} / k_{\mathrm{c}}=28$ zeigt die Dämpfung die größte Erhöhung bei hohen Temperaturen. Hier ist $p_{\max }$ bei einer Tiefe von etwa $d_{\max } \approx 4,8 \mathrm{~nm}$ erreicht. Für eine zylinderförmige Spitze sind diese Werte deutlich kleiner, so dass beide Messmethoden eine Änderung der Reibung in den obersten Lagen zeigen. Die Dicke dieser Schicht liegt bei Raumtemperatur bei einigen Einheitszellen.

Theoretische Berechnungen deuten darauf hin, dass die Oberfläche von Manganaten ein anderes Verhalten zeigt als das Bulk-Material [Quijada98, Calderón99, Filippetti00, Fang01]. LSMO beispielsweise entwickelt über Elektronendotierung einen Antiferromagneten an der Oberfläche. Calderón et al. berechnen die Orbitalbesetzung von $\mathrm{La}_{1-x} \mathrm{Sr}_{x} \mathrm{MnO}_{3}(x=0,3)$. Dabei führt das Fehlen der kubischen Struktur in Kombination mit dem Doppelaustausch dazu, dass Elektronen aus dem Bulk in die Oberfläche bewegt werden [Calderón99]. Dadurch bilden sich $\mathrm{Mn}^{3+}$ Ionen an der Oberfläche und es sind dort nur noch $\mathrm{d}_{3 z^{2}-r^{2}}$-Orbitale besetzt. An der Oberfläche wird dabei der Doppelaustausch unterdrückt, so dass sich nach Kapitel 4.1 ein Antiferromagnet einstellt. Die Berechnungen zeigen, dass nur die ersten drei Monolagen vom Bulk abweichen. Durch die Elektronendotierung stellt sich ein System ein, das chemisch dem von LSMO gleicht. Elektronisch ähnelt es eher einer geringeren Strontiumdotierung.

Mit der Surface-Enhanced-Raman-Spektroskopie (SERS) können Raman-Spektren von der Oberfläche aufgenommen werden. Abbildung 8.13 zeigt SERS-Messungen einer $\mathrm{La}_{1-x} \mathrm{Sr}_{x} \mathrm{MnO}_{3}(x=0,3)$-Probe auf einem $\mathrm{LaAlO}_{3}$-Substrat in Abhängigkeit von der Temperatur. Das Raman-Spektrum zeigt zum einen orthorhombische Phononenpeaks und zum anderen Jahn-Teller-Moden. LSMO ist weder orthor- 

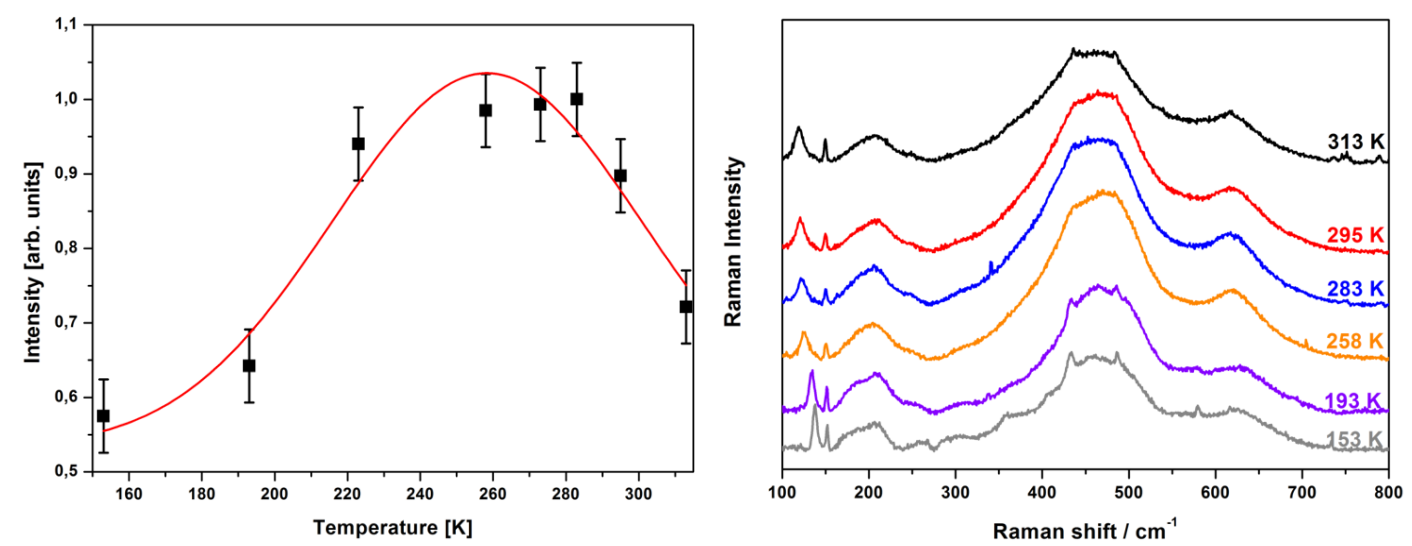

Abbildung 8.13 - Surface-Enhanced-Raman-Spektroskopie einer $\mathrm{La}_{1-x} \mathrm{Sr}_{x} \mathrm{MnO}_{3}(x=$ 0,3)-Probe auf $\mathrm{LaAlO}_{3}$-Substrat in Abhängigkeit von der Temperatur. Das RamanSpektrum zeigt zum einen orthorhombische Phononenpeaks und zum anderen JahnTeller-Moden, die beide im LSMO-Bulk nicht vorkommen. (Daten von Sebastian Merten am I. Physikalischen Institut.)

hombisch, noch zeigt es Jahn-Teller Verhalten. Die Intensität der Jahn-Teller-Mode in Abbildung 8.13 a) zeigt ein Maximum, das in LPCMO und LCMO typischerweise nahe der Übergangstemperatur gefunden wird [Moshnyaga14]. Auch wenn die Physik der Deckschicht von den Gitterparametern des Substrats abhängt und bei dem hier verwendeten STO ein anderer Typ von Antiferromagnet gefunden wird [Shibata18], kann die Intensitätkurve der Phononenpeaks in Abbildung 8.13 nur den Abfall der Reibung von tiefen Temperaturen bis zu $T=320 \mathrm{~K}$ erklären. SERS-Messungen von Dünnfilmen auf STO-Substraten sind nicht möglich, da die Phonenpeaks des Substrats die Peaks des Films überlagern.

Die Ergebnisse in Kapitel 6 und 7 deuten darauf hin, dass die Dicke der Deckschicht mit zunehmender Temperatur größer wird. Mit XRMS-Messungen ${ }^{3}$ konnten Verna et al. [Verna10] experimentell zeigen, dass eine magnetische „Totlage" auf $\mathrm{La}_{1-x} \mathrm{Sr}_{x} \mathrm{MnO}_{3}(x=0,33)$ existiert und dass diese mit der Temperatur dicker wird. Die Dicke ist bei $T=200 \mathrm{~K}$ etwa $17 \AA$ und wird $T=300 \mathrm{~K}$ etwa $21 \AA$, was in der gleichen Größenordnung wie das hier bestimmte $d_{\max }$ ist. Die Autoren messen keine Magnetisierung in der Deckschicht und erachten ein antiferromagnetisches Bild wie von Calderón berechnet - als sinnvolle Deutung.

Es existieren keine eindeutigen Ideen, die die Physik einer Manganatoberfläche beschreiben. Hinzu kommt, dass unterschiedliche Probenpräparationsmethoden unterschiedliche Sauerstoffleerstellen verursachen, die großen Einfluss auf die Physik

${ }^{3}$ engl. X-ray magnetic scattering 
der Manganate haben. Ein Vergleich von Literaturdaten untereinander ist daher schwierig. Die Literatur sowie die hier gezeigten Messungen deuten aber darauf hin, dass die Oberfläche einen anderen Effekt auf die Reibung hat als das BulkMaterial. In der Literatur ist die grundlegende Idee der Oberfläche meistens, dass die obersten Lagen ein Verhalten zeigen, als wären sie out-of-plane verzerrt. Unter statischem Druck verschwindet diese Verzerrung, so dass magnetische Totlagen unterdrückt werden und so der gemessene Reibungsanstieg wieder unterdrückt ist.

Da die Eigenschaften der Oberfläche nicht vollständig verstanden sind und teils kontrovers in der aktuellen Literatur zu Manganaten diskutiert wird, ist es schwierig, die gefundene Reibungserhöhung bei hohen Temperaturen zu erklären. Es könnte - ähnlich wie im ferromagnetischen Zustand - eine hohe Spin-Spin-Wechselwirkung der antiferromagnetischen Deckschicht dazu führen, dass angeregte Elektronen über Elektron-Phonon-Wechselwirkung relaxieren und so die Reibung erhöhen. Ebenso könnte eine Energiedissipation über Anregung der Jahn-Teller-Verzerrungen stattfinden, die mit SERS-Messungen gezeigt worden sind. Da die Deckschicht mit steigender Temperatur dicker wird, wird auch der vermessene Volumenanteil der Deckschicht größer, so dass die Erhöhung der Reibung bei höheren Temperaturen eventuell vom vermessenen Volumenverhältnis stammt.

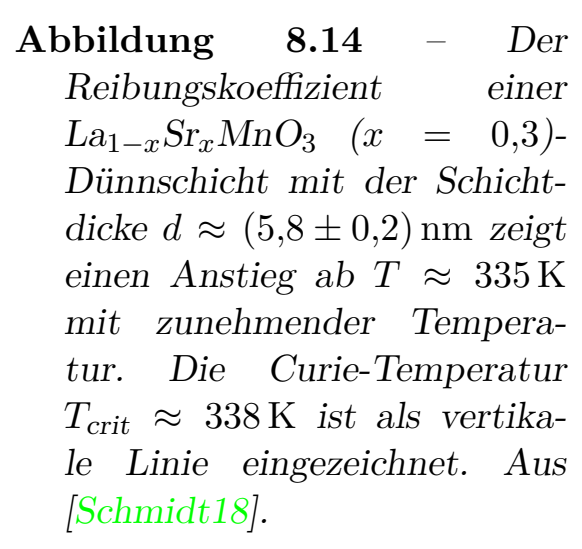

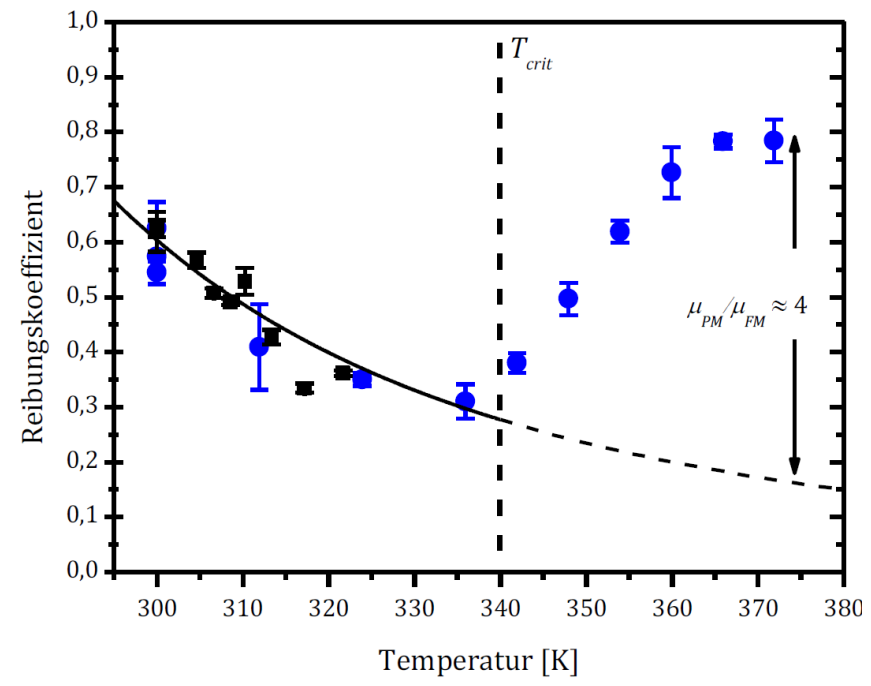

Für die Idee, dass die Oberfläche ein anderes Verhalten zeigt, spricht auch die Arbeit von Schmidt. Er findet einen Reibungsanstieg an einer $\mathrm{La}_{1-x} \mathrm{Sr}_{x} \mathrm{MnO}_{3}$ ( $x=0,3)$-Probe ab $T \approx 335 \mathrm{~K}$ bei einer maximalen Normalkraft von $25 \mathrm{nN}$ (s. Abb. 8.14) [Schmidt18]. In dieser Arbeit wird ein Schichtpaket untersucht, das aus einer $\mathrm{La}_{1-x} \mathrm{Sr}_{x} \mathrm{MnO}_{3}(x=0,3)$-Schicht auf einer $\mathrm{La}_{1-x} \mathrm{Sr}_{x} \mathrm{MnO}_{3}(x=0,6)$ - 
Schicht auf einem $\mathrm{SrTiO}_{3}$-Substrat besteht. Die Zwischenschicht wird dabei zur Entzerrung verwendet. Die untersuchte Deckschicht weist eine Schichtdicke von nur $d \approx(5,8 \pm 0,2)$ nm auf. Die Schichtdicke ist also nur etwa doppelt so groß wie die Dicke der magnetischen Totlagen, die Verna et al. [Verna10] bei $T=300 \mathrm{~K}$ gemessen haben. Die Vermutung liegt nahe, dass in der Arbeit von Schmidt [Schmidt18] vor allem die Deckschicht vermessen wurde. 


\section{Zusammenfassung Und AUSBLICK}

\section{ZUSAMMENFASSUNG}

In der vorliegenden Dissertation ist Reibung ausgewählter Materialien studiert worden. Dabei wurde ein kommerzielles Rasterkraftmikroskop unter kontrollierter Atmosphäre verwendet. Zusätzlich wurden Schallwandler eingesetzt, um die Blattfeder periodisch mit ihrer Eigenfrequenz anzuregen.

In Kapitel 6 mit dem Titel Sonolubrication ist das Verhalten dieser Anregung genauer studiert und analysiert worden. Es konnte gezeigt werden, dass die Normalkraft, die die Spitze ausübt, universell mit der Amplitude der Blattfeder abnimmt. Da der Reibungskoeffizient - wie im theoretischen Teil gezeigt - stark von der Normalkraft abhängt, kann mit Variation der Blattfederamplitude Reibung untersucht werden. Basierend auf einem semi-empirischen Kontaktpotential aus der Literatur ist in Kapitel 6 eine genaue analytische Beschreibung des Verhaltens der Normalkraft entwickelt worden. Darauf basierend ist ein neuer Parameter $\Delta F$ verwendet worden, der die Unterschiede der Reibungskraft mit und ohne Anregung beschreibt. Dazu sind klassische Reibungshysteresen im FFM-Modus aufgenommen worden. Nach jeder Hysterese ist eine weitere mit Anregung aufgenommen worden. Es hat sich gezeigt, dass sich der Parameter hervorragend dazu eignet, Reibung zu studieren, da er aufbaubedingte Messfehler reduziert und proportional zum Reibungskoeffizienten ist.

Unter dem Titel CR-FFM ist in Kapitel 7 eine neuartige Messmethode verwendet worden, um die Reibung zu studieren. Dabei wird die Messspitze im Kontakt wieder extern angeregt, ihre Position aber nicht verändert, wie es beispielsweise im FFM-Modus der Fall ist. Die zugrunde liegende Idee ist dabei, dass sich aufgrund der externen Anregung ein Teil der Spitze auf der Oberfläche bewegt. Ein Teil der gemessenen Dämpfung kommt also von diesem sogenannten Micro-Slip-Verhalten. Löst sich die Spitze komplett von der Oberfläche der Probe, führt das dazu, dass die Kontaktresonanzkurven die typische Lorentzform verlieren. Stellt man die An- 
regung so ein, dass die Lorentzform gerade noch nicht verloren wird, so entsteht eine hoher Anteil der Dämpfung durch Reibung, so dass mit dieser Methode ebenfalls Reibung studiert werden kann. Vorteilhaft ist hier, dass die Messung noch lokaler ist, als klassische Reibungshysteresen.

Um die Physik der Reibung zu untersuchen, sind Materialien verwendet worden, die sich durch zwei unterschiedliche Zustände auszeichnen. Das ist sinnvoll, da so gefundene Reibungsänderungen dem Unterschied der Zustände zugeordnet werden können. Auf der einen Seite ist das System $\mathrm{Pd}_{77,5} \mathrm{Cu}_{6} \mathrm{Si}_{16,5}$ ausgewählt worden, da es in amorpher und in kristalliner Form vorliegen kann. Auf der anderen Seite ist ein Übergangsmetalloxid des Systems $\mathrm{La}_{1-x} \mathrm{Sr}_{x} \mathrm{MnO}_{3}$ mit der Dotierung $x=0,4$ ausgesucht worden, da es einen Phasenübergang bei der Temperatur $T_{\mathrm{mm}} \approx 350 \mathrm{~K}$ zeigt, die im verwendeten Messaufbau mit einer Heizstation erreicht werden kann. Das System ist bekannt dafür, dass es von einer ferromagnetischen metallischen Phase bei tiefen Temperaturen zu einer paramagnetischen metallischen Phase bei hohen Temperaturen wechselt. Der temperaturgetriebene Phasenübergang dieses Systems ist insofern interessant, als sich weder Struktur noch Vibrationsmoden noch Elektron-Phonon-Kopplungskonstante noch Elektronendichte ändern. Einzig die Elektron-Spin-Wechselwirkung verändert sich, da sich die magnetischen Eigenschaften ändern.

Mit diesem Wissen konnte die Reibungsminderung der LSMO-Probe dem unterdrückten Dissipationskanal zugeordnet werden. Aufgrund der Magnetisierung muss in der ferromagnetischen Phase eine Energiedissipation über die Elektron-PhononWechselwirkung stattfinden. Die Reibung ist somit in der ferromagnetischen Phase erhöht. Außerdem ist ein Oberflächeneffekt gefunden worden, der vermutlich aufgrund einer antiferromagnetischen Deckschicht entsteht. Eine derartige Deckschicht ist anhand von theoretischen Berechnungen vermutet und auch experimentell vermessen worden. Die Untersuchung einer derartigen Deckschicht ist aktueller Stand der Forschung, daher ist eine genaue Zuordnung der gefundenen Reibungserhöhung schwierig. Jahn-Teller-Polaronen und die antiferromagnetischen Eigenschaften kommen als Ursache in Frage.

Die amorphe Probe zeigte im Vergleich zum kristallinen Gegenstück ein Maximum in der Dämpfung. Es konnten einige Argumente vorgebracht werden, die dafür sprechen, dass es sich um den $\alpha$-Prozess handelt, so dass die Probe lokal kristallisiert. Dass sich die Dämpfung und der Reibungskoeffizient der Probe oberhalb der Temperatur des $\alpha$-Prozesses wie die der kristallinen Probe verhalten, spricht stark dafür, dass es sich lokal um kristallines Material handelt. 
Mit diesen Ergebnissen konnte gezeigt werden, dass die Normalkraft in Kombination mit der Spitzengeometrie eine große Rolle spielt, da man bei kleinen Kontaktflächen schnell Drücke erreicht, die die Eigenschaften des Systems verändern. Zum einen konnte gezeigt werden, dass der Druck ausreicht, um den $\alpha$-Peak des amorphen $\mathrm{Pd}_{77,5} \mathrm{Cu}_{6} \mathrm{Si}_{16,5}$ um etwa $350 \mathrm{~K}$ zu verschieben. Zum anderen konnte der Einfluss der Deckschicht der LSMO Probe unterdrückt werden. Es sind zwei Möglichkeiten dafür genannt worden. Theoretische Berechnungen zeigten, dass eine out-of-plane-Ausdehnung Ursache der Deckschicht ist, so dass die Drücke ausreichen, um die antiferromagnetische Schicht zu unterdrücken. Es könnte aber auch sein, dass die Eindringtiefe der Spitze und damit der vermessene Volumenanteil der Deckschicht entscheidend ist.

\section{AusBLiCK}

Die CR-FFM-Methode produziert Ergebnisse, die qualitativ gut mit denen der FFM-Methode übereinstimmen. Allerdings gehen in die gemessene Dämpfung $\gamma$ auch Beiträge der internen Reibung der Probe und der Dämpfung der Luft ein. Die einzelnen Beiträge der Dämpfung zu separieren, ist bisher noch nicht möglich. Daher muss der Übergang vom starren Verhalten ohne Anregung bis zum vollständigen Loslösen der Spitze mit Anregung weiter untersucht werden. Weitere Arbeiten zu diesem Verhalten stehen bereits vor der Veröffentlichung. Ma et al. [Ma18] konnten unter anderem zeigen, dass bei Materialien mit kleiner innerer Reibung hohe Normalkräfte dazu führen, dass die Dämpfung der Blattfeder überwiegend von der Luft kommt und so nicht mehr vernachlässigbar bleibt.

Die Dämpfungsänderung der amorphen Probe in Abhängigkeit von der Temperatur ist mit dem $\alpha$-Prozess und lokalem Kristallisieren interpretiert worden. Die Korrektheit dieser Interpretation muss weiter überprüft werden. Zum einen könnten weitere amorphe Proben mit unterschiedlichen Glasübergangstemperaturen verwendet werden. Bei gleicher Normalkraft müssten die Dämpfungspeaks der Proben die gleiche Temperaturabhängigkeit zeigen wie die der Glasübergangstemperaturen, die man beispielsweise mit der DSC misst. Zum anderen muss die Normalkraftabhängigkeit genauer studiert werden. Je größer die Normalkraft, desto tiefer ist die Temperatur des Dämpfungspeaks. Hierbei könnten unterschiedliche Blattfedern helfen, um einen deutlich größeren Kräftebereich abzudecken.

Die Änderung der magnetischen Eigenschaften am Übergang der LSMO-Probe kann die gefundenen Reibungsmessungen erklären. Um diese Interpretation weiter zu verifizieren, wäre es sinnvoll, die Reibung zusätzlich in externen Magnetfeldern 
unterschiedlicher Stärke zu untersuchen. Interessant wäre beispielsweise die Reibung an Systemen zu untersuchen, bei denen der kolossale Magnetowiderstand stark ausgeprägt ist. Bei konstanter Temperatur könnte so in Abhängigkeit des externen Magnetfelds untersucht werden, ob die Reibung proportional zum Widerstand ist.

In dieser Dissertation wurde gezeigt, dass sich die Oberfläche der LSMO-Probe anders verhält als das Bulk-Material. Tip-Enhanced- oder Surface-Enhanced-Ramanmessungen (TERS und SERS) könnten zum Verständnis dieser Deckschicht beitragen. Über schichtdickenabhängige TERS- oder SERS-Messungen auf unterschiedlichen Substraten könnte die Verzerrung des LSMO-Films verändert werden, um so zu überprüfen, ob die Drücke der Messspitze tatsächlich in der Lage sind, die Deckschicht zu unterdrücken.

In atomaren Stick-Slip-Bewegungen werden Energien in der Größenordnung von 0,1 bis $1 \mathrm{eV}$ dissipiert [Gnecco07]. Andere Messmethoden, die diesen Energiebereich untersuchen, könnten so wichtige Informationen liefern, um Dissipationsprozesse zu verstehen. Ramanspektren sind beispielsweise sehr interessant, da man eventuell phononische Anteile der Reibung extrahieren könnte. Im $\mathrm{Pd}_{77,5} \mathrm{Cu}_{6} \mathrm{Si}_{16,5}$-System beispielsweise könnten Phononen die Reibungserhöhung des kristallinen Zustands erklären. Zuletzt wäre es sinnvoll, dabei auch Computersimulationen zu nutzen, um phononische Dissipationskanäle zu simulieren. 


\section{LITERATURVERZEICHNIS}

[Abel07] Abel, D. G.; Krylov, S. Yu. und Frenken, J. W. M.: Evidence for Contact Delocalization in Atomic Scale Friction. Phys. Rev. Lett. (2007), Bd. 99: S. 166102

[Alsem08] Alsem, D. H.; Dugger, M. T.; Stach, E. A. und Ritchie, R. O.: Micron-Scale Friction and Sliding Wear of Polycrystalline Silicon Thin Structural Films in Ambient Air. Journal of Microelectromechanical Systems (2008), Bd. 17(5): S. 1144-1154

[Amontons99] Amontons, Guillaume: De la resistance causée dans les Machines, Tant par les frottemens des parties qui les composent, que par roideur des cordes qu'on y employe, \& la maniere de calculer l'un \& l'autre, in: Histoire de l'Académie royale des sciences, Mémoires de l'Académie royale des sciences (1699), S. 203-222

[Anderson50] Anderson, P. W.: Antiferromagnetism. Theory of Superexchange Interaction. Phys. Rev. (1950), Bd. 79: S. 350-356

[Anderson55] Anderson, P. W. und Hasegawa, H.: Considerations on Double Exchange. Phys. Rev. (1955), Bd. 100: S. 675-681

[Angell88] Angell, C.A.: Perspective on the glass transition. Journal of Physics and Chemistry of Solids (1988), Bd. 49(8): S. $863-871$

[Angell95] Angell, C. A.: Formation of Glasses from Liquids and Biopolymers. Science (1995), Bd. 267(5206): S. 1924-1935

[Bardeen57] Bardeen, J.; Cooper, L. N. und Schrieffer, J. R.: Theory of Superconductivity. Phys. Rev. (1957), Bd. 108: S. 1175-1204

[Barel10a] Barel, Itay; Urbakh, Michael; Jansen, Lars und SchirmeISEN, André: Multibond Dynamics of Nanoscale Friction: The Role of Temperature. Phys. Rev. Lett. (2010), Bd. 104: S. 066104 
[Barel10b] Barel, Itay; Urbakh, Michael; Jansen, Lars und SchirmeiSEN, André: Temperature Dependence of Friction at the Nanoscale: When the Unexpected Turns Normal. Tribology Letters (2010), Bd. 39(3): S. 311-319

[Bednorz86] Bednorz, J. G. und Müller, K. A.: Possible high $T_{\mathrm{C}}$ superconductivity in the BaLaCuO system. Zeitschrift für Physik $B$ Condensed Matter (1986), Bd. 64(2): S. 189-193

[Bedorf10] Bedorf, Dennis und SAmwer, Konrad: Length scale effects on relaxations in metallic glasses. Journal of Non-Crystalline Solids (2010), Bd. 356(6): S. $340-343$

[Bennewitz99] Bennewitz, R.; Gyalog, T.; Guggisberg, M.; Bammerlin, M.; MeYer, E. und GÜntherodt, H.-J.: Atomic-scale stick-slip processes on $\mathrm{Cu}(111)$. Phys. Rev. B (1999), Bd. 60: S. R11301R11304

[Bennewitz15] Bennewitz, Roland: Friction Force Microscopy, in: Enrico Gnecco und Ernst Meyer (Herausgeber) Fundamentals of Friction and Wear on the Nanoscale, Springer International Publishing (2015), S. 3-16

[Bhattachar14] Bhattacharya, Anand und May, Steven J.: Magnetic Oxide Heterostructures. Annual Review of Materials Research (2014), Bd. 44(1): S. 65-90

[Bhushan97] Bhushan, Bharat und Li, Xiaodong: Micromechanical and tribological characterization of doped single-crystal silicon and polysilicon films for microelectromechanical systems devices. Journal of Materials Research (1997), Bd. 12(1): S. 54-63

[Bhushan13] Bhushan, Bharat: Introduction, in: Introduction to Tribology, John Wiley \& Sons, Ltd (2013), S. 1-8

[Binggeli94] BingGeli, M. und Mate, C. M.: Influence of capillary condensation of water on nanotribology studied by force microscopy. Applied Physics Letters (1994), Bd. 65(4): S. 415-417

[Binnig83] Binnig, G. und Rohrer, H.: Scanning tunneling microscopy. Surface Science (1983), Bd. 126(1): S. 236 - 244 
[Binnig86] Binnig, G.; Quate, C. F. und Gerber, Ch.: Atomic Force Microscope. Phys. Rev. Lett. (1986), Bd. 56: S. 930-933

[Björnsso00] Buörnsson, P.; Rübhausen, M.; Bäckström, J.; Käll, M.; ErIKsson, S.; ERIKSEn, J. und BörJesson, L.: Lattice and charge excitations in $\mathrm{La}_{1-x} \mathrm{Sr}_{x} \mathrm{MnO}_{3}$. Phys. Rev. B (2000), Bd. 61: S. 1193-1197

[Bowden42] Bowden, F. P. und Tabor, D.: Mechanism of Metallic Friction. Nature (1942), Bd. 150: S. 197

[Bowden59] Bowden, F. P.; Tabor, D. und Übersetzt von Freitag, E. H.: Der Mechanismus der metallischen Reibung, in: Reibung und Schmierung fester Körper, Springer Berlin Heidelberg, Berlin, Heidelberg (1959), S. 111-146

[Bragg13] BragG, W. L und BragG, W. H.: The reflection of X-rays by crystals, in: Proc. R. Soc. Lond. A, Bd. 88, The Royal Society, S. 428-438

[Brentano24] Brentano, J: Focussing method of crystal powder analysis by X-rays. Proceedings of the Physical Society of London (1924), Bd. 37(1): S. 184

[Bruker AXS01] BRUker AXS GmBH: D88 Advance Röntgendiffraktometer Handbuch (2001)

[Burgy01] Burgy, J.; Mayr, M.; Martin-Mayor, V.; Moreo, A. und Dagotto, E.: Colossal Effects in Transition Metal Oxides Caused by Intrinsic Inhomogeneities. Phys. Rev. Lett. (2001), Bd. 87: S. 277202

[Burnham93] Burnham, N A; Colton, R J und Pollock, H M: Interpretation of force curves in force microscopy. Nanotechnology (1993), Bd. 4(2): S. 64

[Calderón99] Calderón, M. J.; Brey, L. und Guinea, F.: Surface electronic structure and magnetic properties of doped manganites. Phys. Rev. B (1999), Bd. 60: S. 6698-6704 
[Cappella99] Cappella, B. und Dietler, G.: Force-distance curves by atomic force microscopy. Surface Science Reports (1999), Bd. 34(1): S. 1 $-104$

[Caron09] Caron, A. und Arnold, W.: Observation of local internal friction and plasticity onset in nanocrystalline nickel by atomic force acoustic microscopy. Acta Materialia (2009), Bd. 57(15): S. 4353 $-4363$

[Chahara93] Chahara, Ken-ichi; Ohno, Toshiyuki; Kasai, Masahiro und Kozono, Yuzoo: Magnetoresistance in magnetic manganese oxide with intrinsic antiferromagnetic spin structure. Applied Physics Letters (1993), Bd. 63(14): S. 1990-1992

[Charles02] Charles, Kittel: Einführung in die Festkörperphysik, De Gruyter (2002)

[Chumakov04] Chumakov, A. I.; Sergueev, I.; van Bürck, U.; SchirmaCher, W.; Asthalter, T.; Rüffer, R.; Leupold, O. und Petry, W.: Collective Nature of the Boson Peak and Universal Transboson Dynamics of Glasses. Phys. Rev. Lett. (2004), Bd. 92: S. 245508

[Cieplak94] Cieplak, Marek; Smith, Elizabeth D. und RobBins, Mark O.: Molecular Origins of Friction: The Force on Adsorbed Layers. Science (1994), Bd. 265(5176): S. 1209-1212

[Coulomb73] Coulomb, CA: Essai sur une application des règles de maximis et minimis à quelques problèmes de statique relatifs à l'architecture. Memoires de math. et de phys. pres. Royale des Sciences (1773): S. 343

[Coulomb97] De Coulomb, Charles Augustin: Memoire sur les frottemens de la pointe des pivots, Institut de France (1797)

[Coulomb01] DE Coulomb, Charles Augustin: Expériences destinées à déterminer la cohérence des fluides et les lois de leur résistance dans les mouvemens très-lents (1801) 
[Coulomb21] DE Coulomb, Charles Augustin: Théorie des machines simples en ayant égard au frottement de leurs parties et à la roideur des cordages, Bachelier (1821)

[Cretin93] Cretin, B. und Sthal, F.: Scanning microdeformation microscopy. Applied Physics Letters (1993), Bd. 62(8): S. 829-831

[Daillant08] Daillant, J. und Gibaud, A.: X-ray and Neutron Reflectivity: Principles and Applications, Lecture Notes in Physics, Springer, Berlin, Heidelberg (2008)

[Daly96] Daly, C. und Krim, J.: Sliding Friction of Solid Xenon Monolayers and Bilayers on $\operatorname{Ag}(111)$. Phys. Rev. Lett. (1996), Bd. 76: S. 803-806

[Dang-Hyok14] Dang-Hyok, Yoon; Raju, Kati; Bong-KI, Min und Reddy, Paduru Venugopal: Synthesis and characterization of microwave sintered ferromagnetic-ferroelectric perovskite composites. Ceramics International (2014), Bd. 40(8): S. 13497 - 13505

[Debenedett01] Debenedetti, Pablo G. und Stillinger, Frank H.: Supercooled liquids and the glass transition. Nature (2001), Bd. 410(6825): S. 259-267

[Deng92] Deng, Keren und Ko, Wen H: A study of static friction between silicon and silicon compounds. Journal of Micromechanics and Microengineering (1992), Bd. 2(1): S. 14

[Derjaguin75] Derjaguin, B.V; Muller, V.M und Toporov, Yu.P: Effect of contact deformations on the adhesion of particles. Journal of Colloid and Interface Science (1975), Bd. 53(2): S. $314-326$

[Desagulier24] Desaguliers, J. T.: Some Experiments concerning the Cohesion of Lead, by the Same. Philosophical Transactions (1683-1775) (1724), Bd. 33: S. 345-347

[Dienwiebel04] Dienwiebel, Martin; Verhoeven, Gertjan S.; PradeeP, Namboodiri; Frenken, Joost W. M.; Heimberg, Jennifer A. und Zandbergen, Henny W.: Superlubricity of Graphite. Phys. Rev. Lett. (2004), Bd. 92: S. 126101 
[Dildar12] Dildar, I. Mubeen; Beekman, C.; He, Xin und Aarts, J.: Hall effect measurements on strained and unstrained thin films of $\mathrm{La}_{0.7} \mathrm{Ca}_{0.3} \mathrm{MnO}_{3}$ and $\mathrm{La}_{0.7} \mathrm{Sr}_{0.3} \mathrm{MnO}_{3}$. Phys. Rev. $B$ (2012), Bd. 85: S. 205103

[Dinelli97] Dinelli, F.; Biswas, S. K.; Briggs, G. A. D. und Kolosov, O. V.: Ultrasound induced lubricity in microscopic contact. Applied Physics Letters (1997), Bd. 71(9): S. 1177-1179

[Dinelli00] Dinelli, F.; Castell, M. R.; Ritchie, D. A.; Mason, N. J.; Briggs, G. A. D. und Kolosov, O. V.: Mapping surface elastic properties of stiff and compliant materials on the nanoscale using ultrasonic force microscopy. Philosophical Magazine A (2000), Bd. 80(10): S. 2299-2323

[Evans01] Evans, Evan: Probing the Relation Between Force-Lifetime-and Chemistry in Single Molecular Bonds. Annual Review of Biophysics and Biomolecular Structure (2001), Bd. 30(1): S. 105-128, pMID: 11340054

[Fajardo14] Fajardo, O. Y.; Gnecco, E. und Mazo, J. J.: Out-of-plane and in-plane actuation effects on atomic-scale friction. Phys. Rev. B (2014), Bd. 89: S. 075423

[Fall14] Fall, A.; Weber, B.; Pakpour, M.; Lenoir, N.; Shahidzadeh, N.; Fiscina, J.; Wagner, C. und Bonn, D.: Sliding Friction on Wet and Dry Sand. Phys. Rev. Lett. (2014), Bd. 112: S. 175502

[Fang01] FAng, Zhong und Terakura, Kiyoyuki: Surface Magnetic Phase Diagram of Tetragonal Manganites. Journal of the Physical Society of Japan (2001), Bd. 70(11): S. 3356-3361

[Filippetti00] Filippetti, Alessio und Pickett, Warren E.: Double-exchangedriven spin pairing at the (001) surface of manganites. Phys. Rev. B (2000), Bd. 62: S. 11571-11575

[Filippov04] Filippov, A. E.; Klafter, J. und Urbakh, M.: Friction through Dynamical Formation and Rupture of Molecular Bonds. Phys. Rev. Lett. (2004), Bd. 92: S. 135503 
[Friedrich13] Friedrich, W.; KNIPPING, P. und Laue, M.: Interferenzerscheinungen bei Röntgenstrahlen. Annalen der Physik (1913), Bd. 346(10): S. 971-988

[Fujiwara07] FujIwARA, Hiroyuki: Spectroscopic Ellipsometry, John Wiley \& Sons, Ltd (2007)

[Fulcher25] Fulcher, Gordon S.: ANALYSIS OF RECENT MEASUREMENTS OF THE VISCOSITY OF GLASSES. Journal of the American Ceramic Society (1925), Bd. 8(6): S. 339-355

[Gillmor71] Gillmor, C. Stewart: Coulomb and the Evolution of Physics and Engineering in Eighteenth-Century France, Princeton University Press (1971)

[Gnecco00] Gnecco, E.; Bennewitz, R.; Gyalog, T.; Loppacher, Ch.; Bammerlin, M.; Meyer, E. und Güntherodt, H.-J.: Velocity Dependence of Atomic Friction. Phys. Rev. Lett. (2000), Bd. 84: S. $1172-1175$

[Gnecco07] Gnecco, E. und Meyer, E.: Fundamentals of Friction and Wear, NanoScience and Technology, Springer Berlin Heidelberg (2007)

[Gnecco08] Gnecco, Enrico; Maier, Sabine und Meyer, Ernst: Superlubricity of dry nanocontacts. Journal of Physics: Condensed Matter (2008), Bd. 20(35): S. 354004

[Golding72] Golding, Brage; Bagley, B. G. und Hsu, F. S. L.: Soft Transverse Phonons in a Metallic Glass. Phys. Rev. Lett. (1972), Bd. 29: S. $68-70$

[Goldschmid26] Goldschmidt, V. M.: Die Gesetze der Krystallochemie. Naturwissenschaften (1926), Bd. 14(21): S. 477-485

[Goldschmid43] Goldschmidt, H. J. und RAIT, J. R.: Silicates of the Perovskite Type of Structure. Nature (1943), Bd. 152: S. 356 EP -

[Goldstein69] Goldstein, Martin: Viscous Liquids and the Glass Transition: A Potential Energy Barrier Picture. The Journal of Chemical Physics (1969), Bd. 51(9): S. 3728-3739 
[Goncharuk13] Goncharuk, I. N.; Ilinskiy, A. V.; Kvashenkina, O. E. und SHADRIN, E. B.: Electron-electron correlations in Raman spectra of $\mathrm{VO}_{2}$. Physics of the Solid State (2013), Bd. 55(1): S. 164-174

[Goodenough55] Goodenough, John B.: Theory of the Role of Covalence in the Perovskite-Type Manganites [La, M(II)] $\mathrm{MnO}_{3}$. Phys. Rev. (1955), Bd. 100: S. 564-573

[Gross04] Gross, R. und Marx, A.: Der Kolossale Magnetowiderstandseffekt, in: Vorlesungsskript: Spinelektronik, Kap. 4, WaltherMeissner-Institut - Lehrstuhl für Technische Physik (2004), S. 95-136

[Gross12] Gross, R. und Marx, A.: Festkörperphysik, Oldenbourg Wissenschaftsverlag (2012)

[Guan10] Guan, Pengfei; Chen, Mingwei und Egami, Takeshi: StressTemperature Scaling for Steady-State Flow in Metallic Glasses. Phys. Rev. Lett. (2010), Bd. 104: S. 205701

[Hachenberg06] HaCHEnBERG, Jörg: Mechanische Spektroskopie an metallischen Gläsern und Copolymeren, Dissertation, Georg-AugustUniversität Göttingen (2006)

[Hao91] Hao, Huang Wen; Baró, A. M. und SÁenz, J. J.: Electrostatic and contact forces in force microscopy. Journal of Vacuum Science 8 Technology B: Microelectronics and Nanometer Structures Processing, Measurement, and Phenomena (1991), Bd. 9(2): S. 13231328

[Hartinger04] Hartinger, Ch.; Mayr, F.; Deisenhofer, J.; Loidl, A. und Kopp, T.: Large and small polaron excitations in $\mathrm{La}_{2 / 3}(\mathrm{Sr} / \mathrm{Ca})_{1 / 3} \mathrm{MnO}_{3}$ films. Phys. Rev. $B$ (2004), Bd. 69: S. 100403

[Hartinger06] Hartinger, Ch.; Mayr, F.; Loidl, A. und Kopp, T.: Polaronic excitations in colossal magnetoresistance manganite films. Phys. Rev. B (2006), Bd. 73: S. 024408 
[Hell94] Hell, Stefan W. und Wichmann, Jan: Breaking the diffraction resolution limit by stimulated emission: stimulated-emissiondepletion fluorescence microscopy. Opt. Lett. (1994), Bd. 19(11): S. 780-782

[Helmolt93] von Helmolt, R.; Wecker, J.; Holzapfel, B.; Schultz, L. und SAMWER, K.: Giant negative magnetoresistance in perovskitelike $\mathrm{La}_{2 / 3} \mathrm{Ba}_{1 / 3} \mathrm{MnO}_{x}$ ferromagnetic films. Phys. Rev. Lett. (1993), Bd. 71: S. 2331-2333

[Hemberger02] Hemberger, J.; Krimmel, A.; Kurz, T.; Krug von Nidda, H.-A.; Ivanov, V. Yu.; Mukhin, A. A.; Balbashov, A. M. und LoIDL, A.: Structural, magnetic, and electrical properties of single-crystalline $\mathrm{La}_{1-x} \mathrm{Sr}_{x} \mathrm{MnO}_{3}(0.4<x<0.85)$. Phys. Rev. B (2002), Bd. 66: S. 094410

[Hesjedal01] Hesjedal, T. und Behme, G.: High-resolution imaging of surface acoustic wave scattering. Applied Physics Letters (2001), Bd. 78(13): S. 1948-1950

[Heslot94] Heslot, F.; Baumberger, T.; Perrin, B.; Caroli, B. und Caroli, C.: Creep, stick-slip, and dry-friction dynamics: Experiments and a heuristic model. Phys. Rev. E (1994), Bd. 49: S. 4973-4988

[Hirsekorn95] Hirsekorn, S.; Pangraz, S.; Weides, G. und Arnold, W.: Measurement of elastic impedance with high spatial resolution using acoustic microscopy. Applied Physics Letters (1995), Bd. 67(6): S. 745-747

[Hirsekorn97] Hirsekorn, S; RABE, U und ARnold, W: Theoretical description of the transfer of vibrations from a sample to the cantilever of an atomic force microscope. Nanotechnology (1997), Bd. 8(2): S. 57

[Holmberg12] Holmberg, Kenneth; Andersson, Peter und Erdemir, Ali: Global energy consumption due to friction in passenger cars. Tribology International (2012), Bd. 47: S. 221 - 234

[Hölscher08] Hölscher, Hendrik; Schirmeisen, André und Schwarz, Udo D: Principles of atomic friction: from sticking atoms to su- 
perlubric sliding. Philosophical Transactions of the Royal Society of London A: Mathematical, Physical and Engineering Sciences (2008), Bd. 366(1869): S. 1383-1404

[Hwang95] Hwang, H. Y.; Cheong, S-W.; Radaelli, P. G.; Marezio, M. und BAtlogG, B.: Lattice Effects on the Magnetoresistance in Doped $\mathrm{LaMnO}_{3}$. Phys. Rev. Lett. (1995), Bd. 75: S. 914-917

[Iizuka09] Irzuka, Hiroyuki; NAkamura, Jun und Natori, Akiko: Control mechanism of friction by dynamic actuation of nanometer-sized contacts. Phys. Rev. B (2009), Bd. 80: S. 155449

[Israelachv72] Israelachvili, J. N. und TABOR, D.: The measurement of van der Waals dispersion forces in the range 1.5 to $130 \mathrm{~nm}$. Proceedings of the Royal Society of London A: Mathematical, Physical and Engineering Sciences (1972), Bd. 331(1584): S. 19-38

[Israelachv73] IsRaelachvili, J. N. und TABor, D.: Shear Properties of Molecular Films. Nature Physical Science (1973), Bd. 241: S. 148 EP

[Jahn37] JAhn, H. A. und TELLER, E.: Stability of polyatomic molecules in degenerate electronic states - I-Orbital degeneracy. Proceedings of the Royal Society of London A: Mathematical, Physical and Engineering Sciences (1937), Bd. 161(905): S. 220-235

[Jansen10] Jansen, Lars; Hölscher, Hendrik; Fuchs, Harald und SchiRMEISEn, André: Temperature Dependence of Atomic-Scale StickSlip Friction. Phys. Rev. Lett. (2010), Bd. 104: S. 256101

[Jinesh06] Jinesh, K. B. und Frenken, J. W. M.: Capillary Condensation in Atomic Scale Friction: How Water Acts like a Glue. Phys. Rev. Lett. (2006), Bd. 96: S. 166103

[Jinesh08] Jinesh, K. B.; Krylov, S. Yu.; Valk, H.; Dienwiebel, M. und Frenken, J. W. M.: Thermolubricity in atomic-scale friction. Phys. Rev. B (2008), Bd. 78: S. 155440

[Johnson71] Johnson, K. L.; Kendall, K. und Roberts, A. D.: Surface energy and the contact of elastic solids. Proceedings of the Roy- 
al Society of London A: Mathematical, Physical and Engineering Sciences (1971), Bd. 324(1558): S. 301-313

[Johnson85] Johnson, K. L.: Contact Mechanics, Cambridge University Press (1985)

[Johnson05] Johnson, W. L. und SAmwer, K.: A Universal Criterion for Plastic Yielding of Metallic Glasses with a $\left(T / T_{g}\right)^{2 / 3}$ Temperature Dependence. Phys. Rev. Lett. (2005), Bd. 95: S. 195501

[Jonker50] JonkeR, G.H. und SAnten, J.H. Van: Ferromagnetic compounds of manganese with perovskite structure. Physica (1950), Bd. 16(3): S. 337 - 349

[Jost66] Jost, Peter: Lubrication (tribology), education and research: a report on the present position and industry's needs, Great Britain. Dept. of Education and Science, H. M. Stationery Off. (1966)

[Jungbauer14] Jungbauer, M.; Hühn, S.; Egoavil, R.; TAN, H.; VerbeecK, J.; Tendeloo, G. Van und Moshnyaga, V.: Atomic layer epitaxy of Ruddlesden-Popper $\mathrm{SrO}(\mathrm{SrTiO} 3) n$ films by means of metalorganic aerosol deposition. Applied Physics Letters (2014), Bd. 105(25): S. 251603

[Kanamori59] KanAmORI, Junjiro: Superexchange interaction and symmetry properties of electron orbitals. Journal of Physics and Chemistry of Solids (1959), Bd. 10(2): S. $87-98$

[Kang11] KAng, Manil; KIM, Inkoo; KIM, Sok Won; RYU, Ji-Wook und PARK, Hyo Yeol: Metal-insulator transition without structural phase transition in V2O5 film. Applied Physics Letters (2011), Bd. 98(13): S. 131907

[Kerssemake98] Kerssemakers, J. und Hosson, J.Th.M. De: Probing the interface potential in stick/slip friction by a lateral force modulation technique. Surface Science (1998), Bd. 417(2): S. 281 - 291

[Killgore11] Killgore, J. P.; Yablon, D. G.; Tsou, A. H.; Gannepalli, A.; Yuya, P. A.; Turner, J. A.; Proksch, R. und Hurley, D. C.: Viscoelastic Property Mapping with Contact Resonance 
Force Microscopy. Langmuir (2011), Bd. 27(23): S. 13983-13987, pMID: 22054300

[Kim16] Kim, Jong Hun; Fu, Deyi; Kwon, Sangku; LiU, Kai; Wu, Junqiao und PARK, Jeong Young: Crossing Thermal Lubricity and Electronic Effects in Friction: Vanadium Dioxide under the Metal-Insulator Transition. Advanced Materials Interfaces (2016), Bd. 3(2): S. 1500388-n/a, 1500388

[Kisiel11] Kisiel, Marcin; Gnecco, Enrico; Gysin, Urs; Marot, Laurent; Rast, Simon und Meyer, Ernst: Suppression of electronic friction on $\mathrm{Nb}$ films in the superconducting state. Nature Materials (2011), Bd. 10: S. 119-122

[Klar99] Klar, Thomas A. und Hell, Stefan W.: Subdiffraction resolution in far-field fluorescence microscopy. Opt. Lett. (1999), Bd. 24(14): S. 954-956

[Kolosov93] Kolosov, Oleg und Yamanaka, Kazushi: Nonlinear Detection of Ultrasonic Vibrations in an Atomic Force Microscope. Japanese Journal of Applied Physics (1993), Bd. 32(8A): S. L1095

[Kopycinska08] Kopycinska-Müller, M; Caron, A; Hirsekorn, S; Rabe, U; Natter, Harald; Hempelmann, Rolf; Birringer, Rainer und ARnolD, Walter: Quantitative Evaluation of Elastic Properties of Nano-Crystalline Nickel Using Atomic Force Acoustic Microscopy. Zeitschrift für Physikalische Chemie (2008), Bd. 222(2-3/2008): S. 471-498

[Kovaleva10] Kovaleva, N. N.; Oleś, Andrzej M.; Balbashov, A. M.; MalJuk, A.; Argyriou, D. N.; Khaliullin, G. und Keimer, B.: Low-energy Mott-Hubbard excitations in $\mathrm{LaMnO}_{3}$ probed by optical ellipsometry. Phys. Rev. B (2010), Bd. 81: S. 235130

[Krausser15] Krausser, Johannes; SAmwer, Konrad H. und ZaCCOnE, Alessio: Interatomic repulsion softness directly controls the fragility of supercooled metallic melts. Proceedings of the National Academy of Sciences (2015), Bd. 112(45): S. 13762-13767 
[Krim88] Krim, J. und Widom, A.: Damping of a crystal oscillator by an adsorbed monolayer and its relation to interfacial viscosity. Phys. Rev. B (1988), Bd. 38: S. 12184-12189

[Krim90] Krim, J.; Watts, E. T. und Digel, J.: Slippage of simple liquid films adsorbed on silver and gold substrates. Journal of Vacuum Science \& Technology A: Vacuum, Surfaces, and Films (1990), Bd. 8(4): S. 3417-3420

[Krim91a] Krim, J. und Chiarello, R.: Sliding friction measurements of molecularly thin films. Journal of Vacuum Science \&5 Technology A: Vacuum, Surfaces, and Films (1991), Bd. 9(4): S. 2566-2569

[Krim91b] Krim, J.; Solina, D. H. und Chiarello, R.: Nanotribology of a Kr monolayer: A quartz-crystal microbalance study of atomicscale friction. Phys. Rev. Lett. (1991), Bd. 66: S. 181-184

[Krylov05] Krylov, S. Yu.; Jinesh, K. B.; Valk, H.; Dienwiebel, M. und Frenken, J. W. M.: Thermally induced suppression of friction at the atomic scale. Phys. Rev. E (2005), Bd. 71: S. 065101

[Krylov06] Krylov, S. Yu.; Dijksman, J. A.; van Loo, W. A. und FrenKEN, J. W. M.: Stick-Slip Motion in Spite of a Slippery Contact: Do We Get What We See in Atomic Friction? Phys. Rev. Lett. (2006), Bd. 97: S. 166103

[Krylov07] Krylov, Sergey Yu und Frenken, Joost W M: Thermal contact delocalization in atomic scale friction: a multitude of friction regimes. New Journal of Physics (2007), Bd. 9(10): S. 398

[Krylov14] Krylov, Sergey Yu. und Frenken, Joost W. M.: The physics of atomic-scale friction: Basic considerations and open questions. physica status solidi (b) (2014), Bd. 251(4): S. 711-736

[Lemons74] Lemons, R. A. und Quate, C. F.: Acoustic microscope-scanning version. Applied Physics Letters (1974), Bd. 24(4): S. 163-165

[Liu05] Liu, Li; Pang, Shujie; Ma, Chaoli und Zhang, Tao: Effect of Minor Au Addition on Glass-Forming Ability and Mechanical Properties of PdCuAuSiP Alloys. MATERIALS TRANSACTIONS (2005), Bd. 46(12): S. 2945-2948 
[Lobad00] Lobad, Ahmed I.; Averitt, Richard D.; Kwon, Chuhee und TAYLOR, Antoinette J.: Spin-lattice interaction in colossal magnetoresistance manganites. Applied Physics Letters (2000), Bd. 77(24): S. 4025-4027

[Lunkenheim00] Lunkenheimer, P.; Schneider, U.; Brand, R. und Loid, A.: Glassy dynamics. Contemporary Physics (2000), Bd. 41(1): S. 1536

[Ma18] Ma, C.; Pfahl, V.; Chen, Y.; Chu, J.; Phani, K.; Kumar, A.; Arnold, W. und Samwer, K.: Stick-to-Sliding Transition in Contact-Resonance Atomic Force Microscopy (2018), akzeptiert bei Applied Physics Letters (31.07.18)

[Macke14] Macke, Sebastian; RAdi, Abdullah; Hamann-Borrero, Jorge E.; Verna, Adriano; Bluschke, Martin; BrüCK, Sebastian; Goering, Eberhard; Sutarto, Ronny; He, Feizhou; CristiaNI, Georg; Wu, Meng; Benckiser, Eva; Habermeier, HannsUlrich; Logvenov, Gennady; Gauquelin, Nicolas; Botton, Gianluigi A.; Kajdos, Adam P.; Stemmer, Susanne; SawatzKY, Georg A.; Haverkort, Maurits W.; KeImer, Bernhard und Hinkov, Vladimir: Element Specific Monolayer Depth Profiling. Advanced Materials (2014)

[Maier05] Maier, S.; Sang, Yi; Filleter, T.; Grant, M.; Bennewitz, R.; Gnecco, E. und Meyer, E.: Fluctuations and jump dynamics in atomic friction experiments. Phys. Rev. B (2005), Bd. 72: S. 245418

[Mannella04] Mannella, N.; Rosenhahn, A.; Booth, C. H.; Marchesini, S.; Mun, B. S.; Yang, S.-H.; Ibrahim, K.; TomiokA, Y. und FADley, C. S.: Direct Observation of High-Temperature Polaronic Behavior in Colossal Magnetoresistive Manganites. Phys. Rev. Lett. (2004), Bd. 92: S. 166401

[Mannella05] Mannella, N.; Rosenhahn, A.; Watanabe, M.; Sell, B.; Nambu, A.; Ritchey, S.; Arenholz, E.; Young, A.; Tomioka, Y. und FADley, C. S.: Temperature-dependent X-ray absorption 
spectroscopy of colossal magnetoresistive perovskites. Phys. Rev. $B$ (2005), Bd. 71: S. 125117

[Martin87a] Martin, Y. und Wickramasinghe, H. K.: Magnetic imaging by "force microscopy"with $1000 \AA$ resolution. Applied Physics Letters (1987), Bd. 50(20): S. 1455-1457

[Martin87b] Martin, Y.; Williams, C. C. und Wickramasinghe, H. K.: Atomic force microscope-force mapping and profiling on a sub $100 \AA$ scale. Journal of Applied Physics (1987), Bd. 61(10): S. $4723-4729$

[Mate87] Mate, C. Mathew; McClelland, Gary M.; Erlandsson, Ragnar und ChIAng, Shirley: Atomic-scale friction of a tungsten tip on a graphite surface. Phys. Rev. Lett. (1987), Bd. 59: S. 19421945

[Mathur03] Mathur, Neil und Littlewood, Peter: Mesoscopic Texture in Manganites. Physics Today (2003), Bd. 56(1): S. 25-30

[Mazeran08] Mazeran, Pierre-Emmanuel und Beyaoui, Moez: Initiation of Sliding of an Elastic Contact at a Nanometer Scale Under a Scanning Force Microscope Probe. Tribology Letters (2008), Bd. 30(1): S. 1-11

[McClelland87] McClelland, Gary M.; Erlandsson, Ragnar und Chiang, Shirley: Atomic Force Microscopy: General Principles and a New Implementation, in: Donald O. Thompson und Dale E. Chimenti (Herausgeber) Review of Progress in Quantitative Nondestructive Evaluation, Springer US, Boston, MA (1987), S. 1307-1314

[Meer16] MEER, Hendrik: Reibungskraftmessungen an VanadiumdioxidDünnfilmen, Bachelorarbeit, Georg-August-Universität Göttingen (2016)

[Megaw46] MEgaw, Helen D: Crystal structure of double oxides of the perovskite type. Proceedings of the Physical Society (1946), Bd. 58(2): S. 133 
[Meyer88] Meyer, Gerhard und Amer, Nabil M.: Novel optical approach to atomic force microscopy. Applied Physics Letters (1988), Bd. 53(12): S. 1045-1047

[Meyer99] Meyer, A.; Busch, R. und Schober, H.: Time-Temperature Superposition of Structural Relaxation in a Viscous Metallic Liquid. Phys. Rev. Lett. (1999), Bd. 83: S. 5027-5029

[Miller39] Miller, W.H.: A Treatise on Crystallography, For J. \& J. J. Deighton (1839)

[Millis95] Millis, A. J.; Littlewood, P. B. und Shraiman, B. I.: Double Exchange Alone Does Not Explain the Resistivity of $\mathrm{La}_{1-x} \mathrm{Sr}_{x} \mathrm{MnO}_{3}$. Phys. Rev. Lett. (1995), Bd. 74: S. 5144-5147

[Millis98] Millis, A. J.: Lattice effects in magnetoresistive manganese perovskites. Nature (1998), Bd. 392: S. 147 EP -

[Momma11] Momma, Koichi und Izumi, Fujio: VESTA3 for three-dimensional visualization of crystal, volumetric and morphology data. Journal of Applied Crystallography (2011), Bd. 44(6): S. 1272-1276

[Morita15] Morita, S.; Giessibl, F.J.; Meyer, E. und Wiesendanger, R.: Noncontact Atomic Force Microscopy, Nr. Bd. 3 in NanoScience and Technology, Springer International Publishing (2015)

[Moshnyaga99] Moshnyaga, V.; Khoroshun, I.; Sidorenko, A.; PetrenKO, P.; Weidinger, A.; Zeitler, M.; Rauschenbach, B.; Tidecks, R. und SAMwER, K.: Preparation of rare-earth manganite-oxide thin films by metalorganic aerosol deposition technique. Applied Physics Letters (1999), Bd. 74(19): S. 28422844

[Moshnyaga07] Moshnyaga, Vasily und Samwer, Konrad: Ferromagnetic Manganite Films, in: Handbook of Magnetism and Advanced Magnetic Materials, John Wiley \& Sons, Ltd (2007), S. 1-29

[Moshnyaga11] Moshnyaga, V. und Samwer, K.: Electron-lattice correlations and phase transitions in CMR manganites. Annalen der Physik (2011), Bd. 523(8-9): S. 652-663 
[Moshnyaga14] Moshnyaga, V.; Belenchuk, A.; Hühn, S.; Kalkert, C.; Jungbauer, M.; Lebedev, O. I.; Merten, S.; Choi, K.-Y.; Lemmens, P.; Damaschke, B. und Samwer, K.: Intrinsic antiferromagnetic coupling underlies colossal magnetoresistance effect: Role of correlated polarons. Phys. Rev. B (2014), Bd. 89: S. 024420

[Müller08] Müller, Georg M.; Walowski, Jakob; Djordjevic, Marija; Miao, Gou-Xing; Gupta, Arunava; Ramos, Ana V.; Gehrke, Kai; Moshnyaga, Vasily; Samwer, Konrad; Schmalhorst, Jan; Thomas, Andy; Hütten, Andreas; Reiss, Günter; MooDERA, Jagadeesh S. und MünZEnBerg, Markus: Spin polarization in half-metals probed by femtosecond spin excitation. Nature Materials (2008), Bd. 8: S. 56

[Murakami98] Murakami, Y.; Hill, J. P.; Gibbs, D.; Blume, M.; Koyama, I.; Tanaka, M.; Kawata, H.; Arima, T.; Tokura, Y.; HiroTA, K. und Endoh, Y.: Resonant X-Ray Scattering from Orbital Ordering in $\mathrm{LaMnO}_{3}$. Phys. Rev. Lett. (1998), Bd. 81: S. 582-585

[Murrel193] Murrell, M. P.; Welland, M. E.; O’Shea, S. J.; Wong, T. M. H.; Barnes, J. R.; McKinnon, A. W.; Heyns, M. und VERHAVERBEKE, S.: Spatially resolved electrical measurements of $\mathrm{SiO}_{2}$ gate oxides using atomic force microscopy. Applied Physics Letters (1993), Bd. 62(7): S. 786-788

[Müser03] Müser, Martin H.; Urbakh, Michael und Robbins, Mark O.: Statistical Mechanics of Static and Low-Velocity Kinetic Friction, in: Advances in Chemical Physics, John Wiley \& Sons, Inc. (2003), S. $187-272$

[Newberry93] NewberRY, Percy Edward; FrASER, George Willoughby ET AL.: El Bersheh, Part 1,(The tomb of Tehuti-Hetep) (1893)

[O'Connel197] O'Connell, P. A. und McKenna, G. B.: Large deformation response of polycarbonate: Time-temperature, time-aging time, and time-strain superposition. Polymer Engineering \& Science (1997), Bd. 37(9): S. 1485-1495 
[Okazaki04] Okazaki, K.; Wadati, H.; Fujimori, A.; Onoda, M.; MuRAOKA, Y. und HiroI, Z.: Photoemission study of the metalinsulator transition in $\mathrm{VO}_{2} / \mathrm{TiO}_{2}(001)$ : Evidence for strong electron-electron and electron-phonon interaction. Phys. Rev. B (2004), Bd. 69: S. 165104

[Park06] Park, Jeong Young; Ogletree, D. F.; Thiel, P. A. und SalMERON, M.: Electronic Control of Friction in Silicon pn Junctions. Science (2006), Bd. 313(5784): S. 186

[Persson91] Persson, B. N. J.: Surface resistivity and vibrational damping in adsorbed layers. Phys. Rev. B (1991), Bd. 44: S. 3277-3296

[Persson95] Persson, B. N. J. und Volokitin, A. I.: Electronic friction of physisorbed molecules. The Journal of Chemical Physics (1995), Bd. 103(19): S. 8679-8683

[Persson00] Persson, B.: Sliding Friction: Physical Principles and Applications, NanoScience and Technology, Springer Berlin Heidelberg (2000)

[Persson03] Persson, B.N.J.; Albohr, O.; Mancosu, F.; Peveri, V.; SAMoILOV, V.N. und SiveBAEK, I.M.: On the nature of the static friction, kinetic friction and creep. Wear (2003), Bd. 254(9): S. 835 - 851, papers presented at the 280th We-Hereaus Seminar Integrating Friction and Wear Research

[Pfahl17] Pfahl, V.; Phani, M. K.; Büchsenschütz-GöBeler, M.; KUmar, A.; Moshnyaga, V.; Arnold, W. und Samwer, K.: Conduction electrons as dissipation channel in friction experiments at the metal-metal transition of LSMO measured by contactresonance atomic force microscopy. Applied Physics Letters (2017), Bd. 110(5): S. 053102

[Pfahl18] Pfahl, V.; Ma, C.; Arnold, W. und Samwer, K.: Universal aspects of sonolubrication in amorphous and crystalline materials. Journal of Applied Physics (2018), Bd. 123(3): S. 035301

[Phani16] Phani, M. Kalyan; Kumar, Anish; Arnold, W. und Samwer, K.: Elastic stiffness and damping measurements in titanium al- 
loys using atomic force acoustic microscopy. Journal of Alloys and Compounds (2016), Bd. 676(Supplement C): S. 397 - 406

[Piner97] Piner, Richard D. und Mirkin, Chad A.: Effect of Water on Lateral Force Microscopy in Air. Langmuir (1997), Bd. 13(26): S. 6864-6868

[Podobedov98] Podobedov, V. B.; Weber, A.; Romero, D. B.; Rice, J. P. und Drew, H. D.: Effect of structural and magnetic transitions in $\mathrm{La}_{1-x} M_{x} \mathrm{MnO}_{3}(M=\mathrm{Sr}, \mathrm{Ca})$ single crystals in Raman scattering. Phys. Rev. B (1998), Bd. 58: S. 43-46

[Popova15] Popova, Elena und Popov, Valentin L.: The research works of Coulomb and Amontons and generalized laws of friction. Friction (2015), Bd. 3(2): S. 183-190

[Prandtl28] PrandtL, L.: Ein Gedankenmodell zur kinetischen Theorie der festen Körper. ZAMM - Journal of Applied Mathematics and Mechanics / Zeitschrift für Angewandte Mathematik und Mechanik (1928), Bd. 8(2): S. 85-106

[Qi08] QI, Yabing; Park, J. Y.; Hendriksen, B. L. M.; Ogletree, D. F. und SAlmeron, M.: Electronic contribution to friction on GaAs: An atomic force microscope study. Phys. Rev. B (2008), Bd. 77: S. 184105

[Quijada98] QuiJada, M.; Cerne, J.; Simpson, J. R.; Drew, H. D.; Ahn, K. H.; Millis, A. J.; Shreekala, R.; Ramesh, R.; Rajeswari, M. und Venkatesan, T.: Optical conductivity of manganites: Crossover from Jahn-Teller small polaron to coherent transport in the ferromagnetic state. Phys. Rev. B (1998), Bd. 58: S. 16093 16102

[Rabe94] RABE, U. und Arnold, W.: Acoustic microscopy by atomic force microscopy. Applied Physics Letters (1994), Bd. 64(12): S. 14931495

[Rabe96] Rabe, U.; Janser, K. und Arnold, W.: Vibrations of free and surface-coupled atomic force microscope cantilevers: Theory and 
experiment. Review of Scientific Instruments (1996), Bd. 67(9): S. $3281-3293$

[Rabe02] Rabe, U; Kopycinska-Müller, M; Reinstädtler, M; HirseKORN, S und ARnold, W: Nonlinear Effects in Ultrasonic Transmission in Atomic Force Microscope Contacts, in: O. V. Rudenko und O. A. Sapozhnikov (Herausgeber) Proc. 16th Int. Symp. Nonlinear Acoustics Moscow, Faculty of Physics, Moscow State University, Bd. 2, S. 711-718

[Rabe06] RaBe, Ute: Atomic Force Acoustic Microscopy, in: Harald Bhushan, Bharatand Fuchs (Herausgeber) Applied Scanning Probe Methods II: Scanning Probe Microscopy Techniques, Kap. 2, Springer Berlin Heidelberg, Berlin, Heidelberg (2006), S. 37-90

[Ray03] RAY, A und DEY, T.K: Thermal conductivity of $\mathrm{La}_{0.67}\left(\mathrm{Ca}_{x} \mathrm{Sr}_{1-x}\right)_{0.33} \mathrm{MnO}_{3} \quad(x=0,0.5,1)$ and $\mathrm{La}_{0.6} \mathrm{Y}_{0.07} \mathrm{Ca}_{0.33} \mathrm{MnO}_{3}$ pellets between 10 and $300 \mathrm{~K}$. Solid State Communications (2003), Bd. 126(3): S. 147 - 152

[Richert02] RICHERT, Ranko: Heterogeneous dynamics in liquids: fluctuations in space and time. Journal of Physics: Condensed Matter (2002), Bd. 14(23): S. R703

[Riedo03] Riedo, E.; Gnecco, E.; Bennewitz, R.; Meyer, E. und BruNE, H.: Interaction Potential and Hopping Dynamics Governing Sliding Friction. Phys. Rev. Lett. (2003), Bd. 91: S. 084502

[Risken84] Risken, Hannes: Langevin Equations, in: The Fokker-Planck Equation: Methods of Solution and Applications, Springer Berlin Heidelberg, Berlin, Heidelberg (1984), S. 32-62

[Sang01] SAng, Yi; DubÉ, Martin und Grant, Martin: Thermal Effects on Atomic Friction. Phys. Rev. Lett. (2001), Bd. 87: S. 174301

[Santen50] Santen, J.H. Van und JonkeR, G.H.: Electrical conductivity of ferromagnetic compounds of manganese with perovskite structure. Physica (1950), Bd. 16(7): S. 599 - 600 
[Sawatzky17] SAWATzKy, Jörn: Reibung am Phasenübergang einer $\mathrm{VO}_{2}$ Dünnschicht-Probe, Masterarbeit, Georg-August-Universität Göttingen (2017)

[Scherer99] Scherer, V.; Arnold, W. und Bhushan, B.: Lateral force microscopy using acoustic friction force microscopy. Surface and Interface Analysis (1999), Bd. 27(5-6): S. 578-587

[Scherge99] Scherge, M.; Li, X. und Schaefer, J.A.: The effect of water on friction of MEMS. Tribology Letters (1999), Bd. 6(3): S. 215-220

[Schirmeise06] Schirmeisen, André; Jansen, Lars; Hölscher, Hendrik und Fuchs, Harald: Temperature dependence of point contact friction on silicon. Applied Physics Letters (2006), Bd. 88(12): S. 123108

[Schmaltz29] Schmaltz, G: Über Glätte und Ebenheit als physikalisches und physiologisches Problem. Verein Deutscher Ingenieure (1929): S. $1461-1467$

[Schmidt18] ScHMIDT, Hendrik: Nanotribologische Untersuchungen an Dünnschicht-Manganaten: Phononische Beiträge zur Reibung auf der Nanometerskala, Dissertation, Georg-August-Universität Göttingen (2018)

[Schwarzbac16] SchwarzBach, Danny: Magnetismus in $\mathrm{LaMnO}_{3} / \mathrm{SrMnO}_{3}-$ Mehrlagensystemen: Einfluss von Grenzflächen- und Verspannungseffekten, Masterarbeit, Georg-August-Universität Göttingen (2016)

[Shannon76] Shannon, R. D.: Revised effective ionic radii and systematic studies of interatomic distances in halides and chalcogenides. Acta Crystallographica Section A (1976), Bd. 32(5): S. 751-767

[Shibata18] Shibata, Goro; Kitamura, Miho; Minohara, Makoto; Yoshimatsu, Kohei; Kadono, Toshiharu; Ishigami, Keisuke; HARano, Takayuki; Takahashi, Yukio; Sakamoto, Shoya; NoNAKA, Yosuke; IkedA, Keisuke; ChI, Zhendong; Furuse, Mitsuho; Fuchino, Shuichiro; OKano, Makoto; FuJihira, Jun-ichi; 
Uchida, Akira; Watanabe, Kazunori; Fujihira, Hideyuki; FuJihira, Seiichi; Tanaka, Arata; Kumigashira, Hiroshi; KoIDE, Tsuneharu und Fujimori, Atsushi: Anisotropic spin-density distribution and magnetic anisotropy of strained $\mathrm{La}_{1-x} \mathrm{Sr}_{x} \mathrm{MnO}_{3}$ thin films: angle-dependent x-ray magnetic circular dichroism. $n p j$ Quantum Materials (2018), Bd. 3(1): S. 3

[Smith96] Smith, Elizabeth D.; Robbins, Mark O. und Cieplak, Marek: Friction on adsorbed monolayers. Phys. Rev. B (1996), Bd. 54: S. $8252-8260$

[Socoliuc04] Socoliuc, A.; Bennewitz, R.; Gnecco, E. und Meyer, E.: Transition from Stick-Slip to Continuous Sliding in Atomic Friction: Entering a New Regime of Ultralow Friction. Phys. Rev. Lett. (2004), Bd. 92: S. 134301

[Socoliuc06] Socoliuc, Anisoara; Gnecco, Enrico; Maier, Sabine; PfeifFer, Oliver; Baratoff, Alexis; Bennewitz, Roland und MeyER, Ernst: Atomic-Scale Control of Friction by Actuation of Nanometer-Sized Contacts. Science (2006), Bd. 313(5784): S. 207210

[Stillinger95] Stillinger, Frank H.: A Topographic View of Supercooled Liquids and Glass Formation. Science (1995), Bd. 267(5206): S. $1935-1939$

[Stipe01] Stipe, B. C.; Mamin, H. J.; Stowe, T. D.; Kenny, T. W. und Rugar, D.: Noncontact Friction and Force Fluctuations between Closely Spaced Bodies. Phys. Rev. Lett. (2001), Bd. 87: S. 096801

[Sugawara92] Sugawara, Yasuhiro; Fukano, Yoshinobu; Nakano, Akihiko; IDA, Tohru und Morita, Seizo: Oxidation Site of Polycrystalline Silicon Surface Studied Using Scanning Force/Tunneling Microscope (AFM/STM) in Air. Japanese Journal of Applied Physics (1992), Bd. 31(6A): S. L725

[Syono69] Syono, Yasuhiko; iтi Akimoto, Syun und Kohn, Kay: Structure Relations of Hexagonal Perovskite-Like Compounds ABX3 at High Pressure. Journal of the Physical Society of Japan (1969), Bd. 26(4): S. 993-999 
[Tabor57] TABOR, D.: Friction, lubrication and wear of synthetic fibres. Wear (1957), Bd. 1(1): S. $5-24$

[Tabor69] Tabor, D; Winterton, F. R. S. und Winterton, R. H. S.: The direct measurement of normal and retarded van der Waals forces. Proceedings of the Royal Society of London A: Mathematical, Physical and Engineering Sciences (1969), Bd. 312(1511): S. $435-450$

[Takeda74] TakedA, Takayoshi und ŌHArA, Sōji: Magnetic Structure of the Cubic Perovskite Type SrMnO3. Journal of the Physical Society of Japan (1974), Bd. 37(1): S. 275

[Tammann26] Tammann, G. und Hesse, W.: Die Abhängigkeit der Viscosität von der Temperatur bie unterkühlten Flüssigkeiten. Zeitschrift für anorganische und allgemeine Chemie (1926), Bd. 156(1): S. 245257

[Tocha05] Tocha, Ewa; SchönherR, Holger; Julius Vancso, G. und SiEBELT, Natasha: Influence of Grain Size and Humidity on the Nanotribological Properties of Wear-Resistant Nanostructured ZrO2 Coatings: An Atomic Force Microscopy Study. Journal of the American Ceramic Society (2005), Bd. 88(9): S. 2498-2503

[Tokura99] TokurA, Y und TomiokA, Y: Colossal magnetoresistive manganites. Journal of Magnetism and Magnetic Materials (1999), Bd. 200(1): S. $1-23$

[Tomassone97] Tomassone, M. S.; Sokoloff, J. B.; Widom, A. und Krim, J.: Dominance of Phonon Friction for a Xenon Film on a Silver (111) Surface. Phys. Rev. Lett. (1997), Bd. 79: S. 4798-4801

[Tomlinson29] Tombinson, G.A.: CVI. A molecular theory of friction. The London, Edinburgh, and Dublin Philosophical Magazine and Journal of Science (1929), Bd. 7(46): S. 905-939

[Tománek91] Tománek, D.; Zhong, W. und Thomas, H.: Calculation of an Atomically Modulated Friction Force in Atomic-Force Microscopy. EPL (Europhysics Letters) (1991), Bd. 15(8): S. 887 
[Tshiprut09] Tshiprut, Z.; Zelner, S. und Urbakh, M.: TemperatureInduced Enhancement of Nanoscale Friction. Phys. Rev. Lett. (2009), Bd. 102: S. 136102

[Verna10] Verna, A.; Davidson, Bruce A.; Szeto, Y.; Petrov, A.Yu.; Mirone, A.; Giglia, A.; Mahne, N. und Nannarone, S.: Measuring magnetic profiles at manganite surfaces with monolayer resolution. Journal of Magnetism and Magnetic Materials (2010), Bd. 322(9): S. 1212 - 1216, proceedings of the Joint European Magnetic Symposia

[Vogel21] VogeL, Hans: Das Temperaturabhängigkeitsgesetz der Viskosittät von Flüssigkeiten. Phys. Zeit (1921), Bd. 22: S. 645-646

[Vollmer79] Vollmer, H. D. und Risken, H.: Distribution functions for the Brownian motion of particles in a periodic potential driven by an external force. Zeitschrift für Physik B Condensed Matter (1979), Bd. 34(3): S. 313-322

[Volokitin07] Volokitin, A. I.; Persson, B. N. J. und UeBA, H.: Giant enhancement of noncontact friction between closely spaced bodies by dielectric films and two-dimensional systems. Journal of Experimental and Theoretical Physics (2007), Bd. 104(1): S. 96-110

[Wagner11a] WAGNER, Hannes: Lokale Elastizitäsfluktuationen kristalliner und amorpher Festkörper, Diplomarbeit, Georg-August-Universität Göttingen (2011)

[Wagner11b] Wagner, Hannes; Bedorf, Dennis; KüChemann, Stefan; Schwabe, Moritz; Zhang, Bo; Arnold, Walter und Samwer, Konrad: Local elastic properties of a metallic glass. Nature Materials (2011), Bd. 10: S. 439-442

[Watts90] WATts, E. T.; KRIM, J. und Widom, A.: Experimental observation of interfacial slippage at the boundary of molecularly thin films with gold substrates. Phys. Rev. B (1990), Bd. 41: S. 3466-3472

[Weiss96] Weiss, Michael und Elmer, Franz-Josef: Dry friction in the Frenkel-Kontorova-Tomlinson model: Static properties. Phys. Rev. $B$ (1996), Bd. 53: S. 7539-7549 
[Westerburg00] Westerburg, W.; Martin, F.; van Bentum, P.J.M.; PerenBоом, J.A.A.J. und JАков, G.: Charge-carrier density collapse in La0.67Ca0.33MnO3and La0.67Sr0.33MnO3 epitaxial thin films. The European Physical Journal B - Condensed Matter and Complex Systems (2000), Bd. 14(3): S. 509-513

[Yamanaka96] Yamanaka, Kazushi und Nakano, Shizuka: Ultrasonic Atomic Force Microscope with Overtone Excitation of Cantilever. Japanese Journal of Applied Physics (1996), Bd. 35(6S): S. 3787

[Yu13] Yu, Hai-Bin; Wang, Wei-Hua und SAmwer, Konrad: The $\beta$ relaxation in metallic glasses: an overview. Materials Today (2013), Bd. 16(5): S. $183-191$

[Yu14] Yu, Hai Bin; Wang, Wei Hua; Bai, Hai Yang und Samwer, Konrad: The $\beta$-relaxation in metallic glasses. National Science Review (2014), Bd. 1(3): S. 429-461

[Yu15] Yu, Hai-Bin; Richert, Ranko; MaAss, Robert und SAmwer, Konrad: Strain induced fragility transition in metallic glass. $\mathrm{Na}$ ture Communications (2015), Bd. 6: S. 7179

[Yunoki98] Yunoki, S.; Hu, J.; MalvezzI, A. L.; Moreo, A.; Furukawa, N. und Dagotto, E.: Phase Separation in Electronic Models for Manganites. Phys. Rev. Lett. (1998), Bd. 80: S. 845-848

[Yuya08] YuyA, P. A.; Hurley, D. C. und Turner, J. A.: Contactresonance atomic force microscopy for viscoelasticity. Journal of Applied Physics (2008), Bd. 104(7): S. 074916

[Yuya11] Yuya, P. A.; Hurley, D. C. und Turner, J. A.: Relationship between Q-factor and sample damping for contact resonance atomic force microscope measurement of viscoelastic properties. Journal of Applied Physics (2011), Bd. 109(11): S. 113528

[Zhong93] Zhong, Q.; Inniss, D.; KJoller, K. und Elings, V.B.: Fractured polymer/silica fiber surface studied by tapping mode atomic force microscopy. Surface Science (1993), Bd. 290(1): S. L688 L692 


\section{LEBENSLAUF}

In der elektronischen Ausführung dieser Dissertation ist der Lebenslauf des Autors nicht verfügbar. 


\section{VERÖFFENTLICHUNGEN}

01/2017 „Conduction electrons as dissipation channel in friction experiments at the metal-metal transition of LSMO measured by contact-resonance atomic force microscopy"

V. Pfahl, M. K. Phani, M. Büchsenschütz-Göbeler, A. Kumar, V. Moshnyaga, W. Arnold, and K. Samwer, Applied Physics Letters 110, 053102 (2017), [Pfahl17]

01/2018 „Universal Aspects of Sonolubrication in Amorphous and Crystalline Materials"

V. Pfahl, C. Ma, W. Arnold, K. Samwer, Journal of Applied Physics 123, 035301 (2018), [Pfahl18]

07/2018 „Stick-to-Sliding Transition in Contact-Resonance Atomic Force Microscopy"

C. Ma, V. Pfahl, Y. Chen, J. Chu, K. Phani, A. Kumar, W. Arnold, and K. Samwer, Applied Physics Letters (akzeptiert am 31.07.18) ( [Ma18] 


\section{BEITRÄGE AUF KonfEREnZEN}

02/2015 Edgar Lüscher Seminar, Klosters, Schweiz, Kurzvortrag und Poster „Friction in strongly correlated systems under active control“, V. Pfahl, V. Moshnyaga, W. Arnold, C. Volkert, K. Samwer

06/2015 Glastag, Göttingen, Poster

„Friction under active control, here on amorphous PdCuSi“, V. Pfahl, A. Kumar, W. Arnold, C. Volkert, K. Samwer

02/2016 Edgar Lüscher Seminar, Klosters, Schweiz, Kurzvortrag und Poster „Friction under active control“, V. Pfahl, A. Kumar, W. Arnold, C. Volkert, K. Samwer

03/2016 Frühjahrstagung der DPG, Regensburg, Poster „Friction under active control“, V. Pfahl, A. Kumar, W. Arnold, C. Volkert, K. Samwer

02/2017 Edgar Lüscher Seminar, Klosters, Schweiz, Kurzvortrag und Poster „Friction under active control“, V. Pfahl, H. Schmidt, C. Volkert, W. Arnold, K. Samwer

03/2017 Frühjahrstagung der DPG, Dresden, Poster „Friction under active control“, V. Pfahl, H. Schmidt, C. Volkert, W. Arnold, K. Samwer

03/2018 Frühjahrstagung der DPG, Berlin, Poster „Sonolubrication“, V. Pfahl, C. Ma, W. Arnold, K. Samwer 


\section{DANKSAGUNG}

Eine Dissertation schreibt sich nicht von alleine, vor allem aber nicht ohne die Unterstützung vieler Personen. Daher möchte ich zum Abschluss dieser Arbeit diesen Personen danken.

Allen voran gilt mein Dank Prof. Dr. Konrad Samwer, der es mir ermöglichte, meine Promotion am I. Physikalischen Institut durchzuführen. Von seiner intensiven Betreuung, seinem umfangreichen Erfahrungsschatz und seinem weitreichenden Verständnis der Physik konnte ich stets profitieren.

Frau Prof. Dr. Cynthia Volkert danke ich für die Übernahme des Koreferates und für die vielen hilfreichen Diskussionen zum Thema Reibung.

Ein mindestens genauso großer Dank gilt Prof. Dr. Walter Arnold. Ohne die zahlreichen Dialoge, für die er häufig aus dem Saarland angereist ist, seine umfangreichen Literaturkenntnisse und seine technische und physikalische Präzision wäre diese Arbeit in dieser Form nicht möglich gewesen.

Ich danke Prof. Dr. Vasily Moshnyaga für die vielen Gespräche über die Physik der Manganate, seine Interpretationsideen zu neuen Messergebnissen und seine Bereitschaft, meiner Prüfungskommission anzugehören.

Prof. Dr. Stefan Mathias, Dr. Richard Vink und PD.Dr. Martin Wenderoth danke ich ebenso für ihre Zusage, als Prüfungsmitglied an meiner Disputation teilzunehmen.

Ein besonderer Dank gilt allen Mitgliedern und Ehemaligen des I. Physikalischen Instituts, dem ich mich seit Beginn meiner Bachelorarbeitszeit zugehörig fühle, für die entspannte, familiäre und produktive Arbeitsatmosphäre und den ein oder anderen Grilloder Kneipenabend. Insbesondere danke ich Uta Fillipich und Danny Schwarzbach für die Proben, die in dieser Arbeit vermessen wurden, Sebastian Merten für Raman-Messungen und fürs Korrekturlesen, Cinja Seick und Manuel Mchalwat ebenso fürs Korrekturlesen, Matthias Büchsenschütz-Göbeler für die Einführung ins AFM und Carsten Mahn für seine stete Hilfsbereitschaft. Außerdem möchte ich mich herzlich bei meiner Freundin Sina sowie Nils und meinem Vater bedanken, dass sie die Qual, eine fachfremde Arbeit Korrekturlesen zu müssen, auf sich genommen haben.

Meine Familie und meine Freundin haben mich jederzeit und in jeder Hinsicht unterstützt.

Dafür danke ich euch! 